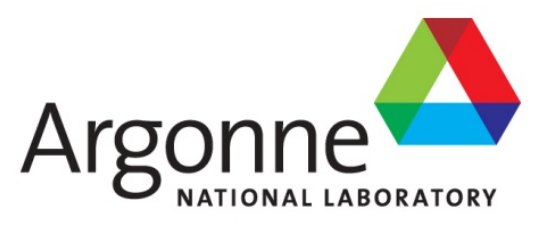

\title{
Derived Concentration Guideline Levels for Argonne National Laboratory's Building 330 Area
}

Environmental Science Division 


\begin{abstract}
About Argonne National Laboratory
Argonne is a U.S. Department of Energy laboratory managed by UChicago Argonne, LLC under contract DE-AC02-06CH11357. The Laboratory's main facility is outside Chicago, at 9700 South Cass Avenue, Argonne, Illinois 60439. For information about Argonne and its pioneering science and technology programs, see www.anl.gov.
\end{abstract}

\title{
Disclaimer
}

This report was prepared as an account of work sponsored by an agency of the United States Government. Neither the United States Government nor any agency thereof, nor UChicago Argonne, LLC, nor any of their employees or officers, makes any warranty, express or implied, or assumes any legal liability or responsibility for the accuracy, completeness, or usefulness of any information, apparatus, product, or process disclosed, or represents that its use would not infringe privately owned rights. Reference herein to any specific commercial product, process, or service by trade name, trademark, manufacturer, or otherwise, does not necessarily constitute or imply its endorsement, recommendation, or favoring by the United States Government or any agency thereof. The views and opinions of document authors expressed herein do not necessarily state or reflect those of the United States Government or any agency thereof, Argonne National Laboratory, or UChicago Argonne, LLC. 


\section{Derived Concentration Guideline Levels for Argonne National Laboratory's Building 330 Area}

prepared by

S. Kamboj and C. Yu

Environmental Science Division, Argonne National Laboratory

March 18, 2011 



\section{CONTENTS}

1 INTRODUCTION

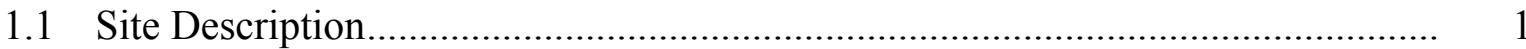

1.2 Building History .....................................................................................

1.3 Derivation of Guideline Levels.................................................................... 1

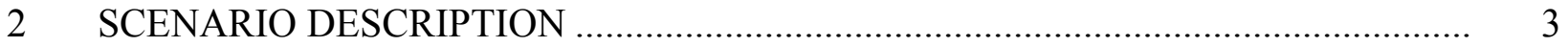

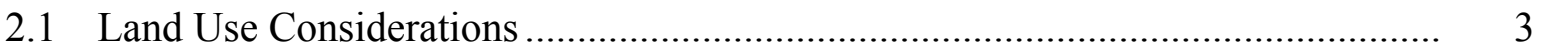

2.2 Exposure Scenarios ............................................................................. 3

2.3 Pathways and Key Parameters ........................................................................ 4

3 DOSE/SOURCE CONCENTRATION RATIOS ................................................. 8

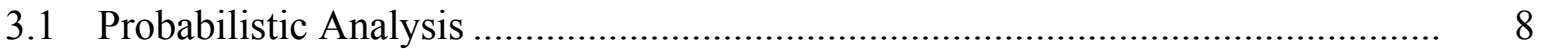

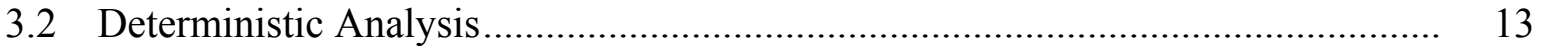

4 DERIVED CONCENTRATION GUIDELINE LEVELS ..................................... 22

4.1 DCGLs Based on Probabilistic Analysis ............................................................ 22

4.2 DCGLs Based on Deterministic Analysis....................................................... 22

4.3 Summary DCGLs for Building 330 Area ..................................................... 22

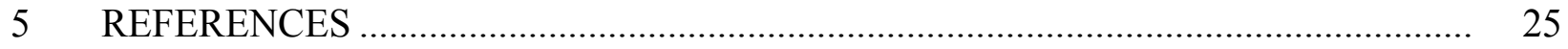

APPENDIX A: Parameters Used for Probabilistic Analysis ........................................... 26

APPENDIX B: $\quad$ Parameters Used for Deterministic Analysis..................................... 51

APPENDIX C: $\quad$ Detailed Probabilistic Analysis Results ........................................... 69

\section{TABLES}

$1 \quad$ Summary of Applicable Exposure Pathways for Different Scenarios Considered for Argonne's Building 330 Area ........................................................... 6

$2 \quad$ List of Key Parameters for Applicable Exposure Pathways …............................... 7

3 Total Peak DSR Percentiles of Am-241 for Different Exposure Scenarios for the Building 330 Area ............................................................................... 10 
TABLES (Cont.)

4 Total Peak DSR Percentiles of Ba-133 for Different Exposure Scenarios for the Building 330 Area

5 Total Peak DSR of C-14 for Different Exposure Scenarios for the Building 330 Area.

6 Total Peak DSR Percentiles of Cs-137 for Different Exposure Scenarios for the Building 330 Area.

7 Total Peak DSR Percentiles of Pu-238 for Different Exposure Scenarios for the Building 330 Area.

8 Total Peak DSR Percentiles of Pu-239 for Different Exposure Scenarios for the Building 330 Area

9 Total Peak DSR Percentiles of Sr-90 for Different Exposure Scenarios for the Building 330 Area

10 Total Peak DSR Percentiles of Tc-99 for Different Exposure Scenarios

for the Building 330 Area.

11 DSRs at Peak of the Means, Mean of the Peaks, and 95th Percentile of the Peaks for Different Scenarios

12 Maximum DSRs for the Industrial Scenario for the Building 330 Area ..................... 19

13 Maximum DSRs for the Offsite Resident Scenario for the Building 330 Area........... 19

14 Maximum DSRs for the Recreational Scenario for the Building 330 Area ................ 20

15 Maximum DSRs for the Onsite Resident Scenario for the Building 330 Area ........... 20

16 Total DSRs for Different Scenarios for the Building 330 Area................................... 21

17 DCGLs Using Probabilistic Analysis at a $25 \mathrm{mrem} / \mathrm{yr}$ Dose Limit for the Building 330 Area .

18 DCGLs Using Deterministic Analysis at the $25 \mathrm{mrem} / \mathrm{yr}$ Dose Limit for Building 330 Area

19 Summary of DCGLs for the Building 330 Area. 


\section{TABLES (Cont.)}

A.1 Parameter Values/Distributions Used in the Analysis for Different Scenarios ............ 27

A.2 Parameter Distributions Used in the Analysis - Contaminated Zone and Unsaturated Zone $K_{d}$ Values........................................................................... 36

A.3 Parameter Distributions Used in the Analysis - Saturated Zone $\mathrm{K}_{\mathrm{d}}$ Values ................ 37

A.4 Parameter Distributions Used in the Analysis for the Plant Transfer Factor................ 38

A.5 Parameter Distribution Used in the Analysis for the Meat Transfer Factor ................. 39

A.6 Parameter Distribution Used in the Analysis for the Milk Transfer Factor.................. 40

A.7 Parameter Value/Distribution Used in the Analysis for the Fish and Crustacea Transfer Factor

A.8 Notations Used in Input Parameter Table 1 ….......................................................... 42

A.9 Parameter Correlations for Probabilistic Analyses in Different Scenarios................... 43

A.10 Additional Input Parameters for RESRAD-OFFSITE Analysis..................................... 44

B.1 Parameter Values Used for Deterministic Analysis of Different Scenarios ................ 52

B.2 Parameter Values Used in the Deterministic Analysis for $\mathrm{K}_{\mathrm{d}}$, Plant, Meat, and Milk Transfer Factors.

B.3 Parameter Value/Distribution Used in the Analysis for the Fish and

Crustacea Transfer Factor

B.4 Additional Input Parameters for RESRAD-OFFSITE Analysis................................... 62

C.1 Peak DSR Percentiles for Different Exposure Pathways for Am-241

in Industrial Use Scenario

C.2 Peak DSR Percentiles for Different Exposure Pathways for Ba-133

in Industrial Use Scenario

C.3 Peak DSR Percentiles for Different Exposure Pathways for C-14 in Industrial Use Scenario

C.4 Peak DSR Percentiles for Different Exposure Pathways for Cs-137 in Industrial Use Scenario 


\section{TABLES (Cont.)}

C.5 Peak DSR Percentiles for Different Exposure Pathways for Pu-238

in Industrial Use Scenario.

C.6 Peak DSR Percentiles for Different Exposure Pathways for Pu-239

in Industrial Use Scenario.

C.7 Peak DSR Percentiles for Different Exposure Pathways for Sr-90

in Industrial Use Scenario

C.8 Peak DSR Percentiles for Different Exposure Pathways for Tc-99

in Industrial Use Scenario.

C.9 Peak DSR Percentiles for Different Exposure Pathways for Am-241

in Offsite Resident Scenario

C.10 Peak DSR Percentiles for Different Exposure Pathways for Ba-133

in Offsite Resident Scenario

C.11 Peak DSR Percentiles for Different Exposure Pathways for C-14

in Offsite Resident Scenario

C.12 Peak DSR Percentiles for Different Exposure Pathways for Cs-137

in Offsite Resident Scenario

C.13 Peak DSR Percentiles for Different Exposure Pathways for Pu-238

in Offsite Resident Scenario

C.14 Peak DSR Percentiles for Different Exposure Pathways for Pu-239

in Offsite Resident Scenario

C.15 Peak DSR Percentiles or Different Exposure Pathways for Sr-90 in

Offsite Resident Scenario

C.16 Peak DSR Percentiles for Different Exposure Pathways for Tc-99

in Offsite Resident Scenario

C.17 Peak DSR Percentiles for Different Exposure Pathways for Am-241 in Recreational Use Scenario

C.18 Peak DSR Percentiles for Different Exposure Pathways for Ba-133

in Recreational Use Scenario 


\section{TABLES (Cont.)}

C.19 Peak DSR Percentiles for Different Exposure Pathways for C-14 in Recreational Use Scenario

C.20 Peak DSR Percentiles for Different Exposure Pathways for Cs-137

in Recreational Use Scenario

C.21 Peak DSR Percentiles for Different Exposure Pathways for Pu-238

in Recreational Use Scenario

C.22 Peak DSR Percentiles for Different Exposure Pathways for Pu-239

in Recreational Use Scenario

C.23 Peak DSR Percentiles for Different Exposure Pathways for Sr-90

in Recreational Use Scenario

C.24 Peak DSR Percentiles for Different Exposure Pathways for Tc-99

in Recreational Use Scenario.

C.25 Peak DSR Percentiles for Different Exposure Pathways for Am-241

in Onsite Resident Scenario

C.26 Peak DSR Percentiles for Different Exposure Pathways for Ba-133

in Onsite Resident Scenario

C.27 Peak DSR Percentiles for Different Exposure Pathways for C-14

in Onsite Resident Scenario

C.28 Peak DSR Percentiles for Different Exposure Pathways for Cs-137

in Onsite Resident Scenario

C.29 Peak DSR Percentiles for Different Exposure Pathways for Pu-238

in Onsite Resident Scenario

C.30 Peak DSR Percentiles for Different Exposure Pathways for Pu-239

in Onsite Resident Scenario

C.31 Peak DSR Percentiles for Different Exposure Pathways for Sr-90

in Onsite Resident Scenario

C.32 Peak DSR Percentiles for Different Exposure Pathways for Tc-99

in Onsite Resident Scenario 


\section{INTRODUCTION}

The derived concentration guideline level (DCGL) is the allowable residual radionuclide concentration that can remain in soil after remediation of the site without radiological restrictions on the use of the site. It is sometimes called the single radionuclide soil guideline or the soil cleanup criteria. This report documents the methodology, scenarios, and parameters used in the analysis to support establishing radionuclide DCGLs for Argonne National Laboratory's Building 330 area.

\subsection{SITE DESCRIPTION}

Argonne National Laboratory (Argonne or ANL) is located $43 \mathrm{~km}$ southwest of downtown Chicago in DuPage County, Illinois. The site occupies 607 ha and is mostly surrounded by the Waterfall Glen Forest Preserve, which is used mainly as a recreational area. The Argonne site contains a number of small ponds and streams. The primary drainage on the site is through Sawmill Creek that runs through the site and enters the Des Plaines River. Some southernmost parts of the site drain directly into the Des Plaines River.

\subsection{BUILDING HISTORY}

Building 330, the former Chicago Pile 5 (CP-5) Reactor facility, was designed for neutron beam, neutron flux, gamma ray, and biological studies in addition to serving as a reactor training facility. The reactor became operational in 1954 and was shut down in 1979. The decommissioning of the reactor and supporting facilities started in 1991 and was completed by the U.S. Department of Energy (DOE) Office of Environmental Management (EM) in late 2000. A final release status survey under DOE Order 5400.5 was completed in 2000 by Argonne Health Physics (ANL-HP) with confirmatory surveys done by the Oak Ridge Institute for Science and Education (ORISE). The building was released for industrial reuse at that time, but still contained significant quantities of tritium in the foundations and soils under the existing concrete slabs. Building 330 was reclassified as below Hazard Category 3 (i.e., no longer considered a nuclear facility). Since it was shut down, the building remained vacant and had deteriorated over time. It was demolished in 2010, and the building's debris disposed of as lowlevel radioactive waste.

\subsection{DERIVATION OF GUIDELINE LEVELS}

The contaminants of concern indentified for Building 330 are Am-241, Ba-133, C-14, Cs-137, Pu-238, Pu-239, Sr-90, and Tc-99 (Gerbig 2011; Matton 2011). The soil guidelines for these radionuclides are calculated using a dose constraint of $25 \mathrm{mrem} / \mathrm{yr}$ for all scenarios. It is assumed that the current industrial use of the site will continue with an institutional control period of 100 years. All land use scenarios are described in Section 2. The soil guidelines are derived for all scenarios, and the results are presented in Section 4. The final DCGLs will be determined by 
DOE after considering other factors such as cost-benefit analysis, as low as reasonably achievable (ALARA) policy, and the applicability to other portions of Argonne site. 


\section{SCENARIO DESCRIPTION}

\subsection{LAND USE CONSIDERATIONS}

The current land use for the site after it is remediated is industrial use with institutional control. However, after the institutional control period ends, the site could be rezoned for other purposes such as recreational use or residential use, or continued as an industrial use site. In developing DCGLs for the Building 330 area's residual soil radioactivity, four potential exposure scenarios are considered to cover all likely future land uses within a 1,000-year time frame. However, if the peak dose were to occur at a later time, the time frame for the analysis would be increased to 10,000 years. The four scenarios vary with respect to the type of land use and food source consumed. Two scenarios (industrial worker onsite and surface water use offsite) capture the dose to different receptors for the current land use conditions. Another two scenarios (recreational use and subsistence farming) capture the potential dose to different receptors after the institutional control period has ended. The industrial use scenario after the institutional control has ended will result in lower doses compared to the current industrial use because the peak dose in the time frame of $1-10,000$ years is used and, due to radioactive decay of some short-lived radionuclides that exist onsite, the dose would be less after the institutional control period of 100 years. Therefore, the industrial scenario after the institutional control period has ended is not considered in this analysis. During the institutional control period, it is possible that contaminants may migrate to offsite locations by either surface runoff or wind erosion. Therefore, offsite residents may be exposed to onsite contamination during and after the institutional control period. This scenario is included in the DCGL analysis.

\subsection{EXPOSURE SCENARIOS}

There are 4 scenarios considered in the derivation of soil guidelines (DCGLs) for the Building 330 area. Two scenarios (Scenario A and B) are current use scenarios and another 2 scenarios (Scenario $C$ and D) are future use scenarios. These 4 scenarios are described in detail in this section. The pathways and parameters considered in these 4 scenarios are discussed in Section 2.3.

Scenario A (the current use onsite worker scenario) assumes continued industrial use of the site. Under this scenario, it is assumed that a receptor would work onsite either indoors or outdoors (a probability distribution representative of an onsite worker will be assigned to the time fraction spent onsite). It is also assumed that the worker will not ingest contaminated water, plant, fish, meat, or milk obtained from the site. It was assumed that the dose to the worker was only from the residual contamination in the soil in the vicinity of Building 330 .

Scenario B (the current use offsite resident scenario) assumes the use of surface water by a hypothetical offsite resident. Under this scenario, it is assumed that the contamination from surface runoff mixes with the storm water drainage and discharges to the Sawmill Creek. A hypothetical offsite resident uses this surface water for irrigation and feeding livestock. However, a public water supply is available in the area, and surface water is not used for 
drinking purposes. The exposure pathways considered are ingestion of plant food grown with the irrigation water from the Sawmill Creek and ingestion of meat and milk from livestock that were fed with water from Sawmill Creek. The fish consumed by the resident are also caught from Sawmill Creek. It should be noted that currently very few people fish in Sawmill Creek or use the water for any purposes. The exposure to external radiation, inhalation of dust and radon, and incidental ingestion of soil are considered for this offsite resident scenario.

Scenario C (the likely future use scenario) assumes recreational use of the site. This scenario assumes that, after the institutional control period, the current forest preserve area bordering the site is expanded to include the Building 330 area. A hypothetical person (recreationist) camps on the remediated area for two weeks per year and is involved in recreational activities such as hiking, skiing, biking, and horseback riding. During this time period, it is assumed that all drinking water used by the hypothetical individual is drawn from the surface water (pond) that captures water from the surface runoff adjacent to the remediated area. Also the individual ingests fish taken from that pond. The site has clayey soil of low permeability and poor drainage; therefore, a pond could be easily built that can capture surface water from runoff. The recreationist does not ingest plant food growing in the area and also does not ingest milk from the livestock or wild animal raised in the area. However, part of the meat diet is from the livestock or wild animal raised in the area.

Scenario D (possible but unlikely future use scenario) assumes residential use of the site after the institutional control period. For this scenario, it is assumed that after the institutional control of the site, the industrial activities would end and the site is re-zoned for residential use. It is assumed that the site is used by a resident farmer. The resident farmer in this situation would be exposed to direct external radiation; internal radiation from inhalation of contaminated dust; internal radiation from inhalation of radon and its progeny; and internal radiation from ingestion of water, plant foods, meat, milk, and fish and incidental ingestion of soil. All water used by the

resident farmer for drinking, irrigation, and household usage is drawn from a deep well adjacent to the remediated area. The individual ingests fish caught from a nearby pond. A shallow well scenario is not considered due to the site hydrogeological characteristics (Patton et al. 1990). Although a perched water table may exist onsite, the water yield of this groundwater system would be low and not capable of sustaining a resident farmer.

\subsection{PATHWAYS AND KEY PARAMETERS}

Potential radiation doses resulting from multiple exposure pathways are considered in this analysis for all exposure scenarios. These pathways include:

1. Direct exposure to external radiation from remediated soil material,

2. Internal radiation from inhalation of contaminated dust,

3. Internal radiation from inhalation of radon and its progeny, 
4. Internal radiation from ingestion of plant foods grown in the remediated area and irrigated with an onsite water source,

5. Internal radiation from ingestion of meat from livestock raised onsite and fed with fodder grown in the remediated area and irrigated with water drawn from an onsite well or pond (the water ingested by livestock is also drawn from an onsite water source),

6. Internal radiation from ingestion of milk from livestock raised onsite and fed with fodder grown in the remediated area and irrigated with onsite well water or pond water (the water ingested by milk cows is also drawn from an onsite water source),

7. Internal radiation from ingestion of fish from a pond located downgradient from the decontaminated area,

8. Internal radiation from incidental ingestion of onsite soil,

9. Internal radiation from drinking water from an onsite water source (groundwater or surface water),

10. Internal radiation from ingestion of plant foods grown offsite and irrigated with contaminated surface water,

11. Internal radiation from ingestion of meat from livestock raised offsite and fed with fodder grown offsite but irrigated with contaminated creek water (the water ingested by livestock is also drawn from the creek),

12. Internal radiation from ingestion of milk from livestock raised offsite and fed with fodder grown offsite but irrigated with contaminated creek water (the water ingested by milk cow is also drawn from the creek), and

13. Ingestion of fish from an offsite surface water source.

Table 1 summarizes the applicable exposure pathways for all scenarios, and Table 2 lists the key parameters for the applicable exposure pathways. 
TABLE 1 Summary of Applicable Exposure Pathways for Different Scenarios Considered for Argonne's Building 330 Area

\begin{tabular}{|c|c|c|c|c|}
\hline \multirow[b]{3}{*}{ Exposure Pathway } & \multicolumn{4}{|c|}{ Applicable Pathways } \\
\hline & \multicolumn{2}{|c|}{ Current Use } & \multicolumn{2}{|c|}{ Future Use } \\
\hline & Scenario $\mathrm{A}^{\mathrm{a}}$ & Scenario $\mathrm{B}^{\mathrm{b}}$ & Scenario $\mathrm{C}^{\mathrm{c}}$ & Scenario $\mathrm{D}^{\mathrm{d}}$ \\
\hline Direct external gamma exposure & Yes & Yes & Yes & Yes \\
\hline Inhalation of dust & Yes & Yes & Yes & Yes \\
\hline Inhalation of radon and its progeny & Yes & Yes & Yes & Yes \\
\hline Ingestion of soil & Yes & Yes & Yes & Yes \\
\hline Ingestion of plant foods grown onsite & No & No & No & Yes \\
\hline Ingestion of meat from livestock raised onsite & No & No & Yes & Yes \\
\hline Ingestion of milk from livestock raised onsite & No & No & No & Yes \\
\hline Ingestion of fish from an onsite pond & No & No & Yes & Yes \\
\hline Ingestion of water from a downgradient well & No & No & No & Yes \\
\hline Ingestion of water from a nearby surface water source & No & No & Yes & No \\
\hline Ingestion of plant foods grown offsite & No & Yes & No & No \\
\hline Ingestion of meat from livestock raised offsite & No & Yes & No & No \\
\hline Ingestion of milk from livestock raised offsite & No & Yes & No & No \\
\hline Ingestion of fish from surface water source offsite & No & Yes & No & No \\
\hline
\end{tabular}

a Industrial worker (current use onsite worker scenario): no consumption of plant food, meat, milk, water, and fish.

b Offsite resident (current use offsite resident scenario): the water used for irrigation and feeding livestock as well as the fish ingested are taken from an offsite surface water body.

c Recreationist (likely future use scenario): no consumption of plant food and milk.

d Onsite resident (possible but unlikely future use scenario): all onsite pathways are included. 
TABLE 2 List of Key Parameters for Applicable Exposure Pathways

Exposure Pathway

Direct external gamma exposure

Inhalation of dust

Inhalation of radon and its progeny

Ingestion of soil

Ingestion of plant foods grown onsite

Ingestion of meat from livestock raised onsite

Ingestion of milk from livestock raised onsite

Ingestion of fish from a nearby pond

Ingestion of water from a

downgradient well

Ingestion of water from a nearby

surface water source

Ingestion of plant foods grown offsite

Ingestion of meat from livestock raised offsite

Ingestion of milk from livestock raised offsite

Ingestion of fish from surface water source offsite
Key Parameters

Time fraction spent onsite and external gamma shielding factor

Inhalation rate, time fraction spent onsite, mass loading for inhalation, and indoor dust filtration factor

Time fraction spent onsite, radon diffusion coefficient and emanation coefficient

Soil ingestion rate and time spent onsite

Plant transfer factor, plant ingestion rate, water dilution factor, and release rate from the source

Plant transfer factor, meat transfer factor, meat ingestion rate, water dilution factor, and release rate from the source

Plant transfer factor, milk transfer factor, milk ingestion rate, water dilution factor, and release rate from the source

Fish bioaccumulation factor, aquatic food contaminated fraction, surface water dilution factor, and release rate from the source

Water ingestion rate, infiltration rate, $K_{d}$ values (or leach rate) for contaminants, and hydrogeological parameters for the site

Water ingestion rate, surface water dilution factor, and release rate from the source

Plant transfer factor, plant ingestion rate, surface water dilution factor, and release rate from the source

Plant transfer factor, meat transfer factor, meat ingestion rate, surface water dilution factor, and release rate from the source

Plant transfer factor, milk transfer factor, milk ingestion rate, surface water dilution factor, and release rate from the source

Fish bioaccumulation factor, aquatic food contaminated fraction, surface water dilution factor, and release rate from the source 


\section{DOSE/SOURCE CONCENTRATION RATIOS}

Each scenario discussed in Section 2 was translated from its potential transport and environmental pathways into a specific set of parameter values. The RESRAD (onsite) and RESRAD-OFFSITE codes include many input parameters that can be classified as metabolic, behavioral, and physical parameters. The metabolic parameter represents a metabolic characteristic of the potential receptor, and its value is independent of the scenario but may be different for different population groups. Behavioral parameter values depend on the receptor's behavior in the scenario (e.g., parameter values for a recreationist scenario could be different from those for a subsistence farmer scenario). The physical parameters are source- and sitespecific, and their values do not change for a different group of receptors. The analysis was done for an adult member of the population. For the behavioral parameters, scenario-specific, or mean, or median values were used. For physical parameters, site-specific values (or distributions), whenever available, were used. If site-specific values were not available, RESRAD (onsite) default values were used. For probabilistic analysis, distributions from the NUREG/CR-6697 report (NRC 2000) were used.

Radionuclide dose/source (concentration) ratios (DSR) were calculated from both probabilistic and deterministic analyses. Deterministic analysis uses a single value for each input parameter, resulting in a single dose output value. The probabilistic analysis uses parameter distributions to identify the variability and uncertainty in dose estimates resulting from variability and uncertainty in the input parameters. A probability distribution is specified for each input parameter with distribution. A model is run repeatedly, using different values for each input parameter with distribution for each run. Instead of giving a single model output as in a deterministic run, probabilistic analysis produces a set of outputs that are equal in number to the number of realizations selected (realizations are equal to the number of observations times number of repetitions).

The RESRAD (onsite) computer code, version 6.5 (Yu et al. 2001), was used for analyzing the industrial, recreational use, and onsite resident scenarios. RESRAD-OFFSITE computer code, version 2.6 (Yu et al. 2007), was used for analyzing the offsite resident scenario. The time frame considered in this analysis was 1,000 years. However, if the peak dose occurred at later time, the time frame for the analysis was increased to 10,000 years. Radioactive decay and ingrowth were considered in deriving dose/source concentration ratios. The various parameters used in the codes for probabilistic analysis are listed in the Appendix A. The various parameters used in the codes for deterministic analysis are listed in the Appendix B.

\subsection{PROBABILISTIC ANALYSIS}

The details of parameter selection are presented in Appendix A, where Table A.1 lists all input parameter distributions/values used in the probabilistic dose analysis for different scenarios. Some of the parameters used in the analysis were radionuclide/element-specific (e.g., $\mathrm{K}_{\mathrm{d}}$ values, transfer factors) and required multiple values because the analysis was 
conducted for multiple radionuclides; therefore, the radionuclide- and element-specific parameters used in the analysis are listed in separate tables.

Parameter distributions for $\mathrm{K}_{\mathrm{d}}$ values are listed in Tables A.2 and A.3. Parameter distributions for plant transfer factors, meat transfer factors, milk transfer factors, and fish and crustacean transfer factors are listed in Tables A.3-A.7, respectively. The distribution function notations used in the RESRAD (onsite) input parameter table (Table A.1) are listed in Table A.8.

Some of the input parameters are correlated. In cases for which a clear relationship exists between parameters, strong correlations were used as input to ensure proper pairing. These include the pairs for bulk density and total porosity (correlation coefficient of -0.99 ), bulk density and effective porosity (correlation coefficient of -0.99), and total porosity and effective porosity (correlation coefficient of 0.99). Table A.9 lists the correlations used in the analysis. A few parameter values and distributions that are scenario-specific and distinguish the scenarios from one another (such as intake rates and time spent onsite) are specified for each scenario. For those parameters not specified with a single value, the default probabilistic distribution was used. This results in a dose probability distribution (presented as the cumulative distribution function, CDF). RESRAD-OFFSITE code requires many more parameters compared to the RESRAD (onsite) code; the additional input parameters for the RESRAD-OFFSITE analysis are listed in Table A.10.

Since the peak dose depends linearly on radionuclide concentration, the analysis was done for unit concentration of each radionuclide for four scenarios. For this analysis, 4,500 input sets (1,500 observations and 3 repetitions) were generated. For each set of sampled parameter values, peak dose in the time interval $0-1,000$ years was calculated. Cumulative probabilities were estimated from the resulting dose distribution for each scenario.

Tables 3-10 provide total peak dose-to-source ratio (DSR) percentiles (mrem/yr per $\mathrm{pCi} / \mathrm{g}$ ) for different exposure scenarios. Also listed in Tables 3-10 are the mean of the peaks and peak of the means DSR. The mean of the peaks DSR is always greater than or equal to the peak of the means DSR. However, if the peak DSRs occurred at the same time for all input sets analyzed, then there would not be any difference in the mean of the peaks and peak of the means DSR. Peak DSR from 4,500 ralizations were analyzed, and the percentiles for different exposure pathways are provided in Appendix C. Water-independent (WI) and water-dependent (WD) pathways are distinguished by the extensions WI and WD, respectively. Tables C.1-C.8 show the peak DSR percentiles for different exposure pathways for individual radionuclides in the industrial use scenario. For Ba-133, Cs-137, and Sr-90, external exposure was the dominant pathway. For Am-241 and Tc-99, external exposure and soil ingestion were the dominant pathways. For $\mathrm{C}-14$, only inhalation was the dominant pathway. For $\mathrm{Pu}-238$ and $\mathrm{Pu}-239$, soil ingestion and inhalation were two dominant pathways.

Tables C.9-C.16 show the peak DSR percentiles for different exposure pathways for individual radionuclides in the offsite resident scenario. For all radionuclides except Ba-133, water-dependent pathways were dominant, and for Ba-133, the water-independent pathway was dominant. For $\mathrm{Am}-241, \mathrm{Pu}-238$, and $\mathrm{Pu}-239$, fish and plant ingestion were dominant pathways. For Ba-133, the external exposure pathway was dominant. For C-14, fish ingestion resulted in 
TABLE 3 Total Peak DSR Percentiles (mrem/yr per pCi/g) of Am-241 for Different Exposure Scenarios for the Building 330 Area

\begin{tabular}{ccccc}
\hline Percentile & Industrial Use & Offsite Resident & Recreational Use & Onsite Resident \\
\hline $5 \%$ & $1.47 \mathrm{E}-03$ & $2.16 \mathrm{E}-03$ & $1.81 \mathrm{E}-03$ & $2.96 \mathrm{E}-02$ \\
$10 \%$ & $4.06 \mathrm{E}-03$ & $2.69 \mathrm{E}-03$ & $1.96 \mathrm{E}-03$ & $3.61 \mathrm{E}-02$ \\
$15 \%$ & $6.76 \mathrm{E}-03$ & $3.32 \mathrm{E}-03$ & $2.05 \mathrm{E}-03$ & $4.05 \mathrm{E}-02$ \\
$20 \%$ & $9.42 \mathrm{E}-03$ & $4.17 \mathrm{E}-03$ & $2.11 \mathrm{E}-03$ & $4.48 \mathrm{E}-02$ \\
$25 \%$ & $1.11 \mathrm{E}-02$ & $4.91 \mathrm{E}-03$ & $2.16 \mathrm{E}-03$ & $4.91 \mathrm{E}-02$ \\
$30 \%$ & $1.21 \mathrm{E}-02$ & $5.62 \mathrm{E}-03$ & $2.22 \mathrm{E}-03$ & $5.33 \mathrm{E}-02$ \\
$35 \%$ & $1.27 \mathrm{E}-02$ & $6.21 \mathrm{E}-03$ & $2.26 \mathrm{E}-03$ & $5.75 \mathrm{E}-02$ \\
$40 \%$ & $1.33 \mathrm{E}-02$ & $6.66 \mathrm{E}-03$ & $2.31 \mathrm{E}-03$ & $6.29 \mathrm{E}-02$ \\
$45 \%$ & $1.37 \mathrm{E}-02$ & $6.92 \mathrm{E}-03$ & $2.36 \mathrm{E}-03$ & $6.81 \mathrm{E}-02$ \\
$50 \%$ & $1.43 \mathrm{E}-02$ & $7.12 \mathrm{E}-03$ & $2.40 \mathrm{E}-03$ & $7.46 \mathrm{E}-02$ \\
$55 \%$ & $1.47 \mathrm{E}-02$ & $7.31 \mathrm{E}-03$ & $2.44 \mathrm{E}-03$ & $8.03 \mathrm{E}-02$ \\
$60 \%$ & $1.52 \mathrm{E}-02$ & $7.49 \mathrm{E}-03$ & $2.49 \mathrm{E}-03$ & $8.78 \mathrm{E}-02$ \\
$65 \%$ & $1.57 \mathrm{E}-02$ & $7.69 \mathrm{E}-03$ & $2.54 \mathrm{E}-03$ & $9.62 \mathrm{E}-02$ \\
$70 \%$ & $1.62 \mathrm{E}-02$ & $7.95 \mathrm{E}-03$ & $2.58 \mathrm{E}-03$ & $1.07 \mathrm{E}-01$ \\
$75 \%$ & $1.68 \mathrm{E}-02$ & $8.26 \mathrm{E}-03$ & $2.64 \mathrm{E}-03$ & $1.20 \mathrm{E}-01$ \\
$80 \%$ & $1.75 \mathrm{E}-02$ & $8.66 \mathrm{E}-03$ & $2.71 \mathrm{E}-03$ & $1.37 \mathrm{E}-01$ \\
$85 \%$ & $1.84 \mathrm{E}-02$ & $9.24 \mathrm{E}-03$ & $2.79 \mathrm{E}-03$ & $1.61 \mathrm{E}-01$ \\
$90 \%$ & $1.97 \mathrm{E}-02$ & $1.00 \mathrm{E}-02$ & $2.89 \mathrm{E}-03$ & $1.96 \mathrm{E}-01$ \\
$95 \%$ & $2.19 \mathrm{E}-02$ & $1.19 \mathrm{E}-02$ & $3.05 \mathrm{E}-03$ & $2.68 \mathrm{E}-01$ \\
Mean of the peaks & $1.35 \mathrm{E}-02$ & $6.98 \mathrm{E}-03$ & $2.40 \mathrm{E}-03$ & $1.05 \mathrm{E}-01$ \\
Peak of the means & $1.35 \mathrm{E}-02$ & $6.87 \mathrm{E}-03$ & $2.40 \mathrm{E}-03$ & $1.01 \mathrm{E}-01$ \\
\hline
\end{tabular}

most dose. For Cs-137, fish, meat, and milk ingestion resulted in most dose. For Sr-90, fish, meat, and plant ingestion resulted in most dose. For Tc-99, plant, fish, and milk ingestion resulted in most dose.

Tables C.17-C.24 show the peak DSR percentiles for different exposure pathways for individual radionuclides in the recreational use scenario. For C-14 and Tc-99, water-dependent pathways were dominant and for others (Am-241, Ba-133, Cs-137, Pu-238, Pu-239, and Sr-90), water-independent pathways were dominant. For $\mathrm{Am}-241, \mathrm{Pu}-238$, and $\mathrm{Pu}-239$, meat ingestion, soil ingestion, and inhalation were dominant pathways. For Ba-133, external exposure was dominant. For C-14 and Tc-99, aquatic food ingestion resulted in most dose. For Cs-137, meat ingestion and external exposure resulted in most dose. For Sr-90, meat ingestion resulted in most dose.

Tables C.25-C.32 show the peak DSR percentiles for different exposure pathways for individual radionuclides in the onsite resident scenario. For C-14 and Tc-99, water-dependent pathways were dominant, and for others (Am-241, Ba-133, Cs-137, Pu-238, Pu-239, and Sr-90), water-independent pathways were dominant. For Am-241, plant ingestion, external exposure, and soil ingestion were dominant. For $\mathrm{Pu}-238$ and $\mathrm{Pu}-239$, plant ingestion, soil ingestion, and inhalation were dominant pathways. For Ba-133, external exposure was dominant. For C-14, aquatic food ingestion resulted in most dose. For Cs-137, external exposure and meat ingestion 
TABLE 4 Total Peak DSR Percentiles (mrem/yr per pCi/g) of Ba-133 for Different Exposure Scenarios for the Building 330 Area

\begin{tabular}{ccccc}
\hline Percentile & Industrial Use & Offsite Resident & Recreational Use & Onsite Resident \\
\hline $5 \%$ & $3.96 \mathrm{E}-02$ & $6.66 \mathrm{E}-05$ & $2.31 \mathrm{E}-07$ & $1.95 \mathrm{E}-06$ \\
$10 \%$ & $1.16 \mathrm{E}-01$ & $7.01 \mathrm{E}-05$ & $1.27 \mathrm{E}-05$ & $9.88 \mathrm{E}-05$ \\
$15 \%$ & $1.93 \mathrm{E}-01$ & $7.37 \mathrm{E}-05$ & $4.04 \mathrm{E}-05$ & $2.71 \mathrm{E}-04$ \\
$20 \%$ & $2.69 \mathrm{E}-01$ & $7.64 \mathrm{E}-05$ & $6.11 \mathrm{E}-05$ & $3.68 \mathrm{E}-04$ \\
$25 \%$ & $3.40 \mathrm{E}-01$ & $7.84 \mathrm{E}-05$ & $7.58 \mathrm{E}-05$ & $4.19 \mathrm{E}-04$ \\
$30 \%$ & $3.61 \mathrm{E}-01$ & $8.00 \mathrm{E}-05$ & $8.46 \mathrm{E}-05$ & $4.60 \mathrm{E}-04$ \\
$35 \%$ & $3.74 \mathrm{E}-01$ & $8.12 \mathrm{E}-05$ & $9.04 \mathrm{E}-05$ & $4.92 \mathrm{E}-04$ \\
$40 \%$ & $3.88 \mathrm{E}-01$ & $8.22 \mathrm{E}-05$ & $9.37 \mathrm{E}-05$ & $5.27 \mathrm{E}-04$ \\
$45 \%$ & $4.01 \mathrm{E}-01$ & $8.31 \mathrm{E}-05$ & $9.62 \mathrm{E}-05$ & $5.65 \mathrm{E}-04$ \\
$50 \%$ & $4.14 \mathrm{E}-01$ & $8.41 \mathrm{E}-05$ & $9.77 \mathrm{E}-05$ & $6.05 \mathrm{E}-04$ \\
$55 \%$ & $4.24 \mathrm{E}-01$ & $8.49 \mathrm{E}-05$ & $9.88 \mathrm{E}-05$ & $6.43 \mathrm{E}-04$ \\
$60 \%$ & $4.33 \mathrm{E}-01$ & $8.58 \mathrm{E}-05$ & $9.95 \mathrm{E}-05$ & $6.79 \mathrm{E}-04$ \\
$65 \%$ & $4.41 \mathrm{E}-01$ & $8.68 \mathrm{E}-05$ & $1.00 \mathrm{E}-04$ & $7.21 \mathrm{E}-04$ \\
$70 \%$ & $4.50 \mathrm{E}-01$ & $8.78 \mathrm{E}-05$ & $1.00 \mathrm{E}-04$ & $7.74 \mathrm{E}-04$ \\
$75 \%$ & $4.60 \mathrm{E}-01$ & $8.87 \mathrm{E}-05$ & $1.01 \mathrm{E}-04$ & $8.30 \mathrm{E}-04$ \\
$80 \%$ & $4.84 \mathrm{E}-01$ & $8.99 \mathrm{E}-05$ & $1.01 \mathrm{E}-04$ & $9.02 \mathrm{E}-04$ \\
$85 \%$ & $5.09 \mathrm{E}-01$ & $9.12 \mathrm{E}-05$ & $1.01 \mathrm{E}-04$ & $9.97 \mathrm{E}-04$ \\
$90 \%$ & $5.34 \mathrm{E}-01$ & $9.30 \mathrm{E}-05$ & $1.01 \mathrm{E}-04$ & $1.11 \mathrm{E}-03$ \\
$95 \%$ & $5.70 \mathrm{E}-01$ & $9.59 \mathrm{E}-05$ & $1.01 \mathrm{E}-04$ & $1.31 \mathrm{E}-03$ \\
Mean of the peaks & $3.80 \mathrm{E}-01$ & $8.32 \mathrm{E}-05$ & $8.04 \mathrm{E}-05$ & $6.32 \mathrm{E}-04$ \\
Peak of the means & $3.80 \mathrm{E}-01$ & $8.06 \mathrm{E}-05$ & $8.04 \mathrm{E}-05$ & $6.32 \mathrm{E}-04$ \\
\hline
\end{tabular}

resulted in most dose. For Sr-90, plant and meat ingestion resulted in most dose. For Tc-99, water and plant ingestion resulted in most dose.

Table 3 shows the total peak DSR percentiles of Am-241 for different exposure scenarios considered in deriving DCGLs for the Building 330 area at Argonne. The future onsite resident (after the institutional control period of 100 years) would receive the highest dose.

Table 4 shows the total peak DSR percentiles of Ba-133 for different exposure scenarios considered in deriving DCGLs for the Building 330 area at Argonne. The industrial worker (current use scenario) would receive the highest dose.

Table 5 shows the total peak DSR percentiles of C-14 for different exposure scenarios considered in deriving DCGLs for the Building 330 area at Argonne. The current offsite resident (current use scenario) would receive the highest dose.

Table 6 shows the total peak DSR percentiles of Cs-137 for different exposure scenarios considered in deriving DCGLs for the Building 330 area at Argonne. The industrial worker (current use scenario) would receive the highest dose. 
TABLE 5 Total Peak DSR Percentiles (mrem/yr per pCi/g) of C-14 for Different Exposure Scenarios for the Building 330 Area

\begin{tabular}{ccccc}
\hline Percentile & Industrial Use & Offsite Resident & Recreational Use & Onsite Resident \\
\hline $5 \%$ & $2.22 \mathrm{E}-06$ & $2.48 \mathrm{E}-04$ & $0.00 \mathrm{E}+00$ & $0.00 \mathrm{E}+00$ \\
$10 \%$ & $5.82 \mathrm{E}-06$ & $3.76 \mathrm{E}-04$ & $0.00 \mathrm{E}+00$ & $0.00 \mathrm{E}+00$ \\
$15 \%$ & $1.02 \mathrm{E}-05$ & $4.97 \mathrm{E}-04$ & $0.00 \mathrm{E}+00$ & $0.00 \mathrm{E}+00$ \\
$20 \%$ & $1.34 \mathrm{E}-05$ & $6.40 \mathrm{E}-04$ & $0.00 \mathrm{E}+00$ & $0.00 \mathrm{E}+00$ \\
$25 \%$ & $1.53 \mathrm{E}-05$ & $8.00 \mathrm{E}-04$ & $0.00 \mathrm{E}+00$ & $0.00 \mathrm{E}+00$ \\
$30 \%$ & $1.68 \mathrm{E}-05$ & $9.86 \mathrm{E}-04$ & $0.00 \mathrm{E}+00$ & $0.00 \mathrm{E}+00$ \\
$35 \%$ & $1.79 \mathrm{E}-05$ & $1.21 \mathrm{E}-03$ & $0.00 \mathrm{E}+00$ & $0.00 \mathrm{E}+00$ \\
$40 \%$ & $1.90 \mathrm{E}-05$ & $1.47 \mathrm{E}-03$ & $0.00 \mathrm{E}+00$ & $0.00 \mathrm{E}+00$ \\
$45 \%$ & $2.02 \mathrm{E}-05$ & $1.85 \mathrm{E}-03$ & $0.00 \mathrm{E}+00$ & $0.00 \mathrm{E}+00$ \\
$50 \%$ & $2.13 \mathrm{E}-05$ & $2.36 \mathrm{E}-03$ & $0.00 \mathrm{E}+00$ & $0.00 \mathrm{E}+00$ \\
$55 \%$ & $2.23 \mathrm{E}-05$ & $2.96 \mathrm{E}-03$ & $0.00 \mathrm{E}+00$ & $0.00 \mathrm{E}+00$ \\
$60 \%$ & $2.34 \mathrm{E}-05$ & $3.89 \mathrm{E}-03$ & $0.00 \mathrm{E}+00$ & $0.00 \mathrm{E}+00$ \\
$65 \%$ & $2.45 \mathrm{E}-05$ & $5.35 \mathrm{E}-03$ & $0.00 \mathrm{E}+00$ & $0.00 \mathrm{E}+00$ \\
$70 \%$ & $2.56 \mathrm{E}-05$ & $7.94 \mathrm{E}-03$ & $1.77 \mathrm{E}-17$ & $0.00 \mathrm{E}+00$ \\
$75 \%$ & $2.70 \mathrm{E}-05$ & $1.27 \mathrm{E}-02$ & $4.24 \mathrm{E}-03$ & $0.00 \mathrm{E}+00$ \\
$80 \%$ & $2.87 \mathrm{E}-05$ & $2.31 \mathrm{E}-02$ & $1.55 \mathrm{E}-02$ & $0.00 \mathrm{E}+00$ \\
$85 \%$ & $3.05 \mathrm{E}-05$ & $4.19 \mathrm{E}-02$ & $5.45 \mathrm{E}-02$ & $1.01 \mathrm{E}-03$ \\
$90 \%$ & $3.32 \mathrm{E}-05$ & $8.49 \mathrm{E}-02$ & $1.17 \mathrm{E}-01$ & $3.45 \mathrm{E}-03$ \\
$95 \%$ & $3.73 \mathrm{E}-05$ & $2.27 \mathrm{E}-01$ & $2.50 \mathrm{E}-01$ & $1.05 \mathrm{E}-02$ \\
Mean of the peaks & $2.10 \mathrm{E}-05$ & $5.95 \mathrm{E}-02$ & $4.61 \mathrm{E}-02$ & $3.36 \mathrm{E}-03$ \\
Peak of the means & $2.10 \mathrm{E}-05$ & $1.50 \mathrm{E}-02$ & $1.58 \mathrm{E}-02$ & $3.54 \mathrm{E}-03$ \\
\hline
\end{tabular}

Table 7 shows the total peak DSR percentiles of Pu-238 for different exposure scenarios considered in deriving DCGLs for the Building 330 area at Argonne. The future onsite resident (after the institutional control period of 100 years) would receive the highest dose.

Table 8 shows the total peak DSR percentiles of $\mathrm{Pu}-239$ for different exposure scenarios considered in deriving DCGLs for the Building 330 area at Argonne. The future onsite resident (after the institutional control period of 100 years) would receive the highest dose.

Table 9 shows the total peak DSR percentiles of Sr-90 for different exposure scenarios considered in deriving DCGLs for the Building 330 area at Argonne. The future onsite resident (after the institutional control period of 100 years) would receive the highest dose.

Table 10 shows the total peak DSR percentiles of Tc-99 for different exposure scenarios considered in deriving DCGLs for the Building 330 area at Argonne. The future onsite resident (after the institutional control period of 100 years) would receive the highest dose.

Table 11 shows the peak of the mean, mean of the peak, and 95th percentile peak DSR of each radionuclide for different scenarios. These values were used in deriving DCGL values. The scenario that results in most dose for and individual radionuclide is highlighted. 
TABLE 6 Total Peak DSR Percentiles (mrem/yr per pCi/g) of Cs-137 for Different Exposure Scenarios for the Building 330 Area

\begin{tabular}{ccccc}
\hline Percentile & Industrial Use & Offsite Resident & Recreational Use & Onsite Resident \\
\hline $5 \%$ & & & & \\
$10 \%$ & $7.12 \mathrm{E}-02$ & $2.69 \mathrm{E}-03$ & $1.27 \mathrm{E}-02$ & $7.02 \mathrm{E}-02$ \\
$15 \%$ & $2.09 \mathrm{E}-01$ & $3.10 \mathrm{E}-03$ & $1.33 \mathrm{E}-02$ & $7.93 \mathrm{E}-02$ \\
$20 \%$ & $3.47 \mathrm{E}-01$ & $3.45 \mathrm{E}-03$ & $1.37 \mathrm{E}-02$ & $8.78 \mathrm{E}-02$ \\
$25 \%$ & $4.85 \mathrm{E}-01$ & $3.76 \mathrm{E}-03$ & $1.42 \mathrm{E}-02$ & $9.41 \mathrm{E}-02$ \\
$30 \%$ & $6.22 \mathrm{E}-01$ & $4.08 \mathrm{E}-03$ & $1.46 \mathrm{E}-02$ & $1.00 \mathrm{E}-01$ \\
$35 \%$ & $6.46 \mathrm{E}-01$ & $4.39 \mathrm{E}-03$ & $1.51 \mathrm{E}-02$ & $1.07 \mathrm{E}-01$ \\
$40 \%$ & $6.70 \mathrm{E}-01$ & $4.71 \mathrm{E}-03$ & $1.55 \mathrm{E}-02$ & $1.14 \mathrm{E}-01$ \\
$45 \%$ & $6.93 \mathrm{E}-01$ & $5.05 \mathrm{E}-03$ & $1.60 \mathrm{E}-02$ & $1.21 \mathrm{E}-01$ \\
$50 \%$ & $7.17 \mathrm{E}-01$ & $5.41 \mathrm{E}-03$ & $1.65 \mathrm{E}-02$ & $1.28 \mathrm{E}-01$ \\
$55 \%$ & $7.41 \mathrm{E}-01$ & $5.76 \mathrm{E}-03$ & $1.71 \mathrm{E}-02$ & $1.36 \mathrm{E}-01$ \\
$60 \%$ & $7.56 \mathrm{E}-01$ & $6.13 \mathrm{E}-03$ & $1.77 \mathrm{E}-02$ & $1.43 \mathrm{E}-01$ \\
$65 \%$ & $7.71 \mathrm{E}-01$ & $6.54 \mathrm{E}-03$ & $1.83 \mathrm{E}-02$ & $1.52 \mathrm{E}-01$ \\
$70 \%$ & $7.87 \mathrm{E}-01$ & $7.07 \mathrm{E}-03$ & $1.92 \mathrm{E}-02$ & $1.61 \mathrm{E}-01$ \\
$75 \%$ & $8.02 \mathrm{E}-01$ & $7.62 \mathrm{E}-03$ & $2.00 \mathrm{E}-02$ & $1.71 \mathrm{E}-01$ \\
$80 \%$ & $8.18 \mathrm{E}-01$ & $8.38 \mathrm{E}-03$ & $2.11 \mathrm{E}-02$ & $1.83 \mathrm{E}-01$ \\
$85 \%$ & $8.62 \mathrm{E}-01$ & $9.14 \mathrm{E}-03$ & $2.25 \mathrm{E}-02$ & $1.99 \mathrm{E}-01$ \\
$90 \%$ & $9.06 \mathrm{E}-01$ & $1.03 \mathrm{E}-02$ & $2.45 \mathrm{E}-02$ & $2.23 \mathrm{E}-01$ \\
$95 \%$ & $9.51 \mathrm{E}-01$ & $1.18 \mathrm{E}-02$ & $2.76 \mathrm{E}-02$ & $2.56 \mathrm{E}-01$ \\
Mean of the peaks & $1.01 \mathrm{E}+00$ & $1.49 \mathrm{E}-02$ & $3.49 \mathrm{E}-02$ & $3.17 \mathrm{E}-01$ \\
Peak of the means & $6.78 \mathrm{E}-01$ & $6.87 \mathrm{E}-03$ & $1.95 \mathrm{E}-02$ & $1.59 \mathrm{E}-01$ \\
& $6.78 \mathrm{E}-01$ & $6.88 \mathrm{E}-03$ & $1.95 \mathrm{E}-02$ & $1.59 \mathrm{E}-01$ \\
\hline
\end{tabular}

\subsection{DETERMINISTIC ANALYSIS}

The details of parameter selection for the deterministic analysis are presented in Appendix B, where Table B.1 lists all input parameter values used in the deterministic dose analysis for different scenarios. Some of the parameters used in the analysis were radionuclide/element-specific (e.g., $\mathrm{K}_{\mathrm{d}}$ values, transfer factors) and required multiple values because analysis was conducted for multiple radionuclides. The parameter values used for these radionuclide- and element-specific analyses are listed in separate tables.

Parameter values for $\mathrm{K}_{\mathrm{d}}$, plant transfer factors, meat transfer factors, and milk transfer factors are listed in Tables B.2. Parameter values for fish and crustacean transfer factors are listed in Table B.3. RESRAD-OFFSITE code requires many more parameters compared to RESRAD (onsite) code; the additional input parameters for RESRAD-OFFSITE analysis are listed in Table B.4.

Table 12 lists the calculated maximum dose/source concentration ratios (DSRs) (mrem/yr per $\mathrm{pCi} / \mathrm{g}$ ) for different exposure pathways for the industrial use scenario. RESRAD (onsite) version 6.5 was used in the analysis. For Ag-108m, Ba-133, Co-60, Cs-137, Eu-152, Eu-154, Eu-155, Np-237, Sr-90, U-235, and U-238, external exposure was the dominant exposure pathway. For Am-241 and Pu-241, external exposure, inhalation, and soil ingestion pathways all 
TABLE 7 Total Peak DSR Percentiles (mrem/yr per pCi/g) of Pu-238 for Different Exposure Scenarios for the Building 330 Area

\begin{tabular}{ccccc}
\hline Percentile & Industrial Use & Offsite Resident & Recreational Use & Onsite Resident \\
\hline $5 \%$ & $6.12 \mathrm{E}-04$ & $8.84 \mathrm{E}-04$ & $6.07 \mathrm{E}-04$ & $1.42 \mathrm{E}-02$ \\
$10 \%$ & $1.65 \mathrm{E}-03$ & $1.18 \mathrm{E}-03$ & $6.87 \mathrm{E}-04$ & $1.74 \mathrm{E}-02$ \\
$15 \%$ & $2.51 \mathrm{E}-03$ & $1.37 \mathrm{E}-03$ & $7.47 \mathrm{E}-04$ & $2.00 \mathrm{E}-02$ \\
$20 \%$ & $3.24 \mathrm{E}-03$ & $1.51 \mathrm{E}-03$ & $8.00 \mathrm{E}-04$ & $2.22 \mathrm{E}-02$ \\
$25 \%$ & $3.85 \mathrm{E}-03$ & $1.65 \mathrm{E}-03$ & $8.37 \mathrm{E}-04$ & $2.48 \mathrm{E}-02$ \\
$30 \%$ & $4.36 \mathrm{E}-03$ & $1.75 \mathrm{E}-03$ & $8.72 \mathrm{E}-04$ & $2.75 \mathrm{E}-02$ \\
$35 \%$ & $4.81 \mathrm{E}-03$ & $1.88 \mathrm{E}-03$ & $9.09 \mathrm{E}-04$ & $3.03 \mathrm{E}-02$ \\
$40 \%$ & $5.21 \mathrm{E}-03$ & $2.02 \mathrm{E}-03$ & $9.42 \mathrm{E}-04$ & $3.33 \mathrm{E}-02$ \\
$45 \%$ & $5.56 \mathrm{E}-03$ & $2.15 \mathrm{E}-03$ & $9.83 \mathrm{E}-04$ & $3.65 \mathrm{E}-02$ \\
$50 \%$ & $5.92 \mathrm{E}-03$ & $2.29 \mathrm{E}-03$ & $1.02 \mathrm{E}-03$ & $4.05 \mathrm{E}-02$ \\
$55 \%$ & $6.27 \mathrm{E}-03$ & $2.47 \mathrm{E}-03$ & $1.06 \mathrm{E}-03$ & $4.43 \mathrm{E}-02$ \\
$60 \%$ & $6.65 \mathrm{E}-03$ & $2.65 \mathrm{E}-03$ & $1.11 \mathrm{E}-03$ & $4.90 \mathrm{E}-02$ \\
$65 \%$ & $7.07 \mathrm{E}-03$ & $2.89 \mathrm{E}-03$ & $1.15 \mathrm{E}-03$ & $5.36 \mathrm{E}-02$ \\
$70 \%$ & $7.54 \mathrm{E}-03$ & $3.17 \mathrm{E}-03$ & $1.20 \mathrm{E}-03$ & $5.97 \mathrm{E}-02$ \\
$75 \%$ & $7.97 \mathrm{E}-03$ & $3.49 \mathrm{E}-03$ & $1.25 \mathrm{E}-03$ & $6.82 \mathrm{E}-02$ \\
$80 \%$ & $8.46 \mathrm{E}-03$ & $3.91 \mathrm{E}-03$ & $1.32 \mathrm{E}-03$ & $7.85 \mathrm{E}-02$ \\
$85 \%$ & $9.12 \mathrm{E}-03$ & $4.62 \mathrm{E}-03$ & $1.40 \mathrm{E}-03$ & $9.26 \mathrm{E}-02$ \\
$90 \%$ & $1.00 \mathrm{E}-02$ & $5.69 \mathrm{E}-03$ & $1.49 \mathrm{E}-03$ & $1.13 \mathrm{E}-01$ \\
$95 \%$ & $1.13 \mathrm{E}-02$ & $8.18 \mathrm{E}-03$ & $1.61 \mathrm{E}-03$ & $1.56 \mathrm{E}-01$ \\
Mean of the peaks & $5.98 \mathrm{E}-03$ & $3.13 \mathrm{E}-03$ & $1.06 \mathrm{E}-03$ & $5.71 \mathrm{E}-02$ \\
Peak of the means & $5.98 \mathrm{E}-03$ & $3.12 \mathrm{E}-03$ & $1.06 \mathrm{E}-03$ & $5.71 \mathrm{E}-02$ \\
\hline
\end{tabular}

contributed to the dose. For C-14, only inhalation was the dominant exposure pathway. For I-129 and Tc-99, soil ingestion and external exposure were dominant exposure pathways. For Ni-59 and $\mathrm{Ni}-63$, soil ingestion was the dominant exposure pathway. For $\mathrm{Pu}-238, \mathrm{Pu}-239$, and $\mathrm{Pu}-240$, inhalation and soil ingestion were two dominant exposure pathways. For U-234, radon inhalation at later time contributed significantly to the dose. For most radionuclides, the maximum dose/source ratio would occur immediately following remedial action. For Pu-241 and U-234, the maximum dose/source concentration ratio would occur at a later time due to buildup of progeny. To check if the peak dose would occur after 1,000 years, the time frame for the analysis was extended to 10,000 years. Table 12 also lists the time of maximum DSR for each radionuclide.

Table 13 lists the calculated maximum DSRs for different exposure pathways for the offsite resident scenario. RESRAD-OFFSITE version 2.6 was used for this analysis. For most radionuclides, water-dependent pathways were dominant. For Ba-133, the external exposure pathway was the dominant exposure pathway. For $\mathrm{Am}-241, \mathrm{Pu}-238$, and $\mathrm{Pu}-239$, fish and plant ingestion were dominant exposure pathways. For $\mathrm{C}-14$, aquatic food ingestion resulted in the most dose. For Cs-137, fish, meat, and milk ingestion resulted in the most dose. For Sr-90, fish, meat, milk, and plant ingestion resulted in the most dose. For Tc-99, plant, fish, and milk ingestion resulted in the most dose. To check if the peak dose would occur after 1,000 years, the 
TABLE 8 Total Peak DSR Percentiles (mrem/yr per pCi/g) of Pu-239 for Different Exposure Scenarios for the Building 330 Area

\begin{tabular}{ccccc}
\hline Percentile & Industrial Use & Offsite Resident & Recreational Use & Onsite Resident \\
\hline $5 \%$ & & & & \\
$10 \%$ & $6.73 \mathrm{E}-04$ & $1.04 \mathrm{E}-03$ & $1.46 \mathrm{E}-03$ & $3.39 \mathrm{E}-02$ \\
$15 \%$ & $1.81 \mathrm{E}-03$ & $1.39 \mathrm{E}-03$ & $1.66 \mathrm{E}-03$ & $4.18 \mathrm{E}-02$ \\
$20 \%$ & $2.76 \mathrm{E}-03$ & $1.64 \mathrm{E}-03$ & $1.80 \mathrm{E}-03$ & $4.76 \mathrm{E}-02$ \\
$25 \%$ & $3.57 \mathrm{E}-03$ & $1.82 \mathrm{E}-03$ & $1.92 \mathrm{E}-03$ & $5.33 \mathrm{E}-02$ \\
$30 \%$ & $4.24 \mathrm{E}-03$ & $1.97 \mathrm{E}-03$ & $2.02 \mathrm{E}-03$ & $5.98 \mathrm{E}-02$ \\
$35 \%$ & $4.79 \mathrm{E}-03$ & $2.13 \mathrm{E}-03$ & $2.10 \mathrm{E}-03$ & $6.62 \mathrm{E}-02$ \\
$40 \%$ & $5.27 \mathrm{E}-03$ & $2.29 \mathrm{E}-03$ & $2.19 \mathrm{E}-03$ & $7.26 \mathrm{E}-02$ \\
$45 \%$ & $5.72 \mathrm{E}-03$ & $2.46 \mathrm{E}-03$ & $2.27 \mathrm{E}-03$ & $7.99 \mathrm{E}-02$ \\
$50 \%$ & $6.11 \mathrm{E}-03$ & $2.60 \mathrm{E}-03$ & $2.36 \mathrm{E}-03$ & $8.80 \mathrm{E}-02$ \\
$55 \%$ & $6.51 \mathrm{E}-03$ & $2.77 \mathrm{E}-03$ & $2.45 \mathrm{E}-03$ & $9.69 \mathrm{E}-02$ \\
$60 \%$ & $6.89 \mathrm{E}-03$ & $2.96 \mathrm{E}-03$ & $2.54 \mathrm{E}-03$ & $1.07 \mathrm{E}-01$ \\
$65 \%$ & $7.30 \mathrm{E}-03$ & $3.20 \mathrm{E}-03$ & $2.65 \mathrm{E}-03$ & $1.18 \mathrm{E}-01$ \\
$70 \%$ & $7.76 \mathrm{E}-03$ & $3.44 \mathrm{E}-03$ & $2.75 \mathrm{E}-03$ & $1.30 \mathrm{E}-01$ \\
$75 \%$ & $8.27 \mathrm{E}-03$ & $3.77 \mathrm{E}-03$ & $2.87 \mathrm{E}-03$ & $1.44 \mathrm{E}-01$ \\
$80 \%$ & $8.75 \mathrm{E}-03$ & $4.15 \mathrm{E}-03$ & $3.02 \mathrm{E}-03$ & $1.64 \mathrm{E}-01$ \\
$85 \%$ & $9.28 \mathrm{E}-03$ & $4.70 \mathrm{E}-03$ & $3.18 \mathrm{E}-03$ & $1.88 \mathrm{E}-01$ \\
$90 \%$ & $1.00 \mathrm{E}-02$ & $5.39 \mathrm{E}-03$ & $3.36 \mathrm{E}-03$ & $2.23 \mathrm{E}-01$ \\
$95 \%$ & $1.10 \mathrm{E}-02$ & $6.51 \mathrm{E}-03$ & $3.56 \mathrm{E}-03$ & $2.70 \mathrm{E}-01$ \\
Mean of the peaks & $1.24 \mathrm{E}-02$ & $9.05 \mathrm{E}-03$ & $3.87 \mathrm{E}-03$ & $3.73 \mathrm{E}-01$ \\
Peak of the means & $6.56 \mathrm{E}-03$ & $3.67 \mathrm{E}-03$ & $2.54 \mathrm{E}-03$ & $1.37 \mathrm{E}-01$ \\
& $6.56 \mathrm{E}-03$ & $3.55 \mathrm{E}-03$ & $2.54 \mathrm{E}-03$ & $1.37 \mathrm{E}-01$ \\
\hline
\end{tabular}

time frame for the analysis was extended to 10,000 years. Table 13 also lists the time of maximum DSR for each radionuclide.

Table 14 lists the maximum DSRs for different exposure pathways for the recreational use scenario. RESRAD (onsite) version 6.5 was used in the analysis. The calculations were done after the institutional control period of 100 years. Maximum DSR would occur at 100 years (immediately after the institutional control period) for all radionuclides. For C-14 and Tc-99, the maximum dose is from water-dependent pathways (such as food ingestion irrigated by contaminated water, water ingestion, and fish ingestion). To check if the peak dose would occur after 1,000 years, the time frame for the analysis was extended to 10,000 years. Table 14 also lists the time of maximum DSR for each radionuclide.

Table 15 lists the maximum DSRs for different exposure pathways for the onsite resident scenario. RESRAD (onsite) version 6.5 was used in the analysis. The calculations were done after the institutional control period of 100 years. Maximum DSRs would occur at 100 years (immediately after the institutional control period) for all radionuclides. For C-14 and Tc-99, the maximum dose is from water-dependent pathways (such as ingestion of food irrigated by contaminated water, water ingestion, and fish ingestion). To check if the peak dose would occur after 1,000 years, the time frame for the analysis was extended to 10,000 years. Table 15 also lists the time of maximum DSR for each radionuclide. 
TABLE 9 Total Peak DSR Percentiles (mrem/yr per pCi/g) of Sr-90 for Different Exposure Scenarios for the Building 330 Area

\begin{tabular}{ccccc}
\hline Percentile & Industrial Use & Offsite Resident & Recreational Use & Onsite Resident \\
\hline $5 \%$ & $9.62 \mathrm{E}-04$ & $8.35 \mathrm{E}-04$ & $1.18 \mathrm{E}-04$ & $4.42 \mathrm{E}-03$ \\
$10 \%$ & $2.84 \mathrm{E}-03$ & $9.28 \mathrm{E}-04$ & $9.18 \mathrm{E}-04$ & $2.97 \mathrm{E}-02$ \\
$15 \%$ & $4.65 \mathrm{E}-03$ & $1.01 \mathrm{E}-03$ & $1.64 \mathrm{E}-03$ & $5.30 \mathrm{E}-02$ \\
$20 \%$ & $6.54 \mathrm{E}-03$ & $1.07 \mathrm{E}-03$ & $2.31 \mathrm{E}-03$ & $7.31 \mathrm{E}-02$ \\
$25 \%$ & $8.25 \mathrm{E}-03$ & $1.14 \mathrm{E}-03$ & $2.88 \mathrm{E}-03$ & $9.77 \mathrm{E}-02$ \\
$30 \%$ & $8.72 \mathrm{E}-03$ & $1.22 \mathrm{E}-03$ & $3.51 \mathrm{E}-03$ & $1.18 \mathrm{E}-01$ \\
$35 \%$ & $9.02 \mathrm{E}-03$ & $1.28 \mathrm{E}-03$ & $4.23 \mathrm{E}-03$ & $1.44 \mathrm{E}-01$ \\
$40 \%$ & $9.35 \mathrm{E}-03$ & $1.36 \mathrm{E}-03$ & $4.93 \mathrm{E}-03$ & $1.70 \mathrm{E}-01$ \\
$45 \%$ & $9.66 \mathrm{E}-03$ & $1.43 \mathrm{E}-03$ & $5.69 \mathrm{E}-03$ & $2.04 \mathrm{E}-01$ \\
$50 \%$ & $9.94 \mathrm{E}-03$ & $1.52 \mathrm{E}-03$ & $6.56 \mathrm{E}-03$ & $2.35 \mathrm{E}-01$ \\
$55 \%$ & $1.02 \mathrm{E}-02$ & $1.60 \mathrm{E}-03$ & $7.69 \mathrm{E}-03$ & $2.79 \mathrm{E}-01$ \\
$60 \%$ & $1.04 \mathrm{E}-02$ & $1.70 \mathrm{E}-03$ & $9.24 \mathrm{E}-03$ & $3.21 \mathrm{E}-01$ \\
$65 \%$ & $1.06 \mathrm{E}-02$ & $1.82 \mathrm{E}-03$ & $1.08 \mathrm{E}-02$ & $3.72 \mathrm{E}-01$ \\
$70 \%$ & $1.09 \mathrm{E}-02$ & $1.95 \mathrm{E}-03$ & $1.28 \mathrm{E}-02$ & $4.25 \mathrm{E}-01$ \\
$75 \%$ & $1.12 \mathrm{E}-02$ & $2.13 \mathrm{E}-03$ & $1.54 \mathrm{E}-02$ & $5.03 \mathrm{E}-01$ \\
$80 \%$ & $1.16 \mathrm{E}-02$ & $2.33 \mathrm{E}-03$ & $1.86 \mathrm{E}-02$ & $6.07 \mathrm{E}-01$ \\
$85 \%$ & $1.22 \mathrm{E}-02$ & $2.62 \mathrm{E}-03$ & $2.34 \mathrm{E}-02$ & $7.50 \mathrm{E}-01$ \\
$90 \%$ & $1.29 \mathrm{E}-02$ & $3.09 \mathrm{E}-03$ & $3.09 \mathrm{E}-02$ & $9.71 \mathrm{E}-01$ \\
$95 \%$ & $1.38 \mathrm{E}-02$ & $3.99 \mathrm{E}-03$ & $4.75 \mathrm{E}-02$ & $1.44 \mathrm{E}+00$ \\
Mean of the peaks & $9.15 \mathrm{E}-03$ & $1.86 \mathrm{E}-03$ & $1.35 \mathrm{E}-02$ & $4.17 \mathrm{E}-01$ \\
Peak of the means & $9.15 \mathrm{E}-03$ & $1.70 \mathrm{E}-03$ & $1.35 \mathrm{E}-02$ & $4.17 \mathrm{E}-01$ \\
\hline
\end{tabular}

Table 16 shows the peak DSR of each radionuclide in different scenarios. These values were used in deriving DCGL values. The scenario that results in most dose for individual radionuclide is highlighted. 
TABLE 10 Total Peak DSR Percentiles (mrem/yr per pCi/g) of Tc-99 for Different Exposure Scenarios for the Building 330 Area

\begin{tabular}{ccccc}
\hline Percentile & Industrial Use & Offsite Resident & Recreational Use & Onsite Resident \\
\hline $5 \%$ & $3.44 \mathrm{E}-06$ & $1.03 \mathrm{E}-04$ & $5.78 \mathrm{E}-06$ & $1.60 \mathrm{E}-05$ \\
$10 \%$ & $9.83 \mathrm{E}-06$ & $2.50 \mathrm{E}-04$ & $1.69 \mathrm{E}-05$ & $4.02 \mathrm{E}-05$ \\
$15 \%$ & $1.62 \mathrm{E}-05$ & $3.90 \mathrm{E}-04$ & $3.34 \mathrm{E}-05$ & $8.08 \mathrm{E}-05$ \\
$20 \%$ & $2.27 \mathrm{E}-05$ & $5.63 \mathrm{E}-04$ & $5.84 \mathrm{E}-05$ & $1.58 \mathrm{E}-04$ \\
$25 \%$ & $2.65 \mathrm{E}-05$ & $7.84 \mathrm{E}-04$ & $9.76 \mathrm{E}-05$ & $5.40 \mathrm{E}-04$ \\
$30 \%$ & $2.87 \mathrm{E}-05$ & $1.02 \mathrm{E}-03$ & $1.50 \mathrm{E}-04$ & $1.68 \mathrm{E}-03$ \\
$35 \%$ & $3.04 \mathrm{E}-05$ & $1.29 \mathrm{E}-03$ & $2.16 \mathrm{E}-04$ & $3.40 \mathrm{E}-03$ \\
$40 \%$ & $3.18 \mathrm{E}-05$ & $1.53 \mathrm{E}-03$ & $3.41 \mathrm{E}-04$ & $5.71 \mathrm{E}-03$ \\
$45 \%$ & $3.30 \mathrm{E}-05$ & $1.79 \mathrm{E}-03$ & $4.72 \mathrm{E}-04$ & $8.61 \mathrm{E}-03$ \\
$50 \%$ & $3.41 \mathrm{E}-05$ & $2.03 \mathrm{E}-03$ & $6.48 \mathrm{E}-04$ & $1.29 \mathrm{E}-02$ \\
$55 \%$ & $3.54 \mathrm{E}-05$ & $2.31 \mathrm{E}-03$ & $8.73 \mathrm{E}-04$ & $1.95 \mathrm{E}-02$ \\
$60 \%$ & $3.65 \mathrm{E}-05$ & $2.59 \mathrm{E}-03$ & $1.17 \mathrm{E}-03$ & $2.79 \mathrm{E}-02$ \\
$65 \%$ & $3.77 \mathrm{E}-05$ & $2.86 \mathrm{E}-03$ & $1.50 \mathrm{E}-03$ & $3.98 \mathrm{E}-02$ \\
$70 \%$ & $3.89 \mathrm{E}-05$ & $3.18 \mathrm{E}-03$ & $1.93 \mathrm{E}-03$ & $5.62 \mathrm{E}-02$ \\
$75 \%$ & $4.02 \mathrm{E}-05$ & $3.54 \mathrm{E}-03$ & $2.49 \mathrm{E}-03$ & $7.78 \mathrm{E}-02$ \\
$80 \%$ & $4.18 \mathrm{E}-05$ & $4.02 \mathrm{E}-03$ & $3.31 \mathrm{E}-03$ & $1.03 \mathrm{E}-01$ \\
$85 \%$ & $4.38 \mathrm{E}-05$ & $4.62 \mathrm{E}-03$ & $4.36 \mathrm{E}-03$ & $1.36 \mathrm{E}-01$ \\
$90 \%$ & $4.70 \mathrm{E}-05$ & $5.50 \mathrm{E}-03$ & $6.31 \mathrm{E}-03$ & $1.83 \mathrm{E}-01$ \\
$95 \%$ & $5.18 \mathrm{E}-05$ & $7.06 \mathrm{E}-03$ & $1.07 \mathrm{E}-02$ & $2.54 \mathrm{E}-01$ \\
Mean of the peaks & $3.21 \mathrm{E}-05$ & $2.59 \mathrm{E}-03$ & $2.56 \mathrm{E}-03$ & $5.68 \mathrm{E}-02$ \\
Peak of the means & $3.21 \mathrm{E}-05$ & $1.06 \mathrm{E}-03$ & $9.62 \mathrm{E}-04$ & $5.41 \mathrm{E}-02$ \\
\hline
\end{tabular}


TABLE 11 DSRs (mrem/yr per pCi/g) at Peak of the Means, Mean of the Peaks, and 95th Percentile of the Peaks for Different Scenarios

\begin{tabular}{|c|c|c|c|c|c|c|c|c|c|c|c|c|}
\hline \multirow[b]{2}{*}{ Nuclide } & \multicolumn{3}{|c|}{ Industrial Use } & \multicolumn{3}{|c|}{ Offsite Resident } & \multicolumn{3}{|c|}{ Recreational Use } & \multicolumn{3}{|c|}{ Onsite Resident } \\
\hline & $\begin{array}{c}\text { Peak of } \\
\text { the Means }\end{array}$ & $\begin{array}{l}\text { Mean of } \\
\text { the Peaks }\end{array}$ & $\begin{array}{l}95 \text { th } \% \text { of } \\
\text { the Peaks }\end{array}$ & $\begin{array}{c}\text { Peak of } \\
\text { the Means }\end{array}$ & $\begin{array}{l}\text { Mean of } \\
\text { the Peaks }\end{array}$ & $\begin{array}{l}95 \text { th } \% \text { of } \\
\text { the Peaks }\end{array}$ & $\begin{array}{c}\text { Peak of } \\
\text { the Means }\end{array}$ & $\begin{array}{l}\text { Mean of } \\
\text { the Peaks }\end{array}$ & $\begin{array}{l}95 \text { th } \% \text { of } \\
\text { the Peaks }\end{array}$ & $\begin{array}{c}\text { Peak of } \\
\text { the Means }\end{array}$ & $\begin{array}{l}\text { Mean of } \\
\text { the Peaks }\end{array}$ & $\begin{array}{l}95 \text { th } \% \text { of } \\
\text { the Peaks }\end{array}$ \\
\hline Am-241 & $1.35 \mathrm{E}-02$ & $1.35 \mathrm{E}-02$ & 2.19E-02 & $6.87 \mathrm{E}-03$ & $6.98 \mathrm{E}-03$ & $1.19 \mathrm{E}-02$ & $2.40 \mathrm{E}-03$ & $2.40 \mathrm{E}-03$ & $3.05 \mathrm{E}-03$ & $1.05 \mathrm{E}-01$ & $1.01 \mathrm{E}-01$ & $2.68 \mathrm{E}-01$ \\
\hline Вa-133 & $3.80 \mathrm{E}-01$ & $3.80 \mathrm{E}-01$ & $5.70 \mathrm{E}-01$ & $8.06 \mathrm{E}-05$ & 8.32E-05 & $9.59 \mathrm{E}-05$ & $8.04 \mathrm{E}-05$ & $8.04 \mathrm{E}-05$ & $1.01 \mathrm{E}-04$ & $6.32 \mathrm{E}-04$ & $6.32 \mathrm{E}-04$ & $1.31 \mathrm{E}-03$ \\
\hline $\mathrm{C}-14$ & $2.10 \mathrm{E}-05$ & $2.10 \mathrm{E}-05$ & $3.73 \mathrm{E}-05$ & $1.50 \mathrm{E}-02$ & $5.95 \mathrm{E}-02$ & $2.27 \mathrm{E}-01$ & $1.58 \mathrm{E}-02$ & 4.61E-02 & $2.50 \mathrm{E}-01$ & $3.54 \mathrm{E}-03$ & $3.36 \mathrm{E}-03$ & $1.05 \mathrm{E}-02$ \\
\hline Cs-137 & $6.78 \mathrm{E}-01$ & $6.78 \mathrm{E}-01$ & $1.01 \mathrm{E}+00$ & $6.88 \mathrm{E}-03$ & $6.87 \mathrm{E}-03$ & $1.49 \mathrm{E}-02$ & $1.95 \mathrm{E}-02$ & $1.95 \mathrm{E}-02$ & $3.49 \mathrm{E}-02$ & $1.59 \mathrm{E}-01$ & $1.59 \mathrm{E}-01$ & 3.17E-01 \\
\hline $\mathrm{Pu}-238$ & $5.98 \mathrm{E}-03$ & $5.98 \mathrm{E}-03$ & $1.13 \mathrm{E}-02$ & $3.12 \mathrm{E}-03$ & $3.13 \mathrm{E}-03$ & $8.18 \mathrm{E}-03$ & $1.06 \mathrm{E}-03$ & $1.06 \mathrm{E}-03$ & $1.61 \mathrm{E}-03$ & $5.71 \mathrm{E}-02$ & $5.71 \mathrm{E}-02$ & $1.56 \mathrm{E}-01$ \\
\hline $\mathrm{Pu}-239$ & $6.56 \mathrm{E}-03$ & $6.56 \mathrm{E}-03$ & $1.24 \mathrm{E}-02$ & $3.55 \mathrm{E}-03$ & $3.67 \mathrm{E}-03$ & $9.05 \mathrm{E}-03$ & $2.54 \mathrm{E}-03$ & $2.54 \mathrm{E}-03$ & $3.87 \mathrm{E}-03$ & $1.37 \mathrm{E}-01$ & $1.37 \mathrm{E}-01$ & $3.73 \mathrm{E}-01$ \\
\hline Sr-90 & $9.15 \mathrm{E}-03$ & $9.15 \mathrm{E}-03$ & $1.38 \mathrm{E}-02$ & $1.70 \mathrm{E}-03$ & $1.86 \mathrm{E}-03$ & $3.99 \mathrm{E}-03$ & $1.35 \mathrm{E}-02$ & $1.35 \mathrm{E}-02$ & $4.75 \mathrm{E}-02$ & 4.17E-01 & 4.17E-01 & $1.44 \mathrm{E}+00$ \\
\hline Tc-99 & $3.21 \mathrm{E}-05$ & $3.21 \mathrm{E}-05$ & $5.18 \mathrm{E}-05$ & $1.06 \mathrm{E}-03$ & $2.59 \mathrm{E}-03$ & $7.06 \mathrm{E}-03$ & $9.62 \mathrm{E}-04$ & $2.56 \mathrm{E}-03$ & $1.07 \mathrm{E}-02$ & $5.41 \mathrm{E}-02$ & $5.68 \mathrm{E}-02$ & $2.54 \mathrm{E}-01$ \\
\hline
\end{tabular}




\section{TABLE 12 Maximum DSRs for the Industrial (Current Use)}

Scenario for the Building 330 Area

\begin{tabular}{lccccc}
\hline \multicolumn{7}{c}{$\begin{array}{c}\text { Maximum Dose/Source Concentration Ratio } \\
\text { (mrem/yr)/(pCi/g) for the Industrial Use Scenario }\end{array}$} & \\
\cline { 2 - 5 } & & & & & \\
Time of \\
Nuclide & $\begin{array}{c}\text { External } \\
\text { Exposure }\end{array}$ & Inhalation & Radon & $\begin{array}{c}\text { Ingestion } \\
\text { of Soil }\end{array}$ & $\begin{array}{c}\text { Maximum } \\
\text { DSR (yr) }\end{array}$ \\
\hline & & & & & \\
Am-241 & $8.23 \mathrm{E}-03$ & $6.60 \mathrm{E}-03$ & $0.00 \mathrm{E}+00$ & $6.16 \mathrm{E}-03$ & $0.00 \mathrm{E}+00$ \\
$\mathrm{Ba}-133$ & $3.81 \mathrm{E}-01$ & $6.66 \mathrm{E}-07$ & $0.00 \mathrm{E}+00$ & $4.48 \mathrm{E}-05$ & $0.00 \mathrm{E}+00$ \\
$\mathrm{C}-14$ & $3.66 \mathrm{E}-07$ & $1.78 \mathrm{E}-05$ & $0.00 \mathrm{E}+00$ & $2.65 \mathrm{E}-06$ & $0.00 \mathrm{E}+00$ \\
$\mathrm{Cs}-137$ & $6.76 \mathrm{E}-01$ & $2.65 \mathrm{E}-06$ & $0.00 \mathrm{E}+00$ & $3.96 \mathrm{E}-04$ & $0.00 \mathrm{E}+00$ \\
Pu-238 & $2.62 \mathrm{E}-05$ & $7.54 \mathrm{E}-03$ & $2.35 \mathrm{E}-13$ & $7.06 \mathrm{E}-03$ & $0.00 \mathrm{E}+00$ \\
Pu-239 & $5.74 \mathrm{E}-05$ & $8.26 \mathrm{E}-03$ & $0.00 \mathrm{E}+00$ & $7.71 \mathrm{E}-03$ & $0.00 \mathrm{E}+00$ \\
Sr-90 & $8.69 \mathrm{E}-03$ & $1.10 \mathrm{E}-05$ & $0.00 \mathrm{E}+00$ & $9.38 \mathrm{E}-04$ & $0.00 \mathrm{E}+00$ \\
Tc-99 & $2.23 \mathrm{E}-05$ & $8.41 \mathrm{E}-07$ & $0.00 \mathrm{E}+00$ & $1.86 \mathrm{E}-05$ & $0.00 \mathrm{E}+00$ \\
\hline
\end{tabular}

a All values are reported to three significant figures. The maximum dose/source concentration ratio would occur at time zero (immediately following remedial action) for all radionuclides.

TABLE 13 Maximum DSRs for the Offsite Resident (Current Use) Scenario for the Building 330 Area

\begin{tabular}{|c|c|c|c|c|c|c|c|c|c|}
\hline \multirow[b]{2}{*}{ Nuclide } & \multicolumn{8}{|c|}{ Maximum Dose/Source Concentration Ratio ${ }^{\mathrm{a}}(\mathrm{mrem} / \mathrm{yr}) /(\mathrm{pCi} / \mathrm{g})$ for Offsite Resident Scenario } & \multirow[b]{2}{*}{$\begin{array}{l}\text { Time of } \\
\text { Maximum } \\
\text { DSR (yr) }\end{array}$} \\
\hline & $\begin{array}{l}\text { External } \\
\text { Exposure }\end{array}$ & Inhalation & Radon & $\begin{array}{c}\text { Plant } \\
\text { Ingestion }\end{array}$ & $\begin{array}{c}\text { Meat } \\
\text { Ingestion }\end{array}$ & $\begin{array}{c}\text { Milk } \\
\text { Ingestion }\end{array}$ & $\begin{array}{c}\text { Soil } \\
\text { Ingestion }\end{array}$ & $\begin{array}{c}\text { Fish } \\
\text { Ingestion }\end{array}$ & \\
\hline Am-241 & $1.09 \mathrm{E}-06$ & $6.57 \mathrm{E}-06$ & $0.00 \mathrm{E}+00$ & $1.32 \mathrm{E}-03$ & $1.05 \mathrm{E}-05$ & $1.56 \mathrm{E}-06$ & 9.18E-07 & $6.69 \mathrm{E}-03$ & 5.86 \\
\hline $\mathrm{Ba}-133^{\mathrm{b}}$ & $7.85 \mathrm{E}-05$ & $4.26 \mathrm{E}-10$ & $0.00 \mathrm{E}+00$ & $6.92 \mathrm{E}-06$ & 2.19E-07 & $2.03 \mathrm{E}-06$ & $5.58 \mathrm{E}-09$ & $6.62 \mathrm{E}-06$ & 6.84 \\
\hline C-14 & $3.98 \mathrm{E}-10$ & $0.00 \mathrm{E}+00$ & $0.00 \mathrm{E}+00$ & 8.02E-05 & 4.52E-05 & 4.57E-05 & 3.54E-09 & $3.38 \mathrm{E}-02$ & 152 \\
\hline Cs-137 & $2.20 \mathrm{E}-04$ & $2.43 \mathrm{E}-09$ & $0.00 \mathrm{E}+00$ & $8.76 \mathrm{E}-05$ & $4.07 \mathrm{E}-04$ & $4.00 \mathrm{E}-04$ & $3.74 \mathrm{E}-08$ & $4.39 \mathrm{E}-03$ & 3.91 \\
\hline $\mathrm{Pu}-238$ & 2.79E-09 & 7.29E-06 & $1.99 \mathrm{E}-15$ & $1.53 \mathrm{E}-03$ & $2.44 \mathrm{E}-05$ & $9.05 \mathrm{E}-07$ & $8.70 \mathrm{E}-07$ & $1.84 \mathrm{E}-03$ & 4.88 \\
\hline $\mathrm{Pu}-239$ & $1.10 \mathrm{E}-06$ & $7.75 \mathrm{E}-06$ & $0.00 \mathrm{E}+00$ & $1.87 \mathrm{E}-03$ & $3.66 \mathrm{E}-05$ & $1.10 \mathrm{E}-06$ & $1.74 \mathrm{E}-04$ & $1.92 \mathrm{E}-03$ & 1118 \\
\hline Sr-90 & $2.89 \mathrm{E}-06$ & $9.73 \mathrm{E}-09$ & $0.00 \mathrm{E}+00$ & 2.74E-04 & 2.82E-04 & $2.43 \mathrm{E}-04$ & $1.11 \mathrm{E}-07$ & $3.94 \mathrm{E}-04$ & 4.88 \\
\hline Tc-99 & $1.44 \mathrm{E}-07$ & $2.02 \mathrm{E}-14$ & $0.00 \mathrm{E}+00$ & $2.70 \mathrm{E}-03$ & $2.49 \mathrm{E}-05$ & 7.19E-04 & $1.43 \mathrm{E}-07$ & $5.74 \mathrm{E}-04$ & 75.2 \\
\hline
\end{tabular}

a All values are reported to three significant figures.

b For Ba-133, the dose is from both water-independent and water-dependent pathways. 


\section{TABLE 14 Maximum DSRs for the Recreational (Likely Future Land Use) Scenario for the Building 330 Area}

\begin{tabular}{|c|c|c|c|c|c|c|c|c|}
\hline \multirow[b]{2}{*}{ Nuclide } & \multicolumn{7}{|c|}{ Maximum Dose/Source Concentration Ratio ${ }^{\mathrm{a}}(\mathrm{mrem} / \mathrm{yr}) /(\mathrm{pCi} / \mathrm{g})$ for Recreational Use Scenario } & \multirow{2}{*}{$\begin{array}{l}\text { Time of } \\
\text { Maximum } \\
\text { DSR (yr) }\end{array}$} \\
\hline & $\begin{array}{c}\text { External } \\
\text { Exposure }\end{array}$ & Inhalation & Radon & $\begin{array}{c}\text { Water } \\
\text { Ingestion }\end{array}$ & $\begin{array}{c}\text { Meat } \\
\text { Ingestion }\end{array}$ & $\begin{array}{c}\text { Soil } \\
\text { Ingestion }\end{array}$ & $\begin{array}{c}\text { Fish } \\
\text { Ingestion }\end{array}$ & \\
\hline Am-241 & $1.18 \mathrm{E}-03$ & $1.16 \mathrm{E}-03$ & $0.00 \mathrm{E}+00$ & $0.00 \mathrm{E}+00$ & $3.05 \mathrm{E}-04$ & 8.81E-04 & $0.00 \mathrm{E}+00$ & $1.00 \mathrm{E}+02$ \\
\hline Ba-133 & $9.80 \mathrm{E}-05$ & $2.10 \mathrm{E}-10$ & $0.00 \mathrm{E}+00$ & $0.00 \mathrm{E}+00$ & $1.73 \mathrm{E}-08$ & $1.15 \mathrm{E}-08$ & $0.00 \mathrm{E}+00$ & $1.00 \mathrm{E}+02$ \\
\hline$C-14^{b}$ & $0.00 \mathrm{E}+00$ & $0.00 \mathrm{E}+00$ & $0.00 \mathrm{E}+00$ & $4.24 \mathrm{E}-17$ & 7.52E-16 & $0.00 \mathrm{E}+00$ & $6.58 \mathrm{E}-13$ & $1.00 \mathrm{E}+02$ \\
\hline Cs-137 & $1.12 \mathrm{E}-02$ & $5.38 \mathrm{E}-08$ & $0.00 \mathrm{E}+00$ & $0.00 \mathrm{E}+00$ & $2.46 \mathrm{E}-03$ & $6.55 \mathrm{E}-06$ & $0.00 \mathrm{E}+00$ & $1.00 \mathrm{E}+02$ \\
\hline $\mathrm{Pu}-238$ & $2.00 \mathrm{E}-06$ & $7.05 \mathrm{E}-04$ & $1.50 \mathrm{E}-13$ & $0.00 \mathrm{E}+00$ & $3.72 \mathrm{E}-04$ & $5.38 \mathrm{E}-04$ & $0.00 \mathrm{E}+00$ & $1.00 \mathrm{E}+02$ \\
\hline $\mathrm{Pu}-239$ & $9.59 \mathrm{E}-06$ & $1.70 \mathrm{E}-03$ & $0.00 \mathrm{E}+00$ & $0.00 \mathrm{E}+00$ & $8.92 \mathrm{E}-04$ & $1.29 \mathrm{E}-03$ & $0.00 \mathrm{E}+00$ & $1.00 \mathrm{E}+02$ \\
\hline Sr-90 & $1.16 \mathrm{E}-04$ & $1.80 \mathrm{E}-07$ & $0.00 \mathrm{E}+00$ & $0.00 \mathrm{E}+00$ & $5.02 \mathrm{E}-03$ & $1.26 \mathrm{E}-05$ & $0.00 \mathrm{E}+00$ & $1.00 \mathrm{E}+02$ \\
\hline Tc-99b & $2.58 \mathrm{E}-12$ & $1.20 \mathrm{E}-13$ & $0.00 \mathrm{E}+00$ & $3.05 \mathrm{E}-06$ & $5.70 \mathrm{E}-09$ & $2.15 \mathrm{E}-12$ & $1.90 \mathrm{E}-05$ & $1.00 \mathrm{E}+02$ \\
\hline
\end{tabular}

a All values are reported to three significant figures. The maximum dose/source concentration ratio would occur at 100 years (immediately after the institutional control period) for all radionuclides.

b For C-14 and Tc-99, the maximum dose is from water dependent pathways (such as ingestion of food irrigated by contaminated water, water ingestion, and fish ingestion).

TABLE 15 Maximum DSRs for the Onsite Resident (Unlikely Future Land Use) Scenario for the Building 330 Area

\begin{tabular}{|c|c|c|c|c|c|c|c|c|c|c|}
\hline \multirow[b]{2}{*}{ Nuclide } & \multicolumn{9}{|c|}{ Maximum Dose/Source Concentration Ratio ${ }^{\mathrm{a}}(\mathrm{mrem} / \mathrm{yr}) /(\mathrm{pCi} / \mathrm{g})$ for On-site Resident Scenario } & \multirow[b]{2}{*}{$\begin{array}{l}\text { Time of } \\
\text { Maximum } \\
\text { DSR (yr) }\end{array}$} \\
\hline & $\begin{array}{c}\text { External } \\
\text { Exposure }\end{array}$ & Inhalation & Radon & $\begin{array}{c}\text { Water } \\
\text { Ingestion }\end{array}$ & $\begin{array}{c}\text { Plant } \\
\text { Ingestion }\end{array}$ & $\begin{array}{c}\text { Meat } \\
\text { Ingestion }\end{array}$ & $\begin{array}{c}\text { Milk } \\
\text { Ingestion }\end{array}$ & $\begin{array}{c}\text { Soil } \\
\text { Ingestion } \\
\end{array}$ & $\begin{array}{c}\text { Fish } \\
\text { Ingestion }\end{array}$ & \\
\hline Am-241 & $1.72 \mathrm{E}-02$ & $6.46 \mathrm{E}-03$ & $0.00 \mathrm{E}+00$ & $0.00 \mathrm{E}+00$ & $5.47 \mathrm{E}-02$ & 5.63E-04 & $3.21 \mathrm{E}-05$ & $1.76 \mathrm{E}-02$ & $0.00 \mathrm{E}+00$ & $1.00 \mathrm{E}+02$ \\
\hline Ba-133 & $1.43 \mathrm{E}-03$ & $1.17 \mathrm{E}-09$ & $0.00 \mathrm{E}+00$ & $0.00 \mathrm{E}+00$ & $3.57 \mathrm{E}-06$ & 4.35E-08 & $1.46 \mathrm{E}-07$ & $2.30 \mathrm{E}-07$ & $0.00 \mathrm{E}+00$ & $1.00 \mathrm{E}+02$ \\
\hline$C-14^{b}$ & $0.00 \mathrm{E}+00$ & $0.00 \mathrm{E}+00$ & $0.00 \mathrm{E}+00$ & 4.63E-14 & $6.49 \mathrm{E}-15$ & $3.98 \mathrm{E}-15$ & $6.24 \mathrm{E}-15$ & $0.00 \mathrm{E}+00$ & $3.45 \mathrm{E}-13$ & $1.00 \mathrm{E}+02$ \\
\hline Cs-137 & $1.64 \mathrm{E}-01$ & $3.00 \mathrm{E}-07$ & $0.00 \mathrm{E}+00$ & $0.00 \mathrm{E}+00$ & $1.63 \mathrm{E}-02$ & $1.42 \mathrm{E}-02$ & $4.65 \mathrm{E}-03$ & $1.31 \mathrm{E}-04$ & $0.00 \mathrm{E}+00$ & $1.00 \mathrm{E}+02$ \\
\hline $\mathrm{Pu}-238$ & $2.92 \mathrm{E}-05$ & $3.93 \mathrm{E}-03$ & $1.75 \mathrm{E}-07$ & $0.00 \mathrm{E}+00$ & 3.34E-02 & $6.87 \mathrm{E}-04$ & $1.04 \mathrm{E}-05$ & $1.07 \mathrm{E}-02$ & $0.00 \mathrm{E}+00$ & $1.00 \mathrm{E}+02$ \\
\hline $\mathrm{Pu}-239$ & $1.40 \mathrm{E}-04$ & $9.45 \mathrm{E}-03$ & $0.00 \mathrm{E}+00$ & $0.00 \mathrm{E}+00$ & $8.00 \mathrm{E}-02$ & $1.65 \mathrm{E}-03$ & $2.35 \mathrm{E}-05$ & $2.57 \mathrm{E}-02$ & $0.00 \mathrm{E}+00$ & $1.00 \mathrm{E}+02$ \\
\hline Sr-90 & $1.70 \mathrm{E}-03$ & $1.01 \mathrm{E}-06$ & $0.00 \mathrm{E}+00$ & $0.00 \mathrm{E}+00$ & $2.34 \mathrm{E}-01$ & $4.72 \mathrm{E}-02$ & $1.40 \mathrm{E}-02$ & $2.51 \mathrm{E}-04$ & $0.00 \mathrm{E}+00$ & $1.00 \mathrm{E}+02$ \\
\hline Tc- $99^{\mathrm{b}}$ & $3.78 \mathrm{E}-11$ & $6.66 \mathrm{E}-13$ & $0.00 \mathrm{E}+00$ & $3.26 \mathrm{E}-03$ & $2.89 \mathrm{E}-04$ & $2.48 \mathrm{E}-06$ & $6.42 \mathrm{E}-05$ & $4.29 \mathrm{E}-11$ & $8.77 \mathrm{E}-06$ & $1.00 \mathrm{E}+02$ \\
\hline
\end{tabular}

a All values are reported to three significant figures. The maximum dose/source concentration ratio would occur at 100 years (immediately after the institutional control period) for all radionuclides.

b For C-14 and Tc-99, the maximum dose is from water dependent pathways (such as food ingestion irrigated by contaminated water, water ingestion, and fish ingestion). 
TABLE 16 Total DSRs for Different Scenarios for the Building 330 Area

\begin{tabular}{lcccc}
\hline & \multicolumn{4}{c}{$\begin{array}{c}\text { Total Dose/Source Concentration Ratio } \\
\text { (mrem/yr)/(pCi/g) for Different Scenarios }\end{array}$} \\
\cline { 2 - 5 } & $\begin{array}{c}\text { Industrial } \\
\text { Use }\end{array}$ & $\begin{array}{c}\text { Offsite } \\
\text { Resident }\end{array}$ & $\begin{array}{c}\text { Onsite } \\
\text { Resident }^{\mathrm{c}}\end{array}$ & $\begin{array}{c}\text { Recreational } \\
\text { Use }^{\mathrm{d}}\end{array}$ \\
\hline & & & & \\
Nuclide & & & & \\
$\mathrm{Am}-241$ & $2.10 \mathrm{E}-02$ & $8.03 \mathrm{E}-03$ & $9.66 \mathrm{E}-02$ & $3.52 \mathrm{E}-03$ \\
$\mathrm{Ba}-133$ & $3.81 \mathrm{E}-01$ & $9.44 \mathrm{E}-05$ & $1.44 \mathrm{E}-03$ & $9.80 \mathrm{E}-05$ \\
$\mathrm{C}-14$ & $2.08 \mathrm{E}-05$ & $3.40 \mathrm{E}-02$ & $4.08 \mathrm{E}-13$ & $6.59 \mathrm{E}-13$ \\
$\mathrm{Cs}-137$ & $6.77 \mathrm{E}-01$ & $5.51 \mathrm{E}-03$ & $1.99 \mathrm{E}-01$ & $1.37 \mathrm{E}-02$ \\
$\mathrm{Pu}-238$ & $1.46 \mathrm{E}-02$ & $3.41 \mathrm{E}-03$ & $4.88 \mathrm{E}-02$ & $1.62 \mathrm{E}-03$ \\
$\mathrm{Pu}-239$ & $1.60 \mathrm{E}-02$ & $4.02 \mathrm{E}-03$ & $1.17 \mathrm{E}-01$ & $3.89 \mathrm{E}-03$ \\
$\mathrm{Sr}-90$ & $9.63 \mathrm{E}-03$ & $1.20 \mathrm{E}-03$ & $2.97 \mathrm{E}-01$ & $5.14 \mathrm{E}-03$ \\
$\mathrm{Tc}-99$ & $4.17 \mathrm{E}-05$ & $4.02 \mathrm{E}-03$ & $3.63 \mathrm{E}-03$ & $2.21 \mathrm{E}-05$ \\
\hline
\end{tabular}

a All values are reported to three significant figures.

b Current use scenario.

c Future unlikely use scenario.

d Future likely use scenario. 


\section{DERIVED CONCENTRATION GUIDELINE LEVELS}

The derived concentration guideline level (DCGL) is the concentration of residual radioactive material that can remain in the decontaminated area and still allow use of the area without radiological restrictions. Given a dose limit of $\mathrm{H}_{E L}$ for an individual, the derived concentration guideline level (DCGL) for an individual radionuclide (i) can be calculated as

$$
\mathrm{DCGL}_{i}(\mathrm{pCi} / \mathrm{g})=\mathrm{H}_{E L}(\mathrm{mrem} / \mathrm{yr}) / \mathrm{DSR}_{i}(\mathrm{mrem} / \mathrm{yr} \text { per pCi/g) }
$$

where $\mathrm{DSR}_{i}$ is the total dose to source concentration ratio. The total DSRs for probabilistic analysis at peak of the means, mean of the peaks, and the 95th percentile of the peaks are listed in Table 11; for deterministic analysis, they are listed in Table 16 for different scenarios. The dose limit, $\mathrm{H}_{\mathrm{EL}}$, used in calculating DCGLs is $25 \mathrm{mrem} / \mathrm{yr}$.

\subsection{DCGLS BASED ON PROBABILISTIC ANALYSIS}

Table 17 shows the DCGLs derived from different scenarios at mean and 95th percentile of the peak DSRs based on a $25 \mathrm{mrem} / \mathrm{yr}$ dose. For some radionuclides, the current land use scenarios (industrial worker or offsite resident scenarios) resulted in the most restrictive DCGLs, and for others, the future land use (onsite resident) scenario resulted in the most restrictive DCGLs. The most restrictive DCGLs are highlighted in Table 17 and listed in the last column under the heading "Most Restrictive" for peak of the means, mean of the peaks, and 95th percentile of the peak DSRs.

\subsection{DCGLS BASED ON DETERMINISTIC ANALYSIS}

Table 18 shows the DCGLs derived from a deterministic analysis of different scenarios based on a $25 \mathrm{mrem} / \mathrm{yr}$ dose limit. For some radionuclides, the current land use scenarios (industrial worker or offsite resident scenarios) resulted in the most restrictive DCGLs and for others, the future land use (onsite resident) scenario resulted in the most restrictive DCGLs. The most restrictive DCGL is highlighted in Table 18 and listed in the last column under the heading "Most Restrictive."

\subsection{SUMMARY DCGLS FOR BUILDING 330 AREA}

Table 19 compares the DCGLs derived based on probabilistic analysis at peak of the means, mean of the peaks, 95th percentile of the peak DSRs, and the DCGLs derived from deterministic analysis for Building 330 area at Argonne. Also listed in the last column of Table 19 are the proposed DCGLs for the eight radionuclides analyzed. These proposed DCGLs are selected by considering both probabilistic and deterministic analyses results, and they are reported to two (2) significant figures. 


\section{TABLE 17 DCGLs (pCi/g) Using Probabilistic Analysis at a $25 \mathrm{mrem} / \mathrm{yr}$ Dose Limit for the Building 330 Area}

\begin{tabular}{|c|c|c|c|c|c|c|c|c|c|c|c|c|c|c|c|}
\hline \multirow[b]{2}{*}{ Nuclide } & \multicolumn{3}{|c|}{ Industrial Use } & \multicolumn{3}{|c|}{ Offsite Resident } & \multicolumn{3}{|c|}{ Recreational Use } & \multicolumn{3}{|c|}{ Onsite Resident } & \multicolumn{3}{|c|}{ Most Restrictive } \\
\hline & $\begin{array}{c}\text { Peak of } \\
\text { the Means }\end{array}$ & $\begin{array}{c}\text { Mean of } \\
\text { the Peaks }\end{array}$ & $\begin{array}{l}\text { 95th of } \\
\text { the Peaks }\end{array}$ & $\begin{array}{c}\text { Peak of } \\
\text { the Means }\end{array}$ & $\begin{array}{c}\text { Mean of } \\
\text { the Peaks }\end{array}$ & $\begin{array}{c}\text { 95th of } \\
\text { the Peaks }\end{array}$ & $\begin{array}{c}\text { Peak of } \\
\text { the Means }\end{array}$ & $\begin{array}{c}\text { Mean of } \\
\text { the Peaks }\end{array}$ & $\begin{array}{c}\text { 95th of } \\
\text { the Peaks }\end{array}$ & $\begin{array}{c}\text { Peak of } \\
\text { the Means }\end{array}$ & $\begin{array}{l}\text { Mean of } \\
\text { the Peaks }\end{array}$ & $\begin{array}{c}\text { 95th of } \\
\text { the Peaks }\end{array}$ & $\begin{array}{c}\text { Peak of } \\
\text { the Means }\end{array}$ & $\begin{array}{c}\text { Mean of } \\
\text { the Peaks }\end{array}$ & $\begin{array}{l}\text { 95th of } \\
\text { the Peaks }\end{array}$ \\
\hline Am-241 & $1.85 \mathrm{E}+03$ & $1.85 \mathrm{E}+03$ & $1.14 \mathrm{E}+03$ & $3.64 \mathrm{E}+04$ & $3.58 \mathrm{E}+03$ & $2.10 \mathrm{E}+03$ & $1.04 \mathrm{E}+04$ & $1.04 \mathrm{E}+04$ & $8.20 \mathrm{E}+03$ & $2.38 \mathrm{E}+02$ & $2.48 \mathrm{E}+02$ & $9.33 \mathrm{E}+01$ & $2.38 \mathrm{E}+02$ & $2.48 \mathrm{E}+02$ & $9.33 \mathrm{E}+01$ \\
\hline Ва-133 & $6.58 \mathrm{E}+01$ & $6.58 \mathrm{E}+01$ & $4.39 \mathrm{E}+01$ & $3.10 \mathrm{E}+05$ & $3.00 \mathrm{E}+05$ & $2.61 \mathrm{E}+05$ & $3.11 \mathrm{E}+05$ & $3.11 \mathrm{E}+05$ & $2.48 \mathrm{E}+05$ & $3.96 \mathrm{E}+04$ & $3.96 \mathrm{E}+04$ & $1.91 \mathrm{E}+04$ & $6.58 \mathrm{E}+01$ & $6.58 \mathrm{E}+01$ & $4.39 \mathrm{E}+01$ \\
\hline C-14 & $1.19 \mathrm{E}+06$ & $1.19 \mathrm{E}+06$ & $6.70 \mathrm{E}+05$ & $1.67 \mathrm{E}+03$ & $4.20 \mathrm{E}+02$ & $1.10 \mathrm{E}+02$ & $1.58 \mathrm{E}+03$ & $5.42 \mathrm{E}+02$ & $1.00 \mathrm{E}+02$ & $7.06 \mathrm{E}+03$ & $1.05 \mathrm{E}+04$ & $2.38 \mathrm{E}+03$ & $1.58 \mathrm{E}+03$ & $4.20 \mathrm{E}+02$ & $1.00 \mathrm{E}+02$ \\
\hline Cs-137 & $3.69 \mathrm{E}+01$ & $3.69 \mathrm{E}+01$ & $2.48 \mathrm{E}+01$ & $3.63 \mathrm{E}+03$ & $3.64 \mathrm{E}+03$ & $1.68 \mathrm{E}+03$ & $1.28 \mathrm{E}+03$ & $1.28 \mathrm{E}+03$ & $7.16 \mathrm{E}+02$ & $1.57 \mathrm{E}+02$ & $1.57 \mathrm{E}+02$ & $7.89 \mathrm{E}+01$ & $3.69 \mathrm{E}+01$ & $3.69 \mathrm{E}+01$ & $2.48 \mathrm{E}+01$ \\
\hline $\mathrm{Pu}-238$ & $4.18 \mathrm{E}+03$ & $4.18 \mathrm{E}+03$ & $2.21 \mathrm{E}+03$ & $8.01 \mathrm{E}+03$ & $7.99 \mathrm{E}+03$ & $3.06 \mathrm{E}+03$ & $2.36 \mathrm{E}+04$ & $2.36 \mathrm{E}+04$ & $1.55 \mathrm{E}+04$ & $4.38 \mathrm{E}+02$ & $4.38 \mathrm{E}+02$ & $1.60 \mathrm{E}+02$ & $4.38 \mathrm{E}+02$ & $4.38 \mathrm{E}+02$ & $1.60 \mathrm{E}+02$ \\
\hline $\mathrm{Pu}-239$ & $3.81 \mathrm{E}+03$ & $3.81 \mathrm{E}+03$ & $2.02 \mathrm{E}+03$ & $7.04 \mathrm{E}+03$ & $6.81 \mathrm{E}+03$ & $2.76 \mathrm{E}+03$ & $9.84 \mathrm{E}+03$ & $9.84 \mathrm{E}+03$ & $6.46 \mathrm{E}+03$ & $1.82 \mathrm{E}+02$ & $1.82 \mathrm{E}+02$ & $6.70 \mathrm{E}+01$ & $1.82 \mathrm{E}+02$ & $1.82 \mathrm{E}+02$ & $6.70 \mathrm{E}+01$ \\
\hline Sr-90 & $2.73 \mathrm{E}+03$ & $2.73 \mathrm{E}+03$ & $1.81 \mathrm{E}+03$ & $1.47 \mathrm{E}+04$ & $1.34 \mathrm{E}+04$ & $6.27 \mathrm{E}+03$ & $1.85 \mathrm{E}+03$ & $1.85 \mathrm{E}+03$ & $5.26 \mathrm{E}+02$ & $6.00 \mathrm{E}+01$ & $6.00 \mathrm{E}+01$ & $1.74 \mathrm{E}+01$ & $6.00 \mathrm{E}+01$ & $6.00 \mathrm{E}+01$ & $1.74 \mathrm{E}+01$ \\
\hline Tc- 99 & $7.79 \mathrm{E}+05$ & $7.79 \mathrm{E}+05$ & $4.83 \mathrm{E}+05$ & $2.36 \mathrm{E}+04$ & $9.65 \mathrm{E}+03$ & $3.54 \mathrm{E}+03$ & $2.60 \mathrm{E}+04$ & $9.77 \mathrm{E}+03$ & $2.34 \mathrm{E}+03$ & $4.62 \mathrm{E}+02$ & $4.40 \mathrm{E}+02$ & $9.84 \mathrm{E}+01$ & $4.62 \mathrm{E}+02$ & $4.40 \mathrm{E}+02$ & $9.84 \mathrm{E}+01$ \\
\hline
\end{tabular}


TABLE 18 DCGLs Using Deterministic Analysis at the $25 \mathrm{mrem} / \mathrm{yr}$ Dose Limit for Building 330 Area

\begin{tabular}{|c|c|c|c|c|c|}
\hline \multirow[b]{3}{*}{ Nuclide } & \multicolumn{5}{|c|}{$\begin{array}{c}\text { DCGLs (pCi/g) from Deterministic Analysis at the } 25 \mathrm{mrem} / \mathrm{yr} \\
\text { Dose Limit from Different Scenarios }\end{array}$} \\
\hline & \multicolumn{2}{|c|}{ Current use } & \multicolumn{2}{|c|}{ Future Use } & \multirow[b]{2}{*}{ Most Restrictive } \\
\hline & Industrial & Offsite Resident & Onsite Resident & Recreational & \\
\hline Am-241 & $1.19 \mathrm{E}+03$ & $3.11 \mathrm{E}+03$ & $2.59 \mathrm{E}+02$ & $7.10 \mathrm{E}+03$ & $2.59 \mathrm{E}+02$ \\
\hline Ba-133 & $6.56 \mathrm{E}+01$ & $2.65 \mathrm{E}+05$ & $1.74 \mathrm{E}+04$ & $2.55 \mathrm{E}+05$ & $6.56 \mathrm{E}+01$ \\
\hline C-14 & $1.20 \mathrm{E}+06$ & $7.36 \mathrm{E}+02$ & $\mathrm{NA}^{\mathrm{a}}$ & $\mathrm{NA}^{\mathrm{a}}$ & $7.36 \mathrm{E}+02$ \\
\hline Cs-137 & $3.70 \mathrm{E}+01$ & $4.54 \mathrm{E}+03$ & $1.26 \mathrm{E}+02$ & $1.83 \mathrm{E}+03$ & $3.70 \mathrm{E}+01$ \\
\hline $\mathrm{Pu}-238$ & $1.71 \mathrm{E}+03$ & $7.33 \mathrm{E}+03$ & $5.13 \mathrm{E}+02$ & $1.55 \mathrm{E}+04$ & $5.13 \mathrm{E}+02$ \\
\hline $\mathrm{Pu}-239$ & $1.56 \mathrm{E}+03$ & $6.23 \mathrm{E}+03$ & $2.14 \mathrm{E}+02$ & $6.44 \mathrm{E}+03$ & $2.14 \mathrm{E}+02$ \\
\hline Sr-90 & $2.60 \mathrm{E}+03$ & $2.09 \mathrm{E}+04$ & $8.42 \mathrm{E}+01$ & $4.86 \mathrm{E}+03$ & $8.42 \mathrm{E}+01$ \\
\hline Tc-99 & $5.99 E+05$ & $6.22 \mathrm{E}+03$ & $6.89 \mathrm{E}+03$ & $1.13 \mathrm{E}+06$ & $6.22 \mathrm{E}+03$ \\
\hline
\end{tabular}

a DCGLs at specific activity limit.

TABLE 19 Summary of DCGLs for the Building 330 Area

\begin{tabular}{|c|c|c|c|c|c|}
\hline \multirow[b]{2}{*}{ Nuclide } & \multicolumn{3}{|c|}{ Probabilistic DCGLs (pCi/g) } & \multirow[b]{2}{*}{$\begin{array}{c}\text { Deterministic } \\
\text { DCGLs (pCi/g) }\end{array}$} & \multirow[b]{2}{*}{$\begin{array}{c}\text { Proposed }^{\mathrm{a}} \\
\text { DCGLs }(\mathrm{pCi} / \mathrm{g})\end{array}$} \\
\hline & $\begin{array}{c}\text { At 95th } \\
\text { Percentile of the } \\
\text { Peak DSRs }\end{array}$ & $\begin{array}{c}\text { At Mean of the } \\
\text { Peak DSRs }\end{array}$ & $\begin{array}{c}\text { At Peak of the } \\
\text { Mean DSRs }\end{array}$ & & \\
\hline Am-241 & $9.33 \mathrm{E}+01$ & $2.38 \mathrm{E}+02$ & $2.48 \mathrm{E}+02$ & $2.59 \mathrm{E}+02$ & $2.4 \mathrm{E}+02$ \\
\hline Ba-133 & $4.39 \mathrm{E}+01$ & $6.58 \mathrm{E}+01$ & $6.58 \mathrm{E}+01$ & $6.56 \mathrm{E}+01$ & $6.5 \mathrm{E}+01$ \\
\hline C-14 & $1.00 \mathrm{E}+02$ & $4.20 \mathrm{E}+02$ & $5.42 \mathrm{E}+02$ & $7.36 \mathrm{E}+02$ & $5.4 \mathrm{E}+02$ \\
\hline Cs-137 & $2.48 \mathrm{E}+01$ & $3.69 \mathrm{E}+01$ & $3.69 \mathrm{E}+01$ & $3.70 \mathrm{E}+01$ & $3.6 \mathrm{E}+01$ \\
\hline $\mathrm{Pu}-238$ & $1.60 \mathrm{E}+02$ & $4.38 \mathrm{E}+02$ & $4.38 \mathrm{E}+02$ & $5.13 \mathrm{E}+02$ & $4.3 \mathrm{E}+02$ \\
\hline $\mathrm{Pu}-239$ & $6.70 \mathrm{E}+01$ & $1.82 \mathrm{E}+02$ & $1.82 \mathrm{E}+02$ & $2.14 \mathrm{E}+02$ & $1.8 \mathrm{E}+02$ \\
\hline Sr-90 & $1.74 \mathrm{E}+01$ & $6.00 \mathrm{E}+01$ & $6.00 \mathrm{E}+01$ & $8.42 \mathrm{E}+01$ & $6.0 \mathrm{E}+01$ \\
\hline Tc-99 & $9.84 \mathrm{E}+01$ & $4.40 \mathrm{E}+02$ & $4.62 \mathrm{E}+02$ & $6.22 \mathrm{E}+03$ & $4.6 \mathrm{E}+02$ \\
\hline
\end{tabular}

a The proposed DCGLs are rounded to 2 significant figures.

The derived concentration guidelines listed in Table 19 are for a large homogenously contaminated area. When implementing the derived concentration guideline levels for decontamination of a site, the sum of fractions rule applies. That is, the summation of the radionuclide concentrations $\mathrm{S}_{i}$ remaining onsite for radioanuclides $i$ divided by their $\mathrm{DCGL}_{i}$ should not be greater than unity; that is,

$$
\sum_{\mathrm{i}} \mathrm{S}_{\mathrm{i}} / \mathrm{DCGL}_{\mathrm{i}} \leq 1
$$




\section{REFERENCES}

Gerbig, C., 2011, Characterization Sample \& Analysis Plan E Wing Building 330 Argonne National Laboratory, prepared by DeNuke Contracting Services, Inc., Oak Ridge, Tenn., revised March 13.

Matton, P.B., 2011, personal communication between P.B. Matton, Argonne National Laboratory, and C. Yu, Argonne National Laboratory, Subject: "Bldg. 330 Soil Characterization Final Report," March 15.

NRC (U.S. Nuclear Regulatory Commission), 2000, Development of Probabilistic RESRAD 6.0 and RESRAD-BUILD 3.0 Computer Codes, NUREG/CR-6697 and ANL/EAD/TM-98, prepared by C. Yu et al., Argonne National Laboratory, Argonne, Ill., for U.S. Nuclear Regulatory Commission, Washington, DC, Nov.

Patton, T.L., R.H. Pearl, and S.Y. Tsai, 1990, Hydrological conditions at the 800 Area at Argonne National Laboratory, ANL/EAIS/TM-29, Argonne National Laboratory, Argonne, Ill., August.

Yu, C., et al., 2007, User's Manual for RESRAD-OFFSITE Version 2, ANL/EVS/TM/07-1, Argonne National Laboratory, Argonne, Ill.

Yu, C., et al., 2001, User's Manual for RESRAD Version 6, ANL/EAD-4, Argonne National Laboratory, Argonne, Ill. 


\section{APPENDIX A:}

\section{PARAMETERS USED FOR PROBABILISTIC ANALYSIS}

Tables A.1-A.10 list all input parameter distributions or parameter values used in the probabilistic dose analysis for different exposure scenarios. 


\section{TABLE A.1 Parameter Values/Distributions Used in the Analysis for Different Scenarios ${ }^{\text {a }}$}

\begin{tabular}{|c|c|c|c|c|c|}
\hline \multirow[b]{2}{*}{ Input Parameters } & \multicolumn{2}{|c|}{ Current Use Scenarios } & \multicolumn{2}{|c|}{ Future Use Scenarios } & \multirow[b]{2}{*}{ Comments } \\
\hline & Industrial Worker & Offsite Resident ${ }^{\mathrm{b}}$ & Resident Farmer $^{\mathrm{b}}$ & Recreationist $^{\mathrm{c}}$ & \\
\hline \multicolumn{6}{|l|}{ Title } \\
\hline Title & Scenario dependent & Scenario dependent & Scenario dependent & Scenario dependent & Scenario definition \\
\hline Dose factor library & ICRP-60 & ICRP-60 & ICRP-60 & ICRP-60 & $\begin{array}{l}\text { ICRP-60 methodology based DCFs } \\
\text { (external from FGR-12 [Eckerman } \\
\text { 1993] and internal from ICRP-72 } \\
\text { [ICRP 1996]) }\end{array}$ \\
\hline Cut-off half-life (180 d or $30 \mathrm{~d}$ ) & $30 \mathrm{~d}$ & $30 \mathrm{~d}$ & $30 \mathrm{~d}$ & $30 \mathrm{~d}$ & RESRAD default \\
\hline Number of points $(32,64,128,256,512,1,024)$ & 32 & 32 & 32 & 32 & RESRAD default \\
\hline Linear spacing/log spacing & Log spacing & Log spacing & Log spacing & Log spacing & RESRAD default \\
\hline Maximum no. of points for dose & 17 & 17 & 17 & 17 & RESRAD default \\
\hline Maximum no. of points for risk & 1 & 1 & 1 & 1 & $\begin{array}{l}\text { Using a smaller integration point } \\
\text { will shorten the calculation time }\end{array}$ \\
\hline Use line draw character (yes/no) & Yes & Yes & Yes & Yes & RESRAD default \\
\hline Find peak pathway dose (yes/no) & Yes & Yes & Yes & Yes & \\
\hline Save all files after each run (yes/no) & Yes & Yes & Yes & Yes & \\
\hline Time integrated probabilistic risk (yes/no) & No & No & No & No & $\begin{array}{l}\text { Dose-to-source ratio (DSR) is } \\
\text { calculated }\end{array}$ \\
\hline \multicolumn{6}{|l|}{ Calculation Parameters } \\
\hline Basic radiation dose limit (mrem/year) & 25 & 25 & 25 & 25 & Not used in DSR calculation \\
\hline Times for calculation (years) & $\begin{array}{l}1,3,10,30,100 \\
300,1,000\end{array}$ & $\begin{array}{l}1,3,10,30,100 \\
\quad 300,1,000\end{array}$ & $100,300,1,000$ & $100,300,1,000$ & $\begin{array}{l}\text { Up to the time horizon for dose } \\
\text { calculation }\end{array}$ \\
\hline \multicolumn{6}{|l|}{ Source } \\
\hline Nuclide concentration (pCi/g) & 1 & 1 & 1 & 1 & Dose-source ratios calculated \\
\hline \multicolumn{6}{|l|}{ Transport Factors } \\
\hline Distribution coefficient for all zones $\left(\mathrm{cm}^{3} / \mathrm{g}\right)$ & Tables A.2 and A.3 & Tables A.2 and A.3 & Tables A.2 and A.3 & Table A.2 and A.3 & $\begin{array}{l}\text { Distribution from Sheppard and } \\
\text { Thibault (1990) }\end{array}$ \\
\hline Number of unsaturated zones & 1 & 1 & 1 & 1 & RESRAD default \\
\hline Time since placement of material (y) & 0 & 0 & 0 & 0 & RESRAD default \\
\hline Groundwater concentration $(\mathrm{pCi} / \mathrm{L})$ & 0 & 0 & 0 & 0 & RESRAD default \\
\hline Leach rate $(1 / y)$ & 0 & 0 & 0 & 0 & RESRAD default \\
\hline
\end{tabular}


TABLE A.1 (Cont.)

Current Use Scenarios

Future Use Scenarios

Input Parameters

Industrial Worker

Offsite Resident ${ }^{\mathrm{b}}$

Resident Farmer ${ }^{\mathrm{b}}$

Recreationist $^{\mathrm{c}}$

Comments

Solubility limit (mol/L)

Use plant/soil ration (check box)

0

0

0
No

No

No

0

RESRAD default

\section{Transfer Factors}

Plant transfer factor (wet-plant weight

concentration, $\mathrm{pCi} / \mathrm{g} / \mathrm{dry}$ soil weight

concentration, $\mathrm{pCi} / \mathrm{g}$ )

Meat transfer factor (concentration in meat,

$\mathrm{pCi} / \mathrm{g} /$ rate of intake, $\mathrm{pCi} / \mathrm{d})$

Milk transfer factor (concentration in milk,

$\mathrm{pCi} / \mathrm{L} /$ rate of intake, $\mathrm{pCi} / \mathrm{d}$ )

Fish transfer factor (concentration in fish,

$\mathrm{pCi} / \mathrm{kg} /$ concentration in water, $\mathrm{pCi} / \mathrm{L}$ )

$\approx \quad$ Crustacea transfer factor (concentration in crustacea, $\mathrm{pCi} / \mathrm{kg} /$ concentration in water, $\mathrm{pCi} / \mathrm{L}$ )

\section{Contaminated Zone Parameters}

Area of contaminated zone $\left(\mathrm{m}^{2}\right)$

Thickness of contaminated zone $(\mathrm{m})$

Length parallel to aquifer flow (m)

Does the initial contamination penetrate the water table?

$\begin{array}{cc}\text { NR }^{\mathrm{d}} & \text { Table A.4 } \\ \text { NR } & \text { Table A.5 } \\ \text { NR } & \text { Table A.6 } \\ \text { NR } & \text { Table A.7 } \\ \text { NR } & \text { Table A.7 } \\ & \\ & \\ 10,000 & \\ 1 & 10,000 \\ 100 & 1 \\ \text { No } & 100 \\ & \text { No }\end{array}$

Table A.4

Table A.5

Table A.6

Table A.7

Table A.7

10,000
1
100
No

10,000

1

100

No
RESRAD default

Distribution from NUREG/CR-6697 (NRC 2000)

Distribution from NUREG/CR-6697 (NRC 2000)

Distribution from NUREG/CR-6697 (NRC 2000)

Table A.7 Distribution from NUREG/CR-6697

(NRC 2000)

RESRAD default

\section{Cover and Contaminated Zone Hydrological}

Data

Cover depth (m)

Density of cover material $\left(\mathrm{g} / \mathrm{cm}^{3}\right)$

Cover erosion rate $(\mathrm{m} / \mathrm{y})$

NR

0

0

0

NR NR

NR

NR

NR

NR

NR

NR

Density of primary contaminated zone $\left(\mathrm{g} / \mathrm{cm}^{3}\right)$

Kamboj 2011; Matton 2011

Kamboj 2011; Matton 2011

RESRAD default

RESRAD default

(1)
$0.999)$

No cover layer assumed

Not required when cover depth equals zero

Not required when cover depth equals zero

Site-specific soil type (silty clay Golchert et al. 2010) distribution 
TABLE A.1 (Cont.)

Current Use Scenarios

Future Use Scenarios

Input Parameters

Contaminated zone erosion rate $(\mathrm{m} / \mathrm{y})$

Contaminated zone total porosity

Contaminated zone field capacity

Contaminated zone hydraulic conductivity

$(\mathrm{m} / \mathrm{y})$

Contaminated zone $\mathrm{b}$ parameter

Humidity in air $\left(\mathrm{g} / \mathrm{m}^{3}\right)$

Evapotranspiration coefficient
Precipitation rate $(\mathrm{m} / \mathrm{y})$

Irrigation rate $(\mathrm{m} / \mathrm{y})$

Irrigation mode (overhead/ditch)

Runoff coefficient

Watershed area for nearby stream or pond $\left(\mathrm{m}^{2}\right)$ Accuracy for water/soil computation

\section{Saturated Zone Hydrological Data}

Density of saturated zone $\left(\mathrm{g} / \mathrm{cm}^{3}\right)$

Saturated zone effective porosity

Saturated zone total porosity

Saturated zone field capacity

Saturated zone hydraulic conductivity $(\mathrm{m} / \mathrm{y})$

\begin{abstract}
Industrial Worker
\end{abstract}
Offsite Resident $\mathrm{t}^{\mathrm{b}}$

Resident Farmer ${ }^{\mathrm{b}}$

Recreationist ${ }^{c}$

Comments

1.65E-4

$1.65 \mathrm{E}-4^{\mathrm{g}}$
$\operatorname{TN}^{\mathrm{f}}(0.36,0.07$,

0.001, 0.999)

0.2

0.32

$\operatorname{TLN}^{f}(2.29$,

$0.259,0.001,0.999$ )

8

$\mathrm{U}(0.5,0.75)$

$$
4.6
$$

0.9177

0.0

0.2

$1,000,000$

0.001

NR

NR

NR
$\operatorname{TN}^{\mathrm{f}}(0.36,0.07$,
0.001, 0.999)

0.2

0.32

$\operatorname{TLN}^{f}(2.29$,

$0.259,0.001,0.999)$

8

$\mathrm{U}(0.5,0.75)$

\section{NR}

0.9177

0.1

NR

0.2

NR

NR
$1.65 \mathrm{E}-4$

$\operatorname{TN}^{\mathrm{f}}(0.36,0.07$,

$0.001,0.999)$

0.2
0.32

$\operatorname{TLN}^{\mathrm{f}}(2.29$, $0.259,0.001,0.999) \quad 0.259,0.001,0.999)$

8

$\mathrm{U}(0.5,0.75)$

4.6

0.9177

0.1

Overhead

0.2

$1,000,000$

0.001
$1.65 \mathrm{E}-4$

$\operatorname{TN}^{\mathrm{f}}(0.36,0.07$, $0.001,0.999)$

0.2

0.32

$\operatorname{TLN}^{\mathrm{f}}(2.29$, 8

$\mathrm{U}(0.5,0.75)$

4.6

0.9177

0.0

NR

0.2

$1,000,000$

0.001
To match the soil erosion of 1 ton/acre/yr in Illinois

(NRCS 2010) Golchert et al. 2010) distribution RESRAD default

Site-specific (Quinn 2010)

Site-specific soil type (silty clay Golchert et al. 2010) distribution

Used only for $\mathrm{H}-3$

Distribution from NUREG/CR-6697 (NRC 2000)

Site-specific value for Chicago (NCDC 2010) Site-specific value from Golchert et al. $2010^{\mathrm{b}}$ Site-specific (ANL 1996) RESRAD default Site-specific for woodland (Yu et al. 2001, page E-7)

RESRAD default RESRAD default
Site-specific soil type (silty clay -
$\mathrm{TN}(1.52,0.230$, $0.001,0.999)$
$\mathrm{TN}(0.355,0.0906$
$0.001,0.999)$
$\mathrm{TN}(0.425,0.0867$
$0.001,0.999)$
0.2

$\mathrm{TN}(1.52,0.230$,

$0.001,0.999)$

$\mathrm{TN}(0.355,0.0906$,

$0.001,0.999)$

$\mathrm{TN}(0.425,0.0867$,

$0.001,0.999)$
$\mathrm{TN}(1.52,0.230$,

$0.001,0.999)$

$\mathrm{TN}(0.355,0.0906, \quad$ Generic soil type distribution

$0.001,0.999)$

$\begin{array}{llll}\mathrm{NR} & 0.2 & 0.2 & 0.2\end{array}$

0.2 
TABLE A.1 (Cont.)

Current Use Scenarios Input Parameters

Saturated zone b parameter

Saturated zone hydraulic gradient

Water table drop rate $(\mathrm{m} / \mathrm{y})$

Well pump intake depth (m below water table)

Model for water transportation

(nondispersion/mass-balance)

Well pumping rate $\left(\mathrm{m}^{3} / \mathrm{y}\right)$

Unsaturated zone parameters

Thickness of unsaturated zone $(\mathrm{m})$

Density of unsaturated zone $\left(\mathrm{g} / \mathrm{cm}^{3}\right)$

Unsaturated zone effective porosity

Unsaturated zone total porosity

Unsaturated zone field capacity

Unsaturated zone hydraulic conductivity $(\mathrm{m} / \mathrm{y})$

Unsaturated zone $\mathrm{b}$ parameter

\section{Occupancy, Inhalation, and External}

\section{Gamma Parameters}

Inhalation rate $\left(\mathrm{m}^{3} / \mathrm{y}\right)$

Mass loading for inhalation $\left(\mathrm{g} / \mathrm{m}^{3}\right)$

Exposure duration (y)

Indoor dust filtration factor

External gamma shielding factor
Future Use Scenarios

\author{
Resident Farmer $^{\mathrm{b}} \quad$ Recreationist $^{\mathrm{c}}$
}

Comments

Industrial Worker $\quad$ Offsite Resident ${ }^{\mathrm{b}} \quad$ Resident Farmer $^{\mathrm{b}} \quad$ Recreationist $^{\mathrm{c}}$

$\operatorname{BLN}(1.06,0.66,0.5$, 30)

$\mathrm{U}(0.007,0.023)$

0.001

5

Nondispersion

250

13.9

13.9

NR $\quad \operatorname{TN}^{\mathrm{f}}(1.696,0.1855$ $0.001,0.999)$

NR $\quad \operatorname{TN}^{\mathrm{f}}(0.289,0.0735$,

$0.001,0.999)$

$\operatorname{TN}^{\mathrm{f}}(0.36,0.07$,

$0.001,0.999)$

0.2

0.32

$\operatorname{TLN}^{f}(2.29$,

$0.259,0.001,0.999)$ $0.001,0.999)$

$\operatorname{TN}^{\mathrm{f}}(0.289,0.0735$, $0.001,0.999)$

$\operatorname{TN}^{\mathrm{f}}(0.36,0.07$, $0.001,0.999)$<smiles></smiles>

0.32

$\operatorname{TLN}^{\mathrm{f}}(2.29$,

$0.259,0.001,0.999)$

\section{$\operatorname{BLN}(1.06,0.66$}

$$
0.5,30)
$$

$\mathrm{U}(0.007,0.023)$

0.001

5

Nondispersion

250

13.9

$\operatorname{TN}^{\mathrm{f}}\left(1.696,0.1855, \quad \mathrm{TN}^{\mathrm{f}}(1.696,0.1855, \quad\right.$ Site-specific soil type (silty clay)
$0.001,0.999)$ distribution

$\operatorname{TN}^{\mathrm{f}}(0.289,0.0735$, Site-specific soil type (silty clay)

$0.001,0.999) \quad$ distribution

$\operatorname{TN}^{\mathrm{f}}(0.36,0.07, \quad$ Site-specific soil type (silty clay)

$0.001,0.999) \quad$ distribution

RESRAD default

0.32

$\operatorname{TLN}^{\mathrm{f}}(2.29, \quad$ Site-specific soil type (silty clay)

$0.259,0.001,0.999) \quad$ distribution
Site-specific (Quinn 2010)
Externa gamma shielding factor

$\begin{array}{cc}11,400 & 8400 \\ \text { Empirical } & \text { Empirical } \\ 25 & 30 \\ \mathrm{U}(0.15,0.95) & \mathrm{U}(0.15,0.95) \\ \mathrm{NR} & \mathrm{BLN}(-1.3,0.59 \\ & 0.044,1)\end{array}$

8400
Empirical
30
$\mathrm{U}(0.15,0.95)$
$\mathrm{BLN}(-1.3,0.59$
$0.044,1)$

14,000

Empirica

30

$\mathrm{U}(0.15,0.95)$

NR
NRC 2000

Not used in DSR calculations

Distribution from NRC 2000

Distribution from NRC 2000
Scenario-specific 
TABLE A.1 (Cont.)

Current Use Scenarios Input Parameters Industrial Worker Offsite Resi

Indoor time fraction

Outdoor time fraction

Shape of contaminated zone

(circular/noncircular)

\section{Ingestion Pathway Dietary Data}

Fruit, vegetable and grain consumption $(\mathrm{kg} / \mathrm{y})$

Leafy vegetable consumption $(\mathrm{kg} / \mathrm{y})$

Milk consumption (L/y)

$\omega \quad$ Meat and poultry consumption $(\mathrm{kg} / \mathrm{y})$

Fish consumption $(\mathrm{kg} / \mathrm{y})$

Other sea food consumption $(\mathrm{kg} / \mathrm{y})$

Soil ingestion $(\mathrm{g} / \mathrm{y})$

Drinking water intake (L/y)

Drinking water contaminated fraction

Household water contaminated fraction

Livestock water contaminated fraction

Irrigation water contaminated fraction

Aquatic food contaminated fraction

Plant food contaminated fraction

Meat contaminated fraction

Milk contaminated fraction 0/empirical

Empirical/0

Circular

NR

NR

NR

NR

NR

NR

$\mathrm{T}(0,18.3,36.5)$

NR

NR

1

NR

NR

NR

NR

NR

NR

Future Use Scenarios

Resident Farmer ${ }^{\mathrm{b}}$

Recreationist $^{\mathrm{c}}$

Comments

NR

NR

Circular

160

14

92

63

0.9

$\mathrm{T}(0,18.3,36.5)$

510

0

1

1

0.5

$-1$

$-1$

$-1$
0.6833

0.0833

Circular

160

14

92

63

5.4

0.9

$\mathrm{T}(0,18.3,36.5)$

510

1

1

1

1
0.5

0.5
-1

$-1$

$-1$

$-1$
0

Scenario-specific (EPA exposure factor) for U-234 industrial

scenario used empirical (because of radon inhalation)

Scenario-specific (EPA exposure factor)

RESRAD default

Milk contaminated fraction

NR


TABLE A.1 (Cont.)

Current Use Scenarios

Future Use Scenarios

\author{
Input Parameters
}

Industrial Worker

Offsite Resident ${ }^{\mathrm{b}}$

Resident Farmer ${ }^{\mathrm{b}}$

Recreationist ${ }^{c}$

Comments

Ingestion Pathway, Nondietary Data

Livestock fodder intake for meat $(\mathrm{kg} / \mathrm{d})$

Livestock fodder intake for milk (kg/d)

Livestock water intake for meat $(\mathrm{L} / \mathrm{d})$

Livestock water intake for milk (L/d)

Livestock intake of soil for meat $(\mathrm{kg} / \mathrm{d})$

Livestock intake of soil for milk $(\mathrm{kg} / \mathrm{d})$

Mass loading for foliar deposition $\left(\mathrm{g} / \mathrm{m}^{3}\right)$

Depth of soil mixing layer (m)

Depth of roots (m)

Drinking water fraction from groundwater source

Household water fraction from groundwater source

Livestock water fraction from groundwater

source

Irrigation water fraction from groundwater source

\section{Plant Factors}

Wet weight crop yield for non-leafy vegetables $\left(\mathrm{kg} / \mathrm{m}^{2}\right)$

Length of growing season for non-leafy

vegetables (y)

Translocation factor for non-leafy vegetables

Weathering removal constant $(1 / \mathrm{y})$

Wet foliar interception fraction for non-leafy

vegetables

\begin{tabular}{|c|c|c|c|c|}
\hline NR & NR & 68 & 10.6 & $\begin{array}{l}\text { RESRAD default or scenario- } \\
\text { specific }\end{array}$ \\
\hline NR & NR & 55 & NR & RESRAD default \\
\hline NR & 50 & 50 & 6.4 & $\begin{array}{l}\text { RESRAD default or scenario- } \\
\text { specific }\end{array}$ \\
\hline NR & 160 & 160 & NR & RESRAD default \\
\hline NR & NR & 0.5 & 0.5 & RESRAD default \\
\hline NR & NR & 0.5 & NR & RESRAD default \\
\hline NR & NR & 0.0001 & 0.0001 & RESRAD default \\
\hline $\mathrm{T}(0.0,0.15,0.6)$ & $\mathrm{T}(0.0,0.15,0.6)$ & $\mathrm{T}(0.0,0.15,0.6)$ & $\mathrm{T}(0.0,0.15,0.6)$ & Distribution from NRC 2000 \\
\hline NR & $\mathrm{U}(0.3,1.0)$ & $\mathrm{U}(0.3,1.0)$ & $\mathrm{U}(0.3,1.0)$ & $\begin{array}{l}\text { Upper bound equal to depth of } \\
\text { contamination }\end{array}$ \\
\hline NR & 0 & 1 & 0 & Scenario-specific \\
\hline NR & 0 & 1 & NR & Scenario-specific \\
\hline NR & 0 & 1 & 0 & $\begin{array}{l}\text { RESRAD default for resident farmer } \\
\text { scenario and } 0 \text { for recreational use } \\
\text { scenario because surface water is } \\
\text { used by livestock }\end{array}$ \\
\hline NR & 0 & 1 & 0 & RESRAD default \\
\hline NR & $\begin{array}{c}\operatorname{TLN}(0.56,0.48 \\
0.001,0.999)\end{array}$ & $\begin{array}{c}\operatorname{TLN}(0.56,0.48 \\
0.001,0.999)\end{array}$ & NR & Distribution from NRC 2000 \\
\hline NR & 0.17 & 0.17 & NR & RESRAD default \\
\hline NR & 0.1 & 0.1 & NR & RESRAD default \\
\hline NR & $\mathrm{T}(5.1,18,84)$ & $\mathrm{T}(5.1,18,84)$ & $\mathrm{T}(5.1,18,84)$ & Distribution from NRC 2000 \\
\hline NR & 0.25 & 0.25 & NR & RESRAD default \\
\hline
\end{tabular}


TABLE A.1 (Cont.)

Current Use Scenarios

Future Use Scenarios

Input Parameters

Industrial Worker

Offsite Resident ${ }^{b}$

Resident Farmer $^{\mathrm{b}}$

Recreationist $^{\mathrm{c}}$

Comments

Dry foliar interception fraction or non-leafy vegetables

Wet weight crop yield for leafy vegetables $\left(\mathrm{kg} / \mathrm{m}^{2}\right)$

Length of growing season for leafy vegetables

(y)

Translocation factor for leafy vegetables

Wet foliar interception fraction for leafy

vegetables

Dry foliar interception fraction for leafy

vegetables

Wet weight crop yield for fodder $\left(\mathrm{kg} / \mathrm{m}^{2}\right)$

Length of growing season for fodder (y)

$\omega \quad$ Translocation factor for fodder

Wet foliar interception fraction for fodder

Dry foliar interception fraction for fodder

\section{Storage-Times-Before-Use Data}

Storage time for fruits, non-leafy vegetables and grain (d)

Storage time for leafy vegetables (d)

Storage time for milk (d)

Storage time for meat (d)

Storage time for fish (d)

Storage time for crustacea and mollusks (d)

Storage time for well water (d)

Storage time for surface water (d)

\section{NR}

NR

NR

NR

NR

NR

NR

NR

NR
NR

NR

NR

NR
NR

0.25
1.5

$$
0.25
$$

1
$\mathrm{~T}(0.06,0.67,0.95)$

$$
\begin{aligned}
& 0.25 \\
& \text { NR } \\
& \text { NR } \\
& \text { NR } \\
& \text { NR } \\
& \text { NR }
\end{aligned}
$$

NR

NR

\section{NR}

NR

NR

NR

NR

NR

NR

NR

7

7

1

1

14

0.25

1.5

0.25

$\mathrm{T}(0.06,0.67,0.95)$

0.25

1.1

0.08

1

0.25

0.25

14

1

20

1

1
NR

NR RESRAD default

NR RESRAD default

NR RESRAD default

NR Distribution from NRC 2000

NR RESRAD default

1.1 RESRAD default

0.08 RESRAD default

1 RESRAD default

$\begin{array}{ll}0.25 & \text { RESRAD default } \\ 0.25 & \text { RESRAD default }\end{array}$

NR

NR

NR

182.5

0

NR

$1 \quad$ NR

0

RESRAD default

RESRAD default

RESRAD default

RESRAD default for resident farmer and scenario-specific for

recreational use

RESRAD default and scenariospecific for recreational use

RESRAD default and scenariospecific for recreational use

RESRAD default for resident farmer and not required for recreational use

0 RESRAD default for resident farmer and 0 for recreational use 
TABLE A.1 (Cont.)

Current Use Scenarios

Future Use Scenarios

Input Parameters

Industrial Worker

Offsite Resident ${ }^{\mathrm{b}}$

Resident Farmer $^{\mathrm{b}}$

Recreationist $^{c}$

Comments

Storage time for livestock fodder (d)

NR

NR

\section{Radon Data}

Cover total porosity

Cover volumetric water content

Cover radon diffusion coefficient

Total porosity of the house or building

foundation

Volumetric water content of the foundation

Diffusion coefficient for radon gas in

foundation material $\left(\mathrm{m}^{2} / \mathrm{s}\right)$

$\omega$ Diffusion coefficient for radon gas in

contaminated zone soil $\left(\mathrm{m}^{2} / \mathrm{s}\right)$

Emanating power of radon-222

Radon vertical dimension of mixing (m)

Average building air exchange rate $\left(\mathrm{h}^{-1}\right)$

Height of building (room) (m)

Building indoor area factor

Bulk density of house or building foundation $\left(\mathrm{g} / \mathrm{cm}^{3}\right)$

Thickness of house or building foundation (m)

Building depth below ground surface (m)

\section{Carbon-14 Data}

$\mathrm{C}-12$ concentration in local water $\left(\mathrm{g} / \mathrm{cm}^{3}\right)$

C-12 concentration in contaminated soil $(\mathrm{g} / \mathrm{g})$

Fraction of vegetation carbon absorbed from

soil

Fraction of vegetation carbon absorbed from air

Thickness of evasion layer of C-14 in soil (m)

C-14 evasion flux rate from soil $\left(\mathrm{sec}^{-1}\right)$

\begin{tabular}{|c|c|c|c|c|}
\hline NR & NR & NR & NR & No cover \\
\hline NR & NR & NR & NR & No cover \\
\hline NR & NR & NR & NR & No cover \\
\hline 0.1 & 0.1 & 0.1 & 0.1 & RESRAD default \\
\hline 0.03 & 0.03 & 0.03 & 0.03 & RESRAD default \\
\hline $3 \mathrm{E}-07$ & $3 \mathrm{E}-07$ & $3 \mathrm{E}-07$ & $3 \mathrm{E}-07$ & RESRAD default \\
\hline $2 \mathrm{E}-06$ & 2E-06 & $2 \mathrm{E}-06$ & $2 \mathrm{E}-06$ & For site-specific soil type \\
\hline 0.28 & 0.28 & 0.28 & 0.28 & $\begin{array}{l}\text { For site-specific soil type } \\
\text { (Yu et al. 1993) }\end{array}$ \\
\hline 2.0 & 2.0 & 2.0 & 2.0 & RESRAD default \\
\hline 0.5 & 0.5 & 0.5 & 0.5 & RESRAD default \\
\hline 2.5 & 2.5 & 2.5 & 2.5 & RESRAD default \\
\hline 0 & 0 & 0 & 0 & RESRAD default \\
\hline 2.4 & 2.4 & 2.4 & 2.4 & RESRAD default \\
\hline 0.15 & 0.15 & 0.15 & 0.15 & RESRAD default \\
\hline-1 & -1 & -1 & -1 & RESRAD default \\
\hline $2 \mathrm{E}-5$ & $2 \mathrm{E}-5$ & $2 \mathrm{E}-5$ & $2 \mathrm{E}-5$ & RESRAD default \\
\hline 0.03 & 0.03 & 0.03 & 0.03 & RESRAD default \\
\hline 0.02 & 0.02 & 0.02 & 0.02 & RESRAD default \\
\hline 0.98 & 0.98 & 0.98 & 0.98 & RESRAD default \\
\hline 0.3 & 0.3 & 0.3 & 0.3 & RESRAD default \\
\hline
\end{tabular}

RESRAD default for resident farmer and scenario-specific for

recreational use

RESRAD default

or site-specific soil type

RESRAD default

ESRAD default

RESRAD default

RESRAD default 
TABLE A.1 (Cont.)

\begin{tabular}{|c|c|c|c|c|c|}
\hline \multirow[b]{2}{*}{ Input Parameters } & \multicolumn{2}{|c|}{ Current Use Scenarios } & \multicolumn{2}{|c|}{ Future Use Scenarios } & \multirow[b]{2}{*}{ Comments } \\
\hline & Industrial Worker & Offsite Resident ${ }^{\mathrm{b}}$ & Resident Farmer ${ }^{\mathrm{b}}$ & Recreationist $^{\mathrm{c}}$ & \\
\hline $\mathrm{C}-12$ evasion flux rate from soil $\left(\mathrm{sec}^{-1}\right)$ & $1 \mathrm{E}-10$ & $1 \mathrm{E}-10$ & $1 \mathrm{E}-10$ & $1 \mathrm{E}-10$ & RESRAD default \\
\hline Grain fraction in beef cattle feed & 0.8 & 0.8 & 0.8 & 0.8 & RESRAD default \\
\hline Grain fraction in milk cow feed & 0.2 & 0.2 & 0.2 & 0.2 & RESRAD default \\
\hline
\end{tabular}

a RESRAD-OFFSITE code is used for analyzing offsite resident scenario, and RESRAD code is used for other scenarios. Extra parameters required for RESRAD-OFFSITE code are listed in Table A.10.

b For offsite resident and residential farmer scenarios, the parameter values for leafy vegetable consumption, meat and poultry consumption, and fish consumption are RESRAD defaults. Parameter values for inhalation rate, indoor and outdoor time fractions, livestock fodder intake for meat, livestock water intake for meat, livestock intake of soil, plant food contaminated fraction, and meat contaminated fractions are RESRAD defaults and are calculated based on the contaminated area; storage time of meat, fish, surface water, and livestock fodder are RESRAD defaults.

c For the recreational use scenario, it is assumed that the individual would be involved in moderate activity onsite; therefore, an inhalation rate of moderate activity $\left(1.6 \mathrm{~m}^{3} / \mathrm{h}\right)$ is assumed (EPA 1997). The parameter value for meat and poultry consumption is based on the assumption that a recreationist kills a deer and brings the catch home and uses its meat over a year; the distribution is based on the game consumption statistics among households that hunt. For fish consumption, it is assumed that a recreationist catches a fish and consumes it the same day; distribution is based on the home-caught fish consumption statistics among households that fish. The parameter values for indoor and outdoor time fractions are from scenario description. All meat consumed is assumed to be contaminated. For the storage time of meat, since the meat is consumed over a year, an average storage time of 182.5 days is assumed. For the storage time of fish, since the fish is consumed on the same day, a storage time of zero days is assumed. Since the game animal uses fresh forage and surface water, a storage time of zero days is assumed for both parameters.

d NR - value is not required for this scenario.

e See Table A.8 for the notation used for the type of distribution.

f It is assumed that the soil type is silty clay at the Building 330 area (Golchert et al. 2010) and distributions representative of silty clay soil are used.

$\mathrm{g}$ Cover and management are changed to 0.0506 to get this erosion rate in RESRAD-OFFSITE code. 
TABLE A.2 Parameter Distributions Used in the Analysis - Contaminated Zone and Unsaturated Zone $\mathbf{K}_{\mathbf{d}}$ Values

\begin{tabular}{|c|c|c|c|}
\hline \multirow[b]{3}{*}{ Element } & \multicolumn{3}{|c|}{$\mathrm{K}_{\mathrm{d}}\left(\mathrm{cm}^{3} / \mathrm{g}\right)$ Distribution ${ }^{\mathrm{a}}$} \\
\hline & \multicolumn{3}{|c|}{$\begin{array}{c}\text { Lognormal Distribution } \\
\text { Parameters }\end{array}$} \\
\hline & $\mu$ & $\sigma$ & $\exp (\mu)$ \\
\hline Ac & 7.8 & $3.22^{\mathrm{b}}$ & 2,400 \\
\hline $\mathrm{Am}$ & 9.0 & 2.6 & 8,400 \\
\hline $\mathrm{Ba}^{\mathrm{c}}$ & 6.33 & 3.22 & 561 \\
\hline $\mathrm{C}$ & 0.8 & $0.8^{\mathrm{d}}$ & 1 \\
\hline Cs & 7.5 & 1.6 & 1,900 \\
\hline $\mathrm{Np}$ & 4.0 & 3.8 & 55 \\
\hline $\mathrm{Pa}$ & 7.9 & $3.22^{\mathrm{b}}$ & 2,700 \\
\hline $\mathrm{Pb}$ & 6.3 & $2.76^{\mathrm{b}}$ & 550 \\
\hline Po & 8.0 & $1.68^{\mathrm{b}}$ & 3,000 \\
\hline $\mathrm{Pu}$ & 8.5 & 2.1 & 5,100 \\
\hline $\mathrm{Ra}$ & 9.1 & 1.3 & 9,100 \\
\hline $\mathrm{Sr}$ & 4.7 & 2.0 & 110 \\
\hline Tc & 0.2 & 0.06 & 1 \\
\hline Th & 8.6 & 2.6 & 5,800 \\
\hline U & 7.3 & 2.9 & 1,600 \\
\hline
\end{tabular}

Source: Sheppard and Thibault (1990) unless noted otherwise.

a Except for Ba, the distributions are for clayey soil, which is the site-specific soil type for the contaminated zone and unsaturated zone.

b Value not available from Sheppard and Thibault (1990). The value used is default for generic soil type.

c Distribution for clayey soil is not available for $\mathrm{Ba}$; therefore, the distribution for generic soil is used.

d Value not available from Sheppard and Thibault (1990). The value used is default for sandy soil type. 
TABLE A.3 Parameter Distributions Used in the Analysis - Saturated Zone $K_{d}$ Values

\begin{tabular}{cccc}
\hline & \multicolumn{3}{c}{$\mathrm{K}_{\mathrm{d}}\left(\mathrm{cm}^{3} / \mathrm{g}\right)$ Distribution ${ }^{\mathrm{a}}$} \\
\cline { 2 - 4 } & \multicolumn{3}{c}{ Lognormal Distribution } \\
& \multicolumn{3}{c}{ Parameters } \\
\cline { 2 - 4 } Element & $\mu$ & $\sigma$ & $\exp (\mu)$ \\
\hline & & & \\
$\mathrm{Ac}$ & 6.1 & $3.22^{\mathrm{b}}$ & 450 \\
$\mathrm{Am}$ & 7.6 & 2.6 & 1,900 \\
$\mathrm{Ba}$ & 6.33 & 3.22 & 561 \\
$\mathrm{C}$ & 1.1 & 0.8 & 5 \\
$\mathrm{Cs}$ & 5.6 & 2.5 & 280 \\
$\mathrm{~Np}$ & 1.4 & 1.7 & 5 \\
$\mathrm{~Pa}$ & 6.3 & $3.22^{\mathrm{b}}$ & 550 \\
$\mathrm{~Pb}$ & 5.6 & $2.76^{\mathrm{b}}$ & 270 \\
$\mathrm{Po}$ & 5.0 & 1.6 & 150 \\
$\mathrm{Pu}$ & 6.3 & 1.7 & 550 \\
$\mathrm{Ra}$ & 6.2 & 3.2 & 500 \\
$\mathrm{Sr}$ & 2.6 & 1.6 & 15 \\
$\mathrm{Tc}$ & -2.0 & 1.8 & 0.1 \\
$\mathrm{Th}$ & 8.0 & 2.1 & 3,200 \\
$\mathrm{U}$ & 3.5 & 3.2 & 35 \\
\hline
\end{tabular}

Source: Sheppard and Thibault (1990) unless noted otherwise.

a Except for $\mathrm{Ba}$, the distributions are for sandy soil, which is assumed to be the soil type for the saturated zone.

b Value not available from Sheppard and Thibault (1990). The value used is default for generic soil type.

c Distribution for sandy soil is not available for $\mathrm{Ba}$; therefore, the distribution for generic soil is used. 
TABLE A.4 Parameter Distributions Used in the Analysis for the Plant Transfer Factor

\begin{tabular}{cccc}
\hline & \multicolumn{3}{c}{$\begin{array}{c}\text { Plant Transfer Factor (wet plant } \\
\text { concentration, pCi/g/dry soil } \\
\text { concentration, pCi/g) Distribution }\end{array}$} \\
\cline { 2 - 4 } & \multicolumn{3}{c}{ Lognormal Distribution } \\
& \multicolumn{3}{c}{ Parameters } \\
\cline { 2 - 4 } Element & $\mu$ & $\sigma$ & exp $(\mu)$ \\
\hline & \multicolumn{3}{c}{} \\
$\mathrm{Ac}$ & -6.91 & 1.1 & $1.0 \times 10^{-3}$ \\
$\mathrm{Am}$ & -6.91 & 0.9 & $1.0 \times 10^{-3}$ \\
$\mathrm{Ba}$ & -4.61 & 1.1 & $1.0 \times 10^{-2}$ \\
$\mathrm{C}$ & -0.36 & 0.9 & $7.0 \times 10^{-1}$ \\
$\mathrm{Cs}$ & -3.22 & 1.0 & $4.0 \times 10^{-2}$ \\
$\mathrm{~Np}$ & -3.91 & 0.9 & $2.0 \times 10^{-2}$ \\
$\mathrm{~Pa}$ & -4.61 & 1.1 & $1.0 \times 10^{-2}$ \\
$\mathrm{~Pb}$ & -5.52 & 0.9 & $4.0 \times 10^{-3}$ \\
$\mathrm{Po}$ & -6.9 & 0.9 & $1.0 \times 10^{-3}$ \\
$\mathrm{Pu}$ & -6.91 & 0.9 & $1.0 \times 10^{-3}$ \\
$\mathrm{Ra}$ & -3.22 & 0.9 & $4.0 \times 10^{-2}$ \\
$\mathrm{Sr}$ & -1.20 & 1.0 & $3.0 \times 10^{-1}$ \\
$\mathrm{Tc}$ & 1.61 & 0.9 & $5.0 \times 10^{0}$ \\
$\mathrm{Th}$ & -6.91 & 0.9 & $1.0 \times 10^{-3}$ \\
$\mathrm{U}$ & -6.21 & 0.9 & $2.0 \times 10^{-3}$ \\
\hline
\end{tabular}

Source: NRC 2000. 
TABLE A.5 Parameter Distribution

Used in the Analysis for the Meat

Transfer Factor

\begin{tabular}{|c|c|c|c|}
\hline \multirow[b]{3}{*}{ Element } & \multicolumn{3}{|c|}{$\begin{array}{c}\text { Meat Transfer Factor } \\
\text { (concentration in meat, } \mathrm{pCi} / \mathrm{g} / \mathrm{rate} \\
\text { of intake, } \mathrm{pCi} / \mathrm{d} \text { ) Distribution }\end{array}$} \\
\hline & \multicolumn{3}{|c|}{$\begin{array}{c}\text { Lognormal Distribution } \\
\text { Parameters }\end{array}$} \\
\hline & $\mu$ & $\sigma$ & $\exp (\mu)$ \\
\hline $\mathrm{Ac}$ & -6.91 & 1.1 & $1.0 \times 10^{-3}$ \\
\hline $\mathrm{Am}$ & -9.90 & 0.2 & $5.0 \times 10^{-5}$ \\
\hline $\mathrm{Ba}$ & -8.52 & 0.9 & $2.0 \times 10^{-4}$ \\
\hline $\mathrm{C}$ & -3.47 & 1 & $3.1 \times 10^{-2}$ \\
\hline Cs & -3.00 & 0.4 & $5.0 \times 10^{-2}$ \\
\hline $\mathrm{Np}$ & -6.91 & 0.7 & $1.0 \times 10^{-3}$ \\
\hline $\mathrm{Pa}$ & -12.21 & 1.0 & $5.0 \times 10^{-6}$ \\
\hline $\mathrm{Pb}$ & -7.13 & 0.7 & $8.0 \times 10^{-4}$ \\
\hline Po & -5.30 & 0.7 & $5.0 \times 10^{-3}$ \\
\hline $\mathrm{Pu}$ & -9.21 & 0.2 & $1.0 \times 10^{-4}$ \\
\hline $\mathrm{Ra}$ & -6.91 & 0.7 & $1.0 \times 10^{-3}$ \\
\hline $\mathrm{Sr}$ & -4.61 & 0.4 & $1.0 \times 10^{-2}$ \\
\hline $\mathrm{Tc}$ & -9.21 & 0.7 & $1.0 \times 10^{-4}$ \\
\hline Th & -9.21 & 1.0 & $1.0 \times 10^{-4}$ \\
\hline $\mathrm{U}$ & -7.13 & 0.7 & $8.0 \times 10^{-4}$ \\
\hline
\end{tabular}

Source: NRC 2000. 
TABLE A.6 Parameter Distribution Used in the Analysis for the Milk

\section{Transfer Factor}

\begin{tabular}{|c|c|c|c|}
\hline \multirow[b]{3}{*}{ Element } & \multicolumn{3}{|c|}{$\begin{array}{c}\text { Milk Transfer Factor } \\
\text { (concentration in milk, pCi/L/rate } \\
\text { of intake, } \mathrm{pCi} / \mathrm{d} \text { ) Distribution }\end{array}$} \\
\hline & \multicolumn{3}{|c|}{$\begin{array}{c}\text { Lognormal Distribution } \\
\text { Parameters } \\
\end{array}$} \\
\hline & $\mu$ & $\sigma$ & $\exp (\mu)$ \\
\hline Ac & -13.12 & 0.9 & $2.0 \times 10^{-6}$ \\
\hline $\mathrm{Am}$ & -13.12 & 0.7 & $2.0 \times 10^{-6}$ \\
\hline $\mathrm{Ba}$ & -7.6 & 0.7 & $5.0 \times 10^{-4}$ \\
\hline $\mathrm{C}$ & -4.4 & 0.9 & $1.2 \times 10^{-2}$ \\
\hline Cs & -4.61 & 0.5 & $1.0 \times 10^{-2}$ \\
\hline $\mathrm{Np}$ & -11.51 & 0.7 & $1.0 \times 10^{-5}$ \\
\hline $\mathrm{Pa}$ & -12.21 & 0.9 & $5.0 \times 10^{-6}$ \\
\hline $\mathrm{Pb}$ & -8.11 & 0.9 & $3.0 \times 10^{-4}$ \\
\hline Po & -7.82 & 0.7 & $4.0 \times 10^{-4}$ \\
\hline $\mathrm{Pu}$ & -13.82 & 0.5 & $1.0 \times 10^{-6}$ \\
\hline $\mathrm{Ra}$ & -6.91 & 0.5 & $1.0 \times 10^{-3}$ \\
\hline $\mathrm{Sr}$ & -6.21 & 0.5 & $2.0 \times 10^{-3}$ \\
\hline Tc & -6.91 & 0.7 & $1.0 \times 10^{-3}$ \\
\hline Th & -12.21 & 0.9 & $5.0 \times 10^{-6}$ \\
\hline $\mathrm{U}$ & -7.82 & 0.6 & $4.0 \times 10^{-4}$ \\
\hline
\end{tabular}

Source: NRC 2000. 
TABLE A.7 Parameter Value/Distribution Used in the Analysis for the Fish and Crustacea Transfer Factor

\begin{tabular}{|c|c|c|c|c|}
\hline \multirow[b]{3}{*}{ Element } & \multirow{3}{*}{$\begin{array}{c}\text { Crustacea Transfer } \\
\text { Factor (concentration } \\
\text { in crustacea, } \mathrm{pCi} / \mathrm{Kg} / \\
\text { concentration in water, } \\
\text { pCi/L) }\end{array}$} & \multirow{2}{*}{\multicolumn{3}{|c|}{$\begin{array}{c}\text { Fish Transfer Factor } \\
\text { (concentration in fish, } \\
\text { pCi/Kg/concentration in water, } \\
\text { pCi/L) Distributions } \\
\begin{array}{c}\text { Lognormal Distribution } \\
\text { Parameters }\end{array} \\
\end{array}$}} \\
\hline & & & & \\
\hline & & $\mu$ & $\sigma$ & $\exp (\mu)$ \\
\hline Ac & 1,000 & 2.7 & 1.1 & 15 \\
\hline Am & 1,000 & 3.4 & 1.1 & 30 \\
\hline $\mathrm{Ba}$ & 200 & 1.4 & 1.1 & 4 \\
\hline C & 9,100 & 10.8 & 1.1 & 50,000 \\
\hline Cs & 100 & 7.6 & 0.7 & 2,000 \\
\hline $\mathrm{Np}$ & 400 & 3.4 & 1.1 & 30 \\
\hline $\mathrm{Pa}$ & 110 & 2.3 & 1.1 & 10 \\
\hline $\mathrm{Pb}$ & 100 & 5.7 & 1.1 & 300 \\
\hline Po & 20,000 & 4.6 & 1.1 & 100 \\
\hline $\mathrm{Pu}$ & 100 & 3.4 & 1.1 & 30 \\
\hline $\mathrm{Ra}$ & 250 & 3.9 & 1.1 & 50 \\
\hline $\mathrm{Sr}$ & 100 & 4.1 & 1.1 & 60 \\
\hline $\mathrm{Tc}$ & 5 & 3.0 & 1.1 & 20 \\
\hline Th & 500 & 4.6 & 1.1 & 100 \\
\hline $\mathrm{U}$ & 60 & 2.3 & 1.1 & 10 \\
\hline
\end{tabular}

Source: NRC 2000. 
TABLE A.8 Notations Used in Input Parameter Table 1

\begin{tabular}{ll}
\hline \multicolumn{1}{c}{ Notation } & \multicolumn{1}{c}{ Type of Distribution } \\
\hline $\mathrm{TN}(\mu, \sigma, \mathrm{a}, \mathrm{b})^{\mathrm{a}}$ & Truncated Normal \\
$\mathrm{TLN}(\mu, \sigma, \mathrm{a}, \mathrm{b})^{\mathrm{b}}$ & Truncated Lognormal-N \\
$\mathrm{BLN}(\mu, \sigma, \mathrm{a}, \mathrm{b})^{\mathrm{c}}$ & Bounded Lognormal-N \\
$\mathrm{U}(\mathrm{a}, \mathrm{b})^{\mathrm{d}}$ & Uniform \\
$\mathrm{LU}(\mathrm{a}, \mathrm{b})^{\mathrm{e}}$ & Log Uniform \\
$\mathrm{T}(\mathrm{a}, \mathrm{c}, \mathrm{b})^{\mathrm{f}}$ & Triangular \\
Continuous linear $^{\mathrm{g}}$ & Empirical \\
Continuous log $^{\mathrm{h}}$ & Empirical \\
\hline
\end{tabular}

a For truncated normal distribution, $\mu$ is mean, $\sigma$ is standard deviation, a is lower quantile, and $b$ is upper quantile of the distribution.

b For truncated lognormal-N distribution, $\mu$ is mean and $\sigma$ is standard deviation of underlying normal distribution; and a is lower quantile, and $b$ is upper quantile of the distribution.

c For bounded lognormal-N distribution, $\mu$ is mean and $\sigma$ is standard deviation of underlying normal distribution; and a is minimum, and $b$ is maximum of the distribution.

d For uniform distribution, $a$ and $b$ parameters represent the minimum and maximum values, respectively.

e For log uniform distribution, $a$ and $b$ parameters represent the minimum and maximum values, respectively.

f For triangular distribution, a is minimum, $\mathrm{c}$ is mode, and $\mathrm{b}$ is maximum of the distribution.

g The continuous linear distribution is defined by the user for the $\mathrm{N}$ points, and the distribution is assumed to vary linearly between the cumulative distribution functions specified by the user for the $\mathrm{N}$ points.

$\mathrm{h}$ The continuous log distribution is defined by the user for the $\mathrm{N}$ points, and the distribution is assumed to vary logarithmically between the cumulative distribution functions specified by the user for the $\mathrm{N}$ points. 


\section{TABLE A.9 Parameter Correlations for Probabilistic Analyses in Different Scenarios}

\begin{tabular}{|c|c|c|c|}
\hline Parameter 1 & Parameter 2 & $\begin{array}{l}\text { Correlation } \\
\text { Coefficient }\end{array}$ & Comments \\
\hline Contaminated zone soil density & $\begin{array}{l}\text { Contaminated zone } \\
\text { total porosity }\end{array}$ & -0.99 & $\begin{array}{l}\text { The two parameters are strongly } \\
\text { negatively correlated }\end{array}$ \\
\hline Unsaturated zone soil density & $\begin{array}{l}\text { Unsaturated zone } \\
\text { total porosity }\end{array}$ & -0.99 & $\begin{array}{l}\text { The two parameters are strongly } \\
\text { negatively correlated }\end{array}$ \\
\hline Unsaturated zone soil density & $\begin{array}{l}\text { Unsaturated zone } \\
\text { effective porosity }\end{array}$ & -0.99 & $\begin{array}{l}\text { The two parameters are strongly } \\
\text { negatively correlated }\end{array}$ \\
\hline Saturated zone soil density & $\begin{array}{l}\text { Saturated zone total } \\
\text { porosity }\end{array}$ & -0.99 & $\begin{array}{l}\text { The two parameters are strongly } \\
\text { negatively correlated }\end{array}$ \\
\hline Saturated zone soil density & $\begin{array}{l}\text { Saturated zone } \\
\text { effective porosity }\end{array}$ & -0.99 & $\begin{array}{l}\text { The two parameters are strongly } \\
\text { negatively correlated }\end{array}$ \\
\hline Unsaturated zone total porosity & $\begin{array}{l}\text { Unsaturated zone } \\
\text { effective porosity }\end{array}$ & 0.99 & $\begin{array}{l}\text { A correlation of } 0.99 \text { provides } \\
\text { satisfactory pairing of sampling data }\end{array}$ \\
\hline Saturated zone total porosity & $\begin{array}{l}\text { Saturated zone } \\
\text { effective porosity }\end{array}$ & 0.99 & $\begin{array}{l}\text { A correlation of } 0.99 \text { provides } \\
\text { satisfactory pairing of sampling data }\end{array}$ \\
\hline
\end{tabular}




\section{TABLE A.10 Additional Input Parameters for RESRAD-OFFSITE Analysis}

Parameter

Site Layout ${ }^{\mathrm{a}}$

$\mathrm{X}$ dimension of primary contamination (m)

$\mathrm{Y}$ dimension of primary contamination $(\mathrm{m})$

Smaller X coordinate of the fruit, grain, non-leafy

vegetables plot $(\mathrm{m})$

Larger X coordinate of the fruit, grain, non-leafy vegetables plot $(\mathrm{m})$

Smaller Y coordinate of the fruit, grain, non-leafy vegetables plot $(\mathrm{m})$

Larger $Y$ coordinate of the fruit, grain, non-leafy vegetables plot $(\mathrm{m})$

Smaller X coordinate of the leafy vegetables plot (m)

Larger X coordinate of the leafy vegetables plot (m)

Smaller Y coordinate of the leafy vegetables plot (m)

Larger Y coordinate of the leafy vegetables plot (m)

Smaller X coordinate of the pasture, silage growing area $(\mathrm{m})$

Larger X coordinate of the pasture, silage growing area $(\mathrm{m})$

Smaller Y coordinate of the pasture, silage growing area $(\mathrm{m})$

Larger $\mathrm{Y}$ coordinate of the pasture, silage growing area $(\mathrm{m})$

Smaller X coordinate of the grain fields $(\mathrm{m})$

Larger X coordinate of the grain fields (m)

Smaller Y coordinate of the grain fields $(\mathrm{m})$

Larger $\mathrm{Y}$ coordinate of the grain fields (m)

Smaller X coordinate of the dwelling site (m)

Larger X coordinate of the dwelling site (m)

Smaller Y coordinate of the dwelling site $(\mathrm{m})$

Larger $Y$ coordinate of the dwelling site $(\mathrm{m})$

Smaller X coordinate of the surface water body

Larger X coordinate of the surface water body

Value

Comment

100

100

434.4

465.6

434

466

434.4

465.6

468

500

450

550

500

600

500

600

600

700

434.4

465.6

400

432

526.5

826.5
Total contaminated area $=10,000 \mathrm{~m}^{2}$

Closest distance to the site boundary

Assume the vegetable plot is $31.6 \mathrm{~m} \times$ $31.6 \mathrm{~m}$, with an area of $1,000 \mathrm{~m}^{2}$

Closest distance to the site boundary

Assume the vegetable plot is $31.6 \mathrm{~m} \times$ $31.6 \mathrm{~m}$, with an area of $1,000 \mathrm{~m}^{2}$

Closest distance to the site boundary

Assume the vegetable plot is $31.6 \mathrm{~m} \times$ $31.6 \mathrm{~m}$, with an area of $1,000 \mathrm{~m}^{2}$

Leafy vegetable plot is assumed to be next to the nonleafy vegetable plot

Assume the vegetable plot is $31.6 \mathrm{~m} \times$ $31.6 \mathrm{~m}$, with an area of $1,000 \mathrm{~m}^{2}$

Close to the vegetable plots

Assume the livestock feed area is $100 \mathrm{~m} \times 100 \mathrm{~m}$, with an area of $10,000 \mathrm{~m}^{2}$

Close to the vegetable plots

Assume the livestock feed area is $100 \mathrm{~m}$ $\times 100 \mathrm{~m}$, with an area of $10,000 \mathrm{~m}^{2}$

Close to the vegetable plots

Assume the livestock feed area is $100 \mathrm{~m}$ $\times 100 \mathrm{~m}$, with an area of $10,000 \mathrm{~m}^{2}$

Close to the vegetable plots

Assume the livestock feed area is $100 \mathrm{~m}$ $\times 100 \mathrm{~m}$, with an area of $10,000 \mathrm{~m}^{2}$

Closest distance to the site boundary

The dwelling site is assumed to be

$31.6 \mathrm{~m} \times 31.6 \mathrm{~m}$ and have an area of $1,000 \mathrm{~m}^{2}$

Closest distance to the site boundary

The dwelling site is assumed to be $31.6 \mathrm{~m} \times 31.6 \mathrm{~m}$ and have an area of $1,000 \mathrm{~m}^{2}$

The surface water body is assumed to be close to the dwelling site and the vegetable and livestock feed areas

The surface water body is assumed to be close to the dwelling site and the vegetable and livestock feed areas 
TABLE A.10 (Cont.)

\begin{tabular}{|c|c|c|}
\hline Parameter & Value & Comment \\
\hline Smaller Y coordinate of the surface water body & 356.8 & $\begin{array}{l}\text { The surface water body is assumed to } \\
\text { be close to the dwelling site and the } \\
\text { vegetable and livestock feed areas }\end{array}$ \\
\hline Larger Y coordinate of the surface water body & 656.8 & $\begin{array}{l}\text { The surface water body is assumed to } \\
\text { be close to the dwelling site and the } \\
\text { vegetable and livestock feed areas }\end{array}$ \\
\hline \multicolumn{3}{|l|}{ Source Release and Deposition Velocity } \\
\hline Deposition velocity $(\mathrm{m} / \mathrm{s})$ & 0.001 & $\begin{array}{l}\text { For all radionuclides except I-129 } \\
\quad(0.01)\end{array}$ \\
\hline \multicolumn{3}{|l|}{ Distribution Coefficients } \\
\hline Sediment in surface water body $\left(\mathrm{cm}^{3} / \mathrm{g}\right)$ & Table A.2 & \\
\hline Fruit, grain, non-leafy vegetable fields $\left(\mathrm{cm}^{3} / \mathrm{g}\right)$ & Table A.2 & \\
\hline Leafy vegetable fields $\left(\mathrm{cm}^{3} / \mathrm{g}\right)$ & Table A.2 & \\
\hline Pasture, silage growing areas $\left(\mathrm{cm}^{3} / \mathrm{g}\right)$ & Table A. 2 & \\
\hline Livestock feed grain fields $\left(\mathrm{cm}^{3} / \mathrm{g}\right)$ & Table A.2 & \\
\hline Offsite dwelling site $\left(\mathrm{cm}^{3} / \mathrm{g}\right)$ & Table A.2 & \\
\hline \multicolumn{3}{|l|}{ Transfer Factors } \\
\hline Fruit, grain, nonleafy vegetable transfer factor & Table A.3 & \\
\hline Leafy vegetable transfer factor & Table A.3 & \\
\hline Pasture and silage transfer factor & Table A.3 & \\
\hline Livestock feed grain transfer factor & Table A.3 & \\
\hline \multicolumn{3}{|l|}{ Storage Time } \\
\hline Storage time for pasture and silage (d) & 1 & Default \\
\hline Storage time for livestock feed grain (d) & 45 & Default \\
\hline \multicolumn{3}{|l|}{ Primary Contamination } \\
\hline Deposition velocity of dust $(\mathrm{m} / \mathrm{s})$ & 0.001 & Default \\
\hline Rainfall and runoff factor & 160 & Default \\
\hline Slope-length-steepness factor & 0.4 & Default \\
\hline Cover and management factor & 0.0506 & $\begin{array}{l}\text { Adjusted to match site-specific erosion } \\
\text { rate }\end{array}$ \\
\hline Support practice factor & 1 & Default \\
\hline \multicolumn{3}{|l|}{ Contaminated Zone } \\
\hline Soil erodibility factor of contaminated zone & 0.4 & Default \\
\hline \multicolumn{3}{|l|}{ Clean Cover } \\
\hline Soil erodibility factor of clean cover & NR & No cover \\
\hline Volumetric water content of clean cover & NR & No cover \\
\hline \multicolumn{3}{|l|}{$\begin{array}{l}\text { Agriculture/Livestock Feed Growing/Offsite } \\
\text { Dwelling Area Parameters }\end{array}$} \\
\hline $\begin{array}{l}\text { Fraction of area directly over primary contamination } \\
\text { for all fields }\end{array}$ & 0 & Receptor offsite \\
\hline
\end{tabular}




\begin{tabular}{|c|c|c|}
\hline Parameter & Value & Comment \\
\hline Irrigation applied per year for all fields (m/y) & 0.1 & Site specific \\
\hline Evapotranspiration coefficient for all fields & $\mathrm{U}(0.5,0.75)$ & Distribution \\
\hline Runoff coefficient for all fields & $\mathrm{U}(0.1,0.8)$ & Distribution \\
\hline $\begin{array}{l}\text { Depth of soil mixing layer or plow layer for all fields } \\
\text { (m) }\end{array}$ & See Table A.1 & \\
\hline Volumetric water content for all fields & 0.3 & Default \\
\hline Dry bulk density of soil for all fields $\left(\mathrm{g} / \mathrm{cm}^{3}\right)$ & $\begin{array}{c}\mathrm{TN}^{\mathrm{d}}(1.696, \\
0.1855,0.001 \\
0.999)\end{array}$ & $\begin{array}{l}\text { Site-specific soil type (silty clay - } \\
\text { Golchert et al. 2010) distribution from } \\
\text { NRC } 2000\end{array}$ \\
\hline Soil erodibility factor for all fields & 0.4 & Default \\
\hline Slope-length-steepness factor for all fields & 0.4 & Default \\
\hline Cover and management factor for all fields & 0.0506 & $\begin{array}{l}\text { Adjusted to match site-specific erosion } \\
\text { rate }\end{array}$ \\
\hline Support practice factor for all fields & 1 & Default \\
\hline \multicolumn{3}{|l|}{ Atmospheric Transport } \\
\hline Release height (m) & 1 & Default \\
\hline Release heat flux (cal/s) & 0 & Default \\
\hline Anemometer height (m) & 10 & Default \\
\hline Ambient temperature $(\mathrm{K})$ & 285 & Default \\
\hline AM atmospheric mixing height $(\mathrm{m})$ & 400 & Default \\
\hline PM atmospheric mixing height (m) & 1,600 & Default \\
\hline Dispersion model coefficients & $\begin{array}{l}\text { Pasquill- } \\
\text { Gifford }\end{array}$ & Default \\
\hline Wind speed terrain & Rural & Default \\
\hline $\begin{array}{l}\text { Elevation of off-site location, relative to ground level } \\
\text { at primary contamination, for all fields (m) }\end{array}$ & 0 & Default \\
\hline Grid spacing for areal integration $(\mathrm{m})$ & 10 & Default \\
\hline $\begin{array}{l}\text { Joint frequency of wind speed and stability class for } \\
\text { a 16-sector wind rose }\end{array}$ & $\begin{array}{l}\text { Actual values } \\
\text { from Chicago } \\
\text { Midway }\end{array}$ & Site-specific \\
\hline \multicolumn{3}{|l|}{ Unsaturated Zone Parameters } \\
\hline Unsaturated zone longitudinal dispersivity (m) & 0.1 & Default \\
\hline \multicolumn{3}{|l|}{ Saturated Zone Hydrological Data } \\
\hline Thickness of saturated zone (m) & 18.3 & Default \\
\hline $\begin{array}{l}\text { Saturated zone longitudinal dispersivity to surface } \\
\text { water body }(\mathrm{m})\end{array}$ & 10 & Default \\
\hline $\begin{array}{l}\text { Saturated zone horizontal lateral dispersivity to } \\
\text { surface water body }(\mathrm{m})\end{array}$ & 1 & Default \\
\hline Disperse vertically & Check box & Yes \\
\hline $\begin{array}{l}\text { Saturated zone vertical lateral dispersivity to surface } \\
\text { water body }(\mathrm{m})\end{array}$ & .06 & Default \\
\hline $\begin{array}{l}\text { Depth of aquifer contributing to surface water body } \\
\text { (m) }\end{array}$ & 10 & Default \\
\hline
\end{tabular}




\section{TABLE A.10 (Cont.)}

Parameter

Surface Water Body

Sediment deliver ratio

Volume of surface water body $\left(\mathrm{m}^{3}\right)$

Mean residence time of water in surface water body (y)

\section{Groundwater Transport Parameters}

Distance from downgradient edge of contamination to well in the direction parallel to aquifer flow (m)

Distance from downgradient edge of contamination to surface water body in the direction parallel to aquifer flow (m)

Distance from downgradient edge of contamination to well in the direction perpendicular to aquifer flow (m)

Distance from downgradient edge of contamination to the right edge of surface water body in the direction perpendicular to aquifer flow (m)

Distance from downgradient edge of contamination to the left edge of the surface water body in the direction perpendicular to aquifer flow (m)

Main subzones in saturated zone

Main subzones in each partially saturated zone

Nuclide-specific retardation in all subzones, longitudinal dispersion in all but the subzone of transformation?

\section{Water Use}

Quantity of water consumed by an individual (L/yr)

Number of household individuals consuming and using water

Quantity of water for use indoors of dwelling per individual $(\mathrm{L} / \mathrm{d})$

Quantity of water for beef cattle (L/d)

Number of beef cattle

Quantity of water for dairy cows (L/d)

Number of dairy cows

Well pumping rate $\left(\mathrm{m}^{3} / \mathrm{yr}\right)$

\section{Ingestion Rates}

Drinking water intake from affected area

Fish intake from affected area

Crustacea and mollusks intake from affected area

Fruit, grain, non-leafy vegetables fraction from affected area

Leafy vegetables fraction from affected area

Meat fraction from affected area
Value

Comment

$\begin{array}{cc}1 & \text { Default } \\ 150,000 & \text { Default } \\ 1 & \text { Default }\end{array}$

$-55 \quad$ Site-specific

$256.8 \quad$ Site-specific

$-424 \quad$ Site-specific

$-150 \quad$ Default

$150 \quad$ Default

$\begin{array}{cc}1 & \text { Default } \\ 1 & \text { Default } \\ \text { Yes } & \text { Default }\end{array}$

See Table A.1 Household water not contaminated $4 \quad$ Default

225 Household water not contaminated

50 Default

2 Default

160 Default

2 Default

250 RESRAD default

$\begin{array}{cl}0 & \text { Drinking water not contaminated } \\ 0.5 & \text { Default } \\ 0.5 & \text { Default } \\ 0.5 & \text { Default } \\ & \\ 0.5 & \text { Default } \\ 0.5 & 50 \% \text { of the meat consumed is from }\end{array}$
contaminated area 


\section{TABLE A.10 (Cont.)}

\begin{tabular}{|c|c|c|}
\hline Parameter & Value & Comment \\
\hline Milk fraction from affected area & 0.5 & $\begin{array}{l}50 \% \text { of the meat consumed is from } \\
\text { contaminated area }\end{array}$ \\
\hline \multicolumn{3}{|l|}{ Livestock intake } \\
\hline Pasture and silage intake for beef cattle $(\mathrm{kg} / \mathrm{d})$ & 14 & Default \\
\hline Grain intake for beef cattle $(\mathrm{kg} / \mathrm{d})$ & 54 & Default \\
\hline $\begin{array}{l}\text { Soil from pasture and silage intake for beef cattle } \\
(\mathrm{kg} / \mathrm{d})\end{array}$ & 0.1 & Default \\
\hline Soil from grain intake for beef cattle $(\mathrm{kg} / \mathrm{d})$ & 0.4 & Default \\
\hline Pasture and silage intake for dairy cows $(\mathrm{kg} / \mathrm{d})$ & 44 & Default \\
\hline Grain intake for dairy cows $(\mathrm{kg} / \mathrm{d})$ & 11 & Default \\
\hline $\begin{array}{l}\text { Soil from pasture and silage intake for dairy cows } \\
(\mathrm{kg} / \mathrm{d})\end{array}$ & 0.4 & Default \\
\hline Soil from grain intake for dairy cows $(\mathrm{kg} / \mathrm{d})$ & 0.1 & Default \\
\hline \multicolumn{3}{|l|}{$\begin{array}{l}\text { Livestock Feed Factors (for Pasture and } \\
\text { Silage, Grain) }\end{array}$} \\
\hline Wet weight crop yield $\left(\mathrm{kg} / \mathrm{m}^{2}\right)$ & $1.1,0.7$ & Default \\
\hline Duration of growing season $(\mathrm{yr})$ & $0.08,0.17$ & Default \\
\hline Foliage to food transfer coefficient & $1,0.1$ & Default \\
\hline Weathering removal constant & 20,20 & Default \\
\hline Foliar interception factor for irrigation & $0.25,0.25$ & Default \\
\hline Foliar interception factor for dust deposition & $0.25,0.25$ & Default \\
\hline Root depth (m) & See Table A.1 & $\begin{array}{l}\text { Distribution from NUREG/CR-6697 } \\
\text { (NRC 2000) }\end{array}$ \\
\hline \multicolumn{3}{|l|}{ Occupancy Factors } \\
\hline Indoor time fraction on primary contamination & 0 & \\
\hline Outdoor time fraction on primary contamination & 0 & \\
\hline Indoor time fraction on offsite dwelling site & 0.5 & \\
\hline Outdoor time fraction on offsite dwelling site & 0.1 & \\
\hline $\begin{array}{l}\text { Time fraction in fruit, grain, and non-leafy vegetable } \\
\text { fields }\end{array}$ & 0.1 & \\
\hline Time fraction in leafy vegetable fields & 0.1 & \\
\hline Time fraction in pasture and silage fields & 0.1 & \\
\hline Time fraction in livestock grain fields & 0.1 & \\
\hline
\end{tabular}

a All the areas (dwelling, agricultural areas) are assumed to be less than $350 \mathrm{~m}$ away from the contaminated area. This is a conservative assumption because the distance to the site boundary from the Building 330 area is greater than $350 \mathrm{~m}$. 


\section{REFERENCES}

ANL (Argonne National Laboratory), 1996, Interim Action Documentation Report:

Decontamination and Demolition of the 317 Area South Vaults, Argonne National Laboratory, Argonne Ill., May 31.

Eckerman, K.F., and J.C. Ryman, 1993, External Exposure to Radionuclides in Air, Water, and Soil, Exposure to Dose Coefficients for General Application, Based on the 1987 Federal Radiation Protection Guidance, EPA 402-R-93-076, Federal Guidance Report No. 12, prepared by Oak Ridge National Laboratory, Oak Ridge, Tenn., for U.S. Environmental Protection Agency, Office of Radiation and Indoor Air, Washington, D.C.

EPA (U.S. Environmental Protection Agency), 1997, Exposure Factor Handbook, EPA/600/P-95/002Fa, Office of Research and Development, National Center for Environmental Assessment, Washington, D.C.

Golchert, N.W., T.M. Davis, and L.P. Moos, 2010, Argonne Site Environmental Report for Calendar Year 2009, ANL-10/02, Argonne National Laboratory, Argonne, Ill., September.

ICRP (International Commission on Radiological Protection), 1991, 1990 Recommendations of the International Commission on Radiological Protection, ICRP Publication 60, Ann. ICRP 21(1-3), Pergamon Press, Oxford, England.

ICRP, 1996, Age-Dependent Doses to Members of the Public from Intake of Radionuclides: Part 5, Compilation of Ingestion and Inhalation Coefficients, ICRP Publication 72, Ann. ICRP 26(1), Pergamon Press, Oxford, England.

Kamboj, S., 2011, personal communication between S. Kamboj, Argonne, and R.L. Johnson, Argonne, Subject: "Bldg. 310 RESRAD Analysis Project Status," January 7.

Matton, P.B., 2011, personal communication between P.B. Matton, Argonne, and R.J. Johnson, Argonne, Subject: "Bldg. 330 D\&D Project-RESRAD, DCGLs, Clean-up Parameters," January 14.

NCDC (National Climatic Data Center), 2010, “Average Wind Speed.” Available at http://lwf.ncdc.noaa.gov/oa/climate/online/ccd/avgwind.html.

NRC (U.S. Nuclear Regulatory Commission), 2000, Development of Probabilistic RESRAD 6.0 and RESRAD-BUILD 3.0 Computer Codes, NUREG/CR-6697 and ANL/EAD/TM-98, prepared by C. Yu et al., Argonne National Laboratory, Argonne, Ill., for U.S. Nuclear Regulatory Commission, Washington, D.C., Nov.

NRCS (Natural Resources Conservation Service), 2010, "Report Cards Are Out - Illinois NRI Gets Gold Star!," news release, May 25. 
Patton, T.L., R.H. Pearl, and S.Y. Tsai, 1990, Hydrological Conditions at the 800 Area at Argonne National Laboratory, ANL/EAIS/TM-29, Argonne National Laboratory, Argonne, Ill., August.

Quinn, J., 2010, personal communication between J. Quinn, Argonne, and S. Kamboj, Argonne, November 12.

Sheppard, M.I. and D.H. Thibault, 1990, "Default Soil Solid/Liquid Partition Coefficients, Kds, for Four Major Soil Types: A Compendium,” Health Physics 59(4):471-482.

Yu, C., et al., 1993, Data Collection Handbook to Support Modeling the Impacts of Radioactive Material in Soil, ANL/EAIS-8, Argonne National Laboratory, Argonne, Ill., April.

Yu, C., et al., 2001, User's Manual for RESRAD Version 6, ANL/EAD-4, Argonne National Laboratory, Argonne, Ill. 


\section{APPENDIX B:}

\section{PARAMETERS USED FOR DETERMINISTIC ANALYSIS}

Tables B.1-B.4 list all input parameter distributions or parameter values used in the deterministic dose analysis for different user scenarios. 


\section{TABLE B.1 Parameter Values Used for Deterministic Analysis of Different Scenarios ${ }^{\text {a }}$}

\begin{tabular}{|c|c|c|c|c|c|}
\hline \multirow[b]{2}{*}{ Input Parameters } & \multicolumn{2}{|c|}{ Current Use Scenarios } & \multicolumn{2}{|c|}{ Future Use Scenarios } & \multirow[b]{2}{*}{ Comments } \\
\hline & Industrial Worker & Offsite Resident ${ }^{\mathrm{b}}$ & Resident Farmer ${ }^{\mathrm{b}}$ & Recreationist $\mathrm{t}^{\mathrm{c}}$ & \\
\hline \multicolumn{6}{|l|}{ Title } \\
\hline Title & Scenario dependent & Scenario dependent & Scenario dependent & Scenario dependent & Scenario definition \\
\hline Dose factor library & ICRP-60 & ICRP-60 & ICRP-60 & ICRP-60 & $\begin{array}{l}\text { ICRP-60 methodology based DCFs } \\
\text { (external from FGR-12 and } \\
\text { internal from ICRP 1996) }\end{array}$ \\
\hline Cut-off half-life (180 d or $30 \mathrm{~d}$ ) & $30 \mathrm{~d}$ & $30 \mathrm{~d}$ & $30 \mathrm{~d}$ & $30 \mathrm{~d}$ & RESRAD default \\
\hline Number of points $(32,64,128,256,512,1024)$ & 32 & 32 & 32 & 32 & RESRAD default \\
\hline Linear spacing/log spacing & Log Spacing & Log Spacing & Log Spacing & Log Spacing & RESRAD default \\
\hline Maximum no. of points for dose & 17 & 17 & 17 & 17 & RESRAD default \\
\hline Maximum no. of points for risk & 1 & 1 & 1 & 1 & $\begin{array}{l}\text { Using a smaller integration point } \\
\text { will shorten the calculation time }\end{array}$ \\
\hline Use line draw character (yes/no) & Yes & Yes & Yes & Yes & RESRAD default \\
\hline Find peak pathway dose (yes/no) & Yes & Yes & Yes & Yes & \\
\hline Save all files after each run (yes/no) & Yes & Yes & Yes & Yes & \\
\hline Time integrated probabilistic risk (yes/no) & No & No & No & No & $\begin{array}{l}\text { Dose-to-source ratio (DSR) is } \\
\text { calculated }\end{array}$ \\
\hline \multicolumn{6}{|l|}{ Calculation Parameters } \\
\hline Basic radiation dose limit (mrem/year) & 25 & 25 & 25 & 25 & Not used in DSR calculation \\
\hline Times for calculation (years) & $\begin{array}{l}1,3,10,30,100 \\
300,1,000\end{array}$ & $\begin{array}{c}1,2,3,4,5,6,7,8 \\
970\end{array}$ & $100,300,1,000$ & $100,300,1,000$ & $\begin{array}{l}\text { Up to the time horizon for dose } \\
\text { calculation }\end{array}$ \\
\hline \multicolumn{6}{|l|}{ Source } \\
\hline Nuclide concentration (pCi/g) & 1 & 1 & 1 & 1 & Dose-source ratios calculated \\
\hline \multicolumn{6}{|l|}{ Transport Factors } \\
\hline Distribution coefficient for all zones $\left(\mathrm{cm}^{3} / \mathrm{g}\right)$ & Table B.2 & Table B.2 & Table B.2 & Table B.2 & Site-specific soil type \\
\hline Number of unsaturated zones & 1 & 1 & 1 & 1 & RESRAD default \\
\hline Time since placement of material (y) & 0 & 0 & 0 & 0 & RESRAD default \\
\hline Groundwater concentration $(\mathrm{pCi} / \mathrm{L})$ & 0 & 0 & 0 & 0 & RESRAD default \\
\hline Leach rate $(1 / y)$ & 0 & 0 & 0 & 0 & RESRAD default \\
\hline Solubility limit (mol/L) & 0 & 0 & 0 & 0 & RESRAD default \\
\hline Use plant/soil ration (check box) & No & No & No. & No & RESRAD default \\
\hline
\end{tabular}


TABLE B.1 (Cont.)

\begin{tabular}{|c|c|c|c|c|c|}
\hline \multirow[b]{2}{*}{ Input Parameters } & \multicolumn{2}{|c|}{ Current Use Scenarios } & \multicolumn{2}{|c|}{ Future Use Scenarios } & \multirow[b]{2}{*}{ Comments } \\
\hline & Industrial Worker & Offsite Resident ${ }^{\mathrm{b}}$ & Resident Farmer $^{\mathrm{b}}$ & Recreationist $\mathrm{t}^{\mathrm{c}}$ & \\
\hline \multicolumn{6}{|l|}{ Transfer Factors } \\
\hline $\begin{array}{l}\text { Plant transfer factor (wet-plant weight } \\
\text { concentration, } \mathrm{pCi} / \mathrm{g} / \text { dry soil weight } \\
\text { concentration, } \mathrm{pCi} / \mathrm{g} \text { ) }\end{array}$ & $\mathrm{NR}^{\mathrm{d}}$ & Table B.2 & Table B.2 & $\mathrm{NR}$ & RESRAD default \\
\hline $\begin{array}{l}\text { Meat transfer factor (concentration in meat, } \\
\mathrm{pCi} / \mathrm{g} / \text { rate of intake, } \mathrm{pCi} / \mathrm{d} \text { ) }\end{array}$ & NR & Table B.2 & Table B.2 & Table B.2 & RESRAD default \\
\hline $\begin{array}{l}\text { Milk transfer factor (concentration in milk, } \\
\mathrm{pCi} / \mathrm{L} / \text { rate of intake, } \mathrm{pCi} / \mathrm{d})\end{array}$ & NR & Table B.2 & Table B.2 & NR & RESRAD default \\
\hline $\begin{array}{l}\text { Fish transfer factor (concentration in fish, } \\
\mathrm{pCi} / \mathrm{Kg} / \text { concentration in water, } \mathrm{pCi} / \mathrm{L} \text { ) }\end{array}$ & NR & Table B.3 & Table B.3 & Table B.3 & RESRAD default \\
\hline $\begin{array}{l}\text { Crustacea transfer factor (concentration in } \\
\text { crustacea, } \mathrm{pCi} / \mathrm{kg} / \text { concentration in water, } \\
\mathrm{pCi} / \mathrm{L} \text { ) }\end{array}$ & NR & Table B.3 & Table B.3 & Table B.3 & RESRAD default \\
\hline \multicolumn{6}{|l|}{ Contaminated Zone Parameters } \\
\hline Area of contaminated zone $\left(\mathrm{m}^{2}\right)$ & 10,000 & 10,000 & 10,000 & 10,000 & Matton 2011; Kamboj 2011 \\
\hline Thickness of contaminated zone (m) & 1 & 1 & 1 & 1 & Matton 2011; Kamboj 2011 \\
\hline Length parallel to aquifer flow (m) & 100 & 100 & 100 & 100 & RESRAD default \\
\hline $\begin{array}{l}\text { Does the initial contamination penetrate the } \\
\text { water table? }\end{array}$ & No & No & No & No & RESRAD default \\
\hline \multicolumn{6}{|l|}{ Cover and Contaminated Zone Hydrological } \\
\hline Cover depth (m) & 0 & 0 & 0 & 0 & No cover layer assumed \\
\hline Density of cover material $\left(\mathrm{g} / \mathrm{cm}^{3}\right)$ & NR & NR & NR & NR & $\begin{array}{l}\text { Not required when cover depth } \\
\text { equals zero }\end{array}$ \\
\hline Cover erosion rate $(\mathrm{m} / \mathrm{y})$ & NR & NR & NR & NR & $\begin{array}{l}\text { Not required when cover depth } \\
\text { equals zero }\end{array}$ \\
\hline Density of primary contaminated zone $\left(\mathrm{g} / \mathrm{cm}^{3}\right)$ & 1.76 & 1.76 & 1.76 & 1.76 & Site-specific (ANL 1996) \\
\hline Contaminated zone erosion rate $(\mathrm{m} / \mathrm{y})$ & $1.65 \mathrm{E}-4$ & $1.65 \mathrm{E}-4^{\mathrm{e}}$ & $1.65 \mathrm{E}-4$ & $1.65 \mathrm{E}-4$ & $\begin{array}{l}\text { To match the soil erosion of } \\
1 \text { ton/acre/yr at Illinois } \\
\text { (NRCS 2010) }\end{array}$ \\
\hline Contaminated zone total porosity & 0.3 & 0.3 & 0.3 & 0.3 & Site-specific (ANL 1996) \\
\hline Contaminated zone field capacity & 0.2 & 0.2 & 0.2 & 0.2 & RESRAD default \\
\hline
\end{tabular}


TABLE B.1 (Cont.)

\begin{tabular}{|c|c|c|c|c|c|}
\hline \multirow[b]{2}{*}{ Input Parameters } & \multicolumn{2}{|c|}{ Current Use Scenarios } & \multicolumn{2}{|c|}{ Future Use Scenarios } & \multirow[b]{2}{*}{ Comments } \\
\hline & Industrial Worker & Offsite Resident $\mathrm{t}^{\mathrm{b}}$ & Resident Farmer $^{\mathrm{b}}$ & Recreationist ${ }^{\mathrm{c}}$ & \\
\hline Contaminated zone hydraulic conductivity (m/y) & 0.32 & 0.32 & 0.32 & 0.32 & Site-specific (Quinn 2010) \\
\hline Contaminated zone $\mathrm{b}$ parameter & 10.4 & 10.4 & 10.4 & 10.4 & $\begin{array}{l}\text { Site-specific soil type (silty clay) } \\
\text { value from Yu et al. } 1993^{\mathrm{f}}\end{array}$ \\
\hline Humidity in air $\left(\mathrm{g} / \mathrm{m}^{3}\right)$ & 8 & 8 & 8 & 8 & Used only for $\mathrm{H}-3$ \\
\hline Evapotranspiration coefficient & 0.65 & 0.65 & 0.65 & 0.65 & Site-specific \\
\hline Wind speed $(\mathrm{m} / \mathrm{s})$ & 4.6 & NR & 4.6 & 4.6 & Site-specific value for Chicago \\
\hline Precipitation rate $(\mathrm{m} / \mathrm{y})$ & 0.9177 & 0.9177 & 0.9177 & 0.9177 & $\begin{array}{l}\text { Site-specific value from Golchert } \\
\text { et al. } 1010^{\mathrm{b}}\end{array}$ \\
\hline Irrigation rate $(\mathrm{m} / \mathrm{y})$ & 0.0 & 0.1 & 0.1 & 0.0 & Site-specific (ANL 1996) \\
\hline Irrigation mode (overhead/ditch) & NR & NR & Overhead & NR & RESRAD default \\
\hline Runoff coefficient & 0.2 & 0.2 & 0.2 & 0.2 & $\begin{array}{l}\text { Site-specific for woodland (Yu et } \\
\text { al. 2001, page E-7) }\end{array}$ \\
\hline Watershed area for nearby stream or pond $\left(\mathrm{m}^{2}\right)$ & $1,000,000$ & NR & $1,000,000$ & $1,000,000$ & RESRAD default \\
\hline Accuracy for water/soil computation & 0.001 & NR & 0.001 & 0.001 & RESRAD default \\
\hline \multicolumn{6}{|l|}{ Saturated Zone Hydrological Data } \\
\hline Density of saturated zone $\left(\mathrm{g} / \mathrm{cm}^{3}\right)$ & NR & 1.76 & 1.76 & 1.76 & Site-specific (ANL 1996) \\
\hline Saturated zone effective porosity & NR & 0.14 & 0.14 & 0.14 & Site-specific \\
\hline Saturated zone total porosity & NR & 0.3 & 0.3 & 0.3 & Site-specific (ANL 1996) \\
\hline Saturated zone field capacity & NR & 0.14 & 0.14 & 0.14 & Site-specific \\
\hline Saturated zone hydraulic conductivity $(\mathrm{m} / \mathrm{y})$ & NR & 947 & 947 & 947 & Site-specific (ANL 1996) \\
\hline Saturated zone b parameter & NR & 4.38 & 4.38 & 4.38 & Site-specific soil type \\
\hline Saturated zone hydraulic gradient & NR & .015 & .015 & .015 & Site-specific (Patton et al. 1990) \\
\hline Water table drop rate $(\mathrm{m} / \mathrm{y})$ & NR & NR & 0.001 & 0.001 & RESRAD default \\
\hline Well pump intake depth ( $\mathrm{m}$ below water table) & NR & NR & 5 & 5 & Site-specific \\
\hline $\begin{array}{l}\text { Model for water transportation } \\
\text { (nondispersion/mass-balance) }\end{array}$ & NR & NR & Nondispersion & Nondispersion & RESRAD default \\
\hline Well pumping rate $\left(\mathrm{m}^{3} / \mathrm{y}\right)$ & NR & NR & 250 & 250 & RESRAD default \\
\hline \multicolumn{6}{|l|}{ Unsaturated Zone Parameters } \\
\hline Thickness of unsaturated zone (m) & NR & 13.9 & 13.9 & 13.9 & $\begin{array}{l}\text { Site-specific }(48 \mathrm{ft}-1 \mathrm{~m})(\text { Quinn } \\
\text { 2010) or scenario-specific }\end{array}$ \\
\hline Density of unsaturated zone $\left(\mathrm{g} / \mathrm{cm}^{3}\right)$ & NR & 1.76 & 1.76 & 1.76 & Site-specific (ANL 1996) \\
\hline Unsaturated zone effective porosity & NR & 0.2 & 0.2 & 0.2 & Site-specific (ANL 1996) \\
\hline
\end{tabular}


TABLE B.1 (Cont.)

\begin{tabular}{|c|c|c|c|c|c|}
\hline \multirow[b]{2}{*}{ Input Parameters } & \multicolumn{2}{|c|}{ Current Use Scenarios } & \multicolumn{2}{|c|}{ Future Use Scenarios } & \multirow[b]{2}{*}{ Comments } \\
\hline & Industrial Worker & Offsite Resident ${ }^{\mathrm{b}}$ & Resident Farmer ${ }^{\mathrm{b}}$ & Recreationist ${ }^{\mathrm{c}}$ & \\
\hline Unsaturated zone total porosity & NR & 0.3 & 0.3 & 0.3 & Site-specific (ANL 1996) \\
\hline Unsaturated zone field capacity & NR & 0.2 & 0.2 & 0.2 & RESRAD default \\
\hline Unsaturated zone hydraulic conductivity (m/y) & NR & 0.32 & 0.32 & 0.32 & Site-specific (Quinn 2010) \\
\hline Unsaturated zone $b$ parameter & NR & 10.4 & 10.4 & 10.4 & $\begin{array}{l}\text { Site-specific soil type (silty clay) } \\
\text { value from Yu et al. } 1993\end{array}$ \\
\hline \multicolumn{6}{|l|}{$\begin{array}{l}\text { Occupancy, Inhalation, and External Gamma } \\
\text { Parameters }\end{array}$} \\
\hline Inhalation rate $\left(\mathrm{m}^{3} / \mathrm{y}\right)$ & 11,400 & 8,400 & 8,400 & 14,000 & Scenario-specific \\
\hline Mass loading for inhalation $\left(\mathrm{g} / \mathrm{m}^{3}\right)$ & $1 \mathrm{E}-4$ & $1 \mathrm{E}-4$ & $1 \mathrm{E}-4$ & $1 \mathrm{E}-4$ & RESRAD default \\
\hline Exposure duration (y) & 25 & 30 & 30 & 30 & Not used in DSR calculations \\
\hline Indoor dust filtration factor & 0.4 & 0.4 & 0.4 & 0.4 & RESRAD default \\
\hline External gamma shielding factor & NR & 0.7 & 0.7 & NR & RESRAD default \\
\hline Indoor time fraction & $0 / 0.2283$ & NR & 0.6833 & 0 & Scenario-specific \\
\hline Outdoor time fraction & $0.2283 / 0$ & NR & 0.0833 & 0.0384 & Scenario-specific \\
\hline $\begin{array}{l}\text { Shape of contaminated zone } \\
\text { (circular/noncircular) }\end{array}$ & Circular & Circular & Circular & Circular & RESRAD default \\
\hline \multicolumn{6}{|l|}{ Ingestion Pathway Dietary Data } \\
\hline Fruit, vegetable and grain consumption $(\mathrm{kg} / \mathrm{y})$ & NR & 160 & 160 & NR & RESRAD default \\
\hline Leafy vegetable consumption (kg/y) & NR & 14 & 14 & NR & RESRAD default \\
\hline Milk consumption $(\mathrm{L} / \mathrm{y})$ & NR & 92 & 92 & NR & RESRAD default \\
\hline Meat and poultry consumption $(\mathrm{kg} / \mathrm{y})$ & NR & 63 & 63 & 19 & $\begin{array}{l}\text { RESRAD default and scenario- } \\
\text { specific for recreational use }\end{array}$ \\
\hline Fish consumption $(\mathrm{kg} / \mathrm{y})$ & NR & 5.4 & 5.4 & 12 & $\begin{array}{l}\text { RESRAD default and scenario- } \\
\text { specific for recreational use }\end{array}$ \\
\hline Other sea food consumption $(\mathrm{kg} / \mathrm{y})$ & NR & 0.9 & 0.9 & NR & RESRAD default \\
\hline Soil ingestion $(\mathrm{g} / \mathrm{y})$ & 36.5 & 36.5 & 36.5 & 36.5 & RESRAD default \\
\hline Drinking water intake $(\mathrm{L} / \mathrm{y})$ & NR & 510 & 510 & $1.4 \mathrm{~L} / \mathrm{d}^{\mathrm{g}} 14=19.6 \mathrm{~L}$ & $\begin{array}{l}\text { RESRAD default and scenario- } \\
\text { specific for recreational use }\end{array}$ \\
\hline Drinking water contaminated fraction & NR & 0 & 1 & 1 & $\begin{array}{l}\text { RESRAD default and for offsite } \\
\text { receptor scenario, water is not } \\
\text { contaminated }\end{array}$ \\
\hline Household water contaminated fraction & 1 & 1. & 1 & NR & RESRAD default \\
\hline
\end{tabular}


TABLE B.1 (Cont.)

Current Use Scenarios

Future Use Scenarios

Input Parameters

Livestock water contaminated fraction Irrigation water contaminated fraction

Aquatic food contaminated fraction

Plant food contaminated fraction

Meat contaminated fraction

Milk contaminated fraction

Ingestion Pathway, Nondietary Data

Industrial Worker

Offsite Resident ${ }^{\mathrm{b}}$

Resident Farmer ${ }^{\mathrm{b}}$

Recreationist

Comments

$\begin{array}{ll}\text { NR } & 1 \\ \text { NR } & \\ \text { NR } & 0 \\ \text { NR } & -1 \\ \text { NR } & -1 \\ \text { NR } & -1\end{array}$

1
1
0.5
-1
-1
-1

1
1
0.5
-1
-1
-1

Livestock fodder intake for meat $(\mathrm{kg} / \mathrm{d})$

Livestock fodder intake for milk $(\mathrm{kg} / \mathrm{d})$

Livestock water intake for meat $(\mathrm{L} / \mathrm{d})$

Livestock water intake for milk (L/d)

Livestock intake of soil for meat $(\mathrm{kg} / \mathrm{d})$

Livestock intake of soil for milk $(\mathrm{kg} / \mathrm{d})$

Mass loading for foliar deposition $\left(\mathrm{g} / \mathrm{m}^{3}\right)$

Depth of soil mixing layer (m)

Depth of roots (m)

Drinking water fraction from groundwater

source

Household water fraction from groundwater Source

Livestock water fraction from groundwater source

Irrigation water fraction from groundwater source

NR

NR

NR

NR

NR

NR

NR

NR

0.15

NR

NR

NR

NR

NR

50

160

NR

NR

NR

0.15

0.9

0

0

0

NR

0

0.7

68

55

50

160

0.5

0.5
0.0001

0.15

0.9

1

1

1

Wet weight crop yield for non-leafy vegetables $\left(\mathrm{kg} / \mathrm{m}^{2}\right)$
NR
RESRAD default

RESRAD default

Distribution from NRC 2000

Calculated by RESRAD from area factor

Scenario specific

Calculated by RESRAD from area factor

RESRAD default or scenariospecific

RESRAD default

Or scenario-specific

RESRAD default

RESRAD default

RESRAD default

RESRAD default

RESRAD default

RESRAD default

Scenario-specific

Scenario-specific

RESRAD default for resident farmer scenario and 0 for recreational use scenario because surface water is

used by livestock

RESRAD default 
TABLE B.1 (Cont.)

\begin{tabular}{|c|c|c|c|c|c|}
\hline \multirow[b]{2}{*}{ Input Parameters } & \multicolumn{2}{|c|}{ Current Use Scenarios } & \multicolumn{2}{|c|}{ Future Use Scenarios } & \multirow[b]{2}{*}{ Comments } \\
\hline & Industrial Worker & Offsite Resident $\mathrm{t}^{\mathrm{b}}$ & Resident Farmer $^{\mathrm{b}}$ & Recreationist $^{\mathrm{c}}$ & \\
\hline $\begin{array}{l}\text { Length of growing season for non-leafy } \\
\text { vegetables }(y)\end{array}$ & NR & 0.17 & 0.17 & NR & RESRAD default \\
\hline Translocation factor for non-leafy vegetables & NR & 0.1 & 0.1 & NR & RESRAD default \\
\hline Weathering removal constant $(1 / \mathrm{y})$ & NR & 20 & 20 & 20 & Distribution from NRC 2000 \\
\hline $\begin{array}{l}\text { Wet foliar interception fraction for non-leafy } \\
\text { vegetables }\end{array}$ & NR & 0.25 & 0.25 & NR & RESRAD default \\
\hline $\begin{array}{l}\text { Dry foliar interception fraction or non-leafy } \\
\text { vegetables }\end{array}$ & NR & 0.25 & 0.25 & NR & RESRAD default \\
\hline $\begin{array}{l}\text { Wet weight crop yield for leafy vegetables } \\
\left(\mathrm{kg} / \mathrm{m}^{2}\right)\end{array}$ & NR & 1.5 & 1.5 & NR & RESRAD default \\
\hline $\begin{array}{l}\text { Length of growing season for leafy vegetables } \\
\text { (y) }\end{array}$ & NR & 0.25 & 0.25 & NR & RESRAD default \\
\hline Translocation factor for leafy vegetables & NR & 1 & 1 & NR & RESRAD default \\
\hline $\begin{array}{l}\text { Wet foliar interception fraction for leafy } \\
\text { vegetables }\end{array}$ & NR & 0.25 & 0.25 & NR & RESRAD default \\
\hline $\begin{array}{l}\text { Dry foliar interception fraction for leafy } \\
\text { vegetables }\end{array}$ & NR & 0.25 & 0.25 & NR & RESRAD default \\
\hline Wet weight crop yield for fodder $\left(\mathrm{kg} / \mathrm{m}^{2}\right)$ & NR & NR & 1.1 & 1.1 & RESRAD default \\
\hline Length of growing season for fodder $(\mathrm{y})$ & NR & NR & 0.08 & 0.08 & RESRAD default \\
\hline Translocation factor for fodder & NR & NR & 1 & 1 & RESRAD default \\
\hline Wet foliar interception fraction for fodder & NR & NR & 0.25 & 0.25 & RESRAD default \\
\hline Dry foliar interception fraction for fodder & NR & NR & 0.25 & 0.25 & RESRAD default \\
\hline \multicolumn{6}{|l|}{ Storage-Times-Before-Use Data } \\
\hline $\begin{array}{l}\text { Storage time for fruits, non-leafy vegetables and } \\
\text { grain (d) }\end{array}$ & NR & 14 & 14 & NR & RESRAD default \\
\hline Storage time for leafy vegetables (d) & NR & 1 & 1 & NR & RESRAD default \\
\hline Storage time for milk (d) & NR & 1 & 1 & NR & RESRAD default \\
\hline Storage time for meat (d) & NR & NR & 20 & 182.5 & $\begin{array}{l}\text { RESRAD default for resident farmer } \\
\text { scenario and scenario-specific for } \\
\text { recreational use }\end{array}$ \\
\hline Storage time for fish (d) & NR & 7 & 7 & 0 & $\begin{array}{l}\text { RESRAD default and scenario- } \\
\text { specific for recreational use }\end{array}$ \\
\hline
\end{tabular}


TABLE B.1 (Cont.)

Current Use Scenarios

Future Use Scenarios

Input Parameters

Industrial Worker

Offsite Resident ${ }^{\mathrm{b}}$

Resident Farmer ${ }^{\mathrm{b}}$

Recreationist

Comments

Storage time for crustacea and mollusks (d)

Storage time for well water (d)

Storage time for surface water (d)

Storage time for livestock fodder (d)

\section{Radon Data ${ }^{\mathrm{g}}$}

Cover volumetric water content

Cover radon diffusion coefficient

Total porosity of the house or building

foundation

Volumetric water content of the foundation

Diffusion coefficient for radon gas in foundation

material $\left(\mathrm{m}^{2} / \mathrm{s}\right)$

Diffusion coefficient for radon gas in

contaminated zone soil $\left(\mathrm{m}^{2} / \mathrm{s}\right)$

\section{NR}

NR

NR

NR

Emanating power of radon-222

Radon vertical dimension of mixing $(\mathrm{m})$

Average building air exchange rate $\left(\mathrm{h}^{-1}\right)$

Height of building (room) (m)

Building indoor area factor

Bulk density of house or building foundation

$\left(\mathrm{g} / \mathrm{cm}^{3}\right)$

Thickness of house or building foundation (m)

Building depth below ground surface (m)

\begin{tabular}{|c|c|c|c|c|}
\hline NR & NR & NR & NR & No cover \\
\hline NR & NR & NR & NR & No cover \\
\hline NR & NR & NR & NR & No cover \\
\hline 0.1 & 0.1 & 0.1 & 0.1 & RESRAD default \\
\hline 0.03 & 0.03 & 0.03 & 0.03 & RESRAD default \\
\hline $3 \mathrm{E}-07$ & $3 \mathrm{E}-07$ & $3 \mathrm{E}-07$ & $3 \mathrm{E}-07$ & RESRAD default \\
\hline $2 \mathrm{E}-06$ & 2E-06 & 2E-06 & 2E-06 & $\begin{array}{l}\text { RESRAD default (the value is } \\
\text { representative of silty clay soil } \\
\text { type) }\end{array}$ \\
\hline 0.28 & 0.28 & 0.28 & 0.28 & Clay soil type (Yu et al. 1993) \\
\hline 2.0 & 2.0 & 2.0 & 2.0 & RESRAD default \\
\hline 0.5 & 0.5 & 0.5 & 0.5 & RESRAD default \\
\hline 2.5 & 2.5 & 2.5 & 2.5 & RESRAD default \\
\hline 0 & 0 & 0 & 0 & RESRAD default \\
\hline 2.4 & 2.4 & 2.4 & 2.4 & RESRAD default \\
\hline 0.15 & 0.15 & 0.15 & 0.15 & RESRAD default \\
\hline-1 & -1 & -1 & -1 & RESRAD default \\
\hline
\end{tabular}

RESRAD default and scenariospecific for recreational use

RESRAD default for resident farmer scenario and not required for recreational use scenario

RESRAD default for resident farmer scenario and 0 for recreational use scenario

RESRAD default for resident farmer scenario and scenario-specific for recreational use scenario 
TABLE B.1 (Cont.)

\begin{tabular}{|c|c|c|c|c|c|}
\hline \multirow[b]{2}{*}{ Input Parameters } & \multicolumn{2}{|c|}{ Current Use Scenarios } & \multicolumn{2}{|c|}{ Future Use Scenarios } & \multirow[b]{2}{*}{ Comments } \\
\hline & Industrial Worker & Offsite Resident ${ }^{\mathrm{b}}$ & Resident Farmer ${ }^{\mathrm{b}}$ & Recreationist $^{\mathrm{c}}$ & \\
\hline \multicolumn{6}{|l|}{ Carbon-14 Data ${ }^{\mathrm{g}}$} \\
\hline C-12 concentration in local water $\left(\mathrm{g} / \mathrm{cm}^{3}\right)$ & $2 \mathrm{E}-5$ & $2 \mathrm{E}-5$ & $2 \mathrm{E}-5$ & $2 \mathrm{E}-5$ & RESRAD default \\
\hline $\mathrm{C}-12$ concentration in contaminated soil $(\mathrm{g} / \mathrm{g})$ & 0.03 & 0.03 & 0.03 & 0.03 & RESRAD default \\
\hline $\begin{array}{l}\text { Fraction of vegetation carbon absorbed from } \\
\text { soil }\end{array}$ & 0.02 & 0.02 & 0.02 & 0.02 & RESRAD default \\
\hline Fraction of vegetation carbon absorbed from air & 0.98 & 0.98 & 0.98 & 0.98 & RESRAD default \\
\hline Thickness of evasion layer of C-14 in soil (m) & 0.3 & 0.3 & 0.3 & 0.3 & RESRAD default \\
\hline $\mathrm{C}-14$ evasion flux rate from soil $\left(\mathrm{sec}^{-1}\right)$ & $7 \mathrm{E}-7$ & $7 \mathrm{E}-7$ & $7 \mathrm{E}-7$ & $7 \mathrm{E}-7$ & RESRAD default \\
\hline C-12 evasion flux rate from soil $\left(\mathrm{sec}^{-1}\right)$ & $1 \mathrm{E}-10$ & $1 \mathrm{E}-10$ & $1 \mathrm{E}-10$ & $1 \mathrm{E}-10$ & RESRAD default \\
\hline Grain fraction in beef cattle feed & 0.8 & 0.8 & 0.8 & 0.8 & RESRAD default \\
\hline Grain fraction in milk cow feed & 0.2 & 0.2 & 0.2 & 0.2 & RESRAD default \\
\hline
\end{tabular}

a RESRAD-OFFSITE code is used for analyzing offsite resident scenario, and RESRAD (onsite) code is used for other scenarios. Extra parameters required for RESRADOFFSITE code are listed in Table B-4.

b For offsite resident and residential farmer scenarios, the parameter values for food consumption are RESRAD defaults. Parameter values for inhalation rate, indoor and outdoor time fractions, are scenario-specific. The parameter values for livestock fodder intake for meat, livestock water intake for meat, livestock intake of soil, plant food contaminated fraction, and meat contaminated fractions are code defaults and are calculated based on the contaminated area. The parameter values for storage time of meat, fish, surface water, and livestock fodder are RESRAD defaults.

c For the recreational use scenario, it is assumed that the individual would be involved in moderate activity onsite; therefore, an inhalation rate of moderate activity $\left(1.6 \mathrm{~m}^{3} / \mathrm{h}\right)$ is assumed (EPA 1997). The parameter value for meat and poultry consumption is based on the assumption that a recreationist kills a deer and brings the catch home and uses its meat over a year; the value is based on the game consumption statistics among households that hunt. For fish consumption, it is assumed that a recreationist catches a fish and consumes it the same day; value is based on the home-caught fish consumption statistics among households that fish. The parameter values for indoor and outdoor time fractions are from scenario description. All the meat consumed is assumed to be contaminated. For the storage time of meat, since the meat is consumed over a year, an average storage time of 182.5 days is assumed. For the storage time of fish, since the fish is consumed on the same day, a storage time of zero days is assumed. Since the game animal uses fresh forage and surface water, a storage time of zero days is assumed for both parameters.

d NR - value is not required for this scenario.

e Cover and management are changed to 0.0506 to get site-specific erosion rate in RESRAD-OFFSITE areas.

f It is assumed that the soil type is silty clay at the Argonne site, and values representative of silty clay soil are used.

$\mathrm{g}$ All the parameters for radon (except radon emanation rate) and C-14 are kept at code default values. 
TABLE B.2 Parameter Values Used in the Deterministic Analysis for $K_{d}$, Plant, Meat, and Milk Transfer Factors

\begin{tabular}{|c|c|c|c|c|c|}
\hline \multirow[b]{2}{*}{ Element } & \multicolumn{2}{|c|}{$\mathrm{K}_{\mathrm{d}}\left(\mathrm{cm}^{3} / \mathrm{g}\right)$} & \multirow[b]{2}{*}{$\begin{array}{c}\text { Plant Transfer } \\
\text { Factor } \\
(\mathrm{pCi} / \mathrm{kg}) /(\mathrm{pCi} / \mathrm{kg})\end{array}$} & \multirow[b]{2}{*}{$\begin{array}{c}\text { Meat Transfer } \\
\text { Factor } \\
(\mathrm{pCi} / \mathrm{kg}) /(\mathrm{pCi} / \mathrm{d})\end{array}$} & \multirow[b]{2}{*}{$\begin{array}{l}\text { Milk Transfer } \\
\text { Factor } \\
(\mathrm{pCi} / \mathrm{L}) /(\mathrm{pCi} / \mathrm{d})\end{array}$} \\
\hline & $\begin{array}{l}\text { Saturated } \\
\text { Zone }^{\mathrm{a}}\end{array}$ & $\begin{array}{l}\text { Contaminated and } \\
\text { Unsaturated Zone }\end{array}$ & & & \\
\hline Ac & 450 & 2,400 & $2.5 \times 10^{-3}$ & $2.0 \times 10^{-5}$ & $2.0 \times 10^{-5}$ \\
\hline $\mathrm{Am}$ & 1,900 & 8,400 & $1.0 \times 10^{-3}$ & $5.0 \times 10^{-5}$ & $2.0 \times 10^{-6}$ \\
\hline $\mathrm{Ba}$ & 560 & 560 & $5.0 \times 10^{-3}$ & $2.0 \times 10^{-4}$ & $5.0 \times 10^{-4}$ \\
\hline $\mathrm{C}$ & 5 & 1 & $7.0 \times 10^{-1}$ & $3.1 \times 10^{-2}$ & $1.2 \times 10^{-2}$ \\
\hline Cs & 280 & 1,900 & $4.0 \times 10^{-2}$ & $3.0 \times 10^{-2}$ & $8.0 \times 10^{-3}$ \\
\hline $\mathrm{Np}$ & 5 & 55 & $2.0 \times 10^{-2}$ & $1.0 \times 10^{-3}$ & $5.0 \times 10^{-6}$ \\
\hline $\mathrm{Pa}$ & 550 & 2,700 & $1.0 \times 10^{-2}$ & $5.0 \times 10^{-3}$ & $5.0 \times 10^{-6}$ \\
\hline $\mathrm{Pb}$ & 270 & 550 & $1.0 \times 10^{-2}$ & $8.0 \times 10^{-4}$ & $3.0 \times 10^{-4}$ \\
\hline Po & 150 & 3,000 & $1.0 \times 10^{-3}$ & $5.0 \times 10^{-3}$ & $3.4 \times 10^{-4}$ \\
\hline $\mathrm{Pu}$ & 550 & 5,100 & $1.0 \times 10^{-3}$ & $1.0 \times 10^{-4}$ & $1.0 \times 10^{-6}$ \\
\hline $\mathrm{Ra}$ & 500 & 9,100 & $4.0 \times 10^{-2}$ & $1.0 \times 10^{-3}$ & $1.0 \times 10^{-3}$ \\
\hline $\mathrm{Sr}$ & 15 & 110 & $3.0 \times 10^{-1}$ & $8.0 \times 10^{-3}$ & $2.0 \times 10^{-3}$ \\
\hline Tc & 0.1 & 1 & $5.0 \times 10^{0}$ & $1.0 \times 10^{-4}$ & $1.0 \times 10^{-3}$ \\
\hline Th & 3,200 & 5,800 & $1.0 \times 10^{-3}$ & $1.0 \times 10^{-4}$ & $5.0 \times 10^{-6}$ \\
\hline $\mathrm{U}$ & 35 & 1,600 & $2.5 \times 10^{-3}$ & $3.4 \times 10^{-4}$ & $6.0 \times 10^{-4}$ \\
\hline
\end{tabular}

a Based on sandy soil type from RESRAD data collection handbook or RESRAD default values for saturated zone.

b Based on clay soil type from RESRAD data collection handbook or RESRAD default values for contaminated and unsaturated zone. 
TABLE B.3 Parameter Value/Distribution Used in the Analysis for the Fish and Crustacea Transfer Factor

\begin{tabular}{ccc}
\hline Element & $\begin{array}{c}\text { Crustacea Transfer Factor } \\
\text { (concentration in crustacea, } \mathrm{pCi} / \mathrm{Kg} / \\
\text { concentration in water, } \mathrm{pCi} / \mathrm{L} \text { ) }\end{array}$ & $\begin{array}{c}\text { Fish Transfer Factor } \\
\text { (concentration in crustacea, } \mathrm{pCi} / \mathrm{Kg} / \\
\text { concentration in water, } \mathrm{pCi} / \mathrm{L} \text { ) }\end{array}$ \\
\hline $\mathrm{Ac}$ & 1,000 & \\
$\mathrm{Am}$ & 1,000 & 15 \\
$\mathrm{Ba}$ & 200 & 30 \\
$\mathrm{C}$ & 9,100 & 4 \\
$\mathrm{Cs}$ & 100 & 50,000 \\
$\mathrm{~Np}$ & 400 & 2,000 \\
$\mathrm{~Pa}$ & 110 & 30 \\
$\mathrm{~Pb}$ & 100 & 10 \\
$\mathrm{Po}$ & 20,000 & 300 \\
$\mathrm{Pu}$ & 100 & 100 \\
$\mathrm{Ra}$ & 250 & 30 \\
$\mathrm{Sr}$ & 100 & 50 \\
$\mathrm{Tc}$ & 5 & 60 \\
$\mathrm{Th}$ & 500 & 20 \\
$\mathrm{U}$ & 60 & 100 \\
\hline
\end{tabular}




\section{TABLE B.4 Additional Input Parameters for RESRAD-OFFSITE Analysis}

\begin{tabular}{|c|c|c|}
\hline Parameter & Value & Comment \\
\hline \multicolumn{3}{|l|}{ Site Layout $^{\mathrm{a}}$} \\
\hline $\mathrm{X}$ dimension of primary contamination $(\mathrm{m})$ & 100 & Total contaminated area $=10,000 \mathrm{~m}^{2}$ \\
\hline Y dimension of primary contamination $(\mathrm{m})$ & 100 & Total contaminated area $=10,000 \mathrm{~m}^{2}$ \\
\hline $\begin{array}{l}\text { Smaller X coordinate of the fruit, grain, non-leafy } \\
\text { vegetables plot }(\mathrm{m})\end{array}$ & 434.4 & Closest distance to the site boundary \\
\hline $\begin{array}{l}\text { Larger X coordinate of the fruit, grain, non-leafy } \\
\text { vegetables plot }(\mathrm{m})\end{array}$ & 466 & $\begin{array}{l}\text { Assume the vegetable plot is } 31.6 \mathrm{~m} \times \\
31.6 \mathrm{~m} \text {, with an area of } 1,000 \mathrm{~m}^{2}\end{array}$ \\
\hline $\begin{array}{l}\text { Smaller Y coordinate of the fruit, grain, non-leafy } \\
\text { vegetables plot }(\mathrm{m})\end{array}$ & 434 & Closest distance to the site boundary \\
\hline $\begin{array}{l}\text { Larger Y coordinate of the fruit, grain, non-leafy } \\
\text { vegetables plot }(\mathrm{m})\end{array}$ & 466 & $\begin{array}{l}\text { Assume the vegetable plot is } 31.6 \mathrm{~m} \times \\
31.6 \mathrm{~m} \text {, with an area of } 1,000 \mathrm{~m}^{2}\end{array}$ \\
\hline Smaller X coordinate of the leafy vegetables plot (m) & 434.4 & Closest distance to the site boundary \\
\hline Larger X coordinate of the leafy vegetables plot (m) & 465.6 & $\begin{array}{l}\text { Assume the vegetable plot is } 31.6 \mathrm{~m} \times \\
31.6 \mathrm{~m} \text {, with an area of } 1,000 \mathrm{~m}^{2}\end{array}$ \\
\hline Smaller Y coordinate of the leafy vegetables plot (m) & 468 & $\begin{array}{l}\text { Leafy vegetable plot is assumed to be } \\
\text { next to the nonleafy vegetable plot }\end{array}$ \\
\hline Larger Y coordinate of the leafy vegetables plot (m) & 500 & $\begin{array}{l}\text { Assume the vegetable plot is } 31.6 \mathrm{~m} \times \\
31.6 \mathrm{~m} \text {, with an area of } 1,000 \mathrm{~m}^{2}\end{array}$ \\
\hline $\begin{array}{l}\text { Smaller X coordinate of the pasture, silage growing } \\
\text { area }(\mathrm{m})\end{array}$ & 450 & Close to the vegetable plots \\
\hline $\begin{array}{l}\text { Larger X coordinate of the pasture, silage growing } \\
\text { area }(\mathrm{m})\end{array}$ & 550 & $\begin{array}{l}\text { Assume the livestock feed area is } 100 \mathrm{~m} \\
\times 100 \mathrm{~m} \text {, with an area of } 10,000 \mathrm{~m}^{2}\end{array}$ \\
\hline $\begin{array}{l}\text { Smaller Y coordinate of the pasture, silage growing } \\
\text { area }(\mathrm{m})\end{array}$ & 500 & Close to the vegetable plots \\
\hline $\begin{array}{l}\text { Larger } Y \text { coordinate of the pasture, silage growing } \\
\text { area }(\mathrm{m})\end{array}$ & 600 & $\begin{array}{l}\text { Assume the livestock feed area is } 100 \mathrm{~m} \\
\times 100 \mathrm{~m} \text {, with an area of } 10,000 \mathrm{~m}^{2}\end{array}$ \\
\hline Smaller X coordinate of the grain fields (m) & 500 & Close to the vegetable plots. \\
\hline Larger X coordinate of the grain fields (m) & 600 & $\begin{array}{l}\text { Assume the livestock feed area is } 100 \mathrm{~m} \\
\times 100 \mathrm{~m} \text {, with an area of } 10,000 \mathrm{~m}^{2}\end{array}$ \\
\hline Smaller Y coordinate of the grain fields (m) & 600 & Close to the vegetable plots \\
\hline Larger Y coordinate of the grain fields $(\mathrm{m})$ & 700 & $\begin{array}{l}\text { Assume the livestock feed area is } 100 \mathrm{~m} \\
\times 100 \mathrm{~m} \text {, with an area of } 10,000 \mathrm{~m}^{2}\end{array}$ \\
\hline Smaller X coordinate of the dwelling site $(\mathrm{m})$ & 434.4 & Closest distance to the site boundary \\
\hline Larger $\mathrm{X}$ coordinate of the dwelling site $(\mathrm{m})$ & 465.6 & $\begin{array}{l}\text { The dwelling site is assumed to be } \\
31.6 \mathrm{~m} \times 31.6 \mathrm{~m} \text { and have an area of } \\
1,000 \mathrm{~m}^{2}\end{array}$ \\
\hline Smaller $Y$ coordinate of the dwelling site $(\mathrm{m})$ & 400 & Closest distance to the site boundary \\
\hline Larger Y coordinate of the dwelling site (m) & 432 & $\begin{array}{l}\text { The dwelling site is assumed to be } \\
31.6 \mathrm{~m} \times 31.6 \mathrm{~m} \text { and have an area of } \\
1,000 \mathrm{~m}^{2}\end{array}$ \\
\hline Smaller X coordinate of the surface water body & 526.5 & $\begin{array}{l}\text { The surface water body is assumed to be } \\
\text { close to the dwelling site and the } \\
\text { vegetable and livestock feed areas }\end{array}$ \\
\hline Larger X coordinate of the surface water body & 826.5 & $\begin{array}{l}\text { The surface water body is assumed to be } \\
\text { close to the dwelling site and the } \\
\text { vegetable and livestock feed areas }\end{array}$ \\
\hline
\end{tabular}




\begin{tabular}{|c|c|c|}
\hline Parameter & Value & Comment \\
\hline Smaller Y coordinate of the surface water body & 356.8 & $\begin{array}{l}\text { The surface water body is assumed to be } \\
\text { close to the dwelling site and the } \\
\text { vegetable and livestock feed areas }\end{array}$ \\
\hline Larger Y coordinate of the surface water body & 656.8 & $\begin{array}{l}\text { The surface water body is assumed to be } \\
\text { close to the dwelling site and the } \\
\text { vegetable and livestock feed areas }\end{array}$ \\
\hline
\end{tabular}

\section{Source Release and Deposition Velocity}

Deposition velocity $(\mathrm{m} / \mathrm{s})$

For all radionuclides except I-129 (0.01)

\section{Distribution Coefficients}

Sediment in surface water body $\left(\mathrm{cm}^{3} / \mathrm{g}\right)$

Fruit, grain, nonleafy vegetable fields $\left(\mathrm{cm}^{3} / \mathrm{g}\right)$

Leafy vegetable fields $\left(\mathrm{cm}^{3} / \mathrm{g}\right)$

Pasture, silage growing areas $\left(\mathrm{cm}^{3} / \mathrm{g}\right)$

Livestock feed grain fields $\left(\mathrm{cm}^{3} / \mathrm{g}\right)$

Off-site dwelling site $\left(\mathrm{cm}^{3} / \mathrm{g}\right)$

\section{Transfer Factors}

Fruit, grain, non-leafy vegetable transfer factor Leafy vegetable transfer factor

Pasture and silage transfer factor

Livestock feed grain transfer factor

\section{Storage Time}

Storage time for pasture and silage (d)

Storage time for livestock feed grain (d)

\section{Primary Contamination}

Deposition velocity of dust $(\mathrm{m} / \mathrm{s})$

Rainfall and runoff factor

Slope-length-steepness factor

Cover and management factor

Support practice factor

\section{Contaminated Zone}

Soil erodibility factor of contaminated zone

\section{Clean Cover}

Soil erodibility factor of clean cover

Volumetric water content of clean cover
Table B.2

Table B.2

Table B.2

Table B.2

Table B.2

Table B.2

Table B.2

Table B. 2

Table B.2

Table B.2

$\begin{array}{cl}1 & \text { Default } \\ 45 & \text { Default } \\ & \\ 0.001 & \text { Default } \\ 160 & \text { Default } \\ 0.4 & \text { Default } \\ 0.0506 & \text { Adjusted to match site-specific erosion } \\ & \text { rate } \\ 1 & \text { Default }\end{array}$

$0.4 \quad$ Default

NR No cover

NR No cover

\section{Agriculture/Livestock Feed Growing/Offsite Dwelling Area Parameters}

Fraction of area directly over primary contamination for all fields

Irrigation applied per year for all fields $(\mathrm{m} / \mathrm{y})$

$0 \quad$ Receptor offsite


Parameter

Evapotranspiration coefficient for all fields

Runoff coefficient for all fields

Depth of soil mixing layer or plow layer for all fields (m)

Volumetric water content for all fields

Dry bulk density of soil for all fields $\left(\mathrm{g} / \mathrm{cm}^{3}\right)$

Soil erodibility factor for all fields

Slope-length-steepness factor for all fields

Cover and management factor for all fields

Support practice factor for all fields

\section{Atmospheric Transport}

Release height (m)

Release heat flux (cal/s)

Anemometer height (m)

Ambient temperature (K)

AM atmospheric mixing height (m)

PM atmospheric mixing height (m)

Dispersion model coefficients

Wind speed terrain

Elevation of offsite location relative to ground level at primary contamination for all fields $(\mathrm{m})$

Grid spacing for areal integration (m)

Joint frequency of wind speed and stability class for a 16-sector wind rose

\section{Unsaturated Zone Parameters}

Unsaturated zone longitudinal dispersivity (m)

\section{Saturated Zone Hydrological Data}

Thickness of saturated zone (m)

Saturated zone longitudinal dispersivity to surface water body $(\mathrm{m})$

Saturated zone horizontal lateral dispersivity to surface water body (m)

Disperse vertically

Saturated zone vertical lateral dispersivity to surface water body $(\mathrm{m})$

Depth of aquifer contributing to surface water body (m)

\section{Surface Water Body}

Sediment deliver ratio

Volume of surface water body $\left(\mathrm{m}^{3}\right)$

Value

Comment

Table B.1

Table B.1

Table B.1

\section{$0.3 \quad$ Default}

Table B.1

0.4 Default

0.4 Default

0.0488 Adjusted to match site-specific erosion rate

$1 \quad$ Default

$\begin{array}{cc}1 & \text { Default } \\ 0 & \text { Default } \\ 10 & \text { Default } \\ 285 & \text { Default } \\ 400 & \text { Default } \\ 1,600 & \text { Default } \\ \text { Pasquill- } & \text { Default } \\ \text { Gifford } & \\ \text { Rural } & \text { Default } \\ 0 & \text { Default } \\ 10 & \text { Default } \\ \text { Actual values } & \text { Site-specific } \\ \text { from Chicago } & \\ \text { Midway } & \end{array}$

\section{$0.1 \quad$ Default}

$\begin{array}{cl}18.3 & \text { Site-specific } \\ 10 & \text { Default } \\ 1 & \text { Default } \\ & \\ \text { Check box } & \text { Yes } \\ 0.06 & \text { Default } \\ 10 & \text { Default }\end{array}$




\section{TABLE B.4 (Cont.)}

Parameter

Mean residence time of water in surface water body

(y)

\section{Groundwater Transport Parameters}

Distance from downgradient edge of contamination

to well in the direction parallel to aquifer flow (m)

Distance from downgradient edge of contamination to surface water body in the direction parallel to aquifer flow (m)

Distance from downgradient edge of contamination to well in the direction perpendicular to aquifer flow $(\mathrm{m})$

Distance from downgradient edge of contamination to the right edge of surface water body in the direction perpendicular to aquifer flow (m)

Distance from downgradient edge of contamination to the left edge of the surface water body in the direction perpendicular to aquifer flow (m)

Main subzones in saturated zone

Main subzones in each partially saturated zone

Nuclide-specific retardation in all subzones, longitudinal dispersion in all but the subzone of transformation?

\section{Water Use}

Quantity of water consumed by an individual (L/yr)

Number of household individuals consuming and using water

Quantity of water for use indoors of dwelling per individual $(\mathrm{L} / \mathrm{d})$

Quantity of water for beef cattle (L/d)

Number of beef cattle

Quantity of water for dairy cows (L/d)

Number of dairy cows

Well pumping rate $\left(\mathrm{m}^{3} / \mathrm{yr}\right)$

Ingestion Rates

Drinking water intake from affected area

Fish intake from affected area

Crustacea and mollusks intake from affected area

Fruit, grain, non-leafy vegetables fraction from affected area

Leafy vegetables fraction from affected area

Meat fraction from affected area

Milk fraction from affected area
Value

Comment

$1 \quad$ Default

$\begin{array}{cl}-1 & \text { Site-specific } \\ 256.8 & \text { Site-specific } \\ -424 & \text { Site-specific } \\ & \\ -150 & \text { Default } \\ & \\ 150 & \text { Default } \\ & \\ 1 & \text { Default } \\ 1 & \text { Default } \\ \text { Yes } & \text { Default }\end{array}$

Table B.1 Household water not contaminated 4 Default

225 Household water not contaminated

$50 \quad$ Default

2 Default

160 Default

2 Default

NR RESRAD default

0 Drinking water not contaminated

0.5 Default

0.5 Default

0.5 Default

$0.5 \quad$ Default

$0.5 \quad 50 \%$ of the meat consumed is from contaminated area

$0.5 \quad 50 \%$ of the meat consumed is from contaminated area 


\section{Livestock Intake}

Pasture and silage intake for beef cattle $(\mathrm{kg} / \mathrm{d})$

Grain intake for beef cattle $(\mathrm{kg} / \mathrm{d})$

Soil from pasture and silage intake for beef cattle (kg/d)

Soil from grain intake for beef cattle $(\mathrm{kg} / \mathrm{d})$

Pasture and silage intake for dairy cows $(\mathrm{kg} / \mathrm{d})$

Grain intake for dairy cows $(\mathrm{kg} / \mathrm{d})$

Soil from pasture and silage intake for dairy cows (kg/d)

Soil from grain intake for dairy cows $(\mathrm{kg} / \mathrm{d})$

$\begin{array}{cc}14 & \text { Default } \\ 54 & \text { Default } \\ 0.1 & \text { Default } \\ 0.4 & \text { Default } \\ 44 & \text { Default } \\ 11 & \text { Default } \\ 0.4 & \text { Default } \\ 0.1 & \text { Default }\end{array}$

\section{Livestock Feed Factors (for Pasture and Silage,}

Grain)

Wet weight crop yield $\left(\mathrm{kg} / \mathrm{m}^{2}\right)$

Duration of growing season (yr)

Foliage to food transfer coefficient

Weathering removal constant

Foliar interception factor for irrigation

Foliar interception factor for dust deposition

Root depth (m)

$\begin{array}{cc}1.1,0.7 & \text { Default } \\ 0.08,0.17 & \text { Default } \\ 1,0.1 & \text { Default } \\ 20,20 & \text { Default } \\ 0.25,0.25 & \text { Default } \\ 0.25,0.25 & \text { Default } \\ \text { Table B.1 } & \end{array}$

\section{Occupancy Factors}

Indoor time fraction on primary contamination Outdoor time fraction on primary contamination Indoor time fraction on offsite dwelling site Outdoor time fraction on offsite dwelling site

Time fraction in fruit, grain, and non-leafy vegetable fields

Time fraction in leafy vegetable fields

Time fraction in pasture and silage fields

Time fraction in livestock grain fields

$\begin{array}{cc}0 & \text { Default } \\ 0 & \text { Default } \\ 0.5 & \text { Default } \\ 0.1 & \text { Default } \\ 0.1 & \text { Default } \\ & \\ 0.1 & \text { Default } \\ 0.1 & \text { Default } \\ 0.1 & \text { Default }\end{array}$

a All the areas (dwelling, agricultural areas) are assumed to be less than $350 \mathrm{~m}$ away from the contaminated area. This is a conservative assumption because the distance to the site boundary from the Building 330 area is greater than $350 \mathrm{~m}$. 


\section{REFERENCES}

ANL (Argonne National Laboratory), 1996, Interim Action Documentation Report:

Decontamination and Demolition of the 317 Area South Vaults, Argonne National Laboratory, Argonne Ill., May 31.

Eckerman, K.F., and J.C. Ryman, 1993, External Exposure to Radionuclides in Air, Water, and Soil, Exposure to Dose Coefficients for General Application, Based on the 1987 Federal Radiation Protection Guidance, EPA 402-R-93-076, Federal Guidance Report No. 12, prepared by Oak Ridge National Laboratory, Oak Ridge, Tenn., for U.S. Environmental Protection Agency, Office of Radiation and Indoor Air, Washington, D.C.

EPA (U.S. Environmental Protection Agency), 1997, Exposure Factor Handbook, EPA/600/P-95/002Fa, Office of Research and Development, National Center for Environmental Assessment, Washington, D.C.

Golchert, N.W., T.M. Davis, and L.P. Moos, 2010, Argonne Site Environmental Report for Calendar Year 2009, ANL-10/02, Argonne National Laboratory, Argonne, Ill., September.

ICRP (International Commission on Radiological Protection), 1991, 1990 Recommendations of the International Commission on Radiological Protection, ICRP Publication 60, Ann. ICRP 21(1-3), Pergamon Press, Oxford, England.

ICRP, 1996, Age-Dependent Doses to Members of the Public from Intake of Radionuclides: Part 5, Compilation of Ingestion and Inhalation Coefficients, ICRP Publication 72, Ann. ICRP 26(1), Pergamon Press, Oxford, England.

Kamboj, S., 2011, personal communication between S. Kamboj, Argonne, and R.L. Johnson, Argonne, Subject: "Bldg. 310 RESRAD Analysis Project Status," January 7.

Matton, P.B., 2011, personal communication between P.B. Matton, Argonne, and R.J. Johnson, Argonne, Subject: "Bldg. 330 D\&D Project-RESRAD, DCGLs, Clean-up Parameters," January 14.

NRC (U.S. Nuclear Regulatory Commission), 2000, Development of Probabilistic RESRAD 6.0 and RESRAD-BUILD 3.0 Computer Codes, NUREG/CR-6697 and ANL/EAD/TM-98, prepared by C. Yu et al., Argonne National Laboratory, Argonne, Ill., for U.S. Nuclear Regulatory Commission, Washington, D.C., Nov.

NRCS (Natural Resources Conservation Service), 2010, "Report Cards Are Out - Illinois NRI Gets Gold Star!", news release, May 25.

Patton, T.L., R.H. Pearl, and S.Y. Tsai, 1990, Hydrological Conditions at the 800 Area at Argonne National Laboratory, ANL/EAIS/TM-29, Argonne National Laboratory, Argonne, Ill., August. 
Quinn, J., 2010, personal communication between J. Quinn (Argonne) and S. Kamboj, Argonne, November 12.

Yu, C., et al., 1993, Data Collection Handbook to Support Modeling the Impacts of Radioactive Material in Soil, ANL/EAIS-8, Argonne National Laboratory, Argonne, Ill., April.

Yu, C., et al., 2001, User's Manual for RESRAD Version 6, ANL/EAD-4, Argonne National Laboratory, Argonne, Ill. 


\section{APPENDIX C:}

\section{DETAILED PROBABILISTIC ANALYSIS RESULTS}

Tables C.1-C.8 show the peak DSR percentiles (mrem/yr per pCi/g) for different exposure pathways for the industrial use scenario. RESRAD (onsite) version 6.5 was used in the analysis. For Ba-133, Cs-137, and Sr-90, external exposure was the dominant exposure pathway. For Am-241 and Tc-99, soil ingestion and external exposure were the dominant exposure pathways. For C-14, only inhalation was the dominant exposure pathway. For Pu-238 and $\mathrm{Pu}-239$ soil ingestion and inhalation were two dominant exposure pathways.

\section{TABLE C.1 Peak DSR Percentiles (mrem/yr per pCi/g) for Different Exposure Pathways for Am-241 in Industrial Use Scenario}

\begin{tabular}{rrrrr}
\hline & & & & \\
Percentile & All Pathways & External & $\begin{array}{c}\text { Inhation } \\
\text { w/o Rn }\end{array}$ & Soil Ingestion \\
\hline & & & & \\
$5 \%$ & $1.47 \mathrm{E}-03$ & $8.66 \mathrm{E}-04$ & $1.86 \mathrm{E}-04$ & $2.76 \mathrm{E}-04$ \\
$10 \%$ & $4.06 \mathrm{E}-03$ & $2.54 \mathrm{E}-03$ & $4.46 \mathrm{E}-04$ & $6.60 \mathrm{E}-04$ \\
$15 \%$ & $6.76 \mathrm{E}-03$ & $4.23 \mathrm{E}-03$ & $6.94 \mathrm{E}-04$ & $1.00 \mathrm{E}-03$ \\
$20 \%$ & $9.42 \mathrm{E}-03$ & $5.89 \mathrm{E}-03$ & $9.35 \mathrm{E}-04$ & $1.33 \mathrm{E}-03$ \\
$25 \%$ & $1.11 \mathrm{E}-02$ & $7.57 \mathrm{E}-03$ & $1.14 \mathrm{E}-03$ & $1.65 \mathrm{E}-03$ \\
$30 \%$ & $1.21 \mathrm{E}-02$ & $7.86 \mathrm{E}-03$ & $1.30 \mathrm{E}-03$ & $1.95 \mathrm{E}-03$ \\
$35 \%$ & $1.27 \mathrm{E}-02$ & $8.15 \mathrm{E}-03$ & $1.44 \mathrm{E}-03$ & $2.27 \mathrm{E}-03$ \\
$40 \%$ & $1.33 \mathrm{E}-02$ & $8.43 \mathrm{E}-03$ & $1.57 \mathrm{E}-03$ & $2.53 \mathrm{E}-03$ \\
$45 \%$ & $1.37 \mathrm{E}-02$ & $8.72 \mathrm{E}-03$ & $1.70 \mathrm{E}-03$ & $2.78 \mathrm{E}-03$ \\
$50 \%$ & $1.43 \mathrm{E}-02$ & $9.01 \mathrm{E}-03$ & $1.82 \mathrm{E}-03$ & $3.02 \mathrm{E}-03$ \\
$55 \%$ & $1.47 \mathrm{E}-02$ & $9.20 \mathrm{E}-03$ & $1.97 \mathrm{E}-03$ & $3.26 \mathrm{E}-03$ \\
$60 \%$ & $1.52 \mathrm{E}-02$ & $9.39 \mathrm{E}-03$ & $2.14 \mathrm{E}-03$ & $3.50 \mathrm{E}-03$ \\
$65 \%$ & $1.57 \mathrm{E}-02$ & $9.58 \mathrm{E}-03$ & $2.34 \mathrm{E}-03$ & $3.73 \mathrm{E}-03$ \\
$70 \%$ & $1.62 \mathrm{E}-02$ & $9.76 \mathrm{E}-03$ & $2.53 \mathrm{E}-03$ & $4.00 \mathrm{E}-03$ \\
$75 \%$ & $1.68 \mathrm{E}-02$ & $9.95 \mathrm{E}-03$ & $2.78 \mathrm{E}-03$ & $4.31 \mathrm{E}-03$ \\
$80 \%$ & $1.75 \mathrm{E}-02$ & $1.05 \mathrm{E}-02$ & $3.07 \mathrm{E}-03$ & $4.64 \mathrm{E}-03$ \\
$85 \%$ & $1.84 \mathrm{E}-02$ & $1.10 \mathrm{E}-02$ & $3.46 \mathrm{E}-03$ & $5.04 \mathrm{E}-03$ \\
$90 \%$ & $1.97 \mathrm{E}-02$ & $1.16 \mathrm{E}-02$ & $3.99 \mathrm{E}-03$ & $5.53 \mathrm{E}-03$ \\
$95 \%$ & $2.19 \mathrm{E}-02$ & $1.23 \mathrm{E}-02$ & $4.87 \mathrm{E}-03$ & $6.27 \mathrm{E}-03$ \\
Mean & $1.35 \mathrm{E}-02$ & $8.26 \mathrm{E}-03$ & $2.11 \mathrm{E}-03$ & $3.09 \mathrm{E}-03$ \\
Min & $1.08 \mathrm{E}-04$ & $7.56 \mathrm{E}-05$ & $1.14 \mathrm{E}-06$ & $5.60 \mathrm{E}-06$ \\
Max & $4.08 \mathrm{E}-02$ & $1.71 \mathrm{E}-02$ & $1.65 \mathrm{E}-02$ & $1.15 \mathrm{E}-02$ \\
\hline & & & & \\
\hline
\end{tabular}


TABLE C.2 Peak DSR Percentiles (mrem/yr per pCi/g) for Different Exposure Pathways for Ba-133 in Industrial Use Scenario

\begin{tabular}{rcccc}
\hline & & & & \\
Percentile & All Pathways & External & $\begin{array}{c}\text { Inhalation } \\
\text { w/o Rn }\end{array}$ & Soil Ingestion \\
\hline & & & & \\
$5 \%$ & $3.96 \mathrm{E}-02$ & $3.96 \mathrm{E}-02$ & $1.87 \mathrm{E}-08$ & $2.00 \mathrm{E}-06$ \\
$10 \%$ & $1.16 \mathrm{E}-01$ & $1.16 \mathrm{E}-01$ & $4.45 \mathrm{E}-08$ & $4.74 \mathrm{E}-06$ \\
$15 \%$ & $1.93 \mathrm{E}-01$ & $1.93 \mathrm{E}-01$ & $6.96 \mathrm{E}-08$ & $7.27 \mathrm{E}-06$ \\
$20 \%$ & $2.69 \mathrm{E}-01$ & $2.69 \mathrm{E}-01$ & $9.28 \mathrm{E}-08$ & $9.57 \mathrm{E}-06$ \\
$25 \%$ & $3.40 \mathrm{E}-01$ & $3.40 \mathrm{E}-01$ & $1.14 \mathrm{E}-07$ & $1.20 \mathrm{E}-05$ \\
$30 \%$ & $3.61 \mathrm{E}-01$ & $3.61 \mathrm{E}-01$ & $1.31 \mathrm{E}-07$ & $1.41 \mathrm{E}-05$ \\
$35 \%$ & $3.74 \mathrm{E}-01$ & $3.74 \mathrm{E}-01$ & $1.45 \mathrm{E}-07$ & $1.63 \mathrm{E}-05$ \\
$40 \%$ & $3.88 \mathrm{E}-01$ & $3.88 \mathrm{E}-01$ & $1.58 \mathrm{E}-07$ & $1.82 \mathrm{E}-05$ \\
$45 \%$ & $4.01 \mathrm{E}-01$ & $4.01 \mathrm{E}-01$ & $1.71 \mathrm{E}-07$ & $2.01 \mathrm{E}-05$ \\
$50 \%$ & $4.14 \mathrm{E}-01$ & $4.14 \mathrm{E}-01$ & $1.83 \mathrm{E}-07$ & $2.18 \mathrm{E}-05$ \\
$55 \%$ & $4.24 \mathrm{E}-01$ & $4.24 \mathrm{E}-01$ & $1.98 \mathrm{E}-07$ & $2.35 \mathrm{E}-05$ \\
$60 \%$ & $4.33 \mathrm{E}-01$ & $4.33 \mathrm{E}-01$ & $2.15 \mathrm{E}-07$ & $2.53 \mathrm{E}-05$ \\
$65 \%$ & $4.41 \mathrm{E}-01$ & $4.41 \mathrm{E}-01$ & $2.34 \mathrm{E}-07$ & $2.69 \mathrm{E}-05$ \\
$70 \%$ & $4.50 \mathrm{E}-01$ & $4.50 \mathrm{E}-01$ & $2.54 \mathrm{E}-07$ & $2.89 \mathrm{E}-05$ \\
$75 \%$ & $4.60 \mathrm{E}-01$ & $4.60 \mathrm{E}-01$ & $2.80 \mathrm{E}-07$ & $3.11 \mathrm{E}-05$ \\
$80 \%$ & $4.84 \mathrm{E}-01$ & $4.84 \mathrm{E}-01$ & $3.09 \mathrm{E}-07$ & $3.35 \mathrm{E}-05$ \\
$85 \%$ & $5.09 \mathrm{E}-01$ & $5.09 \mathrm{E}-01$ & $3.47 \mathrm{E}-07$ & $3.63 \mathrm{E}-05$ \\
$90 \%$ & $5.34 \mathrm{E}-01$ & $5.34 \mathrm{E}-01$ & $4.02 \mathrm{E}-07$ & $4.00 \mathrm{E}-05$ \\
$95 \%$ & $5.70 \mathrm{E}-01$ & $5.70 \mathrm{E}-01$ & $4.90 \mathrm{E}-07$ & $4.55 \mathrm{E}-05$ \\
Mean & $3.80 \mathrm{E}-01$ & $3.80 \mathrm{E}-01$ & $2.12 \mathrm{E}-07$ & $2.23 \mathrm{E}-05$ \\
Min & $3.50 \mathrm{E}-03$ & $3.50 \mathrm{E}-03$ & $1.15 \mathrm{E}-10$ & $4.07 \mathrm{E}-08$ \\
Max & $7.91 \mathrm{E}-01$ & $7.91 \mathrm{E}-01$ & $1.66 \mathrm{E}-06$ & $8.39 \mathrm{E}-05$ \\
\hline & & & & \\
\hline
\end{tabular}


TABLE C.3 Peak DSR Percentiles (mrem/yr per pCi/g) for Different Exposure Pathways for C-14 in Industrial Use Scenario

\begin{tabular}{rrrrr}
\hline & & & & \\
Percentile & All Pathways & External & $\begin{array}{c}\text { Inhalation } \\
\text { w/o Rn }\end{array}$ & Soil Ingestion \\
\hline & & & & \\
$5 \%$ & $2.22 \mathrm{E}-06$ & $3.91 \mathrm{E}-08$ & $1.94 \mathrm{E}-06$ & $1.19 \mathrm{E}-07$ \\
$10 \%$ & $5.82 \mathrm{E}-06$ & $1.14 \mathrm{E}-07$ & $5.28 \mathrm{E}-06$ & $2.85 \mathrm{E}-07$ \\
$15 \%$ & $1.02 \mathrm{E}-05$ & $1.89 \mathrm{E}-07$ & $9.27 \mathrm{E}-06$ & $4.35 \mathrm{E}-07$ \\
$20 \%$ & $1.34 \mathrm{E}-05$ & $2.64 \mathrm{E}-07$ & $1.20 \mathrm{E}-05$ & $5.79 \mathrm{E}-07$ \\
$25 \%$ & $1.53 \mathrm{E}-05$ & $3.37 \mathrm{E}-07$ & $1.38 \mathrm{E}-05$ & $7.13 \mathrm{E}-07$ \\
$30 \%$ & $1.68 \mathrm{E}-05$ & $3.51 \mathrm{E}-07$ & $1.50 \mathrm{E}-05$ & $8.45 \mathrm{E}-07$ \\
$35 \%$ & $1.79 \mathrm{E}-05$ & $3.65 \mathrm{E}-07$ & $1.62 \mathrm{E}-05$ & $9.74 \mathrm{E}-07$ \\
$40 \%$ & $1.90 \mathrm{E}-05$ & $3.78 \mathrm{E}-07$ & $1.72 \mathrm{E}-05$ & $1.10 \mathrm{E}-06$ \\
$45 \%$ & $2.02 \mathrm{E}-05$ & $3.90 \mathrm{E}-07$ & $1.84 \mathrm{E}-05$ & $1.20 \mathrm{E}-06$ \\
$50 \%$ & $2.13 \mathrm{E}-05$ & $4.02 \mathrm{E}-07$ & $1.93 \mathrm{E}-05$ & $1.31 \mathrm{E}-06$ \\
$55 \%$ & $2.23 \mathrm{E}-05$ & $4.12 \mathrm{E}-07$ & $2.03 \mathrm{E}-05$ & $1.41 \mathrm{E}-06$ \\
$60 \%$ & $2.34 \mathrm{E}-05$ & $4.21 \mathrm{E}-07$ & $2.14 \mathrm{E}-05$ & $1.52 \mathrm{E}-06$ \\
$65 \%$ & $2.45 \mathrm{E}-05$ & $4.28 \mathrm{E}-07$ & $2.24 \mathrm{E}-05$ & $1.61 \mathrm{E}-06$ \\
$70 \%$ & $2.56 \mathrm{E}-05$ & $4.37 \mathrm{E}-07$ & $2.36 \mathrm{E}-05$ & $1.73 \mathrm{E}-06$ \\
$75 \%$ & $2.70 \mathrm{E}-05$ & $4.47 \mathrm{E}-07$ & $2.50 \mathrm{E}-05$ & $1.86 \mathrm{E}-06$ \\
$80 \%$ & $2.87 \mathrm{E}-05$ & $4.69 \mathrm{E}-07$ & $2.64 \mathrm{E}-05$ & $2.00 \mathrm{E}-06$ \\
$85 \%$ & $3.05 \mathrm{E}-05$ & $4.93 \mathrm{E}-07$ & $2.83 \mathrm{E}-05$ & $2.18 \mathrm{E}-06$ \\
$90 \%$ & $3.32 \mathrm{E}-05$ & $5.18 \mathrm{E}-07$ & $3.11 \mathrm{E}-05$ & $2.39 \mathrm{E}-06$ \\
$95 \%$ & $3.73 \mathrm{E}-05$ & $5.54 \mathrm{E}-07$ & $3.50 \mathrm{E}-05$ & $2.71 \mathrm{E}-06$ \\
Mean & $2.10 \mathrm{E}-05$ & $3.69 \mathrm{E}-07$ & $1.93 \mathrm{E}-05$ & $1.34 \mathrm{E}-06$ \\
Min & $1.45 \mathrm{E}-07$ & $3.37 \mathrm{E}-09$ & $1.35 \mathrm{E}-07$ & $2.41 \mathrm{E}-09$ \\
Max & $7.84 \mathrm{E}-05$ & $7.68 \mathrm{E}-07$ & $7.40 \mathrm{E}-05$ & $5.03 \mathrm{E}-06$ \\
\hline & & & & \\
\hline
\end{tabular}


TABLE C.4 Peak DSR Percentiles (mrem/yr per pCi/g) for Different Exposure Pathways for Cs-137 in Industrial Use Scenario

\begin{tabular}{ccccc}
\hline & & & & \\
Percentile & All Pathways & External & $\begin{array}{c}\text { Inhalation } \\
\text { w/o Rn }\end{array}$ & Soil Ingestion \\
\hline $5 \%$ & $7.12 \mathrm{E}-02$ & $7.11 \mathrm{E}-02$ & $7.46 \mathrm{E}-08$ & $1.77 \mathrm{E}-05$ \\
$10 \%$ & $2.09 \mathrm{E}-01$ & $2.08 \mathrm{E}-01$ & $1.79 \mathrm{E}-07$ & $4.24 \mathrm{E}-05$ \\
$15 \%$ & $3.47 \mathrm{E}-01$ & $3.47 \mathrm{E}-01$ & $2.79 \mathrm{E}-07$ & $6.46 \mathrm{E}-05$ \\
$20 \%$ & $4.85 \mathrm{E}-01$ & $4.85 \mathrm{E}-01$ & $3.76 \mathrm{E}-07$ & $8.58 \mathrm{E}-05$ \\
$25 \%$ & $6.22 \mathrm{E}-01$ & $6.22 \mathrm{E}-01$ & $4.58 \mathrm{E}-07$ & $1.06 \mathrm{E}-04$ \\
$30 \%$ & $6.46 \mathrm{E}-01$ & $6.46 \mathrm{E}-01$ & $5.23 \mathrm{E}-07$ & $1.26 \mathrm{E}-04$ \\
$35 \%$ & $6.70 \mathrm{E}-01$ & $6.69 \mathrm{E}-01$ & $5.77 \mathrm{E}-07$ & $1.46 \mathrm{E}-04$ \\
$40 \%$ & $6.93 \mathrm{E}-01$ & $6.93 \mathrm{E}-01$ & $6.30 \mathrm{E}-07$ & $1.63 \mathrm{E}-04$ \\
$45 \%$ & $7.17 \mathrm{E}-01$ & $7.17 \mathrm{E}-01$ & $6.84 \mathrm{E}-07$ & $1.79 \mathrm{E}-04$ \\
$50 \%$ & $7.41 \mathrm{E}-01$ & $7.40 \mathrm{E}-01$ & $7.32 \mathrm{E}-07$ & $1.94 \mathrm{E}-04$ \\
$55 \%$ & $7.56 \mathrm{E}-01$ & $7.56 \mathrm{E}-01$ & $7.90 \mathrm{E}-07$ & $2.09 \mathrm{E}-04$ \\
$60 \%$ & $7.71 \mathrm{E}-01$ & $7.71 \mathrm{E}-01$ & $8.60 \mathrm{E}-07$ & $2.25 \mathrm{E}-04$ \\
$65 \%$ & $7.87 \mathrm{E}-01$ & $7.87 \mathrm{E}-01$ & $9.37 \mathrm{E}-07$ & $2.40 \mathrm{E}-04$ \\
$70 \%$ & $8.02 \mathrm{E}-01$ & $8.02 \mathrm{E}-01$ & $1.01 \mathrm{E}-06$ & $2.58 \mathrm{E}-04$ \\
$75 \%$ & $8.18 \mathrm{E}-01$ & $8.17 \mathrm{E}-01$ & $1.12 \mathrm{E}-06$ & $2.78 \mathrm{E}-04$ \\
$80 \%$ & $8.62 \mathrm{E}-01$ & $8.62 \mathrm{E}-01$ & $1.23 \mathrm{E}-06$ & $2.98 \mathrm{E}-04$ \\
$85 \%$ & $9.06 \mathrm{E}-01$ & $9.06 \mathrm{E}-01$ & $1.39 \mathrm{E}-06$ & $3.24 \mathrm{E}-04$ \\
$90 \%$ & $9.51 \mathrm{E}-01$ & $9.51 \mathrm{E}-01$ & $1.60 \mathrm{E}-06$ & $3.56 \mathrm{E}-04$ \\
$95 \%$ & $1.01 \mathrm{E}+00$ & $1.01 \mathrm{E}+00$ & $1.96 \mathrm{E}-06$ & $4.03 \mathrm{E}-04$ \\
Mean & $6.78 \mathrm{E}-01$ & $6.78 \mathrm{E}-01$ & $8.46 \mathrm{E}-07$ & $1.99 \mathrm{E}-04$ \\
Min & $6.21 \mathrm{E}-03$ & $6.20 \mathrm{E}-03$ & $4.58 \mathrm{E}-10$ & $3.60 \mathrm{E}-07$ \\
Max & $1.40 \mathrm{E}+00$ & $1.40 \mathrm{E}+00$ & $6.61 \mathrm{E}-06$ & $7.42 \mathrm{E}-04$ \\
\hline & & & & \\
\hline
\end{tabular}


TABLE C.5 Peak DSR Percentiles (mrem/yr per pCi/g) for Different Exposure Pathways for Pu-238 in Industrial Use Scenario

\begin{tabular}{rccccc}
\hline & & & & \\
Percentile & All Pathways & External & $\begin{array}{c}\text { Inhation } \\
\text { w/o Rn }\end{array}$ & Radon (WI) & Soil Ingestion \\
\hline $5 \%$ & $6.12 \mathrm{E}-04$ & $2.76 \mathrm{E}-06$ & $2.13 \mathrm{E}-04$ & $1.01 \mathrm{E}-11$ & $3.16 \mathrm{E}-04$ \\
$10 \%$ & $1.65 \mathrm{E}-03$ & $8.08 \mathrm{E}-06$ & $5.09 \mathrm{E}-04$ & $2.50 \mathrm{E}-11$ & $7.56 \mathrm{E}-04$ \\
$15 \%$ & $2.51 \mathrm{E}-03$ & $1.35 \mathrm{E}-05$ & $7.94 \mathrm{E}-04$ & $4.50 \mathrm{E}-11$ & $1.15 \mathrm{E}-03$ \\
$20 \%$ & $3.24 \mathrm{E}-03$ & $1.88 \mathrm{E}-05$ & $1.07 \mathrm{E}-03$ & $7.21 \mathrm{E}-11$ & $1.53 \mathrm{E}-03$ \\
$25 \%$ & $3.85 \mathrm{E}-03$ & $2.41 \mathrm{E}-05$ & $1.30 \mathrm{E}-03$ & $9.43 \mathrm{E}-11$ & $1.89 \mathrm{E}-03$ \\
$30 \%$ & $4.36 \mathrm{E}-03$ & $2.50 \mathrm{E}-05$ & $1.49 \mathrm{E}-03$ & $1.19 \mathrm{E}-10$ & $2.24 \mathrm{E}-03$ \\
$35 \%$ & $4.81 \mathrm{E}-03$ & $2.60 \mathrm{E}-05$ & $1.64 \mathrm{E}-03$ & $1.41 \mathrm{E}-10$ & $2.60 \mathrm{E}-03$ \\
$40 \%$ & $5.21 \mathrm{E}-03$ & $2.69 \mathrm{E}-05$ & $1.79 \mathrm{E}-03$ & $1.62 \mathrm{E}-10$ & $2.91 \mathrm{E}-03$ \\
$45 \%$ & $5.56 \mathrm{E}-03$ & $2.78 \mathrm{E}-05$ & $1.95 \mathrm{E}-03$ & $1.81 \mathrm{E}-10$ & $3.19 \mathrm{E}-03$ \\
$50 \%$ & $5.92 \mathrm{E}-03$ & $2.87 \mathrm{E}-05$ & $2.08 \mathrm{E}-03$ & $2.03 \mathrm{E}-10$ & $3.46 \mathrm{E}-03$ \\
$55 \%$ & $6.27 \mathrm{E}-03$ & $2.93 \mathrm{E}-05$ & $2.25 \mathrm{E}-03$ & $2.24 \mathrm{E}-10$ & $3.73 \mathrm{E}-03$ \\
$60 \%$ & $6.65 \mathrm{E}-03$ & $2.99 \mathrm{E}-05$ & $2.45 \mathrm{E}-03$ & $2.48 \mathrm{E}-10$ & $4.02 \mathrm{E}-03$ \\
$65 \%$ & $7.07 \mathrm{E}-03$ & $3.05 \mathrm{E}-05$ & $2.67 \mathrm{E}-03$ & $2.74 \mathrm{E}-10$ & $4.27 \mathrm{E}-03$ \\
$70 \%$ & $7.54 \mathrm{E}-03$ & $3.11 \mathrm{E}-05$ & $2.89 \mathrm{E}-03$ & $3.05 \mathrm{E}-10$ & $4.58 \mathrm{E}-03$ \\
$75 \%$ & $7.97 \mathrm{E}-03$ & $3.17 \mathrm{E}-05$ & $3.18 \mathrm{E}-03$ & $3.37 \mathrm{E}-10$ & $4.95 \mathrm{E}-03$ \\
$80 \%$ & $8.46 \mathrm{E}-03$ & $3.34 \mathrm{E}-05$ & $3.52 \mathrm{E}-03$ & $3.76 \mathrm{E}-10$ & $5.32 \mathrm{E}-03$ \\
$85 \%$ & $9.12 \mathrm{E}-03$ & $3.52 \mathrm{E}-05$ & $3.95 \mathrm{E}-03$ & $4.23 \mathrm{E}-10$ & $5.77 \mathrm{E}-03$ \\
$90 \%$ & $1.00 \mathrm{E}-02$ & $3.69 \mathrm{E}-05$ & $4.57 \mathrm{E}-03$ & $4.90 \mathrm{E}-10$ & $6.33 \mathrm{E}-03$ \\
$95 \%$ & $1.13 \mathrm{E}-02$ & $3.93 \mathrm{E}-05$ & $5.57 \mathrm{E}-03$ & $6.08 \mathrm{E}-10$ & $7.19 \mathrm{E}-03$ \\
Mean & $5.98 \mathrm{E}-03$ & $2.63 \mathrm{E}-05$ & $2.41 \mathrm{E}-03$ & $2.41 \mathrm{E}-10$ & $3.54 \mathrm{E}-03$ \\
Min & $2.74 \mathrm{E}-05$ & $2.41 \mathrm{E}-07$ & $1.30 \mathrm{E}-06$ & $9.48 \mathrm{E}-14$ & $6.42 \mathrm{E}-06$ \\
Max & $2.76 \mathrm{E}-02$ & $5.44 \mathrm{E}-05$ & $1.88 \mathrm{E}-02$ & $2.03 \mathrm{E}-09$ & $1.32 \mathrm{E}-02$ \\
\hline & & & & & \\
\hline
\end{tabular}


TABLE C.6 Peak DSR Percentiles (mrem/yr per pCi/g) for Different Exposure Pathways for Pu-239 in Industrial Use Scenario

\begin{tabular}{rcccc}
\hline & & & & \\
Percentile & All Pathways & External & $\begin{array}{c}\text { Inhalation } \\
\text { w/o Rn }\end{array}$ & Soil Ingestion \\
\hline & & & & \\
$5 \%$ & $6.73 \mathrm{E}-04$ & $6.04 \mathrm{E}-06$ & $2.33 \mathrm{E}-04$ & $3.45 \mathrm{E}-04$ \\
$10 \%$ & $1.81 \mathrm{E}-03$ & $1.77 \mathrm{E}-05$ & $5.58 \mathrm{E}-04$ & $8.25 \mathrm{E}-04$ \\
$15 \%$ & $2.76 \mathrm{E}-03$ & $2.94 \mathrm{E}-05$ & $8.69 \mathrm{E}-04$ & $1.26 \mathrm{E}-03$ \\
$20 \%$ & $3.57 \mathrm{E}-03$ & $4.12 \mathrm{E}-05$ & $1.17 \mathrm{E}-03$ & $1.67 \mathrm{E}-03$ \\
$25 \%$ & $4.24 \mathrm{E}-03$ & $5.28 \mathrm{E}-05$ & $1.43 \mathrm{E}-03$ & $2.06 \mathrm{E}-03$ \\
$30 \%$ & $4.79 \mathrm{E}-03$ & $5.48 \mathrm{E}-05$ & $1.63 \mathrm{E}-03$ & $2.44 \mathrm{E}-03$ \\
$35 \%$ & $5.27 \mathrm{E}-03$ & $5.68 \mathrm{E}-05$ & $1.80 \mathrm{E}-03$ & $2.84 \mathrm{E}-03$ \\
$40 \%$ & $5.72 \mathrm{E}-03$ & $5.88 \mathrm{E}-05$ & $1.97 \mathrm{E}-03$ & $3.17 \mathrm{E}-03$ \\
$45 \%$ & $6.11 \mathrm{E}-03$ & $6.08 \mathrm{E}-05$ & $2.13 \mathrm{E}-03$ & $3.48 \mathrm{E}-03$ \\
$50 \%$ & $6.51 \mathrm{E}-03$ & $6.28 \mathrm{E}-05$ & $2.28 \mathrm{E}-03$ & $3.78 \mathrm{E}-03$ \\
$55 \%$ & $6.89 \mathrm{E}-03$ & $6.41 \mathrm{E}-05$ & $2.46 \mathrm{E}-03$ & $4.07 \mathrm{E}-03$ \\
$60 \%$ & $7.30 \mathrm{E}-03$ & $6.54 \mathrm{E}-05$ & $2.68 \mathrm{E}-03$ & $4.39 \mathrm{E}-03$ \\
$65 \%$ & $7.76 \mathrm{E}-03$ & $6.67 \mathrm{E}-05$ & $2.92 \mathrm{E}-03$ & $4.66 \mathrm{E}-03$ \\
$70 \%$ & $8.27 \mathrm{E}-03$ & $6.81 \mathrm{E}-05$ & $3.16 \mathrm{E}-03$ & $5.01 \mathrm{E}-03$ \\
$75 \%$ & $8.75 \mathrm{E}-03$ & $6.94 \mathrm{E}-05$ & $3.49 \mathrm{E}-03$ & $5.40 \mathrm{E}-03$ \\
$80 \%$ & $9.28 \mathrm{E}-03$ & $7.31 \mathrm{E}-05$ & $3.85 \mathrm{E}-03$ & $5.81 \mathrm{E}-03$ \\
$85 \%$ & $1.00 \mathrm{E}-02$ & $7.69 \mathrm{E}-05$ & $4.32 \mathrm{E}-03$ & $6.30 \mathrm{E}-03$ \\
$90 \%$ & $1.10 \mathrm{E}-02$ & $8.07 \mathrm{E}-05$ & $5.00 \mathrm{E}-03$ & $6.91 \mathrm{E}-03$ \\
$95 \%$ & $1.24 \mathrm{E}-02$ & $8.59 \mathrm{E}-05$ & $6.10 \mathrm{E}-03$ & $7.85 \mathrm{E}-03$ \\
Mean & $6.56 \mathrm{E}-03$ & $5.75 \mathrm{E}-05$ & $2.64 \mathrm{E}-03$ & $3.87 \mathrm{E}-03$ \\
Min & $3.02 \mathrm{E}-05$ & $5.27 \mathrm{E}-07$ & $1.43 \mathrm{E}-06$ & $7.00 \mathrm{E}-06$ \\
Max & $3.02 \mathrm{E}-02$ & $1.19 \mathrm{E}-04$ & $2.06 \mathrm{E}-02$ & $1.44 \mathrm{E}-02$ \\
\hline & & & & \\
\hline
\end{tabular}


TABLE C.7 Peak DSR Percentiles (mrem/yr per pCi/g) for Different Exposure Pathways for Sr-90 in Industrial Use Scenario

\begin{tabular}{ccccc}
\hline & & & & \\
Percentile & All Pathways & External & $\begin{array}{c}\text { Inhalation } \\
\text { w/o Rn }\end{array}$ & Soil Ingestion \\
\hline & & & & \\
$5 \%$ & $9.62 \mathrm{E}-04$ & $9.03 \mathrm{E}-04$ & $3.08 \mathrm{E}-07$ & $4.18 \mathrm{E}-05$ \\
$10 \%$ & $2.84 \mathrm{E}-03$ & $2.67 \mathrm{E}-03$ & $7.35 \mathrm{E}-07$ & $1.00 \mathrm{E}-04$ \\
$15 \%$ & $4.65 \mathrm{E}-03$ & $4.42 \mathrm{E}-03$ & $1.15 \mathrm{E}-06$ & $1.53 \mathrm{E}-04$ \\
$20 \%$ & $6.54 \mathrm{E}-03$ & $6.18 \mathrm{E}-03$ & $1.55 \mathrm{E}-06$ & $2.02 \mathrm{E}-04$ \\
$25 \%$ & $8.25 \mathrm{E}-03$ & $7.91 \mathrm{E}-03$ & $1.89 \mathrm{E}-06$ & $2.51 \mathrm{E}-04$ \\
$30 \%$ & $8.72 \mathrm{E}-03$ & $8.27 \mathrm{E}-03$ & $2.16 \mathrm{E}-06$ & $2.96 \mathrm{E}-04$ \\
$35 \%$ & $9.02 \mathrm{E}-03$ & $8.58 \mathrm{E}-03$ & $2.38 \mathrm{E}-06$ & $3.44 \mathrm{E}-04$ \\
$40 \%$ & $9.35 \mathrm{E}-03$ & $8.86 \mathrm{E}-03$ & $2.60 \mathrm{E}-06$ & $3.84 \mathrm{E}-04$ \\
$45 \%$ & $9.66 \mathrm{E}-03$ & $9.17 \mathrm{E}-03$ & $2.82 \mathrm{E}-06$ & $4.22 \mathrm{E}-04$ \\
$50 \%$ & $9.94 \mathrm{E}-03$ & $9.47 \mathrm{E}-03$ & $3.03 \mathrm{E}-06$ & $4.57 \mathrm{E}-04$ \\
$55 \%$ & $1.02 \mathrm{E}-02$ & $9.68 \mathrm{E}-03$ & $3.26 \mathrm{E}-06$ & $4.94 \mathrm{E}-04$ \\
$60 \%$ & $1.04 \mathrm{E}-02$ & $9.87 \mathrm{E}-03$ & $3.55 \mathrm{E}-06$ & $5.32 \mathrm{E}-04$ \\
$65 \%$ & $1.06 \mathrm{E}-02$ & $1.01 \mathrm{E}-02$ & $3.88 \mathrm{E}-06$ & $5.66 \mathrm{E}-04$ \\
$70 \%$ & $1.09 \mathrm{E}-02$ & $1.03 \mathrm{E}-02$ & $4.20 \mathrm{E}-06$ & $6.07 \mathrm{E}-04$ \\
$75 \%$ & $1.12 \mathrm{E}-02$ & $1.05 \mathrm{E}-02$ & $4.63 \mathrm{E}-06$ & $6.54 \mathrm{E}-04$ \\
$80 \%$ & $1.16 \mathrm{E}-02$ & $1.10 \mathrm{E}-02$ & $5.11 \mathrm{E}-06$ & $7.04 \mathrm{E}-04$ \\
$85 \%$ & $1.22 \mathrm{E}-02$ & $1.16 \mathrm{E}-02$ & $5.71 \mathrm{E}-06$ & $7.62 \mathrm{E}-04$ \\
$90 \%$ & $1.29 \mathrm{E}-02$ & $1.22 \mathrm{E}-02$ & $6.63 \mathrm{E}-06$ & $8.34 \mathrm{E}-04$ \\
$95 \%$ & $1.38 \mathrm{E}-02$ & $1.30 \mathrm{E}-02$ & $8.09 \mathrm{E}-06$ & $9.52 \mathrm{E}-04$ \\
Mean & $9.15 \mathrm{E}-03$ & $8.68 \mathrm{E}-03$ & $3.49 \mathrm{E}-06$ & $4.69 \mathrm{E}-04$ \\
Min & $7.80 \mathrm{E}-05$ & $7.57 \mathrm{E}-05$ & $1.90 \mathrm{E}-09$ & $8.52 \mathrm{E}-07$ \\
Max & $1.96 \mathrm{E}-02$ & $1.80 \mathrm{E}-02$ & $2.74 \mathrm{E}-05$ & $1.76 \mathrm{E}-03$ \\
\hline & & & & \\
\hline
\end{tabular}




\section{TABLE C.8 Peak DSR Percentiles (mrem/yr per pCi/g) for Different Exposure Pathways for Tc-99 in Industrial Use Scenario}

\begin{tabular}{rrrrr}
\hline & & & & \\
Percentile & All Pathways & External & w/o Rn & Soil Ingestion \\
\hline & & & & \\
$5 \%$ & $3.44 \mathrm{E}-06$ & $2.35 \mathrm{E}-06$ & $2.37 \mathrm{E}-08$ & $8.40 \mathrm{E}-07$ \\
$10 \%$ & $9.83 \mathrm{E}-06$ & $6.91 \mathrm{E}-06$ & $5.70 \mathrm{E}-08$ & $2.00 \mathrm{E}-06$ \\
$15 \%$ & $1.62 \mathrm{E}-05$ & $1.15 \mathrm{E}-05$ & $8.88 \mathrm{E}-08$ & $3.05 \mathrm{E}-06$ \\
$20 \%$ & $2.27 \mathrm{E}-05$ & $1.61 \mathrm{E}-05$ & $1.19 \mathrm{E}-07$ & $4.06 \mathrm{E}-06$ \\
$25 \%$ & $2.65 \mathrm{E}-05$ & $2.05 \mathrm{E}-05$ & $1.46 \mathrm{E}-07$ & $5.00 \mathrm{E}-06$ \\
$30 \%$ & $2.87 \mathrm{E}-05$ & $2.14 \mathrm{E}-05$ & $1.67 \mathrm{E}-07$ & $5.90 \mathrm{E}-06$ \\
$35 \%$ & $3.04 \mathrm{E}-05$ & $2.22 \mathrm{E}-05$ & $1.84 \mathrm{E}-07$ & $6.85 \mathrm{E}-06$ \\
$40 \%$ & $3.18 \mathrm{E}-05$ & $2.30 \mathrm{E}-05$ & $2.02 \mathrm{E}-07$ & $7.71 \mathrm{E}-06$ \\
$45 \%$ & $3.30 \mathrm{E}-05$ & $2.38 \mathrm{E}-05$ & $2.17 \mathrm{E}-07$ & $8.44 \mathrm{E}-06$ \\
$50 \%$ & $3.41 \mathrm{E}-05$ & $2.45 \mathrm{E}-05$ & $2.34 \mathrm{E}-07$ & $9.17 \mathrm{E}-06$ \\
$55 \%$ & $3.54 \mathrm{E}-05$ & $2.51 \mathrm{E}-05$ & $2.52 \mathrm{E}-07$ & $9.88 \mathrm{E}-06$ \\
$60 \%$ & $3.65 \mathrm{E}-05$ & $2.56 \mathrm{E}-05$ & $2.75 \mathrm{E}-07$ & $1.06 \mathrm{E}-05$ \\
$65 \%$ & $3.77 \mathrm{E}-05$ & $2.61 \mathrm{E}-05$ & $2.99 \mathrm{E}-07$ & $1.13 \mathrm{E}-05$ \\
$70 \%$ & $3.89 \mathrm{E}-05$ & $2.66 \mathrm{E}-05$ & $3.25 \mathrm{E}-07$ & $1.21 \mathrm{E}-05$ \\
$75 \%$ & $4.02 \mathrm{E}-05$ & $2.73 \mathrm{E}-05$ & $3.58 \mathrm{E}-07$ & $1.31 \mathrm{E}-05$ \\
$80 \%$ & $4.18 \mathrm{E}-05$ & $2.85 \mathrm{E}-05$ & $3.94 \mathrm{E}-07$ & $1.41 \mathrm{E}-05$ \\
$85 \%$ & $4.38 \mathrm{E}-05$ & $3.00 \mathrm{E}-05$ & $4.42 \mathrm{E}-07$ & $1.53 \mathrm{E}-05$ \\
$90 \%$ & $4.70 \mathrm{E}-05$ & $3.16 \mathrm{E}-05$ & $5.10 \mathrm{E}-07$ & $1.67 \mathrm{E}-05$ \\
$95 \%$ & $5.18 \mathrm{E}-05$ & $3.39 \mathrm{E}-05$ & $6.23 \mathrm{E}-07$ & $1.90 \mathrm{E}-05$ \\
Mean & $3.21 \mathrm{E}-05$ & $2.25 \mathrm{E}-05$ & $2.70 \mathrm{E}-07$ & $9.37 \mathrm{E}-06$ \\
Min & $2.52 \mathrm{E}-07$ & $2.01 \mathrm{E}-07$ & $1.50 \mathrm{E}-10$ & $1.72 \mathrm{E}-08$ \\
Max & $8.21 \mathrm{E}-05$ & $4.69 \mathrm{E}-05$ & $2.13 \mathrm{E}-06$ & $3.57 \mathrm{E}-05$ \\
\hline & & & & \\
\hline
\end{tabular}

Tables C.9-C.16 show the peak DSR percentiles (mrem/yr per pCi/g) for different exposure pathways for individual radionuclides in the offsite resident scenario. RESRADOFFSITE version 2.6 was used in the analysis. For all radionuclides except Ba-133, waterdependent pathways were dominant, and for Ba-133, the water-independent pathway was dominant. For Am-241, Pu-238, and Pu-239, fish and plant ingestion were dominant exposure pathways. For Ba-133, the external exposure pathway was dominant. For C-14, aquatic food ingestion resulted in most dose. For Cs-137, fish, meat, and milk ingestion resulted in most dose. For Sr-90, fish, meat, and plant ingestion resulted in most dose. For Tc-99, plant, fish, and milk ingestion resulted in most dose. 


\section{TABLE C.9 Peak DSR Percentiles (mrem/yr per pCi/g) for Different Exposure Pathways for Am-241 in Offsite Resident Scenario}

\begin{tabular}{|c|c|c|c|c|c|c|c|c|c|c|c|c|c|}
\hline Percentile & Total & External-WD & Fish & Plant-WD & Meat-WD & Milk-WD & Soil-WD & External-WI & Inhalation & Plant-WI & Meat-WI & Milk-WI & Soil-WI \\
\hline $5 \%$ & $2.16 \mathrm{E}-03$ & $2.72 \mathrm{E}-06$ & $1.99 \mathrm{E}-03$ & $1.21 \mathrm{E}-04$ & $2.90 \mathrm{E}-06$ & $3.07 \mathrm{E}-07$ & $9.24 \mathrm{E}-07$ & 9.69E-08 & $6.63 \mathrm{E}-06$ & 5.17E-09 & $3.51 \mathrm{E}-11$ & $1.72 \mathrm{E}-12$ & $2.15 \mathrm{E}-10$ \\
\hline $10 \%$ & $2.69 \mathrm{E}-03$ & $5.72 \mathrm{E}-06$ & $2.47 \mathrm{E}-03$ & $1.62 \mathrm{E}-04$ & $3.68 \mathrm{E}-06$ & $4.41 \mathrm{E}-07$ & $1.95 \mathrm{E}-06$ & $9.70 \mathrm{E}-08$ & $6.64 \mathrm{E}-06$ & $6.08 \mathrm{E}-09$ & $3.86 \mathrm{E}-11$ & $2.24 \mathrm{E}-12$ & $3.08 \mathrm{E}-10$ \\
\hline $15 \%$ & $3.32 \mathrm{E}-03$ & $8.77 \mathrm{E}-06$ & $3.07 \mathrm{E}-03$ & $2.00 \mathrm{E}-04$ & $4.50 \mathrm{E}-06$ & $5.44 \mathrm{E}-07$ & $2.96 \mathrm{E}-06$ & $9.70 \mathrm{E}-08$ & $6.64 \mathrm{E}-06$ & $6.76 \mathrm{E}-09$ & $4.15 \mathrm{E}-11$ & $2.66 \mathrm{E}-12$ & $3.89 \mathrm{E}-10$ \\
\hline $20 \%$ & 4.17E-03 & $1.18 \mathrm{E}-05$ & $3.85 \mathrm{E}-03$ & $2.35 \mathrm{E}-04$ & $5.50 \mathrm{E}-06$ & $6.53 \mathrm{E}-07$ & $4.01 \mathrm{E}-06$ & $9.70 \mathrm{E}-08$ & $6.64 \mathrm{E}-06$ & $7.40 \mathrm{E}-09$ & $4.41 \mathrm{E}-11$ & $3.02 \mathrm{E}-12$ & $4.80 \mathrm{E}-10$ \\
\hline $25 \%$ & $4.91 \mathrm{E}-03$ & $1.46 \mathrm{E}-05$ & $4.56 \mathrm{E}-03$ & $2.68 \mathrm{E}-04$ & $6.61 \mathrm{E}-06$ & $7.47 \mathrm{E}-07$ & $4.96 \mathrm{E}-06$ & $9.70 \mathrm{E}-08$ & $6.64 \mathrm{E}-06$ & $8.02 \mathrm{E}-09$ & 4.63E-11 & $3.43 \mathrm{E}-12$ & $5.47 \mathrm{E}-10$ \\
\hline $30 \%$ & $5.62 \mathrm{E}-03$ & $1.69 \mathrm{E}-05$ & $5.21 \mathrm{E}-03$ & $2.97 \mathrm{E}-04$ & 7.37E-06 & $8.52 \mathrm{E}-07$ & $5.92 \mathrm{E}-06$ & $9.70 \mathrm{E}-08$ & $6.64 \mathrm{E}-06$ & $8.68 \mathrm{E}-09$ & $4.88 \mathrm{E}-11$ & $3.80 \mathrm{E}-12$ & $6.16 \mathrm{E}-10$ \\
\hline $35 \%$ & $6.21 \mathrm{E}-03$ & $1.94 \mathrm{E}-05$ & $5.75 \mathrm{E}-03$ & $3.30 \mathrm{E}-04$ & 8.04E-06 & $9.51 \mathrm{E}-07$ & $6.97 \mathrm{E}-06$ & $9.70 \mathrm{E}-08$ & $6.64 \mathrm{E}-06$ & $9.27 \mathrm{E}-09$ & $5.07 \mathrm{E}-11$ & $4.21 \mathrm{E}-12$ & $6.85 \mathrm{E}-10$ \\
\hline $40 \%$ & $6.66 \mathrm{E}-03$ & 2.19E-05 & $6.14 \mathrm{E}-03$ & $3.58 \mathrm{E}-04$ & $8.62 \mathrm{E}-06$ & $1.05 \mathrm{E}-06$ & $8.13 \mathrm{E}-06$ & $9.70 \mathrm{E}-08$ & $6.64 \mathrm{E}-06$ & $9.83 \mathrm{E}-09$ & $5.30 \mathrm{E}-11$ & $4.63 \mathrm{E}-12$ & $7.48 \mathrm{E}-10$ \\
\hline $45 \%$ & $6.92 \mathrm{E}-03$ & $2.41 \mathrm{E}-05$ & $6.40 \mathrm{E}-03$ & $3.86 \mathrm{E}-04$ & $9.11 \mathrm{E}-06$ & $1.17 \mathrm{E}-06$ & $9.27 \mathrm{E}-06$ & $9.70 \mathrm{E}-08$ & $6.64 \mathrm{E}-06$ & $1.05 \mathrm{E}-08$ & $5.53 \mathrm{E}-11$ & $5.07 \mathrm{E}-12$ & 8.09E-10 \\
\hline $50 \%$ & $7.12 \mathrm{E}-03$ & $2.62 \mathrm{E}-05$ & $6.57 \mathrm{E}-03$ & $4.15 \mathrm{E}-04$ & $9.62 \mathrm{E}-06$ & $1.29 \mathrm{E}-06$ & $1.03 \mathrm{E}-05$ & $9.70 \mathrm{E}-08$ & $6.64 \mathrm{E}-06$ & $1.12 \mathrm{E}-08$ & $5.78 \mathrm{E}-11$ & $5.59 \mathrm{E}-12$ & $8.71 \mathrm{E}-10$ \\
\hline $55 \%$ & $7.31 \mathrm{E}-03$ & $2.78 \mathrm{E}-05$ & $6.72 \mathrm{E}-03$ & $4.50 \mathrm{E}-04$ & $1.01 \mathrm{E}-05$ & $1.45 \mathrm{E}-06$ & $1.14 \mathrm{E}-05$ & $9.70 \mathrm{E}-08$ & $6.64 \mathrm{E}-06$ & $1.19 \mathrm{E}-08$ & $6.03 \mathrm{E}-11$ & $6.10 \mathrm{E}-12$ & $9.31 \mathrm{E}-10$ \\
\hline $60 \%$ & 7.49E-03 & $2.93 \mathrm{E}-05$ & $6.88 \mathrm{E}-03$ & $4.88 \mathrm{E}-04$ & $1.06 \mathrm{E}-05$ & $1.61 \mathrm{E}-06$ & $1.27 \mathrm{E}-05$ & $9.70 \mathrm{E}-08$ & $6.64 \mathrm{E}-06$ & $1.27 \mathrm{E}-08$ & $6.32 \mathrm{E}-11$ & $6.76 \mathrm{E}-12$ & $9.95 \mathrm{E}-10$ \\
\hline $65 \%$ & $7.69 \mathrm{E}-03$ & $3.07 \mathrm{E}-05$ & 7.09E-03 & $5.27 \mathrm{E}-04$ & 1.10E-05 & 1.77E-06 & $1.40 \mathrm{E}-05$ & $9.70 \mathrm{E}-08$ & $6.64 \mathrm{E}-06$ & $1.35 \mathrm{E}-08$ & $6.62 \mathrm{E}-11$ & 7.40E-12 & $1.06 \mathrm{E}-09$ \\
\hline $70 \%$ & $7.95 \mathrm{E}-03$ & $3.22 \mathrm{E}-05$ & $7.30 \mathrm{E}-03$ & $5.64 \mathrm{E}-04$ & 1.14E-05 & $1.95 \mathrm{E}-06$ & $1.52 \mathrm{E}-05$ & $9.70 \mathrm{E}-08$ & $6.64 \mathrm{E}-06$ & $1.45 \mathrm{E}-08$ & $7.00 \mathrm{E}-11$ & $8.06 \mathrm{E}-12$ & $1.13 \mathrm{E}-09$ \\
\hline $75 \%$ & $8.26 \mathrm{E}-03$ & $3.33 \mathrm{E}-05$ & $7.58 \mathrm{E}-03$ & $6.18 \mathrm{E}-04$ & 1.19E-05 & $2.22 \mathrm{E}-06$ & $1.66 \mathrm{E}-05$ & $9.70 \mathrm{E}-08$ & $6.64 \mathrm{E}-06$ & $1.54 \mathrm{E}-08$ & 7.43E-11 & $9.02 \mathrm{E}-12$ & $1.20 \mathrm{E}-09$ \\
\hline $80 \%$ & $8.66 \mathrm{E}-03$ & $3.47 \mathrm{E}-05$ & 8.01E-03 & $6.75 \mathrm{E}-04$ & $1.24 \mathrm{E}-05$ & $2.50 \mathrm{E}-06$ & $1.81 \mathrm{E}-05$ & $9.70 \mathrm{E}-08$ & $6.64 \mathrm{E}-06$ & $1.67 \mathrm{E}-08$ & 7.91E-11 & $1.01 \mathrm{E}-11$ & $1.28 \mathrm{E}-09$ \\
\hline $85 \%$ & $9.24 \mathrm{E}-03$ & $3.61 \mathrm{E}-05$ & $8.59 \mathrm{E}-03$ & $7.46 \mathrm{E}-04$ & $1.30 \mathrm{E}-05$ & $2.89 \mathrm{E}-06$ & $1.99 \mathrm{E}-05$ & $9.70 \mathrm{E}-08$ & $6.64 \mathrm{E}-06$ & $1.86 \mathrm{E}-08$ & $8.56 \mathrm{E}-11$ & $1.16 \mathrm{E}-11$ & $1.39 \mathrm{E}-09$ \\
\hline $90 \%$ & $1.00 \mathrm{E}-02$ & $3.78 \mathrm{E}-05$ & $9.36 \mathrm{E}-03$ & 8.68E-04 & $1.38 \mathrm{E}-05$ & $3.42 \mathrm{E}-06$ & $2.22 \mathrm{E}-05$ & $9.70 \mathrm{E}-08$ & $6.64 \mathrm{E}-06$ & $2.12 \mathrm{E}-08$ & $9.50 \mathrm{E}-11$ & $1.37 \mathrm{E}-11$ & $1.50 \mathrm{E}-09$ \\
\hline $95 \%$ & $1.19 \mathrm{E}-02$ & $4.02 \mathrm{E}-05$ & $1.13 \mathrm{E}-02$ & $1.06 \mathrm{E}-03$ & $1.49 \mathrm{E}-05$ & $4.56 \mathrm{E}-06$ & $2.57 \mathrm{E}-05$ & $9.70 \mathrm{E}-08$ & $6.64 \mathrm{E}-06$ & $2.56 \mathrm{E}-08$ & $1.12 \mathrm{E}-10$ & $1.79 \mathrm{E}-11$ & 1.69E-09 \\
\hline Mean & $6.98 \mathrm{E}-03$ & $2.38 \mathrm{E}-05$ & $6.49 \mathrm{E}-03$ & $4.80 \mathrm{E}-04$ & $9.24 \mathrm{E}-06$ & $1.72 \mathrm{E}-06$ & $1.14 \mathrm{E}-05$ & $9.70 \mathrm{E}-08$ & $6.64 \mathrm{E}-06$ & $1.28 \mathrm{E}-08$ & $6.37 \mathrm{E}-11$ & $7.17 \mathrm{E}-12$ & $8.95 \mathrm{E}-10$ \\
\hline Min & $1.49 \mathrm{E}-03$ & $1.90 \mathrm{E}-07$ & $1.42 \mathrm{E}-03$ & $3.37 \mathrm{E}-05$ & $1.64 \mathrm{E}-06$ & 5.33E-08 & 4.81E-08 & $9.46 \mathrm{E}-08$ & $6.47 \mathrm{E}-06$ & $1.97 \mathrm{E}-09$ & $2.27 \mathrm{E}-11$ & $6.44 \mathrm{E}-13$ & $1.13 \mathrm{E}-11$ \\
\hline Max & $1.08 \mathrm{E}-01$ & $4.98 \mathrm{E}-05$ & $1.08 \mathrm{E}-01$ & $3.70 \mathrm{E}-03$ & $2.14 \mathrm{E}-05$ & $1.26 \mathrm{E}-05$ & $3.97 \mathrm{E}-05$ & $9.70 \mathrm{E}-08$ & $6.64 \mathrm{E}-06$ & 2.13E-07 & $3.07 \mathrm{E}-10$ & 7.49E-11 & 2.43E-09 \\
\hline
\end{tabular}




\section{TABLE C.10 Peak DSR Percentiles (mrem/yr per pCi/g) for Different Exposure Pathways for Ba-133 in Offsite Resident Scenario}

\begin{tabular}{|c|c|c|c|c|c|c|c|c|c|c|c|c|c|}
\hline Percentile & Total & External-WD & Fish & Plant-WD & Meat-WD & Milk-WD & Soil-WD & External-WI & Inhalation & Plant-WI & Meat-WI & Milk-WI & Soil-WI \\
\hline $5 \%$ & $6.66 \mathrm{E}-05$ & $1.17 \mathrm{E}-05$ & $3.91 \mathrm{E}-06$ & $1.17 \mathrm{E}-06$ & $5.06 \mathrm{E}-08$ & $6.46 \mathrm{E}-07$ & $5.64 \mathrm{E}-10$ & $6.15 \mathrm{E}-05$ & $6.52 \mathrm{E}-10$ & $3.29 \mathrm{E}-11$ & $2.41 \mathrm{E}-13$ & $2.64 \mathrm{E}-12$ & $4.45 \mathrm{E}-14$ \\
\hline $10 \%$ & $7.01 \mathrm{E}-05$ & $1.73 \mathrm{E}-05$ & $5.54 \mathrm{E}-06$ & $1.48 \mathrm{E}-06$ & $7.05 \mathrm{E}-08$ & 8.64E-07 & $8.27 \mathrm{E}-10$ & $6.26 \mathrm{E}-05$ & $6.64 \mathrm{E}-10$ & $3.80 \mathrm{E}-11$ & $3.40 \mathrm{E}-13$ & $3.40 \mathrm{E}-12$ & $6.42 \mathrm{E}-14$ \\
\hline $15 \%$ & 7.37E-05 & $2.23 \mathrm{E}-05$ & $6.49 \mathrm{E}-06$ & $1.68 \mathrm{E}-06$ & 8.99E-08 & $1.05 \mathrm{E}-06$ & $1.08 \mathrm{E}-09$ & $6.29 \mathrm{E}-05$ & $6.67 \mathrm{E}-10$ & $4.27 \mathrm{E}-11$ & $4.20 \mathrm{E}-13$ & $4.03 \mathrm{E}-12$ & $8.32 \mathrm{E}-14$ \\
\hline $20 \%$ & 7.64E-05 & $2.68 \mathrm{E}-05$ & $7.15 \mathrm{E}-06$ & $1.87 \mathrm{E}-06$ & $1.07 \mathrm{E}-07$ & $1.20 \mathrm{E}-06$ & $1.34 \mathrm{E}-09$ & $6.30 \mathrm{E}-05$ & $6.69 \mathrm{E}-10$ & $4.74 \mathrm{E}-11$ & $5.01 \mathrm{E}-13$ & $4.60 \mathrm{E}-12$ & $1.01 \mathrm{E}-13$ \\
\hline $25 \%$ & $7.84 \mathrm{E}-05$ & $3.03 \mathrm{E}-05$ & 7.44E-06 & $2.05 \mathrm{E}-06$ & $1.26 \mathrm{E}-07$ & $1.38 \mathrm{E}-06$ & $1.58 \mathrm{E}-09$ & $6.31 \mathrm{E}-05$ & $6.70 \mathrm{E}-10$ & $5.10 \mathrm{E}-11$ & $5.87 \mathrm{E}-13$ & $5.18 \mathrm{E}-12$ & $1.17 \mathrm{E}-13$ \\
\hline $30 \%$ & $8.00 \mathrm{E}-05$ & $3.30 \mathrm{E}-05$ & $7.56 \mathrm{E}-06$ & $2.22 \mathrm{E}-06$ & $1.47 \mathrm{E}-07$ & $1.54 \mathrm{E}-06$ & $1.83 \mathrm{E}-09$ & $6.31 \mathrm{E}-05$ & $6.70 \mathrm{E}-10$ & $5.51 \mathrm{E}-11$ & $6.74 \mathrm{E}-13$ & $5.72 \mathrm{E}-12$ & $1.30 \mathrm{E}-13$ \\
\hline $35 \%$ & $8.12 \mathrm{E}-05$ & 3.53E-05 & $7.65 \mathrm{E}-06$ & $2.39 \mathrm{E}-06$ & $1.68 \mathrm{E}-07$ & $1.70 \mathrm{E}-06$ & $2.05 \mathrm{E}-09$ & $6.31 \mathrm{E}-05$ & $6.70 \mathrm{E}-10$ & $5.91 \mathrm{E}-11$ & $7.70 \mathrm{E}-13$ & $6.32 \mathrm{E}-12$ & $1.44 \mathrm{E}-13$ \\
\hline $40 \%$ & $8.22 \mathrm{E}-05$ & $3.70 \mathrm{E}-05$ & $7.75 \mathrm{E}-06$ & $2.57 \mathrm{E}-06$ & $1.90 \mathrm{E}-07$ & $1.87 \mathrm{E}-06$ & $2.27 \mathrm{E}-09$ & $6.32 \mathrm{E}-05$ & $6.70 \mathrm{E}-10$ & $6.33 \mathrm{E}-11$ & $8.68 \mathrm{E}-13$ & $6.91 \mathrm{E}-12$ & $1.58 \mathrm{E}-13$ \\
\hline $45 \%$ & $8.31 \mathrm{E}-05$ & $3.85 \mathrm{E}-05$ & $7.85 \mathrm{E}-06$ & $2.75 \mathrm{E}-06$ & $2.15 \mathrm{E}-07$ & $2.06 \mathrm{E}-06$ & 2.49E-09 & $6.32 \mathrm{E}-05$ & $6.70 \mathrm{E}-10$ & $6.77 \mathrm{E}-11$ & $9.75 \mathrm{E}-13$ & $7.55 \mathrm{E}-12$ & $1.71 \mathrm{E}-13$ \\
\hline $50 \%$ & $8.41 \mathrm{E}-05$ & $3.98 \mathrm{E}-05$ & 7.97E-06 & $2.94 \mathrm{E}-06$ & $2.41 \mathrm{E}-07$ & $2.27 \mathrm{E}-06$ & $2.71 \mathrm{E}-09$ & $6.32 \mathrm{E}-05$ & $6.70 \mathrm{E}-10$ & $7.24 \mathrm{E}-11$ & $1.10 \mathrm{E}-12$ & $8.22 \mathrm{E}-12$ & $1.85 \mathrm{E}-13$ \\
\hline $55 \%$ & 8.49E-05 & $4.10 \mathrm{E}-05$ & 8.08E-06 & $3.16 \mathrm{E}-06$ & $2.74 \mathrm{E}-07$ & $2.49 \mathrm{E}-06$ & $2.91 \mathrm{E}-09$ & $6.32 \mathrm{E}-05$ & $6.70 \mathrm{E}-10$ & $7.72 \mathrm{E}-11$ & $1.23 \mathrm{E}-12$ & 8.99E-12 & $1.99 \mathrm{E}-13$ \\
\hline $60 \%$ & $8.58 \mathrm{E}-05$ & $4.21 \mathrm{E}-05$ & $8.23 \mathrm{E}-06$ & $3.37 \mathrm{E}-06$ & $3.09 \mathrm{E}-07$ & $2.75 \mathrm{E}-06$ & $3.16 \mathrm{E}-09$ & $6.32 \mathrm{E}-05$ & $6.70 \mathrm{E}-10$ & $8.19 \mathrm{E}-11$ & $1.38 \mathrm{E}-12$ & $9.79 \mathrm{E}-12$ & $2.11 \mathrm{E}-13$ \\
\hline $65 \%$ & $8.68 \mathrm{E}-05$ & $4.31 \mathrm{E}-05$ & $8.40 \mathrm{E}-06$ & $3.61 \mathrm{E}-06$ & $3.50 \mathrm{E}-07$ & $3.03 \mathrm{E}-06$ & $3.38 \mathrm{E}-09$ & $6.32 \mathrm{E}-05$ & $6.70 \mathrm{E}-10$ & $8.69 \mathrm{E}-11$ & $1.56 \mathrm{E}-12$ & $1.07 \mathrm{E}-11$ & $2.25 \mathrm{E}-13$ \\
\hline $70 \%$ & $8.78 \mathrm{E}-05$ & 4.43E-05 & $8.61 \mathrm{E}-06$ & $3.88 \mathrm{E}-06$ & $4.01 \mathrm{E}-07$ & $3.34 \mathrm{E}-06$ & $3.62 \mathrm{E}-09$ & $6.32 \mathrm{E}-05$ & $6.70 \mathrm{E}-10$ & $9.35 \mathrm{E}-11$ & $1.78 \mathrm{E}-12$ & $1.18 \mathrm{E}-11$ & $2.38 \mathrm{E}-13$ \\
\hline $75 \%$ & $8.87 \mathrm{E}-05$ & $4.55 \mathrm{E}-05$ & $8.86 \mathrm{E}-06$ & $4.18 \mathrm{E}-06$ & $4.59 \mathrm{E}-07$ & $3.73 \mathrm{E}-06$ & $3.90 \mathrm{E}-09$ & $6.32 \mathrm{E}-05$ & $6.70 \mathrm{E}-10$ & $1.01 \mathrm{E}-10$ & $2.05 \mathrm{E}-12$ & $1.31 \mathrm{E}-11$ & $2.55 \mathrm{E}-13$ \\
\hline $80 \%$ & 8.99E-05 & 4.69E-05 & $9.20 \mathrm{E}-06$ & 4.54E-06 & $5.33 \mathrm{E}-07$ & $4.16 \mathrm{E}-06$ & 4.19E-09 & $6.32 \mathrm{E}-05$ & $6.70 \mathrm{E}-10$ & $1.09 \mathrm{E}-10$ & $2.38 \mathrm{E}-12$ & $1.47 \mathrm{E}-11$ & $2.73 \mathrm{E}-13$ \\
\hline $85 \%$ & $9.12 \mathrm{E}-05$ & 4.84E-05 & $9.67 \mathrm{E}-06$ & 4.97E-06 & $6.49 \mathrm{E}-07$ & $4.76 \mathrm{E}-06$ & 4.54E-09 & $6.32 \mathrm{E}-05$ & $6.70 \mathrm{E}-10$ & $1.20 \mathrm{E}-10$ & $2.85 \mathrm{E}-12$ & $1.68 \mathrm{E}-11$ & $2.94 \mathrm{E}-13$ \\
\hline $90 \%$ & $9.30 \mathrm{E}-05$ & $5.04 \mathrm{E}-05$ & $1.05 \mathrm{E}-05$ & $5.60 \mathrm{E}-06$ & $8.00 \mathrm{E}-07$ & $5.63 \mathrm{E}-06$ & $5.05 \mathrm{E}-09$ & $6.32 \mathrm{E}-05$ & $6.70 \mathrm{E}-10$ & $1.37 \mathrm{E}-10$ & $3.60 \mathrm{E}-12$ & $1.98 \mathrm{E}-11$ & $3.23 \mathrm{E}-13$ \\
\hline $95 \%$ & $9.59 \mathrm{E}-05$ & $5.32 \mathrm{E}-05$ & $1.23 \mathrm{E}-05$ & $6.60 \mathrm{E}-06$ & $1.12 \mathrm{E}-06$ & $7.40 \mathrm{E}-06$ & $5.76 \mathrm{E}-09$ & $6.32 \mathrm{E}-05$ & $6.70 \mathrm{E}-10$ & $1.64 \mathrm{E}-10$ & $4.98 \mathrm{E}-12$ & $2.55 \mathrm{E}-11$ & $3.61 \mathrm{E}-13$ \\
\hline Mean & $8.32 \mathrm{E}-05$ & $3.70 \mathrm{E}-05$ & $8.21 \mathrm{E}-06$ & $3.32 \mathrm{E}-06$ & $3.72 \mathrm{E}-07$ & $2.91 \mathrm{E}-06$ & $2.88 \mathrm{E}-09$ & $6.28 \mathrm{E}-05$ & $6.66 \mathrm{E}-10$ & $8.21 \mathrm{E}-11$ & $1.67 \mathrm{E}-12$ & $1.04 \mathrm{E}-11$ & $1.91 \mathrm{E}-13$ \\
\hline Min & $4.88 \mathrm{E}-05$ & $2.07 \mathrm{E}-06$ & $1.92 \mathrm{E}-06$ & $3.07 \mathrm{E}-07$ & $5.42 \mathrm{E}-09$ & $1.67 \mathrm{E}-07$ & $2.96 \mathrm{E}-11$ & $4.32 \mathrm{E}-05$ & $4.58 \mathrm{E}-10$ & $1.17 \mathrm{E}-11$ & $6.53 \mathrm{E}-14$ & $1.02 \mathrm{E}-12$ & $3.77 \mathrm{E}-15$ \\
\hline Max & $4.40 \mathrm{E}-04$ & $3.61 \mathrm{E}-04$ & $9.33 \mathrm{E}-05$ & $1.83 \mathrm{E}-05$ & $4.10 \mathrm{E}-06$ & $2.09 \mathrm{E}-05$ & 4.70E-08 & $6.32 \mathrm{E}-05$ & $6.70 \mathrm{E}-10$ & $9.38 \mathrm{E}-10$ & $2.13 \mathrm{E}-11$ & $7.58 \mathrm{E}-11$ & $1.13 \mathrm{E}-12$ \\
\hline
\end{tabular}




\section{TABLE C.11 Peak DSR Percentiles (mrem/yr per pCi/g) for Different Exposure Pathways for C-14 in Offsite Resident Scenario}

\begin{tabular}{|c|c|c|c|c|c|c|c|c|c|c|c|c|c|}
\hline Percentile & Total & External-WD & Fish & Plant-WD & Meat-WD & Milk-WD & Soil-WD & External-WI & Inhalation & Plant-WI & Meat-WI & Milk-WI & Soil-WI \\
\hline $5 \%$ & $2.48 \mathrm{E}-04$ & $4.57 \mathrm{E}-12$ & $2.43 \mathrm{E}-04$ & $4.02 \mathrm{E}-07$ & 4.44E-07 & $1.03 \mathrm{E}-06$ & $1.40 \mathrm{E}-11$ & $1.27 \mathrm{E}-15$ & $9.49 \mathrm{E}-07$ & $2.40 \mathrm{E}-11$ & $1.54 \mathrm{E}-11$ & $2.95 \mathrm{E}-11$ & $6.03 \mathrm{E}-16$ \\
\hline $10 \%$ & $3.76 \mathrm{E}-04$ & $6.03 \mathrm{E}-12$ & $3.66 \mathrm{E}-04$ & $5.31 \mathrm{E}-07$ & $5.01 \mathrm{E}-07$ & $1.06 \mathrm{E}-06$ & $2.03 \mathrm{E}-11$ & $1.31 \mathrm{E}-15$ & $9.95 \mathrm{E}-07$ & $3.04 \mathrm{E}-11$ & $1.68 \mathrm{E}-11$ & $3.07 \mathrm{E}-11$ & 8.64E-16 \\
\hline $15 \%$ & 4.97E-04 & $7.71 \mathrm{E}-12$ & $4.86 \mathrm{E}-04$ & $7.40 \mathrm{E}-07$ & $5.82 \mathrm{E}-07$ & $1.09 \mathrm{E}-06$ & $2.71 \mathrm{E}-11$ & $1.34 \mathrm{E}-15$ & $1.03 \mathrm{E}-06$ & $3.72 \mathrm{E}-11$ & $1.82 \mathrm{E}-11$ & $3.15 \mathrm{E}-11$ & $1.14 \mathrm{E}-15$ \\
\hline $20 \%$ & $6.40 \mathrm{E}-04$ & $9.77 \mathrm{E}-12$ & $6.27 \mathrm{E}-04$ & $1.04 \mathrm{E}-06$ & $6.71 \mathrm{E}-07$ & $1.12 \mathrm{E}-06$ & $3.53 \mathrm{E}-11$ & $1.38 \mathrm{E}-15$ & $1.05 \mathrm{E}-06$ & 4.49E-11 & $1.97 \mathrm{E}-11$ & $3.23 \mathrm{E}-11$ & $1.38 \mathrm{E}-15$ \\
\hline $25 \%$ & 8.00E-04 & $1.19 \mathrm{E}-11$ & 7.79E-04 & $1.36 \mathrm{E}-06$ & 7.97E-07 & $1.15 \mathrm{E}-06$ & $4.42 \mathrm{E}-11$ & $1.42 \mathrm{E}-15$ & $1.07 \mathrm{E}-06$ & $5.40 \mathrm{E}-11$ & $2.14 \mathrm{E}-11$ & $3.29 \mathrm{E}-11$ & $1.61 \mathrm{E}-15$ \\
\hline $30 \%$ & $9.86 \mathrm{E}-04$ & $1.38 \mathrm{E}-11$ & $9.68 \mathrm{E}-04$ & $1.71 \mathrm{E}-06$ & $9.40 \mathrm{E}-07$ & $1.18 \mathrm{E}-06$ & $5.63 \mathrm{E}-11$ & $1.47 \mathrm{E}-15$ & $1.09 \mathrm{E}-06$ & $6.51 \mathrm{E}-11$ & $2.33 \mathrm{E}-11$ & $3.36 \mathrm{E}-11$ & $1.91 \mathrm{E}-15$ \\
\hline $35 \%$ & $1.21 \mathrm{E}-03$ & $1.57 \mathrm{E}-11$ & 1.19E-03 & $2.07 \mathrm{E}-06$ & $1.15 \mathrm{E}-06$ & $1.23 \mathrm{E}-06$ & $6.97 \mathrm{E}-11$ & $1.52 \mathrm{E}-15$ & $1.11 \mathrm{E}-06$ & $8.00 \mathrm{E}-11$ & $2.53 \mathrm{E}-11$ & $3.43 \mathrm{E}-11$ & $2.25 \mathrm{E}-15$ \\
\hline $40 \%$ & $1.47 \mathrm{E}-03$ & $1.75 \mathrm{E}-11$ & $1.44 \mathrm{E}-03$ & $2.55 \mathrm{E}-06$ & $1.40 \mathrm{E}-06$ & $1.29 \mathrm{E}-06$ & $8.51 \mathrm{E}-11$ & $1.57 \mathrm{E}-15$ & $1.12 \mathrm{E}-06$ & $9.45 \mathrm{E}-11$ & 2.76E-11 & $3.50 \mathrm{E}-11$ & 2.61E-15 \\
\hline $45 \%$ & $1.85 \mathrm{E}-03$ & $2.04 \mathrm{E}-11$ & $1.80 \mathrm{E}-03$ & $3.21 \mathrm{E}-06$ & $1.70 \mathrm{E}-06$ & $1.39 \mathrm{E}-06$ & $1.06 \mathrm{E}-10$ & $1.63 \mathrm{E}-15$ & $1.14 \mathrm{E}-06$ & $1.08 \mathrm{E}-10$ & 3.03E-11 & $3.56 \mathrm{E}-11$ & $2.97 \mathrm{E}-15$ \\
\hline $50 \%$ & $2.36 \mathrm{E}-03$ & 3.00E-11 & $2.32 \mathrm{E}-03$ & $4.26 \mathrm{E}-06$ & 2.32E-06 & $1.69 \mathrm{E}-06$ & $1.40 \mathrm{E}-10$ & $1.66 \mathrm{E}-15$ & $1.16 \mathrm{E}-06$ & 1.19E-10 & 3.32E-11 & 3.63E-11 & $3.39 \mathrm{E}-15$ \\
\hline $55 \%$ & $2.96 \mathrm{E}-03$ & $6.93 \mathrm{E}-11$ & 2.89E-03 & $6.15 \mathrm{E}-06$ & $3.42 \mathrm{E}-06$ & $2.99 \mathrm{E}-06$ & $2.55 \mathrm{E}-10$ & $1.69 \mathrm{E}-15$ & $1.17 \mathrm{E}-06$ & $1.34 \mathrm{E}-10$ & $3.63 \mathrm{E}-11$ & $3.71 \mathrm{E}-11$ & $3.86 \mathrm{E}-15$ \\
\hline $60 \%$ & $3.89 \mathrm{E}-03$ & $1.46 \mathrm{E}-10$ & $3.79 \mathrm{E}-03$ & $1.07 \mathrm{E}-05$ & $6.52 \mathrm{E}-06$ & $5.74 \mathrm{E}-06$ & $5.35 \mathrm{E}-10$ & $1.72 \mathrm{E}-15$ & $1.19 \mathrm{E}-06$ & $1.49 \mathrm{E}-10$ & $3.99 \mathrm{E}-11$ & 3.79E-11 & 4.37E-15 \\
\hline $65 \%$ & $5.35 \mathrm{E}-03$ & $2.68 \mathrm{E}-10$ & 5.19E-03 & $1.94 \mathrm{E}-05$ & $1.21 \mathrm{E}-05$ & $1.16 \mathrm{E}-05$ & 1.07E-09 & $1.74 \mathrm{E}-15$ & $1.20 \mathrm{E}-06$ & $1.65 \mathrm{E}-10$ & $4.42 \mathrm{E}-11$ & $3.87 \mathrm{E}-11$ & $4.88 \mathrm{E}-15$ \\
\hline $70 \%$ & 7.94E-03 & $5.22 \mathrm{E}-10$ & $7.56 \mathrm{E}-03$ & $3.54 \mathrm{E}-05$ & $2.12 \mathrm{E}-05$ & $2.25 \mathrm{E}-05$ & $2.06 \mathrm{E}-09$ & $1.77 \mathrm{E}-15$ & $1.22 \mathrm{E}-06$ & $1.84 \mathrm{E}-10$ & $4.92 \mathrm{E}-11$ & $3.97 \mathrm{E}-11$ & $5.42 \mathrm{E}-15$ \\
\hline $75 \%$ & $1.27 \mathrm{E}-02$ & $9.98 \mathrm{E}-10$ & $1.23 \mathrm{E}-02$ & $7.13 \mathrm{E}-05$ & $3.86 \mathrm{E}-05$ & $4.26 \mathrm{E}-05$ & $3.76 \mathrm{E}-09$ & $1.79 \mathrm{E}-15$ & $1.24 \mathrm{E}-06$ & $2.11 \mathrm{E}-10$ & $5.44 \mathrm{E}-11$ & 4.09E-11 & $5.97 \mathrm{E}-15$ \\
\hline $80 \%$ & $2.31 \mathrm{E}-02$ & $1.74 \mathrm{E}-09$ & $2.24 \mathrm{E}-02$ & $1.37 \mathrm{E}-04$ & $7.48 \mathrm{E}-05$ & $7.08 \mathrm{E}-05$ & 6.97E-09 & $1.82 \mathrm{E}-15$ & $1.26 \mathrm{E}-06$ & $2.39 \mathrm{E}-10$ & $6.16 \mathrm{E}-11$ & $4.26 \mathrm{E}-11$ & $6.60 \mathrm{E}-15$ \\
\hline $85 \%$ & 4.19E-02 & $3.28 \mathrm{E}-09$ & $4.00 \mathrm{E}-02$ & 3.03E-04 & $1.62 \mathrm{E}-04$ & $1.33 \mathrm{E}-04$ & $1.30 \mathrm{E}-08$ & $1.85 \mathrm{E}-15$ & $1.29 \mathrm{E}-06$ & $2.76 \mathrm{E}-10$ & $7.10 \mathrm{E}-11$ & 4.45E-11 & 7.32E-15 \\
\hline $90 \%$ & 8.49E-02 & $6.40 \mathrm{E}-09$ & 8.30E-02 & $6.75 \mathrm{E}-04$ & $3.80 \mathrm{E}-04$ & $2.92 \mathrm{E}-04$ & $2.53 \mathrm{E}-08$ & $1.88 \mathrm{E}-15$ & $1.32 \mathrm{E}-06$ & $3.42 \mathrm{E}-10$ & $8.41 \mathrm{E}-11$ & $4.76 \mathrm{E}-11$ & $8.23 \mathrm{E}-15$ \\
\hline $95 \%$ & 2.27E-01 & $1.40 \mathrm{E}-08$ & $2.26 \mathrm{E}-01$ & $2.26 \mathrm{E}-03$ & $1.20 \mathrm{E}-03$ & $6.93 \mathrm{E}-04$ & $5.96 \mathrm{E}-08$ & $1.94 \mathrm{E}-15$ & $1.36 \mathrm{E}-06$ & 4.79E-10 & $1.11 \mathrm{E}-10$ & $5.36 \mathrm{E}-11$ & $9.64 \mathrm{E}-15$ \\
\hline Mean & $5.95 \mathrm{E}-02$ & $2.68 \mathrm{E}-09$ & $5.89 \mathrm{E}-02$ & $4.88 \mathrm{E}-04$ & $2.43 \mathrm{E}-04$ & $1.21 \mathrm{E}-04$ & $1.16 \mathrm{E}-08$ & $1.62 \mathrm{E}-15$ & $1.16 \mathrm{E}-06$ & $1.68 \mathrm{E}-10$ & $4.59 \mathrm{E}-11$ & $3.93 \mathrm{E}-11$ & $4.09 \mathrm{E}-15$ \\
\hline Min & 4.91E-05 & $2.14 \mathrm{E}-12$ & $4.66 \mathrm{E}-05$ & $1.92 \mathrm{E}-07$ & $3.40 \mathrm{E}-07$ & $9.64 \mathrm{E}-07$ & $4.29 \mathrm{E}-13$ & $1.16 \mathrm{E}-15$ & 7.82E-07 & $9.76 \mathrm{E}-12$ & $1.22 \mathrm{E}-11$ & $2.74 \mathrm{E}-11$ & $1.81 \mathrm{E}-17$ \\
\hline Max & $9.51 \mathrm{E}+00$ & $1.51 \mathrm{E}-07$ & $9.51 \mathrm{E}+00$ & $5.68 \mathrm{E}-02$ & $3.86 \mathrm{E}-02$ & $9.22 \mathrm{E}-03$ & $6.30 \mathrm{E}-07$ & $4.26 \mathrm{E}-15$ & $1.55 \mathrm{E}-06$ & $5.22 \mathrm{E}-09$ & $1.39 \mathrm{E}-09$ & $1.76 \mathrm{E}-09$ & $8.85 \mathrm{E}-14$ \\
\hline
\end{tabular}




\section{TABLE C.12 Peak DSR Percentiles (mrem/yr per pCi/g) for Different Exposure Pathways for Cs-137 in Offsite Resident Scenario}

\begin{tabular}{|c|c|c|c|c|c|c|c|c|c|c|c|c|c|}
\hline Percentile & Total & External-WD & Fish & Plant-WD & Meat-WD & Milk-WD & Soil-WD & External-WI & Inhalation & Plant-WI & Meat-WI & Milk-WI & Soil-WI \\
\hline $5 \%$ & $2.69 \mathrm{E}-03$ & $1.81 \mathrm{E}-04$ & $1.31 \mathrm{E}-03$ & $1.72 \mathrm{E}-05$ & $3.22 \mathrm{E}-04$ & $2.16 \mathrm{E}-04$ & $2.45 \mathrm{E}-08$ & $1.76 \mathrm{E}-04$ & 2.67E-09 & $4.75 \mathrm{E}-10$ & $1.47 \mathrm{E}-09$ & $7.21 \mathrm{E}-10$ & $1.61 \mathrm{E}-12$ \\
\hline $10 \%$ & $3.10 \mathrm{E}-03$ & $2.10 \mathrm{E}-04$ & $1.71 \mathrm{E}-03$ & $2.08 \mathrm{E}-05$ & $3.76 \mathrm{E}-04$ & $2.56 \mathrm{E}-04$ & $3.48 \mathrm{E}-08$ & $1.76 \mathrm{E}-04$ & 2.67E-09 & $5.72 \mathrm{E}-10$ & $1.72 \mathrm{E}-09$ & $8.52 \mathrm{E}-10$ & $2.27 \mathrm{E}-12$ \\
\hline $15 \%$ & $3.45 \mathrm{E}-03$ & $2.22 \mathrm{E}-04$ & $2.03 \mathrm{E}-03$ & $2.34 \mathrm{E}-05$ & $4.18 \mathrm{E}-04$ & $2.88 \mathrm{E}-04$ & $4.39 \mathrm{E}-08$ & $1.76 \mathrm{E}-04$ & 2.67E-09 & $6.43 \mathrm{E}-10$ & $1.94 \mathrm{E}-09$ & $9.60 \mathrm{E}-10$ & $2.84 \mathrm{E}-12$ \\
\hline $20 \%$ & $3.76 \mathrm{E}-03$ & $2.32 \mathrm{E}-04$ & $2.31 \mathrm{E}-03$ & $2.57 \mathrm{E}-05$ & $4.56 \mathrm{E}-04$ & $3.17 \mathrm{E}-04$ & $5.04 \mathrm{E}-08$ & $1.76 \mathrm{E}-04$ & 2.67E-09 & $7.22 \mathrm{E}-10$ & 2.14E-09 & $1.06 \mathrm{E}-09$ & $3.27 \mathrm{E}-12$ \\
\hline $25 \%$ & $4.08 \mathrm{E}-03$ & $2.38 \mathrm{E}-04$ & $2.62 \mathrm{E}-03$ & $2.77 \mathrm{E}-05$ & 4.91E-04 & $3.44 \mathrm{E}-04$ & $5.70 \mathrm{E}-08$ & $1.76 \mathrm{E}-04$ & 2.67E-09 & $7.98 \mathrm{E}-10$ & 2.32E-09 & $1.16 \mathrm{E}-09$ & $3.66 \mathrm{E}-12$ \\
\hline $30 \%$ & $4.39 \mathrm{E}-03$ & $2.44 \mathrm{E}-04$ & $2.90 \mathrm{E}-03$ & $2.97 \mathrm{E}-05$ & $5.25 \mathrm{E}-04$ & $3.70 \mathrm{E}-04$ & $6.26 \mathrm{E}-08$ & $1.76 \mathrm{E}-04$ & 2.67E-09 & $8.74 \mathrm{E}-10$ & $2.50 \mathrm{E}-09$ & $1.24 \mathrm{E}-09$ & $3.99 \mathrm{E}-12$ \\
\hline $35 \%$ & $4.71 \mathrm{E}-03$ & 2.49E-04 & $3.19 \mathrm{E}-03$ & 3.19E-05 & $5.54 \mathrm{E}-04$ & $3.97 \mathrm{E}-04$ & $6.81 \mathrm{E}-08$ & $1.76 \mathrm{E}-04$ & 2.67E-09 & $9.42 \mathrm{E}-10$ & $2.70 \mathrm{E}-09$ & $1.33 \mathrm{E}-09$ & $4.31 \mathrm{E}-12$ \\
\hline $40 \%$ & $5.05 \mathrm{E}-03$ & $2.54 \mathrm{E}-04$ & $3.50 \mathrm{E}-03$ & $3.38 \mathrm{E}-05$ & $5.87 \mathrm{E}-04$ & $4.22 \mathrm{E}-04$ & 7.31E-08 & $1.76 \mathrm{E}-04$ & 2.67E-09 & $1.03 \mathrm{E}-09$ & 2.92E-09 & $1.42 \mathrm{E}-09$ & $4.59 \mathrm{E}-12$ \\
\hline $45 \%$ & $5.41 \mathrm{E}-03$ & $2.58 \mathrm{E}-04$ & $3.87 \mathrm{E}-03$ & $3.61 \mathrm{E}-05$ & $6.22 \mathrm{E}-04$ & $4.51 \mathrm{E}-04$ & $7.80 \mathrm{E}-08$ & $1.76 \mathrm{E}-04$ & 2.67E-09 & $1.10 \mathrm{E}-09$ & $3.14 \mathrm{E}-09$ & $1.52 \mathrm{E}-09$ & $4.88 \mathrm{E}-12$ \\
\hline $50 \%$ & $5.76 \mathrm{E}-03$ & 2.62E-04 & $4.21 \mathrm{E}-03$ & $3.86 \mathrm{E}-05$ & $6.55 \mathrm{E}-04$ & $4.75 \mathrm{E}-04$ & $8.29 \mathrm{E}-08$ & $1.76 \mathrm{E}-04$ & 2.67E-09 & 1.19E-09 & 3.37E-09 & $1.62 \mathrm{E}-09$ & $5.16 \mathrm{E}-12$ \\
\hline $55 \%$ & $6.13 \mathrm{E}-03$ & $2.66 \mathrm{E}-04$ & $4.60 \mathrm{E}-03$ & $4.11 \mathrm{E}-05$ & $6.92 \mathrm{E}-04$ & $5.07 \mathrm{E}-04$ & $8.75 \mathrm{E}-08$ & $1.76 \mathrm{E}-04$ & 2.67E-09 & $1.31 \mathrm{E}-09$ & $3.63 \mathrm{E}-09$ & $1.74 \mathrm{E}-09$ & $5.48 \mathrm{E}-12$ \\
\hline $60 \%$ & $6.54 \mathrm{E}-03$ & $2.70 \mathrm{E}-04$ & $5.04 \mathrm{E}-03$ & 4.41E-05 & $7.28 \mathrm{E}-04$ & $5.40 \mathrm{E}-04$ & $9.25 \mathrm{E}-08$ & $1.76 \mathrm{E}-04$ & $2.67 \mathrm{E}-09$ & $1.42 \mathrm{E}-09$ & $3.94 \mathrm{E}-09$ & $1.86 \mathrm{E}-09$ & $5.75 \mathrm{E}-12$ \\
\hline $65 \%$ & 7.07E-03 & $2.75 \mathrm{E}-04$ & $5.50 \mathrm{E}-03$ & $4.70 \mathrm{E}-05$ & 7.73E-04 & $5.73 \mathrm{E}-04$ & $9.84 \mathrm{E}-08$ & $1.76 \mathrm{E}-04$ & 2.67E-09 & $1.57 \mathrm{E}-09$ & $4.25 \mathrm{E}-09$ & $1.99 \mathrm{E}-09$ & $6.04 \mathrm{E}-12$ \\
\hline $70 \%$ & $7.62 \mathrm{E}-03$ & $2.79 \mathrm{E}-04$ & $6.08 \mathrm{E}-03$ & $5.09 \mathrm{E}-05$ & $8.19 \mathrm{E}-04$ & $6.15 \mathrm{E}-04$ & $1.04 \mathrm{E}-07$ & $1.76 \mathrm{E}-04$ & $2.67 \mathrm{E}-09$ & $1.72 \mathrm{E}-09$ & $4.63 \mathrm{E}-09$ & $2.15 \mathrm{E}-09$ & $6.42 \mathrm{E}-12$ \\
\hline $75 \%$ & $8.38 \mathrm{E}-03$ & $2.84 \mathrm{E}-04$ & $6.75 \mathrm{E}-03$ & $5.56 \mathrm{E}-05$ & 8.73E-04 & $6.61 \mathrm{E}-04$ & $1.11 \mathrm{E}-07$ & $1.76 \mathrm{E}-04$ & 2.67E-09 & $1.94 \mathrm{E}-09$ & $5.08 \mathrm{E}-09$ & 2.33E-09 & $6.81 \mathrm{E}-12$ \\
\hline $80 \%$ & $9.14 \mathrm{E}-03$ & $2.90 \mathrm{E}-04$ & $7.60 \mathrm{E}-03$ & $6.12 \mathrm{E}-05$ & $9.39 \mathrm{E}-04$ & $7.20 \mathrm{E}-04$ & $1.18 \mathrm{E}-07$ & $1.76 \mathrm{E}-04$ & 2.67E-09 & $2.26 \mathrm{E}-09$ & $5.67 \mathrm{E}-09$ & $2.58 \mathrm{E}-09$ & $7.21 \mathrm{E}-12$ \\
\hline $85 \%$ & $1.03 \mathrm{E}-02$ & $2.97 \mathrm{E}-04$ & $8.70 \mathrm{E}-03$ & $6.83 \mathrm{E}-05$ & $1.02 \mathrm{E}-03$ & 7.90E-04 & $1.27 \mathrm{E}-07$ & $1.76 \mathrm{E}-04$ & 2.67E-09 & $2.75 \mathrm{E}-09$ & $6.57 \mathrm{E}-09$ & $2.87 \mathrm{E}-09$ & $7.73 \mathrm{E}-12$ \\
\hline $90 \%$ & $1.18 \mathrm{E}-02$ & $3.06 \mathrm{E}-04$ & $1.03 \mathrm{E}-02$ & $7.85 \mathrm{E}-05$ & $1.14 \mathrm{E}-03$ & $8.84 \mathrm{E}-04$ & $1.38 \mathrm{E}-07$ & $1.76 \mathrm{E}-04$ & 2.67E-09 & $3.54 \mathrm{E}-09$ & 8.06E-09 & 3.36E-09 & $8.42 \mathrm{E}-12$ \\
\hline $95 \%$ & $1.49 \mathrm{E}-02$ & $3.17 \mathrm{E}-04$ & $1.34 \mathrm{E}-02$ & $1.01 \mathrm{E}-04$ & $1.31 \mathrm{E}-03$ & $1.06 \mathrm{E}-03$ & $1.53 \mathrm{E}-07$ & $1.76 \mathrm{E}-04$ & 2.67E-09 & $5.16 \mathrm{E}-09$ & $1.14 \mathrm{E}-08$ & 4.31E-09 & $9.31 \mathrm{E}-12$ \\
\hline Mean & $6.87 \mathrm{E}-03$ & $2.58 \mathrm{E}-04$ & $5.38 \mathrm{E}-03$ & 4.74E-05 & 7.16E-04 & $5.35 \mathrm{E}-04$ & $8.56 \mathrm{E}-08$ & $1.76 \mathrm{E}-04$ & 2.67E-09 & $1.84 \mathrm{E}-09$ & $4.55 \mathrm{E}-09$ & $1.99 \mathrm{E}-09$ & $5.30 \mathrm{E}-12$ \\
\hline Min & $9.86 \mathrm{E}-04$ & $4.90 \mathrm{E}-05$ & $3.63 \mathrm{E}-04$ & $6.18 \mathrm{E}-06$ & $1.06 \mathrm{E}-04$ & 6.61E-05 & $1.36 \mathrm{E}-09$ & $1.75 \mathrm{E}-04$ & $2.65 \mathrm{E}-09$ & $2.00 \mathrm{E}-10$ & $7.65 \mathrm{E}-10$ & $3.55 \mathrm{E}-10$ & $8.30 \mathrm{E}-14$ \\
\hline $\operatorname{Max}$ & $5.79 \mathrm{E}-02$ & $3.91 \mathrm{E}-04$ & $5.56 \mathrm{E}-02$ & $8.40 \mathrm{E}-04$ & $2.60 \mathrm{E}-03$ & $2.20 \mathrm{E}-03$ & $3.60 \mathrm{E}-07$ & $1.76 \mathrm{E}-04$ & 2.67E-09 & $5.23 \mathrm{E}-08$ & $7.55 \mathrm{E}-08$ & $3.10 \mathrm{E}-08$ & $2.08 \mathrm{E}-11$ \\
\hline
\end{tabular}




\section{TABLE C.13 Peak DSR Percentiles (mrem/yr per pCi/g) for Different Exposure Pathways for Pu-238 in Offsite Resident Scenario}

\begin{tabular}{|c|c|c|c|c|c|c|c|c|c|c|c|c|c|c|c|}
\hline Percentile & Total & External-WD & Fish & Radon-WD & Plant-WD & Meat-WD & Milk-WD & Soil-WD & External-WI & Inhalation & Radon & Plant-WI & Meat-WI & Milk-WI & Soil-WI \\
\hline $5 \%$ & 8.84E-04 & $1.40 \mathrm{E}-08$ & $6.03 \mathrm{E}-04$ & $1.33 \mathrm{E}-10$ & $1.82 \mathrm{E}-04$ & $9.05 \mathrm{E}-06$ & $2.99 \mathrm{E}-07$ & 7.24E-07 & $1.11 \mathrm{E}-10$ & $7.58 \mathrm{E}-06$ & $2.25 \mathrm{E}-10$ & $4.95 \mathrm{E}-09$ & $6.17 \mathrm{E}-11$ & $1.26 \mathrm{E}-12$ & $7.45 \mathrm{E}-11$ \\
\hline $10 \%$ & $1.18 \mathrm{E}-03$ & $1.64 \mathrm{E}-08$ & 7.77E-04 & $3.25 \mathrm{E}-10$ & $2.39 \mathrm{E}-04$ & $1.24 \mathrm{E}-05$ & $3.75 \mathrm{E}-07$ & $1.11 \mathrm{E}-06$ & $1.11 \mathrm{E}-10$ & $7.58 \mathrm{E}-06$ & $5.65 \mathrm{E}-10$ & $5.85 \mathrm{E}-09$ & $6.64 \mathrm{E}-11$ & $1.47 \mathrm{E}-12$ & $1.09 \mathrm{E}-10$ \\
\hline $15 \%$ & $1.37 \mathrm{E}-03$ & $1.77 \mathrm{E}-08$ & $8.74 \mathrm{E}-04$ & $4.88 \mathrm{E}-10$ & $2.80 \mathrm{E}-04$ & $1.49 \mathrm{E}-05$ & $4.45 \mathrm{E}-07$ & $1.46 \mathrm{E}-06$ & $1.26 \mathrm{E}-10$ & $7.58 \mathrm{E}-06$ & $8.38 \mathrm{E}-10$ & $6.61 \mathrm{E}-09$ & $6.98 \mathrm{E}-11$ & $1.65 \mathrm{E}-12$ & $1.35 \mathrm{E}-10$ \\
\hline $20 \%$ & $1.51 \mathrm{E}-03$ & $1.86 \mathrm{E}-08$ & $9.73 \mathrm{E}-04$ & $6.19 \mathrm{E}-10$ & $3.16 \mathrm{E}-04$ & $1.68 \mathrm{E}-05$ & $5.05 \mathrm{E}-07$ & $1.85 \mathrm{E}-06$ & $1.62 \mathrm{E}-10$ & $7.58 \mathrm{E}-06$ & $1.07 \mathrm{E}-09$ & $7.28 \mathrm{E}-09$ & $7.27 \mathrm{E}-11$ & $1.81 \mathrm{E}-12$ & $1.55 \mathrm{E}-10$ \\
\hline $25 \%$ & $1.65 \mathrm{E}-03$ & $1.95 \mathrm{E}-08$ & $1.06 \mathrm{E}-03$ & $7.08 \mathrm{E}-10$ & $3.46 \mathrm{E}-04$ & $1.81 \mathrm{E}-05$ & $5.59 \mathrm{E}-07$ & 2.17E-06 & $1.88 \mathrm{E}-10$ & $7.58 \mathrm{E}-06$ & $1.21 \mathrm{E}-09$ & $7.85 \mathrm{E}-09$ & $7.52 \mathrm{E}-11$ & $1.95 \mathrm{E}-12$ & $1.76 \mathrm{E}-10$ \\
\hline $30 \%$ & $1.75 \mathrm{E}-03$ & 2.04E-08 & $1.17 \mathrm{E}-03$ & 7.74E-10 & $3.72 \mathrm{E}-04$ & $1.92 \mathrm{E}-05$ & $6.05 \mathrm{E}-07$ & $2.46 \mathrm{E}-06$ & $2.06 \mathrm{E}-10$ & $7.58 \mathrm{E}-06$ & $1.32 \mathrm{E}-09$ & 8.45E-09 & 7.74E-11 & $2.09 \mathrm{E}-12$ & $1.94 \mathrm{E}-10$ \\
\hline $35 \%$ & $1.88 \mathrm{E}-03$ & $2.12 \mathrm{E}-08$ & $1.27 \mathrm{E}-03$ & 8.17E-10 & $4.05 \mathrm{E}-04$ & $2.01 \mathrm{E}-05$ & $6.56 \mathrm{E}-07$ & $2.72 \mathrm{E}-06$ & $2.18 \mathrm{E}-10$ & $7.58 \mathrm{E}-06$ & $1.39 \mathrm{E}-09$ & $9.01 \mathrm{E}-09$ & 7.97E-11 & $2.23 \mathrm{E}-12$ & $2.09 \mathrm{E}-10$ \\
\hline $40 \%$ & $2.02 \mathrm{E}-03$ & $2.21 \mathrm{E}-08$ & $1.38 \mathrm{E}-03$ & $8.45 \mathrm{E}-10$ & $4.36 \mathrm{E}-04$ & $2.10 \mathrm{E}-05$ & 7.09E-07 & 3.04E-06 & $2.27 \mathrm{E}-10$ & $7.58 \mathrm{E}-06$ & $1.44 \mathrm{E}-09$ & $9.70 \mathrm{E}-09$ & $8.19 \mathrm{E}-11$ & $2.37 \mathrm{E}-12$ & $2.26 \mathrm{E}-10$ \\
\hline $45 \%$ & $2.15 \mathrm{E}-03$ & $2.29 \mathrm{E}-08$ & $1.50 \mathrm{E}-03$ & $8.68 \mathrm{E}-10$ & 4.67E-04 & $2.20 \mathrm{E}-05$ & 7.61E-07 & 3.32E-06 & $2.33 \mathrm{E}-10$ & $7.58 \mathrm{E}-06$ & $1.49 \mathrm{E}-09$ & $1.03 \mathrm{E}-08$ & $8.42 \mathrm{E}-11$ & $2.52 \mathrm{E}-12$ & $2.40 \mathrm{E}-10$ \\
\hline $50 \%$ & $2.29 \mathrm{E}-03$ & $2.37 \mathrm{E}-08$ & $1.66 \mathrm{E}-03$ & $8.84 \mathrm{E}-10$ & $5.01 \mathrm{E}-04$ & $2.27 \mathrm{E}-05$ & $8.16 \mathrm{E}-07$ & $3.58 \mathrm{E}-06$ & $2.38 \mathrm{E}-10$ & $7.58 \mathrm{E}-06$ & $1.54 \mathrm{E}-09$ & $1.09 \mathrm{E}-08$ & $8.65 \mathrm{E}-11$ & $2.67 \mathrm{E}-12$ & $2.57 \mathrm{E}-10$ \\
\hline $55 \%$ & $2.47 \mathrm{E}-03$ & $2.44 \mathrm{E}-08$ & $1.80 \mathrm{E}-03$ & $8.96 \mathrm{E}-10$ & $5.34 \mathrm{E}-04$ & $2.35 \mathrm{E}-05$ & $8.65 \mathrm{E}-07$ & 3.87E-06 & $2.42 \mathrm{E}-10$ & $7.58 \mathrm{E}-06$ & $1.59 \mathrm{E}-09$ & $1.17 \mathrm{E}-08$ & $8.87 \mathrm{E}-11$ & $2.83 \mathrm{E}-12$ & $2.72 \mathrm{E}-10$ \\
\hline $60 \%$ & $2.65 \mathrm{E}-03$ & $2.50 \mathrm{E}-08$ & $1.99 \mathrm{E}-03$ & $9.05 \mathrm{E}-10$ & $5.77 \mathrm{E}-04$ & $2.42 \mathrm{E}-05$ & $9.35 \mathrm{E}-07$ & 4.14E-06 & $2.45 \mathrm{E}-10$ & $7.58 \mathrm{E}-06$ & $1.62 \mathrm{E}-09$ & $1.25 \mathrm{E}-08$ & $9.13 \mathrm{E}-11$ & $3.01 \mathrm{E}-12$ & $2.86 \mathrm{E}-10$ \\
\hline $65 \%$ & $2.89 \mathrm{E}-03$ & $2.57 \mathrm{E}-08$ & $2.21 \mathrm{E}-03$ & $9.11 \mathrm{E}-10$ & $6.26 \mathrm{E}-04$ & $2.51 \mathrm{E}-05$ & $1.01 \mathrm{E}-06$ & 4.40E-06 & $2.47 \mathrm{E}-10$ & $7.58 \mathrm{E}-06$ & $1.66 \mathrm{E}-09$ & $1.33 \mathrm{E}-08$ & $9.40 \mathrm{E}-11$ & $3.20 \mathrm{E}-12$ & $3.00 \mathrm{E}-10$ \\
\hline $70 \%$ & $3.17 \mathrm{E}-03$ & 2.64E-08 & $2.46 \mathrm{E}-03$ & $9.16 \mathrm{E}-10$ & 6.76E-04 & $2.60 \mathrm{E}-05$ & $1.08 \mathrm{E}-06$ & 4.74E-06 & 2.49E-10 & $7.58 \mathrm{E}-06$ & $1.70 \mathrm{E}-09$ & $1.43 \mathrm{E}-08$ & $9.68 \mathrm{E}-11$ & 3.42E-12 & $3.18 \mathrm{E}-10$ \\
\hline $75 \%$ & 3.49E-03 & $2.73 \mathrm{E}-08$ & $2.80 \mathrm{E}-03$ & $9.20 \mathrm{E}-10$ & $7.30 \mathrm{E}-04$ & $2.69 \mathrm{E}-05$ & $1.17 \mathrm{E}-06$ & $5.07 \mathrm{E}-06$ & $2.50 \mathrm{E}-10$ & $7.58 \mathrm{E}-06$ & $1.74 \mathrm{E}-09$ & $1.55 \mathrm{E}-08$ & $1.00 \mathrm{E}-10$ & 3.66E-12 & $3.37 \mathrm{E}-10$ \\
\hline $80 \%$ & $3.91 \mathrm{E}-03$ & $2.83 \mathrm{E}-08$ & $3.22 \mathrm{E}-03$ & $9.25 \mathrm{E}-10$ & 7.94E-04 & $2.80 \mathrm{E}-05$ & $1.27 \mathrm{E}-06$ & $5.41 \mathrm{E}-06$ & $2.51 \mathrm{E}-10$ & $7.58 \mathrm{E}-06$ & $1.79 \mathrm{E}-09$ & $1.69 \mathrm{E}-08$ & $1.04 \mathrm{E}-10$ & 3.97E-12 & $3.57 \mathrm{E}-10$ \\
\hline $85 \%$ & 4.62E- 03 & 2.93E-08 & 3.92E-03 & $9.36 \mathrm{E}-10$ & 8.76E-04 & $2.93 \mathrm{E}-05$ & $1.40 \mathrm{E}-06$ & $5.88 \mathrm{E}-06$ & $2.52 \mathrm{E}-10$ & 7.58E-06 & $1.84 \mathrm{E}-09$ & $1.87 \mathrm{E}-08$ & $1.08 \mathrm{E}-10$ & $4.35 \mathrm{E}-12$ & $3.84 \mathrm{E}-10$ \\
\hline $90 \%$ & $5.69 \mathrm{E}-03$ & $3.08 \mathrm{E}-08$ & $5.02 \mathrm{E}-03$ & $9.61 \mathrm{E}-10$ & $9.87 \mathrm{E}-04$ & $3.09 \mathrm{E}-05$ & $1.58 \mathrm{E}-06$ & $6.45 \mathrm{E}-06$ & $2.53 \mathrm{E}-10$ & $7.58 \mathrm{E}-06$ & 1.90E-09 & $2.09 \mathrm{E}-08$ & $1.14 \mathrm{E}-10$ & $4.89 \mathrm{E}-12$ & $4.15 \mathrm{E}-10$ \\
\hline $95 \%$ & $8.18 \mathrm{E}-03$ & $3.31 \mathrm{E}-08$ & $7.38 \mathrm{E}-03$ & $1.01 \mathrm{E}-09$ & $1.18 \mathrm{E}-03$ & $3.34 \mathrm{E}-05$ & $1.92 \mathrm{E}-06$ & 7.34E-06 & $2.54 \mathrm{E}-10$ & $7.58 \mathrm{E}-06$ & $1.99 \mathrm{E}-09$ & $2.52 \mathrm{E}-08$ & $1.24 \mathrm{E}-10$ & $5.80 \mathrm{E}-12$ & $4.59 \mathrm{E}-10$ \\
\hline Mean & $3.13 \mathrm{E}-03$ & 2.36E-08 & $2.53 \mathrm{E}-03$ & $7.69 \mathrm{E}-10$ & $5.72 \mathrm{E}-04$ & $2.23 \mathrm{E}-05$ & $9.19 \mathrm{E}-07$ & $3.72 \mathrm{E}-06$ & $2.12 \mathrm{E}-10$ & $7.58 \mathrm{E}-06$ & $1.40 \mathrm{E}-09$ & $1.24 \mathrm{E}-08$ & $8.92 \mathrm{E}-11$ & $2.98 \mathrm{E}-12$ & $2.60 \mathrm{E}-10$ \\
\hline Min & $3.37 \mathrm{E}-04$ & 2.72E-09 & $2.13 \mathrm{E}-04$ & 4.46E-12 & $6.07 \mathrm{E}-05$ & 4.24E-06 & $8.48 \mathrm{E}-08$ & $3.08 \mathrm{E}-08$ & $1.10 \mathrm{E}-10$ & 7.51E-06 & $6.34 \mathrm{E}-12$ & $2.05 \mathrm{E}-09$ & 4.61E-11 & $6.27 \mathrm{E}-13$ & $6.05 \mathrm{E}-12$ \\
\hline Max & 4.32E-02 & 6.72E-08 & $4.25 \mathrm{E}-02$ & $1.25 \mathrm{E}-09$ & $3.09 \mathrm{E}-03$ & 4.83E-05 & $3.72 \mathrm{E}-06$ & $1.21 \mathrm{E}-05$ & $2.56 \mathrm{E}-10$ & $7.58 \mathrm{E}-06$ & $2.37 \mathrm{E}-09$ & $5.38 \mathrm{E}-08$ & $3.31 \mathrm{E}-10$ & $1.24 \mathrm{E}-11$ & $8.37 \mathrm{E}-10$ \\
\hline
\end{tabular}




\section{TABLE C.14 Peak DSR Percentiles (mrem/yr per pCi/g) for Different Exposure Pathways for Pu-239 in Offsite Resident Scenario}

\begin{tabular}{|c|c|c|c|c|c|c|c|c|c|c|c|c|c|}
\hline Percentile & Total & External-WD & Fish & Plant-WD & Meat-WD & Milk-WD & Soil-WD & External-WI & Inhalation & Plant-WI & Meat-WI & Milk-WI & Soil-WI \\
\hline $5 \%$ & $1.04 \mathrm{E}-03$ & $1.50 \mathrm{E}-07$ & $6.68 \mathrm{E}-04$ & $2.32 \mathrm{E}-04$ & $1.03 \mathrm{E}-05$ & $3.22 \mathrm{E}-07$ & $7.83 \mathrm{E}-06$ & $6.93 \mathrm{E}-09$ & $8.30 \mathrm{E}-06$ & $9.77 \mathrm{E}-09$ & $1.49 \mathrm{E}-10$ & $2.60 \mathrm{E}-12$ & $7.67 \mathrm{E}-10$ \\
\hline $10 \%$ & $1.39 \mathrm{E}-03$ & $2.33 \mathrm{E}-07$ & $8.83 \mathrm{E}-04$ & $3.29 \mathrm{E}-04$ & $1.44 \mathrm{E}-05$ & $4.31 \mathrm{E}-07$ & $1.30 \mathrm{E}-05$ & $6.93 \mathrm{E}-09$ & $8.30 \mathrm{E}-06$ & $1.18 \mathrm{E}-08$ & $1.89 \mathrm{E}-10$ & $3.26 \mathrm{E}-12$ & $1.16 \mathrm{E}-09$ \\
\hline $15 \%$ & $1.64 \mathrm{E}-03$ & $2.97 \mathrm{E}-07$ & $9.99 \mathrm{E}-04$ & $3.93 \mathrm{E}-04$ & $1.80 \mathrm{E}-05$ & $5.08 \mathrm{E}-07$ & $1.83 \mathrm{E}-05$ & $6.93 \mathrm{E}-09$ & $8.30 \mathrm{E}-06$ & $1.35 \mathrm{E}-08$ & $2.18 \mathrm{E}-10$ & $3.77 \mathrm{E}-12$ & $1.53 \mathrm{E}-09$ \\
\hline $20 \%$ & $1.82 \mathrm{E}-03$ & $3.55 \mathrm{E}-07$ & $1.10 \mathrm{E}-03$ & $4.48 \mathrm{E}-04$ & $2.09 \mathrm{E}-05$ & $5.77 \mathrm{E}-07$ & $2.26 \mathrm{E}-05$ & $6.93 \mathrm{E}-09$ & $8.30 \mathrm{E}-06$ & $1.50 \mathrm{E}-08$ & $2.39 \mathrm{E}-10$ & $4.22 \mathrm{E}-12$ & $1.84 \mathrm{E}-09$ \\
\hline $25 \%$ & $1.97 \mathrm{E}-03$ & $4.16 \mathrm{E}-07$ & $1.20 \mathrm{E}-03$ & $5.02 \mathrm{E}-04$ & $2.26 \mathrm{E}-05$ & $6.45 \mathrm{E}-07$ & $2.69 \mathrm{E}-05$ & $6.93 \mathrm{E}-09$ & $8.30 \mathrm{E}-06$ & $1.65 \mathrm{E}-08$ & $2.58 \mathrm{E}-10$ & $4.65 \mathrm{E}-12$ & $2.19 \mathrm{E}-09$ \\
\hline $30 \%$ & $2.13 \mathrm{E}-03$ & 4.71E-07 & $1.30 \mathrm{E}-03$ & $5.49 \mathrm{E}-04$ & $2.42 \mathrm{E}-05$ & $7.14 \mathrm{E}-07$ & $3.18 \mathrm{E}-05$ & 6.93E-09 & $8.30 \mathrm{E}-06$ & $1.80 \mathrm{E}-08$ & $2.75 \mathrm{E}-10$ & $5.04 \mathrm{E}-12$ & $2.54 \mathrm{E}-09$ \\
\hline $35 \%$ & $2.29 \mathrm{E}-03$ & 5.31E-07 & $1.43 \mathrm{E}-03$ & $5.91 \mathrm{E}-04$ & $2.56 \mathrm{E}-05$ & $7.71 \mathrm{E}-07$ & 3.61E-05 & $6.93 \mathrm{E}-09$ & $8.30 \mathrm{E}-06$ & $1.95 \mathrm{E}-08$ & $2.90 \mathrm{E}-10$ & $5.45 \mathrm{E}-12$ & $2.85 \mathrm{E}-09$ \\
\hline $40 \%$ & $2.46 \mathrm{E}-03$ & $5.83 \mathrm{E}-07$ & $1.55 \mathrm{E}-03$ & $6.37 \mathrm{E}-04$ & $2.68 \mathrm{E}-05$ & $8.25 \mathrm{E}-07$ & $4.12 \mathrm{E}-05$ & $6.93 \mathrm{E}-09$ & $8.30 \mathrm{E}-06$ & $2.09 \mathrm{E}-08$ & $3.06 \mathrm{E}-10$ & $5.85 \mathrm{E}-12$ & $3.14 \mathrm{E}-09$ \\
\hline $45 \%$ & $2.60 \mathrm{E}-03$ & $6.41 \mathrm{E}-07$ & $1.70 \mathrm{E}-03$ & $6.85 \mathrm{E}-04$ & $2.81 \mathrm{E}-05$ & $8.92 \mathrm{E}-07$ & $4.61 \mathrm{E}-05$ & $6.93 \mathrm{E}-09$ & $8.30 \mathrm{E}-06$ & $2.21 \mathrm{E}-08$ & $3.22 \mathrm{E}-10$ & $6.30 \mathrm{E}-12$ & $3.49 \mathrm{E}-09$ \\
\hline $50 \%$ & $2.77 \mathrm{E}-03$ & $6.93 \mathrm{E}-07$ & $1.87 \mathrm{E}-03$ & $7.38 \mathrm{E}-04$ & $2.92 \mathrm{E}-05$ & $9.60 \mathrm{E}-07$ & $5.11 \mathrm{E}-05$ & $6.93 \mathrm{E}-09$ & $8.30 \mathrm{E}-06$ & $2.37 \mathrm{E}-08$ & $3.39 \mathrm{E}-10$ & $6.80 \mathrm{E}-12$ & $3.80 \mathrm{E}-09$ \\
\hline $55 \%$ & $2.96 \mathrm{E}-03$ & 7.39E-07 & $2.03 \mathrm{E}-03$ & $7.86 \mathrm{E}-04$ & $3.02 \mathrm{E}-05$ & $1.02 \mathrm{E}-06$ & 5.63E-05 & $6.93 \mathrm{E}-09$ & $8.30 \mathrm{E}-06$ & $2.54 \mathrm{E}-08$ & $3.54 \mathrm{E}-10$ & 7.33E-12 & $4.12 \mathrm{E}-09$ \\
\hline $60 \%$ & $3.20 \mathrm{E}-03$ & 7.77E-07 & $2.27 \mathrm{E}-03$ & $8.44 \mathrm{E}-04$ & $3.14 \mathrm{E}-05$ & $1.09 \mathrm{E}-06$ & $6.13 \mathrm{E}-05$ & $6.93 \mathrm{E}-09$ & $8.30 \mathrm{E}-06$ & $2.76 \mathrm{E}-08$ & $3.71 \mathrm{E}-10$ & $7.93 \mathrm{E}-12$ & $4.43 \mathrm{E}-09$ \\
\hline $65 \%$ & $3.44 \mathrm{E}-03$ & 8.17E-07 & $2.51 \mathrm{E}-03$ & $9.06 \mathrm{E}-04$ & $3.26 \mathrm{E}-05$ & $1.17 \mathrm{E}-06$ & $6.74 \mathrm{E}-05$ & $6.93 \mathrm{E}-09$ & $8.30 \mathrm{E}-06$ & $2.98 \mathrm{E}-08$ & $3.89 \mathrm{E}-10$ & $8.53 \mathrm{E}-12$ & $4.76 \mathrm{E}-09$ \\
\hline $70 \%$ & $3.77 \mathrm{E}-03$ & 8.54E-07 & $2.82 \mathrm{E}-03$ & $9.71 \mathrm{E}-04$ & $3.40 \mathrm{E}-05$ & $1.26 \mathrm{E}-06$ & $7.31 \mathrm{E}-05$ & $6.93 \mathrm{E}-09$ & $8.30 \mathrm{E}-06$ & $3.22 \mathrm{E}-08$ & $4.09 \mathrm{E}-10$ & $9.21 \mathrm{E}-12$ & $5.12 \mathrm{E}-09$ \\
\hline $75 \%$ & $4.15 \mathrm{E}-03$ & $8.89 \mathrm{E}-07$ & $3.24 \mathrm{E}-03$ & $1.05 \mathrm{E}-03$ & $3.55 \mathrm{E}-05$ & $1.35 \mathrm{E}-06$ & 7.94E-05 & $6.93 \mathrm{E}-09$ & $8.30 \mathrm{E}-06$ & $3.50 \mathrm{E}-08$ & $4.33 \mathrm{E}-10$ & $1.01 \mathrm{E}-11$ & 5.53E-09 \\
\hline $80 \%$ & $4.70 \mathrm{E}-03$ & $9.24 \mathrm{E}-07$ & $3.80 \mathrm{E}-03$ & $1.14 \mathrm{E}-03$ & $3.71 \mathrm{E}-05$ & $1.47 \mathrm{E}-06$ & $8.66 \mathrm{E}-05$ & $6.93 \mathrm{E}-09$ & $8.30 \mathrm{E}-06$ & $3.86 \mathrm{E}-08$ & $4.63 \mathrm{E}-10$ & $1.11 \mathrm{E}-11$ & $5.96 \mathrm{E}-09$ \\
\hline $85 \%$ & $5.39 \mathrm{E}-03$ & $9.59 \mathrm{E}-07$ & $4.51 \mathrm{E}-03$ & $1.25 \mathrm{E}-03$ & $3.91 \mathrm{E}-05$ & $1.63 \mathrm{E}-06$ & $9.55 \mathrm{E}-05$ & $6.93 \mathrm{E}-09$ & $8.30 \mathrm{E}-06$ & 4.34E-08 & $4.95 \mathrm{E}-10$ & $1.21 \mathrm{E}-11$ & $6.44 \mathrm{E}-09$ \\
\hline $90 \%$ & $6.51 \mathrm{E}-03$ & $1.00 \mathrm{E}-06$ & $5.64 \mathrm{E}-03$ & $1.42 \mathrm{E}-03$ & 4.17E-05 & $1.86 \mathrm{E}-06$ & $1.07 \mathrm{E}-04$ & $6.93 \mathrm{E}-09$ & $8.30 \mathrm{E}-06$ & $5.15 \mathrm{E}-08$ & $5.34 \mathrm{E}-10$ & $1.39 \mathrm{E}-11$ & 7.07E-09 \\
\hline $95 \%$ & $9.05 \mathrm{E}-03$ & $1.06 \mathrm{E}-06$ & $8.27 \mathrm{E}-03$ & $1.75 \mathrm{E}-03$ & $4.55 \mathrm{E}-05$ & $2.21 \mathrm{E}-06$ & $1.23 \mathrm{E}-04$ & $6.93 \mathrm{E}-09$ & $8.30 \mathrm{E}-06$ & $6.64 \mathrm{E}-08$ & $6.03 \mathrm{E}-10$ & $1.65 \mathrm{E}-11$ & $8.01 \mathrm{E}-09$ \\
\hline Mean & 3.67E-03 & $6.50 \mathrm{E}-07$ & $2.87 \mathrm{E}-03$ & $8.28 \mathrm{E}-04$ & $2.89 \mathrm{E}-05$ & $1.07 \mathrm{E}-06$ & $5.61 \mathrm{E}-05$ & $6.93 \mathrm{E}-09$ & $8.30 \mathrm{E}-06$ & $2.91 \mathrm{E}-08$ & $3.53 \mathrm{E}-10$ & $7.88 \mathrm{E}-12$ & $3.99 \mathrm{E}-09$ \\
\hline Min & $3.32 \mathrm{E}-04$ & $9.91 \mathrm{E}-09$ & $1.95 \mathrm{E}-04$ & 5.53E-05 & $4.34 \mathrm{E}-06$ & $1.00 \mathrm{E}-07$ & $3.31 \mathrm{E}-07$ & $6.84 \mathrm{E}-09$ & 8.19E-06 & $3.50 \mathrm{E}-09$ & $5.98 \mathrm{E}-11$ & $9.87 \mathrm{E}-13$ & $8.48 \mathrm{E}-11$ \\
\hline Max & $7.18 \mathrm{E}-02$ & $1.30 \mathrm{E}-06$ & 7.04E-02 & $3.56 \mathrm{E}-03$ & $6.86 \mathrm{E}-05$ & $4.76 \mathrm{E}-06$ & $1.94 \mathrm{E}-04$ & $6.93 \mathrm{E}-09$ & $8.30 \mathrm{E}-06$ & $2.35 \mathrm{E}-07$ & $1.00 \mathrm{E}-09$ & $3.56 \mathrm{E}-11$ & $1.25 \mathrm{E}-08$ \\
\hline
\end{tabular}




\section{TABLE C.15 Peak DSR Percentiles (mrem/yr per pCi/g) for Different Exposure Pathways for Sr-90 in Offsite Resident Scenario}

\begin{tabular}{|c|c|c|c|c|c|c|c|c|c|c|c|c|c|}
\hline Percentile & Total & External-WD & Fish & Plant-WD & Meat-WD & Milk-WD & Soil-WD & External-WI & Inhalation & Plant-WI & Meat-WI & Milk-WI & Soil-WI \\
\hline $5 \%$ & $8.35 \mathrm{E}-04$ & $9.80 \mathrm{E}-07$ & $1.34 \mathrm{E}-04$ & 7.27E-05 & $2.05 \mathrm{E}-04$ & $1.20 \mathrm{E}-04$ & $3.24 \mathrm{E}-08$ & $2.09 \mathrm{E}-06$ & $1.08 \mathrm{E}-08$ & $2.46 \mathrm{E}-09$ & $2.34 \mathrm{E}-09$ & $7.32 \mathrm{E}-10$ & $1.83 \mathrm{E}-12$ \\
\hline $10 \%$ & $9.28 \mathrm{E}-04$ & $1.27 \mathrm{E}-06$ & $1.59 \mathrm{E}-04$ & $8.92 \mathrm{E}-05$ & $2.48 \mathrm{E}-04$ & $1.43 \mathrm{E}-04$ & $4.71 \mathrm{E}-08$ & $2.11 \mathrm{E}-06$ & $1.09 \mathrm{E}-08$ & $3.20 \mathrm{E}-09$ & $3.13 \mathrm{E}-09$ & $9.52 \mathrm{E}-10$ & $2.71 \mathrm{E}-12$ \\
\hline $15 \%$ & $1.01 \mathrm{E}-03$ & $1.50 \mathrm{E}-06$ & $1.84 \mathrm{E}-04$ & $1.02 \mathrm{E}-04$ & $2.80 \mathrm{E}-04$ & $1.62 \mathrm{E}-04$ & $5.90 \mathrm{E}-08$ & $2.12 \mathrm{E}-06$ & $1.10 \mathrm{E}-08$ & $3.87 \mathrm{E}-09$ & $3.97 \mathrm{E}-09$ & $1.15 \mathrm{E}-09$ & $3.36 \mathrm{E}-12$ \\
\hline $20 \%$ & $1.07 \mathrm{E}-03$ & $1.71 \mathrm{E}-06$ & $2.06 \mathrm{E}-04$ & $1.14 \mathrm{E}-04$ & $3.10 \mathrm{E}-04$ & $1.78 \mathrm{E}-04$ & 7.00E-08 & $2.12 \mathrm{E}-06$ & $1.10 \mathrm{E}-08$ & 4.46E-09 & 4.77E-09 & $1.37 \mathrm{E}-09$ & $4.02 \mathrm{E}-12$ \\
\hline $25 \%$ & $1.14 \mathrm{E}-03$ & $1.91 \mathrm{E}-06$ & $2.33 \mathrm{E}-04$ & $1.26 \mathrm{E}-04$ & $3.38 \mathrm{E}-04$ & $1.93 \mathrm{E}-04$ & 8.01E-08 & $2.12 \mathrm{E}-06$ & $1.10 \mathrm{E}-08$ & $5.15 \mathrm{E}-09$ & $5.58 \mathrm{E}-09$ & $1.59 \mathrm{E}-09$ & 4.64E-12 \\
\hline $30 \%$ & $1.22 \mathrm{E}-03$ & $2.08 \mathrm{E}-06$ & $2.59 \mathrm{E}-04$ & $1.39 \mathrm{E}-04$ & $3.65 \mathrm{E}-04$ & $2.07 \mathrm{E}-04$ & $9.03 \mathrm{E}-08$ & $2.13 \mathrm{E}-06$ & $1.10 \mathrm{E}-08$ & $5.87 \mathrm{E}-09$ & $6.35 \mathrm{E}-09$ & $1.85 \mathrm{E}-09$ & $5.21 \mathrm{E}-12$ \\
\hline $35 \%$ & $1.28 \mathrm{E}-03$ & $2.23 \mathrm{E}-06$ & $2.86 \mathrm{E}-04$ & $1.52 \mathrm{E}-04$ & $3.92 \mathrm{E}-04$ & $2.22 \mathrm{E}-04$ & $1.01 \mathrm{E}-07$ & $2.13 \mathrm{E}-06$ & $1.10 \mathrm{E}-08$ & $6.71 \mathrm{E}-09$ & $7.26 \mathrm{E}-09$ & $2.08 \mathrm{E}-09$ & $5.84 \mathrm{E}-12$ \\
\hline $40 \%$ & $1.36 \mathrm{E}-03$ & $2.38 \mathrm{E}-06$ & $3.18 \mathrm{E}-04$ & $1.69 \mathrm{E}-04$ & $4.23 \mathrm{E}-04$ & 2.37E-04 & $1.10 \mathrm{E}-07$ & $2.13 \mathrm{E}-06$ & $1.10 \mathrm{E}-08$ & $7.58 \mathrm{E}-09$ & $8.23 \mathrm{E}-09$ & $2.38 \mathrm{E}-09$ & $6.42 \mathrm{E}-12$ \\
\hline $45 \%$ & $1.43 \mathrm{E}-03$ & $2.51 \mathrm{E}-06$ & $3.54 \mathrm{E}-04$ & $1.86 \mathrm{E}-04$ & 4.53E-04 & $2.52 \mathrm{E}-04$ & $1.20 \mathrm{E}-07$ & $2.13 \mathrm{E}-06$ & $1.10 \mathrm{E}-08$ & $8.72 \mathrm{E}-09$ & $9.24 \mathrm{E}-09$ & $2.70 \mathrm{E}-09$ & $6.99 \mathrm{E}-12$ \\
\hline $50 \%$ & $1.52 \mathrm{E}-03$ & 2.61E-06 & $3.92 \mathrm{E}-04$ & $2.02 \mathrm{E}-04$ & 4.87E-04 & 2.69E-04 & $1.30 \mathrm{E}-07$ & $2.13 \mathrm{E}-06$ & $1.10 \mathrm{E}-08$ & $9.82 \mathrm{E}-09$ & $1.05 \mathrm{E}-08$ & $3.04 \mathrm{E}-09$ & $7.62 \mathrm{E}-12$ \\
\hline $55 \%$ & $1.60 \mathrm{E}-03$ & $2.71 \mathrm{E}-06$ & $4.40 \mathrm{E}-04$ & $2.24 \mathrm{E}-04$ & $5.24 \mathrm{E}-04$ & $2.88 \mathrm{E}-04$ & $1.41 \mathrm{E}-07$ & $2.13 \mathrm{E}-06$ & $1.10 \mathrm{E}-08$ & $1.12 \mathrm{E}-08$ & $1.18 \mathrm{E}-08$ & $3.52 \mathrm{E}-09$ & $8.29 \mathrm{E}-12$ \\
\hline $60 \%$ & $1.70 \mathrm{E}-03$ & $2.81 \mathrm{E}-06$ & 4.92E-04 & $2.48 \mathrm{E}-04$ & $5.68 \mathrm{E}-04$ & $3.06 \mathrm{E}-04$ & $1.52 \mathrm{E}-07$ & $2.13 \mathrm{E}-06$ & $1.10 \mathrm{E}-08$ & $1.28 \mathrm{E}-08$ & $1.35 \mathrm{E}-08$ & 3.99E-09 & $8.90 \mathrm{E}-12$ \\
\hline $65 \%$ & $1.82 \mathrm{E}-03$ & $2.90 \mathrm{E}-06$ & $5.56 \mathrm{E}-04$ & $2.76 \mathrm{E}-04$ & $6.23 \mathrm{E}-04$ & $3.30 \mathrm{E}-04$ & $1.63 \mathrm{E}-07$ & $2.13 \mathrm{E}-06$ & $1.10 \mathrm{E}-08$ & $1.44 \mathrm{E}-08$ & $1.54 \mathrm{E}-08$ & 4.53E-09 & $9.57 \mathrm{E}-12$ \\
\hline $70 \%$ & $1.95 \mathrm{E}-03$ & $3.00 \mathrm{E}-06$ & $6.34 \mathrm{E}-04$ & $3.12 \mathrm{E}-04$ & $6.80 \mathrm{E}-04$ & $3.55 \mathrm{E}-04$ & $1.74 \mathrm{E}-07$ & $2.13 \mathrm{E}-06$ & $1.10 \mathrm{E}-08$ & $1.66 \mathrm{E}-08$ & $1.78 \mathrm{E}-08$ & $5.27 \mathrm{E}-09$ & $1.02 \mathrm{E}-11$ \\
\hline $75 \%$ & $2.13 \mathrm{E}-03$ & $3.11 \mathrm{E}-06$ & 7.31E-04 & $3.57 \mathrm{E}-04$ & 7.65E-04 & $3.84 \mathrm{E}-04$ & $1.87 \mathrm{E}-07$ & $2.13 \mathrm{E}-06$ & $1.10 \mathrm{E}-08$ & $1.96 \mathrm{E}-08$ & $2.08 \mathrm{E}-08$ & $6.18 \mathrm{E}-09$ & $1.10 \mathrm{E}-11$ \\
\hline $80 \%$ & $2.33 \mathrm{E}-03$ & $3.21 \mathrm{E}-06$ & 8.67E-04 & 4.30E-04 & $8.66 \mathrm{E}-04$ & $4.20 \mathrm{E}-04$ & $2.02 \mathrm{E}-07$ & $2.13 \mathrm{E}-06$ & $1.10 \mathrm{E}-08$ & $2.37 \mathrm{E}-08$ & $2.44 \mathrm{E}-08$ & 7.40E-09 & $1.19 \mathrm{E}-11$ \\
\hline $85 \%$ & $2.62 \mathrm{E}-03$ & $3.34 \mathrm{E}-06$ & $1.06 \mathrm{E}-03$ & $5.19 \mathrm{E}-04$ & $1.02 \mathrm{E}-03$ & 4.67E-04 & $2.21 \mathrm{E}-07$ & $2.13 \mathrm{E}-06$ & $1.10 \mathrm{E}-08$ & $2.95 \mathrm{E}-08$ & $3.08 \mathrm{E}-08$ & $9.06 \mathrm{E}-09$ & $1.30 \mathrm{E}-11$ \\
\hline $90 \%$ & $3.09 \mathrm{E}-03$ & $3.49 \mathrm{E}-06$ & $1.35 \mathrm{E}-03$ & $6.71 \mathrm{E}-04$ & $1.28 \mathrm{E}-03$ & $5.46 \mathrm{E}-04$ & $2.45 \mathrm{E}-07$ & $2.13 \mathrm{E}-06$ & $1.10 \mathrm{E}-08$ & $3.86 \mathrm{E}-08$ & $4.00 \mathrm{E}-08$ & $1.22 \mathrm{E}-08$ & $1.44 \mathrm{E}-11$ \\
\hline $95 \%$ & $3.99 \mathrm{E}-03$ & $3.70 \mathrm{E}-06$ & $1.95 \mathrm{E}-03$ & $1.01 \mathrm{E}-03$ & $1.89 \mathrm{E}-03$ & $6.77 \mathrm{E}-04$ & $2.82 \mathrm{E}-07$ & 2.13E-06 & $1.10 \mathrm{E}-08$ & $5.93 \mathrm{E}-08$ & $6.28 \mathrm{E}-08$ & $1.87 \mathrm{E}-08$ & $1.65 \mathrm{E}-11$ \\
\hline Mean & $1.86 \mathrm{E}-03$ & $2.50 \mathrm{E}-06$ & $6.49 \mathrm{E}-04$ & $3.34 \mathrm{E}-04$ & $7.18 \mathrm{E}-04$ & $3.23 \mathrm{E}-04$ & $1.40 \mathrm{E}-07$ & $2.12 \mathrm{E}-06$ & $1.10 \mathrm{E}-08$ & $1.80 \mathrm{E}-08$ & $1.93 \mathrm{E}-08$ & $5.70 \mathrm{E}-09$ & $8.17 \mathrm{E}-12$ \\
\hline Min & $3.60 \mathrm{E}-04$ & $3.22 \mathrm{E}-07$ & 7.43E-05 & $2.32 \mathrm{E}-05$ & $8.90 \mathrm{E}-05$ & $4.80 \mathrm{E}-05$ & $2.25 \mathrm{E}-09$ & $1.69 \mathrm{E}-06$ & $8.78 \mathrm{E}-09$ & $6.76 \mathrm{E}-10$ & $5.37 \mathrm{E}-10$ & $2.42 \mathrm{E}-10$ & $1.31 \mathrm{E}-13$ \\
\hline Max & $3.53 \mathrm{E}-02$ & $1.19 \mathrm{E}-05$ & $1.21 \mathrm{E}-02$ & $7.99 \mathrm{E}-03$ & $3.15 \mathrm{E}-02$ & $8.94 \mathrm{E}-03$ & $1.01 \mathrm{E}-06$ & $2.13 \mathrm{E}-06$ & $1.10 \mathrm{E}-08$ & 4.79E-07 & $1.08 \mathrm{E}-06$ & $4.14 \mathrm{E}-07$ & $4.31 \mathrm{E}-11$ \\
\hline
\end{tabular}




\section{TABLE C.16 Peak DSR Percentiles (mrem/yr per pCi/g) for Different Exposure Pathways for Tc-99 in Offsite Resident Scenario}

\begin{tabular}{|c|c|c|c|c|c|c|c|c|c|c|c|c|c|}
\hline Percentile & Total & External-WD & Fish & Plant-WD & Meat-WD & Milk-WD & Soil-WD & External-WI & Inhalation & Plant-WI & Meat-WI & Milk-WI & Soil-WI \\
\hline $5 \%$ & $1.03 \mathrm{E}-04$ & $4.68 \mathrm{E}-09$ & $9.77 \mathrm{E}-06$ & $4.88 \mathrm{E}-05$ & $6.78 \mathrm{E}-07$ & $1.78 \mathrm{E}-05$ & $1.65 \mathrm{E}-09$ & $1.59 \mathrm{E}-09$ & $8.28 \mathrm{E}-10$ & $1.12 \mathrm{E}-10$ & $4.95 \mathrm{E}-13$ & $7.63 \mathrm{E}-12$ & $4.35 \mathrm{E}-15$ \\
\hline $10 \%$ & $2.50 \mathrm{E}-04$ & $9.03 \mathrm{E}-09$ & $2.18 \mathrm{E}-05$ & $1.21 \mathrm{E}-04$ & $1.76 \mathrm{E}-06$ & $3.97 \mathrm{E}-05$ & $3.65 \mathrm{E}-09$ & $1.59 \mathrm{E}-09$ & $8.31 \mathrm{E}-10$ & $1.39 \mathrm{E}-10$ & $6.94 \mathrm{E}-13$ & $1.07 \mathrm{E}-11$ & $6.23 \mathrm{E}-15$ \\
\hline $15 \%$ & $3.90 \mathrm{E}-04$ & $1.39 \mathrm{E}-08$ & $3.59 \mathrm{E}-05$ & $1.99 \mathrm{E}-04$ & $2.87 \mathrm{E}-06$ & $6.69 \mathrm{E}-05$ & $5.68 \mathrm{E}-09$ & $1.60 \mathrm{E}-09$ & $8.33 \mathrm{E}-10$ & $1.65 \mathrm{E}-10$ & $8.80 \mathrm{E}-13$ & $1.30 \mathrm{E}-11$ & $7.58 \mathrm{E}-15$ \\
\hline $20 \%$ & $5.63 \mathrm{E}-04$ & $2.02 \mathrm{E}-08$ & $5.29 \mathrm{E}-05$ & $2.85 \mathrm{E}-04$ & $4.16 \mathrm{E}-06$ & $9.71 \mathrm{E}-05$ & $8.34 \mathrm{E}-09$ & $1.60 \mathrm{E}-09$ & $8.35 \mathrm{E}-10$ & $1.89 \mathrm{E}-10$ & $1.04 \mathrm{E}-12$ & $1.53 \mathrm{E}-11$ & $8.68 \mathrm{E}-15$ \\
\hline $25 \%$ & $7.84 \mathrm{E}-04$ & $2.74 \mathrm{E}-08$ & $7.27 \mathrm{E}-05$ & $4.02 \mathrm{E}-04$ & $5.66 \mathrm{E}-06$ & $1.34 \mathrm{E}-04$ & $1.12 \mathrm{E}-08$ & $1.61 \mathrm{E}-09$ & $8.37 \mathrm{E}-10$ & $2.10 \mathrm{E}-10$ & $1.21 \mathrm{E}-12$ & $1.79 \mathrm{E}-11$ & $9.70 \mathrm{E}-15$ \\
\hline $30 \%$ & $1.02 \mathrm{E}-03$ & $3.77 \mathrm{E}-08$ & $9.33 \mathrm{E}-05$ & $5.15 \mathrm{E}-04$ & $7.54 \mathrm{E}-06$ & $1.73 \mathrm{E}-04$ & $1.45 \mathrm{E}-08$ & $1.61 \mathrm{E}-09$ & $8.38 \mathrm{E}-10$ & $2.39 \mathrm{E}-10$ & $1.39 \mathrm{E}-12$ & $2.07 \mathrm{E}-11$ & $1.05 \mathrm{E}-14$ \\
\hline $35 \%$ & $1.29 \mathrm{E}-03$ & 4.93E-08 & $1.20 \mathrm{E}-04$ & $6.41 \mathrm{E}-04$ & $9.16 \mathrm{E}-06$ & $2.18 \mathrm{E}-04$ & $1.84 \mathrm{E}-08$ & $1.61 \mathrm{E}-09$ & $8.40 \mathrm{E}-10$ & $2.65 \mathrm{E}-10$ & $1.59 \mathrm{E}-12$ & $2.36 \mathrm{E}-11$ & $1.15 \mathrm{E}-14$ \\
\hline $40 \%$ & $1.53 \mathrm{E}-03$ & $6.12 \mathrm{E}-08$ & $1.47 \mathrm{E}-04$ & $7.81 \mathrm{E}-04$ & $1.10 \mathrm{E}-05$ & $2.59 \mathrm{E}-04$ & $2.28 \mathrm{E}-08$ & $1.61 \mathrm{E}-09$ & $8.42 \mathrm{E}-10$ & $2.92 \mathrm{E}-10$ & $1.81 \mathrm{E}-12$ & $2.66 \mathrm{E}-11$ & $1.23 \mathrm{E}-14$ \\
\hline $45 \%$ & $1.79 \mathrm{E}-03$ & 7.24E-08 & $1.74 \mathrm{E}-04$ & $8.92 \mathrm{E}-04$ & $1.30 \mathrm{E}-05$ & $3.06 \mathrm{E}-04$ & $2.77 \mathrm{E}-08$ & $1.62 \mathrm{E}-09$ & $8.44 \mathrm{E}-10$ & $3.20 \mathrm{E}-10$ & $2.06 \mathrm{E}-12$ & $3.02 \mathrm{E}-11$ & $1.31 \mathrm{E}-14$ \\
\hline $50 \%$ & $2.03 \mathrm{E}-03$ & $8.20 \mathrm{E}-08$ & $2.07 \mathrm{E}-04$ & $1.02 \mathrm{E}-03$ & $1.53 \mathrm{E}-05$ & $3.58 \mathrm{E}-04$ & $3.21 \mathrm{E}-08$ & $1.62 \mathrm{E}-09$ & $8.45 \mathrm{E}-10$ & $3.51 \mathrm{E}-10$ & $2.28 \mathrm{E}-12$ & $3.43 \mathrm{E}-11$ & $1.38 \mathrm{E}-14$ \\
\hline $55 \%$ & $2.31 \mathrm{E}-03$ & 8.91E-08 & $2.44 \mathrm{E}-04$ & $1.16 \mathrm{E}-03$ & $1.76 \mathrm{E}-05$ & $4.14 \mathrm{E}-04$ & $3.71 \mathrm{E}-08$ & $1.62 \mathrm{E}-09$ & $8.47 \mathrm{E}-10$ & $3.86 \mathrm{E}-10$ & $2.57 \mathrm{E}-12$ & $3.89 \mathrm{E}-11$ & $1.46 \mathrm{E}-14$ \\
\hline $60 \%$ & $2.59 \mathrm{E}-03$ & $9.60 \mathrm{E}-08$ & $2.97 \mathrm{E}-04$ & $1.33 \mathrm{E}-03$ & $2.01 \mathrm{E}-05$ & $4.77 \mathrm{E}-04$ & $4.22 \mathrm{E}-08$ & $1.63 \mathrm{E}-09$ & $8.49 \mathrm{E}-10$ & $4.31 \mathrm{E}-10$ & $2.92 \mathrm{E}-12$ & $4.32 \mathrm{E}-11$ & $1.54 \mathrm{E}-14$ \\
\hline $65 \%$ & $2.86 \mathrm{E}-03$ & $1.03 \mathrm{E}-07$ & $3.59 \mathrm{E}-04$ & $1.51 \mathrm{E}-03$ & $2.32 \mathrm{E}-05$ & $5.43 \mathrm{E}-04$ & $4.78 \mathrm{E}-08$ & $1.63 \mathrm{E}-09$ & $8.51 \mathrm{E}-10$ & $4.80 \mathrm{E}-10$ & $3.36 \mathrm{E}-12$ & $4.92 \mathrm{E}-11$ & $1.63 \mathrm{E}-14$ \\
\hline $70 \%$ & $3.18 \mathrm{E}-03$ & $1.10 \mathrm{E}-07$ & $4.24 \mathrm{E}-04$ & $1.72 \mathrm{E}-03$ & $2.72 \mathrm{E}-05$ & $6.25 \mathrm{E}-04$ & $5.34 \mathrm{E}-08$ & $1.63 \mathrm{E}-09$ & $8.52 \mathrm{E}-10$ & $5.39 \mathrm{E}-10$ & $3.86 \mathrm{E}-12$ & $5.64 \mathrm{E}-11$ & $1.73 \mathrm{E}-14$ \\
\hline $75 \%$ & $3.54 \mathrm{E}-03$ & $1.17 \mathrm{E}-07$ & $5.18 \mathrm{E}-04$ & $1.98 \mathrm{E}-03$ & $3.19 \mathrm{E}-05$ & $7.27 \mathrm{E}-04$ & $5.96 \mathrm{E}-08$ & $1.64 \mathrm{E}-09$ & $8.54 \mathrm{E}-10$ & $6.05 \mathrm{E}-10$ & 4. $47 \mathrm{E}-12$ & $6.46 \mathrm{E}-11$ & $1.82 \mathrm{E}-14$ \\
\hline $80 \%$ & $4.02 \mathrm{E}-03$ & $1.25 \mathrm{E}-07$ & $6.50 \mathrm{E}-04$ & $2.30 \mathrm{E}-03$ & $3.81 \mathrm{E}-05$ & $8.45 \mathrm{E}-04$ & $6.67 \mathrm{E}-08$ & $1.64 \mathrm{E}-09$ & $8.56 \mathrm{E}-10$ & $6.92 \mathrm{E}-10$ & $5.31 \mathrm{E}-12$ & $7.54 \mathrm{E}-11$ & $1.95 \mathrm{E}-14$ \\
\hline $85 \%$ & $4.62 \mathrm{E}-03$ & $1.33 \mathrm{E}-07$ & $8.48 \mathrm{E}-04$ & $2.67 \mathrm{E}-03$ & $4.60 \mathrm{E}-05$ & $1.02 \mathrm{E}-03$ & 7.49E-08 & $1.64 \mathrm{E}-09$ & $8.57 \mathrm{E}-10$ & $8.23 \mathrm{E}-10$ & $6.37 \mathrm{E}-12$ & $9.32 \mathrm{E}-11$ & $2.07 \mathrm{E}-14$ \\
\hline $90 \%$ & $5.50 \mathrm{E}-03$ & $1.46 \mathrm{E}-07$ & $1.19 \mathrm{E}-03$ & $3.35 \mathrm{E}-03$ & $5.97 \mathrm{E}-05$ & $1.24 \mathrm{E}-03$ & 8.47E-08 & $1.65 \mathrm{E}-09$ & $8.59 \mathrm{E}-10$ & $1.03 \mathrm{E}-09$ & $8.02 \mathrm{E}-12$ & $1.19 \mathrm{E}-10$ & $2.22 \mathrm{E}-14$ \\
\hline $95 \%$ & $7.06 \mathrm{E}-03$ & $1.63 \mathrm{E}-07$ & $1.82 \mathrm{E}-03$ & 4.70E-03 & $8.63 \mathrm{E}-05$ & $1.75 \mathrm{E}-03$ & $1.00 \mathrm{E}-07$ & $1.65 \mathrm{E}-09$ & 8.61E-10 & $1.39 \mathrm{E}-09$ & $1.18 \mathrm{E}-11$ & $1.71 \mathrm{E}-10$ & $2.49 \mathrm{E}-14$ \\
\hline Mean & $2.59 \mathrm{E}-03$ & $7.80 \mathrm{E}-08$ & 4.92E-04 & $1.53 \mathrm{E}-03$ & $2.57 \mathrm{E}-05$ & $5.48 \mathrm{E}-04$ & $3.91 \mathrm{E}-08$ & $1.62 \mathrm{E}-09$ & $8.45 \mathrm{E}-10$ & $5.01 \mathrm{E}-10$ & $3.70 \mathrm{E}-12$ & $5.54 \mathrm{E}-11$ & $1.41 \mathrm{E}-14$ \\
\hline Min & $3.93 \mathrm{E}-06$ & $3.55 \mathrm{E}-10$ & $1.76 \mathrm{E}-07$ & $1.74 \mathrm{E}-06$ & $1.17 \mathrm{E}-08$ & $5.68 \mathrm{E}-07$ & $1.99 \mathrm{E}-11$ & $1.56 \mathrm{E}-09$ & $8.12 \mathrm{E}-10$ & $3.54 \mathrm{E}-11$ & $8.99 \mathrm{E}-14$ & $1.49 \mathrm{E}-12$ & $1.35 \mathrm{E}-16$ \\
\hline Max & $4.25 \mathrm{E}-02$ & $2.36 \mathrm{E}-07$ & 4.07E-02 & $2.62 \mathrm{E}-02$ & $6.81 \mathrm{E}-04$ & $1.36 \mathrm{E}-02$ & $1.93 \mathrm{E}-07$ & $1.67 \mathrm{E}-09$ & $8.69 \mathrm{E}-10$ & $5.23 \mathrm{E}-09$ & 7.73E-11 & $2.54 \mathrm{E}-09$ & $3.76 \mathrm{E}-14$ \\
\hline
\end{tabular}


Tables C.17-C.24 show the peak DSR percentiles (mrem/yr per $\mathrm{pCi} / \mathrm{g}$ ) for different exposure pathways for individual radionuclides in the recreational use scenario. RESRAD (onsite) version 6.5 was used in the analysis. For C-14 and Tc-99, water-dependent pathways were dominant, and for others (Am-241, Ba-133, Cs-137, Pu-238, Pu-239, and Sr-90), waterindependent pathways were dominant. For Am-241, Pu-238, and Pu-239, meat ingestion, soil ingestion, and inhalation were dominant exposure pathways. For Ba-133, external exposure was dominant. For C-14 and Tc-99, aquatic food ingestion resulted in most dose. For Cs-137, meat ingestion and external exposure resulted in most dose. For Sr-90, meat ingestion resulted in most dose.

TABLE C.17 Peak DSR Percentiles (mrem/yr per pCi/g) for Different Exposure Pathways for Am-241 in Recreational Use Scenario

\begin{tabular}{|c|c|c|c|c|c|c|c|c|}
\hline Percentile & External & $\begin{array}{c}\text { Inhalation } \\
\text { w/o Rn }\end{array}$ & $\begin{array}{c}\text { Meat } \\
\text { Ingestion } \\
\text { (WI) }\end{array}$ & $\begin{array}{c}\text { Soil } \\
\text { Ingestion }\end{array}$ & $\begin{array}{c}\text { Water } \\
\text { Ingestion }\end{array}$ & $\begin{array}{l}\text { Aquatic } \\
\text { Foods }\end{array}$ & $\begin{array}{c}\text { Meat } \\
\text { Ingestion } \\
\text { (WD) }\end{array}$ & $\begin{array}{c}\text { All } \\
\text { Pathways }\end{array}$ \\
\hline $5 \%$ & $1.00 \mathrm{E}-03$ & $1.18 \mathrm{E}-04$ & $1.78 \mathrm{E}-04$ & $1.27 \mathrm{E}-04$ & $0.00 \mathrm{E}+00$ & $0.00 \mathrm{E}+00$ & $0.00 \mathrm{E}+00$ & $1.81 \mathrm{E}-03$ \\
\hline $10 \%$ & $1.11 \mathrm{E}-03$ & 1.63E-04 & $2.18 \mathrm{E}-04$ & $1.84 \mathrm{E}-04$ & $0.00 \mathrm{E}+00$ & $0.00 \mathrm{E}+00$ & $0.00 \mathrm{E}+00$ & $1.96 \mathrm{E}-03$ \\
\hline $15 \%$ & $1.14 \mathrm{E}-03$ & 1.92E-04 & $2.51 \mathrm{E}-04$ & $2.27 \mathrm{E}-04$ & $0.00 \mathrm{E}+00$ & $0.00 \mathrm{E}+00$ & $0.00 \mathrm{E}+00$ & $2.05 \mathrm{E}-03$ \\
\hline $20 \%$ & $1.16 \mathrm{E}-03$ & $2.12 \mathrm{E}-04$ & $2.78 \mathrm{E}-04$ & $2.64 \mathrm{E}-04$ & $0.00 \mathrm{E}+00$ & $0.00 \mathrm{E}+00$ & $0.00 \mathrm{E}+00$ & $2.11 \mathrm{E}-03$ \\
\hline $25 \%$ & $1.17 \mathrm{E}-03$ & $2.28 \mathrm{E}-04$ & $3.01 \mathrm{E}-04$ & $2.95 \mathrm{E}-04$ & $0.00 \mathrm{E}+00$ & $0.00 \mathrm{E}+00$ & $0.00 \mathrm{E}+00$ & $2.16 \mathrm{E}-03$ \\
\hline $30 \%$ & $1.17 \mathrm{E}-03$ & $2.47 \mathrm{E}-04$ & $3.24 \mathrm{E}-04$ & $3.25 \mathrm{E}-04$ & $0.00 \mathrm{E}+00$ & $0.00 \mathrm{E}+00$ & $0.00 \mathrm{E}+00$ & $2.22 \mathrm{E}-03$ \\
\hline $35 \%$ & $1.17 \mathrm{E}-03$ & 2.63E-04 & $3.49 \mathrm{E}-04$ & $3.54 \mathrm{E}-04$ & $0.00 \mathrm{E}+00$ & $0.00 \mathrm{E}+00$ & $0.00 \mathrm{E}+00$ & $2.26 \mathrm{E}-03$ \\
\hline $40 \%$ & $1.17 \mathrm{E}-03$ & $2.80 \mathrm{E}-04$ & $3.73 \mathrm{E}-04$ & $3.81 \mathrm{E}-04$ & $0.00 \mathrm{E}+00$ & $0.00 \mathrm{E}+00$ & $0.00 \mathrm{E}+00$ & $2.31 \mathrm{E}-03$ \\
\hline $45 \%$ & $1.18 \mathrm{E}-03$ & $2.96 \mathrm{E}-04$ & $3.99 \mathrm{E}-04$ & $4.06 \mathrm{E}-04$ & $0.00 \mathrm{E}+00$ & $0.00 \mathrm{E}+00$ & $0.00 \mathrm{E}+00$ & $2.36 \mathrm{E}-03$ \\
\hline $50 \%$ & $1.18 \mathrm{E}-03$ & $3.13 \mathrm{E}-04$ & $4.31 \mathrm{E}-04$ & $4.29 \mathrm{E}-04$ & $0.00 \mathrm{E}+00$ & $0.00 \mathrm{E}+00$ & $0.00 \mathrm{E}+00$ & $2.40 \mathrm{E}-03$ \\
\hline $55 \%$ & $1.18 \mathrm{E}-03$ & $3.34 \mathrm{E}-04$ & $4.60 \mathrm{E}-04$ & $4.50 \mathrm{E}-04$ & $0.00 \mathrm{E}+00$ & $0.00 \mathrm{E}+00$ & $0.00 \mathrm{E}+00$ & $2.44 \mathrm{E}-03$ \\
\hline $60 \%$ & $1.18 \mathrm{E}-03$ & $3.59 \mathrm{E}-04$ & $4.93 \mathrm{E}-04$ & $4.73 \mathrm{E}-04$ & $0.00 \mathrm{E}+00$ & $0.00 \mathrm{E}+00$ & $0.00 \mathrm{E}+00$ & $2.49 \mathrm{E}-03$ \\
\hline $65 \%$ & $1.18 \mathrm{E}-03$ & $3.86 \mathrm{E}-04$ & $5.27 \mathrm{E}-04$ & 4.99E-04 & $0.00 \mathrm{E}+00$ & $0.00 \mathrm{E}+00$ & $0.00 \mathrm{E}+00$ & $2.54 \mathrm{E}-03$ \\
\hline $70 \%$ & $1.18 \mathrm{E}-03$ & $4.12 \mathrm{E}-04$ & $5.63 \mathrm{E}-04$ & $5.27 \mathrm{E}-04$ & $0.00 \mathrm{E}+00$ & $0.00 \mathrm{E}+00$ & $0.00 \mathrm{E}+00$ & $2.58 \mathrm{E}-03$ \\
\hline $75 \%$ & $1.18 \mathrm{E}-03$ & $4.45 \mathrm{E}-04$ & $6.03 \mathrm{E}-04$ & $5.59 \mathrm{E}-04$ & $0.00 \mathrm{E}+00$ & $0.00 \mathrm{E}+00$ & $0.00 \mathrm{E}+00$ & $2.64 \mathrm{E}-03$ \\
\hline $80 \%$ & $1.18 \mathrm{E}-03$ & $4.88 \mathrm{E}-04$ & $6.47 \mathrm{E}-04$ & $5.92 \mathrm{E}-04$ & $0.00 \mathrm{E}+00$ & $0.00 \mathrm{E}+00$ & $0.00 \mathrm{E}+00$ & $2.71 \mathrm{E}-03$ \\
\hline $85 \%$ & $1.18 \mathrm{E}-03$ & $5.47 \mathrm{E}-04$ & $6.98 \mathrm{E}-04$ & $6.27 \mathrm{E}-04$ & $5.86 \mathrm{E}-11$ & $1.68 \mathrm{E}-09$ & $1.23 \mathrm{E}-12$ & $2.79 \mathrm{E}-03$ \\
\hline $90 \%$ & $1.18 \mathrm{E}-03$ & $6.13 \mathrm{E}-04$ & $7.66 \mathrm{E}-04$ & $6.72 \mathrm{E}-04$ & 3.34E-09 & $9.40 \mathrm{E}-08$ & $6.80 \mathrm{E}-11$ & $2.89 \mathrm{E}-03$ \\
\hline $95 \%$ & $1.18 \mathrm{E}-03$ & 7.26E-04 & $8.80 \mathrm{E}-04$ & 7.33E-04 & $4.30 \mathrm{E}-08$ & $1.23 \mathrm{E}-06$ & $9.07 \mathrm{E}-10$ & $3.05 \mathrm{E}-03$ \\
\hline Mean & $1.14 \mathrm{E}-03$ & $3.59 \mathrm{E}-04$ & $4.67 \mathrm{E}-04$ & $4.28 \mathrm{E}-04$ & $6.01 \mathrm{E}-09$ & $2.76 \mathrm{E}-07$ & $1.81 \mathrm{E}-10$ & $2.40 \mathrm{E}-03$ \\
\hline Min & $2.58 \mathrm{E}-08$ & $2.78 \mathrm{E}-10$ & $5.15 \mathrm{E}-09$ & $5.46 \mathrm{E}-10$ & $0.00 \mathrm{E}+00$ & $0.00 \mathrm{E}+00$ & $0.00 \mathrm{E}+00$ & $5.24 \mathrm{E}-08$ \\
\hline Max & $1.18 \mathrm{E}-03$ & $2.55 \mathrm{E}-03$ & $1.46 \mathrm{E}-03$ & $8.80 \mathrm{E}-04$ & $2.24 \mathrm{E}-07$ & $4.41 \mathrm{E}-05$ & $1.58 \mathrm{E}-08$ & $4.99 \mathrm{E}-03$ \\
\hline
\end{tabular}


TABLE C.18 Peak DSR Percentiles (mrem/yr per pCi/g) for Different Exposure Pathways for Ba-133 in Recreational Use Scenario

\begin{tabular}{|c|c|c|c|c|c|c|c|c|}
\hline Percentile & External & $\begin{array}{c}\text { Inhalation } \\
\text { w/o Rn }\end{array}$ & $\begin{array}{c}\text { Meat } \\
\text { Ingestion } \\
(\mathrm{WI})\end{array}$ & $\begin{array}{c}\text { Soil } \\
\text { Ingestion }\end{array}$ & $\begin{array}{c}\text { Water } \\
\text { Ingestion }\end{array}$ & $\begin{array}{c}\text { Aquatic } \\
\text { Foods }\end{array}$ & $\begin{array}{c}\text { Meat } \\
\text { Ingestion } \\
\text { (WD) }\end{array}$ & $\begin{array}{c}\text { All } \\
\text { Pathways }\end{array}$ \\
\hline $5 \%$ & $2.04 \mathrm{E}-07$ & $1.38 \mathrm{E}-13$ & $5.50 \mathrm{E}-11$ & $1.10 \mathrm{E}-11$ & $0.00 \mathrm{E}+00$ & $0.00 \mathrm{E}+00$ & $0.00 \mathrm{E}+00$ & $2.31 \mathrm{E}-07$ \\
\hline $10 \%$ & $1.27 \mathrm{E}-05$ & $6.12 \mathrm{E}-12$ & 2.11E-09 & $4.93 \mathrm{E}-10$ & $0.00 \mathrm{E}+00$ & $0.00 \mathrm{E}+00$ & $0.00 \mathrm{E}+00$ & $1.27 \mathrm{E}-05$ \\
\hline $15 \%$ & 4.04E-05 & $1.74 \mathrm{E}-11$ & 4.67E-09 & $1.35 \mathrm{E}-09$ & $0.00 \mathrm{E}+00$ & $0.00 \mathrm{E}+00$ & $0.00 \mathrm{E}+00$ & 4.04E-05 \\
\hline $20 \%$ & $6.10 \mathrm{E}-05$ & $2.41 \mathrm{E}-11$ & $6.91 \mathrm{E}-09$ & 2.02E-09 & $0.00 \mathrm{E}+00$ & $0.00 \mathrm{E}+00$ & $0.00 \mathrm{E}+00$ & $6.11 \mathrm{E}-05$ \\
\hline $25 \%$ & 7.57E-05 & 3.04E-11 & $9.15 \mathrm{E}-09$ & 2.61E-09 & $0.00 \mathrm{E}+00$ & $0.00 \mathrm{E}+00$ & $0.00 \mathrm{E}+00$ & $7.58 \mathrm{E}-05$ \\
\hline $30 \%$ & 8.46E-05 & $3.52 \mathrm{E}-11$ & $1.12 \mathrm{E}-08$ & $3.12 \mathrm{E}-09$ & $0.00 \mathrm{E}+00$ & $0.00 \mathrm{E}+00$ & $0.00 \mathrm{E}+00$ & $8.46 \mathrm{E}-05$ \\
\hline $35 \%$ & $9.03 \mathrm{E}-05$ & $3.88 \mathrm{E}-11$ & $1.34 \mathrm{E}-08$ & $3.59 \mathrm{E}-09$ & $0.00 \mathrm{E}+00$ & $0.00 \mathrm{E}+00$ & $0.00 \mathrm{E}+00$ & $9.04 \mathrm{E}-05$ \\
\hline $40 \%$ & $9.36 \mathrm{E}-05$ & $4.26 \mathrm{E}-11$ & $1.60 \mathrm{E}-08$ & 4.01E-09 & $0.00 \mathrm{E}+00$ & $0.00 \mathrm{E}+00$ & $0.00 \mathrm{E}+00$ & $9.37 \mathrm{E}-05$ \\
\hline $45 \%$ & $9.61 \mathrm{E}-05$ & $4.62 \mathrm{E}-11$ & $1.89 \mathrm{E}-08$ & 4.40E-09 & $0.00 \mathrm{E}+00$ & $0.00 \mathrm{E}+00$ & $0.00 \mathrm{E}+00$ & $9.62 \mathrm{E}-05$ \\
\hline $50 \%$ & $9.76 \mathrm{E}-05$ & 4.99E-11 & $2.18 \mathrm{E}-08$ & 4.83E-09 & $0.00 \mathrm{E}+00$ & $0.00 \mathrm{E}+00$ & $0.00 \mathrm{E}+00$ & $9.77 \mathrm{E}-05$ \\
\hline $55 \%$ & $9.87 \mathrm{E}-05$ & $5.35 \mathrm{E}-11$ & $2.48 \mathrm{E}-08$ & $5.20 \mathrm{E}-09$ & $0.00 \mathrm{E}+00$ & $0.00 \mathrm{E}+00$ & $0.00 \mathrm{E}+00$ & $9.88 \mathrm{E}-05$ \\
\hline $60 \%$ & $9.95 \mathrm{E}-05$ & $5.70 \mathrm{E}-11$ & $2.88 \mathrm{E}-08$ & $5.58 \mathrm{E}-09$ & $0.00 \mathrm{E}+00$ & $0.00 \mathrm{E}+00$ & $0.00 \mathrm{E}+00$ & $9.95 \mathrm{E}-05$ \\
\hline $65 \%$ & $1.00 \mathrm{E}-04$ & $6.20 \mathrm{E}-11$ & 3.37E-08 & 5.97E-09 & $0.00 \mathrm{E}+00$ & $0.00 \mathrm{E}+00$ & $0.00 \mathrm{E}+00$ & $1.00 \mathrm{E}-04$ \\
\hline $70 \%$ & $1.00 \mathrm{E}-04$ & $6.76 \mathrm{E}-11$ & $3.97 \mathrm{E}-08$ & 6.38E-09 & $0.00 \mathrm{E}+00$ & $0.00 \mathrm{E}+00$ & $0.00 \mathrm{E}+00$ & $1.00 \mathrm{E}-04$ \\
\hline $75 \%$ & $1.01 \mathrm{E}-04$ & $7.35 \mathrm{E}-11$ & 4.71E-08 & 6.79E-09 & $0.00 \mathrm{E}+00$ & $0.00 \mathrm{E}+00$ & $0.00 \mathrm{E}+00$ & $1.01 \mathrm{E}-04$ \\
\hline $80 \%$ & $1.01 \mathrm{E}-04$ & $8.02 \mathrm{E}-11$ & $5.66 \mathrm{E}-08$ & 7.21E-09 & $0.00 \mathrm{E}+00$ & $0.00 \mathrm{E}+00$ & $0.00 \mathrm{E}+00$ & $1.01 \mathrm{E}-04$ \\
\hline $85 \%$ & $1.01 \mathrm{E}-04$ & 8.97E-11 & $7.08 \mathrm{E}-08$ & 7.81E-09 & $0.00 \mathrm{E}+00$ & $0.00 \mathrm{E}+00$ & $0.00 \mathrm{E}+00$ & $1.01 \mathrm{E}-04$ \\
\hline $90 \%$ & $1.01 \mathrm{E}-04$ & $1.04 \mathrm{E}-10$ & $9.39 \mathrm{E}-08$ & 8.47E-09 & $0.00 \mathrm{E}+00$ & $0.00 \mathrm{E}+00$ & $0.00 \mathrm{E}+00$ & $1.01 \mathrm{E}-04$ \\
\hline $95 \%$ & $1.01 \mathrm{E}-04$ & $1.25 \mathrm{E}-10$ & $1.44 \mathrm{E}-07$ & $9.30 \mathrm{E}-09$ & $0.00 \mathrm{E}+00$ & $0.00 \mathrm{E}+00$ & $0.00 \mathrm{E}+00$ & $1.01 \mathrm{E}-04$ \\
\hline Mean & 8.03E-05 & $5.49 \mathrm{E}-11$ & $4.16 \mathrm{E}-08$ & 4.73E-09 & $5.71 \mathrm{E}-11$ & $3.77 \mathrm{E}-10$ & $2.55 \mathrm{E}-13$ & $8.04 \mathrm{E}-05$ \\
\hline Min & $0.00 \mathrm{E}+00$ & $0.00 \mathrm{E}+00$ & $0.00 \mathrm{E}+00$ & $0.00 \mathrm{E}+00$ & $0.00 \mathrm{E}+00$ & $0.00 \mathrm{E}+00$ & $0.00 \mathrm{E}+00$ & $0.00 \mathrm{E}+00$ \\
\hline Max & $1.01 \mathrm{E}-04$ & $4.74 \mathrm{E}-10$ & $2.23 \mathrm{E}-06$ & $1.17 \mathrm{E}-08$ & $7.26 \mathrm{E}-08$ & $4.96 \mathrm{E}-07$ & $3.21 \mathrm{E}-10$ & $1.02 \mathrm{E}-04$ \\
\hline
\end{tabular}


TABLE C.19 Peak DSR Percentiles (mrem/yr per pCi/g) for Different Exposure Pathways for C-14 in Recreational Use Scenario

\begin{tabular}{|c|c|c|c|c|c|c|c|c|}
\hline Percentile & External & $\begin{array}{c}\text { Inhalation } \\
\text { w/o Rn }\end{array}$ & $\begin{array}{c}\text { Meat } \\
\text { Ingestion } \\
(\mathrm{WI})\end{array}$ & $\begin{array}{c}\text { Soil } \\
\text { Ingestion }\end{array}$ & $\begin{array}{c}\text { Water } \\
\text { Ingestion }\end{array}$ & $\begin{array}{c}\text { Aquatic } \\
\text { Foods }\end{array}$ & $\begin{array}{c}\text { Meat } \\
\text { Ingestion } \\
\text { (WD) }\end{array}$ & $\begin{array}{c}\text { All } \\
\text { Pathways }\end{array}$ \\
\hline $5 \%$ & $0.00 \mathrm{E}+00$ & $0.00 \mathrm{E}+00$ & $0.00 \mathrm{E}+00$ & $0.00 \mathrm{E}+00$ & $0.00 \mathrm{E}+00$ & $0.00 \mathrm{E}+00$ & $0.00 \mathrm{E}+00$ & $0.00 \mathrm{E}+00$ \\
\hline $10 \%$ & $0.00 \mathrm{E}+00$ & $0.00 \mathrm{E}+00$ & $0.00 \mathrm{E}+00$ & $0.00 \mathrm{E}+00$ & $0.00 \mathrm{E}+00$ & $0.00 \mathrm{E}+00$ & $0.00 \mathrm{E}+00$ & $0.00 \mathrm{E}+00$ \\
\hline $15 \%$ & $0.00 \mathrm{E}+00$ & $0.00 \mathrm{E}+00$ & $0.00 \mathrm{E}+00$ & $0.00 \mathrm{E}+00$ & $0.00 \mathrm{E}+00$ & $0.00 \mathrm{E}+00$ & $0.00 \mathrm{E}+00$ & $0.00 \mathrm{E}+00$ \\
\hline $20 \%$ & $0.00 \mathrm{E}+00$ & $0.00 \mathrm{E}+00$ & $0.00 \mathrm{E}+00$ & $0.00 \mathrm{E}+00$ & $0.00 \mathrm{E}+00$ & $0.00 \mathrm{E}+00$ & $0.00 \mathrm{E}+00$ & $0.00 \mathrm{E}+00$ \\
\hline $25 \%$ & $0.00 \mathrm{E}+00$ & $0.00 \mathrm{E}+00$ & $0.00 \mathrm{E}+00$ & $0.00 \mathrm{E}+00$ & $0.00 \mathrm{E}+00$ & $0.00 \mathrm{E}+00$ & $0.00 \mathrm{E}+00$ & $0.00 \mathrm{E}+00$ \\
\hline $30 \%$ & $0.00 \mathrm{E}+00$ & $0.00 \mathrm{E}+00$ & $0.00 \mathrm{E}+00$ & $0.00 \mathrm{E}+00$ & $0.00 \mathrm{E}+00$ & $0.00 \mathrm{E}+00$ & $0.00 \mathrm{E}+00$ & $0.00 \mathrm{E}+00$ \\
\hline $35 \%$ & $0.00 \mathrm{E}+00$ & $0.00 \mathrm{E}+00$ & $0.00 \mathrm{E}+00$ & $0.00 \mathrm{E}+00$ & $0.00 \mathrm{E}+00$ & $0.00 \mathrm{E}+00$ & $0.00 \mathrm{E}+00$ & $0.00 \mathrm{E}+00$ \\
\hline $40 \%$ & $0.00 \mathrm{E}+00$ & $0.00 \mathrm{E}+00$ & $0.00 \mathrm{E}+00$ & $0.00 \mathrm{E}+00$ & $0.00 \mathrm{E}+00$ & $0.00 \mathrm{E}+00$ & $0.00 \mathrm{E}+00$ & $0.00 \mathrm{E}+00$ \\
\hline $45 \%$ & $0.00 \mathrm{E}+00$ & $0.00 \mathrm{E}+00$ & $0.00 \mathrm{E}+00$ & $0.00 \mathrm{E}+00$ & $0.00 \mathrm{E}+00$ & $0.00 \mathrm{E}+00$ & $0.00 \mathrm{E}+00$ & $0.00 \mathrm{E}+00$ \\
\hline $50 \%$ & $0.00 \mathrm{E}+00$ & $0.00 \mathrm{E}+00$ & $0.00 \mathrm{E}+00$ & $0.00 \mathrm{E}+00$ & $0.00 \mathrm{E}+00$ & $0.00 \mathrm{E}+00$ & $0.00 \mathrm{E}+00$ & $0.00 \mathrm{E}+00$ \\
\hline $55 \%$ & $0.00 \mathrm{E}+00$ & $0.00 \mathrm{E}+00$ & $0.00 \mathrm{E}+00$ & $0.00 \mathrm{E}+00$ & $0.00 \mathrm{E}+00$ & $0.00 \mathrm{E}+00$ & $0.00 \mathrm{E}+00$ & $0.00 \mathrm{E}+00$ \\
\hline $60 \%$ & $0.00 \mathrm{E}+00$ & $0.00 \mathrm{E}+00$ & $0.00 \mathrm{E}+00$ & $0.00 \mathrm{E}+00$ & $0.00 \mathrm{E}+00$ & $0.00 \mathrm{E}+00$ & $0.00 \mathrm{E}+00$ & $0.00 \mathrm{E}+00$ \\
\hline $65 \%$ & $0.00 \mathrm{E}+00$ & $0.00 \mathrm{E}+00$ & $0.00 \mathrm{E}+00$ & $0.00 \mathrm{E}+00$ & $0.00 \mathrm{E}+00$ & $0.00 \mathrm{E}+00$ & $0.00 \mathrm{E}+00$ & $0.00 \mathrm{E}+00$ \\
\hline $70 \%$ & $0.00 \mathrm{E}+00$ & $0.00 \mathrm{E}+00$ & $0.00 \mathrm{E}+00$ & $0.00 \mathrm{E}+00$ & $1.24 \mathrm{E}-21$ & $1.71 \mathrm{E}-17$ & $9.54 \mathrm{E}-22$ & $1.77 \mathrm{E}-17$ \\
\hline $75 \%$ & $0.00 \mathrm{E}+00$ & $0.00 \mathrm{E}+00$ & $0.00 \mathrm{E}+00$ & $0.00 \mathrm{E}+00$ & $2.58 \mathrm{E}-07$ & $4.23 \mathrm{E}-03$ & $1.88 \mathrm{E}-07$ & $4.24 \mathrm{E}-03$ \\
\hline $80 \%$ & $0.00 \mathrm{E}+00$ & $0.00 \mathrm{E}+00$ & $0.00 \mathrm{E}+00$ & $0.00 \mathrm{E}+00$ & $5.02 \mathrm{E}-07$ & $1.55 \mathrm{E}-02$ & $3.84 \mathrm{E}-07$ & $1.55 \mathrm{E}-02$ \\
\hline $85 \%$ & $0.00 \mathrm{E}+00$ & $0.00 \mathrm{E}+00$ & $0.00 \mathrm{E}+00$ & $0.00 \mathrm{E}+00$ & $9.02 \mathrm{E}-07$ & $5.45 \mathrm{E}-02$ & $6.66 \mathrm{E}-07$ & $5.45 \mathrm{E}-02$ \\
\hline $90 \%$ & $6.11 \mathrm{E}-08$ & $3.03 \mathrm{E}-06$ & $6.00 \mathrm{E}-02$ & $1.62 \mathrm{E}-07$ & $1.48 \mathrm{E}-06$ & $1.17 \mathrm{E}-01$ & $1.17 \mathrm{E}-06$ & $1.17 \mathrm{E}-01$ \\
\hline $95 \%$ & $6.19 \mathrm{E}-08$ & $3.90 \mathrm{E}-06$ & $9.22 \mathrm{E}-02$ & $2.56 \mathrm{E}-07$ & $2.81 \mathrm{E}-06$ & $2.50 \mathrm{E}-01$ & $2.26 \mathrm{E}-06$ & $2.50 \mathrm{E}-01$ \\
\hline Mean & $8.40 \mathrm{E}-09$ & $5.08 \mathrm{E}-07$ & $1.20 \mathrm{E}-02$ & $3.04 \mathrm{E}-08$ & $4.66 \mathrm{E}-07$ & $4.61 \mathrm{E}-02$ & 3.97E-07 & 4.61E-02 \\
\hline Min & $0.00 \mathrm{E}+00$ & $0.00 \mathrm{E}+00$ & $0.00 \mathrm{E}+00$ & $0.00 \mathrm{E}+00$ & $0.00 \mathrm{E}+00$ & $0.00 \mathrm{E}+00$ & $0.00 \mathrm{E}+00$ & $0.00 \mathrm{E}+00$ \\
\hline Max & $6.26 \mathrm{E}-08$ & $9.13 \mathrm{E}-06$ & $3.04 \mathrm{E}-01$ & $4.30 \mathrm{E}-07$ & $1.53 \mathrm{E}-05$ & $1.14 \mathrm{E}+01$ & $2.02 \mathrm{E}-05$ & $1.14 \mathrm{E}+01$ \\
\hline
\end{tabular}


TABLE C.20 Peak DSR Percentiles (mrem/yr per pCi/g) for Different Exposure Pathways for Cs-137 in Recreational Use Scenario

\begin{tabular}{|c|c|c|c|c|c|c|c|c|}
\hline Percentile & External & $\begin{array}{c}\text { Inhalation } \\
\text { w/o Rn }\end{array}$ & $\begin{array}{c}\text { Meat } \\
\text { Ingestion } \\
(\mathrm{WI})\end{array}$ & $\begin{array}{c}\text { Soil } \\
\text { Ingestion }\end{array}$ & $\begin{array}{c}\text { Water } \\
\text { Ingestion }\end{array}$ & $\begin{array}{l}\text { Aquatic } \\
\text { Foods }\end{array}$ & $\begin{array}{c}\text { Meat } \\
\text { Ingestion } \\
\text { (WD) }\end{array}$ & $\begin{array}{c}\text { All } \\
\text { Pathways }\end{array}$ \\
\hline $5 \%$ & $9.79 \mathrm{E}-03$ & 5.80E-09 & $1.90 \mathrm{E}-03$ & $9.80 \mathrm{E}-07$ & $0.00 \mathrm{E}+00$ & $0.00 \mathrm{E}+00$ & $0.00 \mathrm{E}+00$ & $1.27 \mathrm{E}-02$ \\
\hline $10 \%$ & $1.04 \mathrm{E}-02$ & 7.82E-09 & $2.38 \mathrm{E}-03$ & $1.42 \mathrm{E}-06$ & $0.00 \mathrm{E}+00$ & $0.00 \mathrm{E}+00$ & $0.00 \mathrm{E}+00$ & $1.33 \mathrm{E}-02$ \\
\hline $15 \%$ & $1.07 \mathrm{E}-02$ & 8.99E-09 & $2.84 \mathrm{E}-03$ & $1.73 \mathrm{E}-06$ & $0.00 \mathrm{E}+00$ & $0.00 \mathrm{E}+00$ & $0.00 \mathrm{E}+00$ & $1.37 \mathrm{E}-02$ \\
\hline $20 \%$ & $1.09 \mathrm{E}-02$ & 9.87E-09 & $3.23 \mathrm{E}-03$ & $2.01 \mathrm{E}-06$ & $0.00 \mathrm{E}+00$ & $0.00 \mathrm{E}+00$ & $0.00 \mathrm{E}+00$ & $1.42 \mathrm{E}-02$ \\
\hline $25 \%$ & $1.10 \mathrm{E}-02$ & $1.07 \mathrm{E}-08$ & $3.67 \mathrm{E}-03$ & $2.23 \mathrm{E}-06$ & $0.00 \mathrm{E}+00$ & $0.00 \mathrm{E}+00$ & $0.00 \mathrm{E}+00$ & $1.46 \mathrm{E}-02$ \\
\hline $30 \%$ & $1.10 \mathrm{E}-02$ & $1.15 \mathrm{E}-08$ & 4.11E-03 & $2.46 \mathrm{E}-06$ & $0.00 \mathrm{E}+00$ & $0.00 \mathrm{E}+00$ & $0.00 \mathrm{E}+00$ & $1.51 \mathrm{E}-02$ \\
\hline $35 \%$ & $1.11 \mathrm{E}-02$ & $1.23 \mathrm{E}-08$ & $4.51 \mathrm{E}-03$ & $2.65 \mathrm{E}-06$ & $0.00 \mathrm{E}+00$ & $0.00 \mathrm{E}+00$ & $0.00 \mathrm{E}+00$ & $1.55 \mathrm{E}-02$ \\
\hline $40 \%$ & $1.11 \mathrm{E}-02$ & $1.31 \mathrm{E}-08$ & $5.03 \mathrm{E}-03$ & $2.85 \mathrm{E}-06$ & $0.00 \mathrm{E}+00$ & $0.00 \mathrm{E}+00$ & $0.00 \mathrm{E}+00$ & $1.60 \mathrm{E}-02$ \\
\hline $45 \%$ & $1.11 \mathrm{E}-02$ & $1.38 \mathrm{E}-08$ & $5.52 \mathrm{E}-03$ & $3.03 \mathrm{E}-06$ & $0.00 \mathrm{E}+00$ & $0.00 \mathrm{E}+00$ & $0.00 \mathrm{E}+00$ & $1.65 \mathrm{E}-02$ \\
\hline $50 \%$ & $1.12 \mathrm{E}-02$ & $1.46 \mathrm{E}-08$ & 6.09E-03 & $3.21 \mathrm{E}-06$ & $0.00 \mathrm{E}+00$ & $0.00 \mathrm{E}+00$ & $0.00 \mathrm{E}+00$ & $1.71 \mathrm{E}-02$ \\
\hline $55 \%$ & $1.12 \mathrm{E}-02$ & $1.56 \mathrm{E}-08$ & $6.61 \mathrm{E}-03$ & 3.37E-06 & $0.00 \mathrm{E}+00$ & $0.00 \mathrm{E}+00$ & $0.00 \mathrm{E}+00$ & $1.77 \mathrm{E}-02$ \\
\hline $60 \%$ & $1.12 \mathrm{E}-02$ & $1.67 \mathrm{E}-08$ & 7.32E-03 & $3.55 \mathrm{E}-06$ & $0.00 \mathrm{E}+00$ & $0.00 \mathrm{E}+00$ & $0.00 \mathrm{E}+00$ & $1.83 \mathrm{E}-02$ \\
\hline $65 \%$ & $1.12 \mathrm{E}-02$ & $1.79 \mathrm{E}-08$ & $8.15 \mathrm{E}-03$ & $3.75 \mathrm{E}-06$ & $0.00 \mathrm{E}+00$ & $0.00 \mathrm{E}+00$ & $0.00 \mathrm{E}+00$ & $1.92 \mathrm{E}-02$ \\
\hline $70 \%$ & $1.12 \mathrm{E}-02$ & $1.92 \mathrm{E}-08$ & $9.00 \mathrm{E}-03$ & $3.94 \mathrm{E}-06$ & $0.00 \mathrm{E}+00$ & $0.00 \mathrm{E}+00$ & $0.00 \mathrm{E}+00$ & $2.00 \mathrm{E}-02$ \\
\hline $75 \%$ & $1.13 \mathrm{E}-02$ & $2.07 \mathrm{E}-08$ & $1.01 \mathrm{E}-02$ & 4.17E-06 & $0.00 \mathrm{E}+00$ & $0.00 \mathrm{E}+00$ & $0.00 \mathrm{E}+00$ & $2.11 \mathrm{E}-02$ \\
\hline $80 \%$ & $1.13 \mathrm{E}-02$ & $2.26 \mathrm{E}-08$ & $1.16 \mathrm{E}-02$ & $4.40 \mathrm{E}-06$ & $0.00 \mathrm{E}+00$ & $0.00 \mathrm{E}+00$ & $0.00 \mathrm{E}+00$ & $2.25 \mathrm{E}-02$ \\
\hline $85 \%$ & $1.13 \mathrm{E}-02$ & $2.55 \mathrm{E}-08$ & $1.35 \mathrm{E}-02$ & $4.67 \mathrm{E}-06$ & $0.00 \mathrm{E}+00$ & $0.00 \mathrm{E}+00$ & $0.00 \mathrm{E}+00$ & $2.45 \mathrm{E}-02$ \\
\hline $90 \%$ & $1.13 \mathrm{E}-02$ & $2.87 \mathrm{E}-08$ & $1.65 \mathrm{E}-02$ & $5.00 \mathrm{E}-06$ & $0.00 \mathrm{E}+00$ & $0.00 \mathrm{E}+00$ & $0.00 \mathrm{E}+00$ & $2.76 \mathrm{E}-02$ \\
\hline $95 \%$ & $1.13 \mathrm{E}-02$ & $3.35 \mathrm{E}-08$ & $2.38 \mathrm{E}-02$ & $5.43 \mathrm{E}-06$ & $0.00 \mathrm{E}+00$ & $0.00 \mathrm{E}+00$ & $0.00 \mathrm{E}+00$ & $3.49 \mathrm{E}-02$ \\
\hline Mean & $1.09 \mathrm{E}-02$ & $1.68 \mathrm{E}-08$ & $8.62 \mathrm{E}-03$ & $3.20 \mathrm{E}-06$ & $0.00 \mathrm{E}+00$ & $0.00 \mathrm{E}+00$ & $0.00 \mathrm{E}+00$ & $1.95 \mathrm{E}-02$ \\
\hline Min & $1.69 \mathrm{E}-04$ & $3.90 \mathrm{E}-11$ & $4.22 \mathrm{E}-05$ & 4.19E-08 & $0.00 \mathrm{E}+00$ & $0.00 \mathrm{E}+00$ & $0.00 \mathrm{E}+00$ & $2.11 \mathrm{E}-04$ \\
\hline Max & $1.13 \mathrm{E}-02$ & $1.19 \mathrm{E}-07$ & $1.80 \mathrm{E}-01$ & $6.50 \mathrm{E}-06$ & $0.00 \mathrm{E}+00$ & $0.00 \mathrm{E}+00$ & $0.00 \mathrm{E}+00$ & $1.91 \mathrm{E}-01$ \\
\hline
\end{tabular}


TABLE C.21 Peak DSR Percentiles (mrem/yr per pCi/g) for Different Exposure Pathways for Pu-238 in Recreational Use Scenario

\begin{tabular}{|c|c|c|c|c|c|c|c|c|c|}
\hline Percentile & External & $\begin{array}{c}\text { Inhalation } \\
\text { w/o Rn }\end{array}$ & $\begin{array}{l}\text { Radon } \\
\text { (WI) }\end{array}$ & $\begin{array}{c}\text { Meat } \\
\text { Ingestion } \\
(\mathrm{WI})\end{array}$ & $\begin{array}{c}\text { Soil } \\
\text { Ingestion }\end{array}$ & $\begin{array}{c}\text { Water } \\
\text { Ingestion }\end{array}$ & $\begin{array}{l}\text { Aquatic } \\
\text { Foods }\end{array}$ & $\begin{array}{c}\text { Meat } \\
\text { Ingestion } \\
\text { (WD) }\end{array}$ & $\begin{array}{c}\text { All } \\
\text { Pathways }\end{array}$ \\
\hline $5 \%$ & $1.78 \mathrm{E}-06$ & $7.65 \mathrm{E}-05$ & $6.21 \mathrm{E}-14$ & $2.25 \mathrm{E}-04$ & 7.97E-05 & $0.00 \mathrm{E}+00$ & $0.00 \mathrm{E}+00$ & $0.00 \mathrm{E}+00$ & $6.07 \mathrm{E}-04$ \\
\hline $10 \%$ & $1.90 \mathrm{E}-06$ & $1.02 \mathrm{E}-04$ & $7.85 \mathrm{E}-14$ & $2.74 \mathrm{E}-04$ & $1.15 \mathrm{E}-04$ & $0.00 \mathrm{E}+00$ & $0.00 \mathrm{E}+00$ & $0.00 \mathrm{E}+00$ & $6.87 \mathrm{E}-04$ \\
\hline $15 \%$ & $1.94 \mathrm{E}-06$ & $1.18 \mathrm{E}-04$ & $9.13 \mathrm{E}-14$ & $3.07 \mathrm{E}-04$ & $1.43 \mathrm{E}-04$ & $0.00 \mathrm{E}+00$ & $0.00 \mathrm{E}+00$ & $0.00 \mathrm{E}+00$ & 7.47E-04 \\
\hline $20 \%$ & $1.96 \mathrm{E}-06$ & $1.30 \mathrm{E}-04$ & $1.01 \mathrm{E}-13$ & $3.42 \mathrm{E}-04$ & $1.65 \mathrm{E}-04$ & $0.00 \mathrm{E}+00$ & $0.00 \mathrm{E}+00$ & $0.00 \mathrm{E}+00$ & $8.00 \mathrm{E}-04$ \\
\hline $25 \%$ & $1.97 \mathrm{E}-06$ & $1.40 \mathrm{E}-04$ & $1.10 \mathrm{E}-13$ & $3.73 \mathrm{E}-04$ & $1.84 \mathrm{E}-04$ & $0.00 \mathrm{E}+00$ & $0.00 \mathrm{E}+00$ & $0.00 \mathrm{E}+00$ & 8.37E-04 \\
\hline $30 \%$ & $1.98 \mathrm{E}-06$ & $1.51 \mathrm{E}-04$ & $1.19 \mathrm{E}-13$ & $4.03 \mathrm{E}-04$ & $2.02 \mathrm{E}-04$ & $0.00 \mathrm{E}+00$ & $0.00 \mathrm{E}+00$ & $0.00 \mathrm{E}+00$ & 8.72E-04 \\
\hline $35 \%$ & $1.99 \mathrm{E}-06$ & $1.61 \mathrm{E}-04$ & $1.29 \mathrm{E}-13$ & 4.33E-04 & 2.19E-04 & $0.00 \mathrm{E}+00$ & $0.00 \mathrm{E}+00$ & $0.00 \mathrm{E}+00$ & 9.09E-04 \\
\hline $40 \%$ & $1.99 \mathrm{E}-06$ & $1.72 \mathrm{E}-04$ & $1.39 \mathrm{E}-13$ & 4.64E-04 & $2.35 \mathrm{E}-04$ & $0.00 \mathrm{E}+00$ & $0.00 \mathrm{E}+00$ & $0.00 \mathrm{E}+00$ & $9.42 \mathrm{E}-04$ \\
\hline $45 \%$ & $2.00 \mathrm{E}-06$ & $1.81 \mathrm{E}-04$ & $1.49 \mathrm{E}-13$ & $4.95 \mathrm{E}-04$ & $2.51 \mathrm{E}-04$ & $0.00 \mathrm{E}+00$ & $0.00 \mathrm{E}+00$ & $0.00 \mathrm{E}+00$ & $9.83 \mathrm{E}-04$ \\
\hline $50 \%$ & $2.00 \mathrm{E}-06$ & $1.91 \mathrm{E}-04$ & $1.59 \mathrm{E}-13$ & $5.23 \mathrm{E}-04$ & 2.64E-04 & $0.00 \mathrm{E}+00$ & $0.00 \mathrm{E}+00$ & $0.00 \mathrm{E}+00$ & $1.02 \mathrm{E}-03$ \\
\hline $55 \%$ & $2.00 \mathrm{E}-06$ & $2.03 \mathrm{E}-04$ & $1.69 \mathrm{E}-13$ & $5.61 \mathrm{E}-04$ & $2.77 \mathrm{E}-04$ & $0.00 \mathrm{E}+00$ & $0.00 \mathrm{E}+00$ & $0.00 \mathrm{E}+00$ & $1.06 \mathrm{E}-03$ \\
\hline $60 \%$ & $2.00 \mathrm{E}-06$ & 2.19E-04 & $1.81 \mathrm{E}-13$ & $5.98 \mathrm{E}-04$ & $2.92 \mathrm{E}-04$ & $0.00 \mathrm{E}+00$ & $0.00 \mathrm{E}+00$ & $0.00 \mathrm{E}+00$ & $1.11 \mathrm{E}-03$ \\
\hline $65 \%$ & $2.00 \mathrm{E}-06$ & $2.35 \mathrm{E}-04$ & $1.91 \mathrm{E}-13$ & $6.40 \mathrm{E}-04$ & $3.06 \mathrm{E}-04$ & $0.00 \mathrm{E}+00$ & $0.00 \mathrm{E}+00$ & $0.00 \mathrm{E}+00$ & $1.15 \mathrm{E}-03$ \\
\hline $70 \%$ & $2.00 \mathrm{E}-06$ & $2.51 \mathrm{E}-04$ & $2.06 \mathrm{E}-13$ & $6.86 \mathrm{E}-04$ & $3.23 \mathrm{E}-04$ & $0.00 \mathrm{E}+00$ & $0.00 \mathrm{E}+00$ & $0.00 \mathrm{E}+00$ & $1.20 \mathrm{E}-03$ \\
\hline $75 \%$ & $2.00 \mathrm{E}-06$ & $2.71 \mathrm{E}-04$ & $2.22 \mathrm{E}-13$ & 7.29E-04 & $3.41 \mathrm{E}-04$ & $0.00 \mathrm{E}+00$ & $0.00 \mathrm{E}+00$ & $0.00 \mathrm{E}+00$ & $1.25 \mathrm{E}-03$ \\
\hline $80 \%$ & $2.00 \mathrm{E}-06$ & $2.97 \mathrm{E}-04$ & $2.42 \mathrm{E}-13$ & $7.91 \mathrm{E}-04$ & $3.61 \mathrm{E}-04$ & $0.00 \mathrm{E}+00$ & $0.00 \mathrm{E}+00$ & $0.00 \mathrm{E}+00$ & $1.32 \mathrm{E}-03$ \\
\hline $85 \%$ & $2.00 \mathrm{E}-06$ & $3.34 \mathrm{E}-04$ & $2.66 \mathrm{E}-13$ & $8.60 \mathrm{E}-04$ & $3.83 \mathrm{E}-04$ & $0.00 \mathrm{E}+00$ & $0.00 \mathrm{E}+00$ & $0.00 \mathrm{E}+00$ & $1.40 \mathrm{E}-03$ \\
\hline $90 \%$ & $2.00 \mathrm{E}-06$ & $3.78 \mathrm{E}-04$ & $3.06 \mathrm{E}-13$ & $9.42 \mathrm{E}-04$ & 4.11E-04 & $0.00 \mathrm{E}+00$ & $0.00 \mathrm{E}+00$ & $0.00 \mathrm{E}+00$ & 1.49E-03 \\
\hline $95 \%$ & $2.00 \mathrm{E}-06$ & $4.44 \mathrm{E}-04$ & $3.63 \mathrm{E}-13$ & $1.07 \mathrm{E}-03$ & 4.47E-04 & $0.00 \mathrm{E}+00$ & $0.00 \mathrm{E}+00$ & $0.00 \mathrm{E}+00$ & $1.61 \mathrm{E}-03$ \\
\hline Mean & $1.95 \mathrm{E}-06$ & $2.20 \mathrm{E}-04$ & $1.78 \mathrm{E}-13$ & $5.73 \mathrm{E}-04$ & 2.63E-04 & $1.47 \mathrm{E}-10$ & $1.02 \mathrm{E}-09$ & $1.56 \mathrm{E}-12$ & $1.06 \mathrm{E}-03$ \\
\hline Min & $3.28 \mathrm{E}-10$ & $1.23 \mathrm{E}-09$ & $2.67 \mathrm{E}-15$ & $9.70 \mathrm{E}-09$ & $1.89 \mathrm{E}-09$ & $0.00 \mathrm{E}+00$ & $0.00 \mathrm{E}+00$ & $0.00 \mathrm{E}+00$ & $1.32 \mathrm{E}-08$ \\
\hline Max & $2.00 \mathrm{E}-06$ & $1.55 \mathrm{E}-03$ & $1.03 \mathrm{E}-12$ & $1.95 \mathrm{E}-03$ & $5.32 \mathrm{E}-04$ & $2.86 \mathrm{E}-07$ & $2.84 \mathrm{E}-06$ & $2.77 \mathrm{E}-09$ & $2.49 \mathrm{E}-03$ \\
\hline
\end{tabular}


TABLE C.22 Peak DSR Percentiles (mrem/yr per pCi/g) for Different Exposure Pathways for Pu-239 in Recreational Use Scenario

\begin{tabular}{|c|c|c|c|c|c|c|c|c|}
\hline Percentile & External & $\begin{array}{c}\text { Inhalation } \\
\text { w/o Rn }\end{array}$ & $\begin{array}{c}\text { Meat } \\
\text { Ingestion } \\
(\mathrm{WI})\end{array}$ & $\begin{array}{c}\text { Soil } \\
\text { Ingestion }\end{array}$ & $\begin{array}{c}\text { Water } \\
\text { Ingestion }\end{array}$ & $\begin{array}{l}\text { Aquatic } \\
\text { Foods }\end{array}$ & $\begin{array}{c}\text { Meat } \\
\text { Ingestion } \\
\text { (WD) }\end{array}$ & $\begin{array}{c}\text { All } \\
\text { Pathways }\end{array}$ \\
\hline $5 \%$ & $8.56 \mathrm{E}-06$ & $1.81 \mathrm{E}-04$ & $5.41 \mathrm{E}-04$ & $1.91 \mathrm{E}-04$ & $0.00 \mathrm{E}+00$ & $0.00 \mathrm{E}+00$ & $0.00 \mathrm{E}+00$ & $1.46 \mathrm{E}-03$ \\
\hline $10 \%$ & $9.10 \mathrm{E}-06$ & $2.48 \mathrm{E}-04$ & $6.58 \mathrm{E}-04$ & $2.77 \mathrm{E}-04$ & $0.00 \mathrm{E}+00$ & $0.00 \mathrm{E}+00$ & $0.00 \mathrm{E}+00$ & $1.66 \mathrm{E}-03$ \\
\hline $15 \%$ & $9.32 \mathrm{E}-06$ & $2.84 \mathrm{E}-04$ & 7.39E-04 & $3.41 \mathrm{E}-04$ & $0.00 \mathrm{E}+00$ & $0.00 \mathrm{E}+00$ & $0.00 \mathrm{E}+00$ & $1.80 \mathrm{E}-03$ \\
\hline $20 \%$ & $9.41 \mathrm{E}-06$ & $3.14 \mathrm{E}-04$ & $8.17 \mathrm{E}-04$ & $3.94 \mathrm{E}-04$ & $0.00 \mathrm{E}+00$ & $0.00 \mathrm{E}+00$ & $0.00 \mathrm{E}+00$ & $1.92 \mathrm{E}-03$ \\
\hline $25 \%$ & $9.48 \mathrm{E}-06$ & 3.39E-04 & 8.91E-04 & $4.42 \mathrm{E}-04$ & $0.00 \mathrm{E}+00$ & $0.00 \mathrm{E}+00$ & $0.00 \mathrm{E}+00$ & $2.02 \mathrm{E}-03$ \\
\hline $30 \%$ & $9.51 \mathrm{E}-06$ & $3.66 \mathrm{E}-04$ & $9.61 \mathrm{E}-04$ & $4.83 \mathrm{E}-04$ & $0.00 \mathrm{E}+00$ & $0.00 \mathrm{E}+00$ & $0.00 \mathrm{E}+00$ & $2.10 \mathrm{E}-03$ \\
\hline $35 \%$ & $9.54 \mathrm{E}-06$ & $3.89 \mathrm{E}-04$ & $1.03 \mathrm{E}-03$ & $5.24 \mathrm{E}-04$ & $0.00 \mathrm{E}+00$ & $0.00 \mathrm{E}+00$ & $0.00 \mathrm{E}+00$ & $2.19 \mathrm{E}-03$ \\
\hline $40 \%$ & $9.56 \mathrm{E}-06$ & 4.13E-04 & $1.11 \mathrm{E}-03$ & $5.63 \mathrm{E}-04$ & $0.00 \mathrm{E}+00$ & $0.00 \mathrm{E}+00$ & $0.00 \mathrm{E}+00$ & $2.27 \mathrm{E}-03$ \\
\hline $45 \%$ & $9.58 \mathrm{E}-06$ & $4.36 \mathrm{E}-04$ & $1.18 \mathrm{E}-03$ & $5.96 \mathrm{E}-04$ & $0.00 \mathrm{E}+00$ & $0.00 \mathrm{E}+00$ & $0.00 \mathrm{E}+00$ & $2.36 \mathrm{E}-03$ \\
\hline $50 \%$ & $9.59 \mathrm{E}-06$ & 4.61E-04 & $1.26 \mathrm{E}-03$ & $6.31 \mathrm{E}-04$ & $0.00 \mathrm{E}+00$ & $0.00 \mathrm{E}+00$ & $0.00 \mathrm{E}+00$ & $2.45 \mathrm{E}-03$ \\
\hline $55 \%$ & $9.60 \mathrm{E}-06$ & 4.94E-04 & $1.35 \mathrm{E}-03$ & $6.64 \mathrm{E}-04$ & $0.00 \mathrm{E}+00$ & $0.00 \mathrm{E}+00$ & $0.00 \mathrm{E}+00$ & $2.54 \mathrm{E}-03$ \\
\hline $60 \%$ & $9.60 \mathrm{E}-06$ & $5.28 \mathrm{E}-04$ & $1.43 \mathrm{E}-03$ & $7.00 \mathrm{E}-04$ & $0.00 \mathrm{E}+00$ & $0.00 \mathrm{E}+00$ & $0.00 \mathrm{E}+00$ & $2.65 \mathrm{E}-03$ \\
\hline $65 \%$ & $9.61 \mathrm{E}-06$ & $5.66 \mathrm{E}-04$ & $1.53 \mathrm{E}-03$ & $7.36 \mathrm{E}-04$ & $0.00 \mathrm{E}+00$ & $0.00 \mathrm{E}+00$ & $0.00 \mathrm{E}+00$ & $2.75 \mathrm{E}-03$ \\
\hline $70 \%$ & $9.61 \mathrm{E}-06$ & $6.06 \mathrm{E}-04$ & $1.64 \mathrm{E}-03$ & 7.72E-04 & $0.00 \mathrm{E}+00$ & $0.00 \mathrm{E}+00$ & $0.00 \mathrm{E}+00$ & $2.87 \mathrm{E}-03$ \\
\hline $75 \%$ & $9.62 \mathrm{E}-06$ & $6.52 \mathrm{E}-04$ & $1.75 \mathrm{E}-03$ & $8.16 \mathrm{E}-04$ & $0.00 \mathrm{E}+00$ & $0.00 \mathrm{E}+00$ & $0.00 \mathrm{E}+00$ & $3.02 \mathrm{E}-03$ \\
\hline $80 \%$ & $9.62 \mathrm{E}-06$ & $7.13 \mathrm{E}-04$ & $1.90 \mathrm{E}-03$ & $8.65 \mathrm{E}-04$ & $0.00 \mathrm{E}+00$ & $0.00 \mathrm{E}+00$ & $0.00 \mathrm{E}+00$ & $3.18 \mathrm{E}-03$ \\
\hline $85 \%$ & $9.62 \mathrm{E}-06$ & $8.00 \mathrm{E}-04$ & $2.06 \mathrm{E}-03$ & $9.22 \mathrm{E}-04$ & $0.00 \mathrm{E}+00$ & $0.00 \mathrm{E}+00$ & $0.00 \mathrm{E}+00$ & $3.36 \mathrm{E}-03$ \\
\hline $90 \%$ & $9.62 \mathrm{E}-06$ & $9.02 \mathrm{E}-04$ & $2.26 \mathrm{E}-03$ & $9.87 \mathrm{E}-04$ & $0.00 \mathrm{E}+00$ & $0.00 \mathrm{E}+00$ & $0.00 \mathrm{E}+00$ & $3.56 \mathrm{E}-03$ \\
\hline $95 \%$ & $9.62 \mathrm{E}-06$ & $1.06 \mathrm{E}-03$ & $2.56 \mathrm{E}-03$ & $1.08 \mathrm{E}-03$ & $0.00 \mathrm{E}+00$ & $0.00 \mathrm{E}+00$ & $0.00 \mathrm{E}+00$ & $3.87 \mathrm{E}-03$ \\
\hline Mean & $9.38 \mathrm{E}-06$ & $5.30 \mathrm{E}-04$ & $1.37 \mathrm{E}-03$ & $6.31 \mathrm{E}-04$ & $1.82 \mathrm{E}-14$ & $2.24 \mathrm{E}-13$ & $3.38 \mathrm{E}-16$ & $2.54 \mathrm{E}-03$ \\
\hline Min & $6.72 \mathrm{E}-08$ & $1.24 \mathrm{E}-06$ & $1.23 \mathrm{E}-05$ & $2.42 \mathrm{E}-06$ & $0.00 \mathrm{E}+00$ & $0.00 \mathrm{E}+00$ & $0.00 \mathrm{E}+00$ & $1.82 \mathrm{E}-05$ \\
\hline $\operatorname{Max}$ & $9.62 \mathrm{E}-06$ & $3.74 \mathrm{E}-03$ & 4.63E- 03 & $1.29 \mathrm{E}-03$ & $2.78 \mathrm{E}-11$ & 4.04E-10 & 7.19E-13 & $6.03 \mathrm{E}-03$ \\
\hline
\end{tabular}


TABLE C.23 Peak DSR Percentiles (mrem/yr per pCi/g) for Different Exposure Pathways for Sr-90 in Recreational Use Scenario

\begin{tabular}{|c|c|c|c|c|c|c|c|c|}
\hline Percentile & External & $\begin{array}{c}\text { Inhalation } \\
\text { w/o Rn }\end{array}$ & $\begin{array}{c}\text { Meat } \\
\text { Ingestion } \\
(\mathrm{WI})\end{array}$ & $\begin{array}{c}\text { Soil } \\
\text { Ingestion }\end{array}$ & $\begin{array}{c}\text { Water } \\
\text { Ingestion }\end{array}$ & $\begin{array}{l}\text { Aquatic } \\
\text { Foods }\end{array}$ & $\begin{array}{c}\text { Meat } \\
\text { Ingestion } \\
\text { (WD) }\end{array}$ & $\begin{array}{c}\text { All } \\
\text { Pathways }\end{array}$ \\
\hline $5 \%$ & $1.57 \mathrm{E}-06$ & $6.82 \mathrm{E}-10$ & $1.07 \mathrm{E}-04$ & $8.55 \mathrm{E}-08$ & $0.00 \mathrm{E}+00$ & $0.00 \mathrm{E}+00$ & $0.00 \mathrm{E}+00$ & $1.18 \mathrm{E}-04$ \\
\hline $10 \%$ & 1.49E-05 & $5.84 \mathrm{E}-09$ & $8.34 \mathrm{E}-04$ & $5.86 \mathrm{E}-07$ & $0.00 \mathrm{E}+00$ & $0.00 \mathrm{E}+00$ & $0.00 \mathrm{E}+00$ & $9.18 \mathrm{E}-04$ \\
\hline $15 \%$ & $3.59 \mathrm{E}-05$ & 1.19E-08 & $1.57 \mathrm{E}-03$ & $1.24 \mathrm{E}-06$ & $0.00 \mathrm{E}+00$ & $0.00 \mathrm{E}+00$ & $0.00 \mathrm{E}+00$ & $1.64 \mathrm{E}-03$ \\
\hline $20 \%$ & $5.40 \mathrm{E}-05$ & $1.75 \mathrm{E}-08$ & $2.21 \mathrm{E}-03$ & $1.87 \mathrm{E}-06$ & $0.00 \mathrm{E}+00$ & $0.00 \mathrm{E}+00$ & $0.00 \mathrm{E}+00$ & $2.31 \mathrm{E}-03$ \\
\hline $25 \%$ & 7.09E-05 & $2.30 \mathrm{E}-08$ & $2.78 \mathrm{E}-03$ & $2.45 \mathrm{E}-06$ & $0.00 \mathrm{E}+00$ & $0.00 \mathrm{E}+00$ & $0.00 \mathrm{E}+00$ & $2.88 \mathrm{E}-03$ \\
\hline $30 \%$ & $8.41 \mathrm{E}-05$ & $2.77 \mathrm{E}-08$ & $3.40 \mathrm{E}-03$ & $3.04 \mathrm{E}-06$ & $0.00 \mathrm{E}+00$ & $0.00 \mathrm{E}+00$ & $0.00 \mathrm{E}+00$ & $3.51 \mathrm{E}-03$ \\
\hline $35 \%$ & $9.46 \mathrm{E}-05$ & $3.21 \mathrm{E}-08$ & $4.11 \mathrm{E}-03$ & $3.60 \mathrm{E}-06$ & $0.00 \mathrm{E}+00$ & $0.00 \mathrm{E}+00$ & $0.00 \mathrm{E}+00$ & $4.23 \mathrm{E}-03$ \\
\hline $40 \%$ & $1.03 \mathrm{E}-04$ & $3.53 \mathrm{E}-08$ & 4.78E-03 & $4.14 \mathrm{E}-06$ & $0.00 \mathrm{E}+00$ & $0.00 \mathrm{E}+00$ & $0.00 \mathrm{E}+00$ & 4.93E-03 \\
\hline $45 \%$ & $1.09 \mathrm{E}-04$ & $3.88 \mathrm{E}-08$ & $5.55 \mathrm{E}-03$ & $4.62 \mathrm{E}-06$ & $0.00 \mathrm{E}+00$ & $0.00 \mathrm{E}+00$ & $0.00 \mathrm{E}+00$ & $5.69 \mathrm{E}-03$ \\
\hline $50 \%$ & $1.14 \mathrm{E}-04$ & $4.22 \mathrm{E}-08$ & $6.44 \mathrm{E}-03$ & $5.14 \mathrm{E}-06$ & $0.00 \mathrm{E}+00$ & $0.00 \mathrm{E}+00$ & $0.00 \mathrm{E}+00$ & $6.56 \mathrm{E}-03$ \\
\hline $55 \%$ & $1.19 \mathrm{E}-04$ & 4.57E-08 & $7.55 \mathrm{E}-03$ & $5.55 \mathrm{E}-06$ & $0.00 \mathrm{E}+00$ & $0.00 \mathrm{E}+00$ & $0.00 \mathrm{E}+00$ & $7.69 \mathrm{E}-03$ \\
\hline $60 \%$ & $1.22 \mathrm{E}-04$ & 4.94E-08 & $9.10 \mathrm{E}-03$ & $6.03 \mathrm{E}-06$ & $0.00 \mathrm{E}+00$ & $0.00 \mathrm{E}+00$ & $0.00 \mathrm{E}+00$ & $9.24 \mathrm{E}-03$ \\
\hline $65 \%$ & $1.25 \mathrm{E}-04$ & $5.36 \mathrm{E}-08$ & $1.07 \mathrm{E}-02$ & $6.52 \mathrm{E}-06$ & $0.00 \mathrm{E}+00$ & $0.00 \mathrm{E}+00$ & $0.00 \mathrm{E}+00$ & $1.08 \mathrm{E}-02$ \\
\hline $70 \%$ & $1.28 \mathrm{E}-04$ & $5.82 \mathrm{E}-08$ & $1.27 \mathrm{E}-02$ & $7.00 \mathrm{E}-06$ & $0.00 \mathrm{E}+00$ & $0.00 \mathrm{E}+00$ & $0.00 \mathrm{E}+00$ & $1.28 \mathrm{E}-02$ \\
\hline $75 \%$ & $1.30 \mathrm{E}-04$ & $6.41 \mathrm{E}-08$ & $1.52 \mathrm{E}-02$ & $7.50 \mathrm{E}-06$ & $0.00 \mathrm{E}+00$ & $0.00 \mathrm{E}+00$ & $0.00 \mathrm{E}+00$ & $1.54 \mathrm{E}-02$ \\
\hline $80 \%$ & $1.31 \mathrm{E}-04$ & 7.04E-08 & $1.85 \mathrm{E}-02$ & $8.06 \mathrm{E}-06$ & $0.00 \mathrm{E}+00$ & $0.00 \mathrm{E}+00$ & $0.00 \mathrm{E}+00$ & $1.86 \mathrm{E}-02$ \\
\hline $85 \%$ & $1.33 \mathrm{E}-04$ & 7.87E-08 & $2.33 \mathrm{E}-02$ & $8.76 \mathrm{E}-06$ & $0.00 \mathrm{E}+00$ & $0.00 \mathrm{E}+00$ & $0.00 \mathrm{E}+00$ & $2.34 \mathrm{E}-02$ \\
\hline $90 \%$ & $1.33 \mathrm{E}-04$ & 8.97E-08 & $3.08 \mathrm{E}-02$ & $9.72 \mathrm{E}-06$ & $0.00 \mathrm{E}+00$ & $0.00 \mathrm{E}+00$ & $0.00 \mathrm{E}+00$ & $3.09 \mathrm{E}-02$ \\
\hline $95 \%$ & $1.34 \mathrm{E}-04$ & $1.13 \mathrm{E}-07$ & 4.74E-02 & $1.07 \mathrm{E}-05$ & $0.00 \mathrm{E}+00$ & $0.00 \mathrm{E}+00$ & $0.00 \mathrm{E}+00$ & $4.75 \mathrm{E}-02$ \\
\hline Mean & $9.53 \mathrm{E}-05$ & $4.72 \mathrm{E}-08$ & $1.34 \mathrm{E}-02$ & $5.15 \mathrm{E}-06$ & $2.21 \mathrm{E}-07$ & $1.35 \mathrm{E}-05$ & $5.66 \mathrm{E}-08$ & $1.35 \mathrm{E}-02$ \\
\hline Min & $0.00 \mathrm{E}+00$ & $0.00 \mathrm{E}+00$ & $4.49 \mathrm{E}-29$ & $0.00 \mathrm{E}+00$ & $0.00 \mathrm{E}+00$ & $0.00 \mathrm{E}+00$ & $0.00 \mathrm{E}+00$ & $4.49 \mathrm{E}-29$ \\
\hline Max & $1.35 \mathrm{E}-04$ & $4.56 \mathrm{E}-07$ & $5.62 \mathrm{E}-01$ & $1.43 \mathrm{E}-05$ & $1.01 \mathrm{E}-04$ & $2.47 \mathrm{E}-02$ & $2.84 \mathrm{E}-05$ & $5.62 \mathrm{E}-01$ \\
\hline
\end{tabular}


TABLE C.24 Peak DSR Percentiles (mrem/yr per pCi/g) for Different Exposure Pathways for Tc-99 in Recreational Use Scenario

\begin{tabular}{|c|c|c|c|c|c|c|c|c|}
\hline Percentile & External & $\begin{array}{c}\text { Inhalation } \\
\text { w/o Rn }\end{array}$ & $\begin{array}{c}\text { Meat } \\
\text { Ingestion } \\
\text { (WI) }\end{array}$ & $\begin{array}{c}\text { Soil } \\
\text { Ingestion }\end{array}$ & $\begin{array}{c}\text { Water } \\
\text { Ingestion }\end{array}$ & $\begin{array}{l}\text { Aquatic } \\
\text { Foods }\end{array}$ & $\begin{array}{c}\text { Meat } \\
\text { Ingestion } \\
\text { (WD) }\end{array}$ & $\begin{array}{c}\text { All } \\
\text { Pathways }\end{array}$ \\
\hline $5 \%$ & $1.05 \mathrm{E}-13$ & $1.14 \mathrm{E}-15$ & $6.12 \mathrm{E}-12$ & $3.41 \mathrm{E}-14$ & 7.79E-07 & $4.17 \mathrm{E}-06$ & 1.41E-09 & $5.78 \mathrm{E}-06$ \\
\hline $10 \%$ & $2.34 \mathrm{E}-13$ & $2.81 \mathrm{E}-15$ & $1.57 \mathrm{E}-11$ & $8.11 \mathrm{E}-14$ & $1.76 \mathrm{E}-06$ & $1.30 \mathrm{E}-05$ & 4.07E-09 & $1.69 \mathrm{E}-05$ \\
\hline $15 \%$ & $4.25 \mathrm{E}-13$ & $5.20 \mathrm{E}-15$ & 3.34E-11 & $1.58 \mathrm{E}-13$ & $3.16 \mathrm{E}-06$ & $2.78 \mathrm{E}-05$ & 7.40E-09 & $3.34 \mathrm{E}-05$ \\
\hline $20 \%$ & $7.26 \mathrm{E}-13$ & $9.21 \mathrm{E}-15$ & $6.30 \mathrm{E}-11$ & $2.67 \mathrm{E}-13$ & $4.95 \mathrm{E}-06$ & $4.95 \mathrm{E}-05$ & $1.23 \mathrm{E}-08$ & $5.84 \mathrm{E}-05$ \\
\hline $25 \%$ & $1.24 \mathrm{E}-12$ & $1.62 \mathrm{E}-14$ & $1.12 \mathrm{E}-10$ & $4.43 \mathrm{E}-13$ & 7.74E-06 & $8.31 \mathrm{E}-05$ & $1.93 \mathrm{E}-08$ & $9.76 \mathrm{E}-05$ \\
\hline $30 \%$ & $1.97 \mathrm{E}-12$ & $2.61 \mathrm{E}-14$ & $1.88 \mathrm{E}-10$ & $7.09 \mathrm{E}-13$ & $1.17 \mathrm{E}-05$ & $1.25 \mathrm{E}-04$ & $3.05 \mathrm{E}-08$ & $1.50 \mathrm{E}-04$ \\
\hline $35 \%$ & $3.16 \mathrm{E}-12$ & $4.17 \mathrm{E}-14$ & $3.04 \mathrm{E}-10$ & $1.17 \mathrm{E}-12$ & $1.78 \mathrm{E}-05$ & $1.80 \mathrm{E}-04$ & $4.51 \mathrm{E}-08$ & $2.16 \mathrm{E}-04$ \\
\hline $40 \%$ & $5.01 \mathrm{E}-12$ & $6.51 \mathrm{E}-14$ & $5.03 \mathrm{E}-10$ & $1.87 \mathrm{E}-12$ & $2.68 \mathrm{E}-05$ & $2.79 \mathrm{E}-04$ & $6.95 \mathrm{E}-08$ & $3.41 \mathrm{E}-04$ \\
\hline $45 \%$ & $8.06 \mathrm{E}-12$ & $1.05 \mathrm{E}-13$ & $9.03 \mathrm{E}-10$ & $3.06 \mathrm{E}-12$ & $3.89 \mathrm{E}-05$ & 3.97E-04 & $9.58 \mathrm{E}-08$ & $4.72 \mathrm{E}-04$ \\
\hline $50 \%$ & $1.36 \mathrm{E}-11$ & $1.80 \mathrm{E}-13$ & $1.63 \mathrm{E}-09$ & $5.11 \mathrm{E}-12$ & $5.52 \mathrm{E}-05$ & $5.40 \mathrm{E}-04$ & $1.30 \mathrm{E}-07$ & $6.48 \mathrm{E}-04$ \\
\hline $55 \%$ & $2.45 \mathrm{E}-11$ & $3.38 \mathrm{E}-13$ & $3.26 \mathrm{E}-09$ & $9.62 \mathrm{E}-12$ & $7.81 \mathrm{E}-05$ & 7.46E-04 & $1.71 \mathrm{E}-07$ & $8.73 \mathrm{E}-04$ \\
\hline $60 \%$ & $5.72 \mathrm{E}-11$ & $8.07 \mathrm{E}-13$ & $1.14 \mathrm{E}-08$ & $2.25 \mathrm{E}-11$ & $1.06 \mathrm{E}-04$ & $1.02 \mathrm{E}-03$ & $2.28 \mathrm{E}-07$ & $1.17 \mathrm{E}-03$ \\
\hline $65 \%$ & $3.76 \mathrm{E}-10$ & $6.00 \mathrm{E}-12$ & $1.67 \mathrm{E}-07$ & $1.50 \mathrm{E}-10$ & $1.41 \mathrm{E}-04$ & $1.33 \mathrm{E}-03$ & $2.88 \mathrm{E}-07$ & $1.50 \mathrm{E}-03$ \\
\hline $70 \%$ & $3.75 \mathrm{E}-06$ & $2.93 \mathrm{E}-08$ & $7.50 \mathrm{E}-05$ & 8.08E-07 & 1.79E-04 & $1.76 \mathrm{E}-03$ & $3.52 \mathrm{E}-07$ & $1.93 \mathrm{E}-03$ \\
\hline $75 \%$ & $3.77 \mathrm{E}-06$ & $3.75 \mathrm{E}-08$ & $1.44 \mathrm{E}-04$ & $1.18 \mathrm{E}-06$ & $2.11 \mathrm{E}-04$ & $2.30 \mathrm{E}-03$ & 4.30E-07 & $2.49 \mathrm{E}-03$ \\
\hline $80 \%$ & 3.79E-06 & $4.50 \mathrm{E}-08$ & $2.21 \mathrm{E}-04$ & $1.47 \mathrm{E}-06$ & 2.33E-04 & $3.10 \mathrm{E}-03$ & 5.34E-07 & $3.31 \mathrm{E}-03$ \\
\hline $85 \%$ & $3.80 \mathrm{E}-06$ & $5.33 \mathrm{E}-08$ & $3.27 \mathrm{E}-04$ & $1.69 \mathrm{E}-06$ & $2.50 \mathrm{E}-04$ & $4.16 \mathrm{E}-03$ & 6.64E-07 & $4.36 \mathrm{E}-03$ \\
\hline $90 \%$ & $3.81 \mathrm{E}-06$ & $6.56 \mathrm{E}-08$ & 4.93E-04 & $1.96 \mathrm{E}-06$ & $2.65 \mathrm{E}-04$ & $6.14 \mathrm{E}-03$ & $8.70 \mathrm{E}-07$ & $6.31 \mathrm{E}-03$ \\
\hline $95 \%$ & $3.82 \mathrm{E}-06$ & $8.47 \mathrm{E}-08$ & $8.44 \mathrm{E}-04$ & $2.32 \mathrm{E}-06$ & $2.81 \mathrm{E}-04$ & $1.05 \mathrm{E}-02$ & $1.25 \mathrm{E}-06$ & $1.07 \mathrm{E}-02$ \\
\hline Mean & $1.30 \mathrm{E}-06$ & $1.94 \mathrm{E}-08$ & $1.62 \mathrm{E}-04$ & $5.45 \mathrm{E}-07$ & $1.03 \mathrm{E}-04$ & $2.46 \mathrm{E}-03$ & $3.29 \mathrm{E}-07$ & $2.56 \mathrm{E}-03$ \\
\hline Min & $1.71 \mathrm{E}-15$ & $1.79 \mathrm{E}-17$ & $5.18 \mathrm{E}-14$ & $8.70 \mathrm{E}-16$ & $0.00 \mathrm{E}+00$ & $0.00 \mathrm{E}+00$ & $0.00 \mathrm{E}+00$ & $1.18 \mathrm{E}-09$ \\
\hline Max & $3.86 \mathrm{E}-06$ & $2.43 \mathrm{E}-07$ & $1.15 \mathrm{E}-02$ & $3.12 \mathrm{E}-06$ & $3.60 \mathrm{E}-04$ & $1.28 \mathrm{E}-01$ & 7.23E-06 & $1.29 \mathrm{E}-01$ \\
\hline
\end{tabular}

Tables C.25-C.32 show the peak DSR percentiles (mrem/yr per pCi/g) for different exposure pathways for individual radionuclides in the onsite resident scenario. RESRAD (onsite) version 6.5 was used in the analysis. For C-14 and Tc-99, water-dependent pathways were dominant, and for others (Am-241, Ba-133, Cs-137, Pu-238, Pu-239, and Sr-90), waterindependent pathways were dominant. For Am-241, plant ingestion, external exposure, and soil ingestion were dominant. For Ba-133, external exposure was dominant. For C-14, aquatic food ingestion resulted in most dose. For Cs-137, external exposure and meat ingestion resulted in most dose. For $\mathrm{Pu}-238$ and $\mathrm{Pu}-239$, plant ingestion, soil ingestion, and inhalation were dominant exposure pathways. For Sr-90, plant and meat ingestion resulted in most dose. For Tc-99, water and plant ingestion resulted in most dose. 
TABLE C.25 Peak DSR Percentiles (mrem/yr per pCi/g) for Different Exposure Pathways for Am-241 in Onsite Resident Scenario

\begin{tabular}{|c|c|c|c|c|c|c|c|c|c|c|c|c|}
\hline Percentile & External & $\begin{array}{c}\text { Inhalation } \\
\text { w/o Rn }\end{array}$ & $\begin{array}{c}\text { Plant } \\
\text { Ingestion } \\
(\mathrm{WI})\end{array}$ & $\begin{array}{c}\text { Meat } \\
\text { Ingestion } \\
(\mathrm{WI})\end{array}$ & $\begin{array}{c}\text { Milk } \\
\text { Ingestion } \\
(\mathrm{WI})\end{array}$ & $\begin{array}{c}\text { Soil } \\
\text { Ingestion }\end{array}$ & $\begin{array}{c}\text { Water } \\
\text { Ingestion }\end{array}$ & $\begin{array}{l}\text { Aquatic } \\
\text { Foods }\end{array}$ & $\begin{array}{c}\text { Plant } \\
\text { Ingestion } \\
\text { (WD) }\end{array}$ & $\begin{array}{c}\text { Meat } \\
\text { Ingestion } \\
\text { (WD) }\end{array}$ & $\begin{array}{c}\text { Milk } \\
\text { Ingestion } \\
\text { (WD) }\end{array}$ & $\begin{array}{c}\text { All } \\
\text { Pathways }\end{array}$ \\
\hline $5 \%$ & $4.45 \mathrm{E}-03$ & $6.67 \mathrm{E}-04$ & $1.13 \mathrm{E}-02$ & $3.75 \mathrm{E}-04$ & $9.85 \mathrm{E}-06$ & $2.50 \mathrm{E}-03$ & $0.00 \mathrm{E}+00$ & $0.00 \mathrm{E}+00$ & $0.00 \mathrm{E}+00$ & $0.00 \mathrm{E}+00$ & $0.00 \mathrm{E}+00$ & $2.96 \mathrm{E}-02$ \\
\hline $10 \%$ & $5.00 \mathrm{E}-03$ & $9.09 \mathrm{E}-04$ & $1.59 \mathrm{E}-02$ & 4.14E-04 & $1.28 \mathrm{E}-05$ & $3.66 \mathrm{E}-03$ & $0.00 \mathrm{E}+00$ & $0.00 \mathrm{E}+00$ & $0.00 \mathrm{E}+00$ & $0.00 \mathrm{E}+00$ & $0.00 \mathrm{E}+00$ & $3.61 \mathrm{E}-02$ \\
\hline $15 \%$ & $5.44 \mathrm{E}-03$ & $1.06 \mathrm{E}-03$ & $2.00 \mathrm{E}-02$ & $4.42 \mathrm{E}-04$ & $1.54 \mathrm{E}-05$ & $4.54 \mathrm{E}-03$ & $0.00 \mathrm{E}+00$ & $0.00 \mathrm{E}+00$ & $0.00 \mathrm{E}+00$ & $0.00 \mathrm{E}+00$ & $0.00 \mathrm{E}+00$ & $4.05 \mathrm{E}-02$ \\
\hline $20 \%$ & $5.84 \mathrm{E}-03$ & $1.20 \mathrm{E}-03$ & $2.40 \mathrm{E}-02$ & 4.64E-04 & $1.78 \mathrm{E}-05$ & 5.29E-03 & $0.00 \mathrm{E}+00$ & $0.00 \mathrm{E}+00$ & $0.00 \mathrm{E}+00$ & $0.00 \mathrm{E}+00$ & $0.00 \mathrm{E}+00$ & $4.48 \mathrm{E}-02$ \\
\hline $30 \%$ & $6.53 \mathrm{E}-03$ & $1.53 \mathrm{E}-03$ & $3.24 \mathrm{E}-02$ & $5.04 \mathrm{E}-04$ & $2.24 \mathrm{E}-05$ & $6.55 \mathrm{E}-03$ & $0.00 \mathrm{E}+00$ & $0.00 \mathrm{E}+00$ & $0.00 \mathrm{E}+00$ & $0.00 \mathrm{E}+00$ & $0.00 \mathrm{E}+00$ & 5.33E-02 \\
\hline $35 \%$ & $6.87 \mathrm{E}-03$ & $1.70 \mathrm{E}-03$ & $3.67 \mathrm{E}-02$ & $5.23 \mathrm{E}-04$ & $2.46 \mathrm{E}-05$ & $7.10 \mathrm{E}-03$ & $0.00 \mathrm{E}+00$ & $0.00 \mathrm{E}+00$ & $0.00 \mathrm{E}+00$ & $0.00 \mathrm{E}+00$ & $0.00 \mathrm{E}+00$ & $5.75 \mathrm{E}-02$ \\
\hline $40 \%$ & 7.24E-03 & $1.85 \mathrm{E}-03$ & $4.16 \mathrm{E}-02$ & $5.40 \mathrm{E}-04$ & $2.70 \mathrm{E}-05$ & $7.61 \mathrm{E}-03$ & $0.00 \mathrm{E}+00$ & $0.00 \mathrm{E}+00$ & $0.00 \mathrm{E}+00$ & $0.00 \mathrm{E}+00$ & $0.00 \mathrm{E}+00$ & $6.29 \mathrm{E}-02$ \\
\hline $45 \%$ & $7.61 \mathrm{E}-03$ & $2.01 \mathrm{E}-03$ & $4.68 \mathrm{E}-02$ & $5.55 \mathrm{E}-04$ & $2.96 \mathrm{E}-05$ & $8.10 \mathrm{E}-03$ & $0.00 \mathrm{E}+00$ & $0.00 \mathrm{E}+00$ & $0.00 \mathrm{E}+00$ & $0.00 \mathrm{E}+00$ & $0.00 \mathrm{E}+00$ & $6.81 \mathrm{E}-02$ \\
\hline $50 \%$ & $8.00 \mathrm{E}-03$ & $2.18 \mathrm{E}-03$ & $5.24 \mathrm{E}-02$ & $5.72 \mathrm{E}-04$ & $3.22 \mathrm{E}-05$ & $8.57 \mathrm{E}-03$ & $0.00 \mathrm{E}+00$ & $0.00 \mathrm{E}+00$ & $0.00 \mathrm{E}+00$ & $0.00 \mathrm{E}+00$ & $0.00 \mathrm{E}+00$ & $7.46 \mathrm{E}-02$ \\
\hline $55 \%$ & $8.41 \mathrm{E}-03$ & $2.35 \mathrm{E}-03$ & $5.92 \mathrm{E}-02$ & $5.88 \mathrm{E}-04$ & $3.51 \mathrm{E}-05$ & $9.00 \mathrm{E}-03$ & $0.00 \mathrm{E}+00$ & $0.00 \mathrm{E}+00$ & $0.00 \mathrm{E}+00$ & $0.00 \mathrm{E}+00$ & $0.00 \mathrm{E}+00$ & 8.03E-02 \\
\hline $60 \%$ & $8.86 \mathrm{E}-03$ & $2.55 \mathrm{E}-03$ & $6.64 \mathrm{E}-02$ & $6.06 \mathrm{E}-04$ & $3.88 \mathrm{E}-05$ & $9.47 \mathrm{E}-03$ & $0.00 \mathrm{E}+00$ & $0.00 \mathrm{E}+00$ & $0.00 \mathrm{E}+00$ & $0.00 \mathrm{E}+00$ & $0.00 \mathrm{E}+00$ & $8.78 \mathrm{E}-02$ \\
\hline $65 \%$ & $9.40 \mathrm{E}-03$ & $2.76 \mathrm{E}-03$ & $7.46 \mathrm{E}-02$ & $6.28 \mathrm{E}-04$ & $4.24 \mathrm{E}-05$ & $9.98 \mathrm{E}-03$ & $0.00 \mathrm{E}+00$ & $0.00 \mathrm{E}+00$ & $0.00 \mathrm{E}+00$ & $0.00 \mathrm{E}+00$ & $0.00 \mathrm{E}+00$ & $9.62 \mathrm{E}-02$ \\
\hline $75 \%$ & $1.06 \mathrm{E}-02$ & $3.26 \mathrm{E}-03$ & $9.80 \mathrm{E}-02$ & $6.77 \mathrm{E}-04$ & $5.19 \mathrm{E}-05$ & $1.11 \mathrm{E}-02$ & $0.00 \mathrm{E}+00$ & $0.00 \mathrm{E}+00$ & $0.00 \mathrm{E}+00$ & $0.00 \mathrm{E}+00$ & $0.00 \mathrm{E}+00$ & $1.20 \mathrm{E}-01$ \\
\hline $80 \%$ & $1.14 \mathrm{E}-02$ & $3.59 \mathrm{E}-03$ & $1.14 \mathrm{E}-01$ & $7.03 \mathrm{E}-04$ & $5.92 \mathrm{E}-05$ & $1.18 \mathrm{E}-02$ & $0.00 \mathrm{E}+00$ & $0.00 \mathrm{E}+00$ & $0.00 \mathrm{E}+00$ & $0.00 \mathrm{E}+00$ & $0.00 \mathrm{E}+00$ & $1.37 \mathrm{E}-01$ \\
\hline $85 \%$ & $1.25 \mathrm{E}-02$ & $4.08 \mathrm{E}-03$ & $1.38 \mathrm{E}-01$ & 7.44E-04 & $6.79 \mathrm{E}-05$ & $1.25 \mathrm{E}-02$ & $1.95 \mathrm{E}-07$ & $2.20 \mathrm{E}-09$ & 7.47E-09 & $1.23 \mathrm{E}-09$ & $3.58 \mathrm{E}-11$ & $1.61 \mathrm{E}-01$ \\
\hline $90 \%$ & $1.40 \mathrm{E}-02$ & $4.73 \mathrm{E}-03$ & $1.72 \mathrm{E}-01$ & 8.04E-04 & $8.06 \mathrm{E}-05$ & $1.34 \mathrm{E}-02$ & $5.21 \mathrm{E}-06$ & $6.92 \mathrm{E}-08$ & $2.01 \mathrm{E}-07$ & $3.60 \mathrm{E}-08$ & $9.94 \mathrm{E}-10$ & $1.96 \mathrm{E}-01$ \\
\hline $95 \%$ & $1.64 \mathrm{E}-02$ & $5.87 \mathrm{E}-03$ & $2.37 \mathrm{E}-01$ & $8.98 \mathrm{E}-04$ & $1.05 \mathrm{E}-04$ & $1.46 \mathrm{E}-02$ & $6.35 \mathrm{E}-05$ & $7.90 \mathrm{E}-07$ & $2.46 \mathrm{E}-06$ & $4.25 \mathrm{E}-07$ & $1.04 \mathrm{E}-08$ & $2.68 \mathrm{E}-01$ \\
\hline Mean & 8.83E-03 & $2.57 \mathrm{E}-03$ & 8.04E-02 & $5.95 \mathrm{E}-04$ & $4.16 \mathrm{E}-05$ & $8.54 \mathrm{E}-03$ & $4.51 \mathrm{E}-03$ & $1.13 \mathrm{E}-04$ & $1.25 \mathrm{E}-04$ & $1.48 \mathrm{E}-06$ & $1.55 \mathrm{E}-07$ & $1.05 \mathrm{E}-01$ \\
\hline Min & $1.40 \mathrm{E}-11$ & $5.49 \mathrm{E}-12$ & $2.30 \mathrm{E}-10$ & $2.22 \mathrm{E}-12$ & $1.35 \mathrm{E}-13$ & $2.85 \mathrm{E}-11$ & $0.00 \mathrm{E}+00$ & $0.00 \mathrm{E}+00$ & $0.00 \mathrm{E}+00$ & $0.00 \mathrm{E}+00$ & $0.00 \mathrm{E}+00$ & $2.81 \mathrm{E}-10$ \\
\hline Max & $2.37 \mathrm{E}-02$ & $1.96 \mathrm{E}-02$ & $2.19 \mathrm{E}+00$ & $2.34 \mathrm{E}-03$ & 4.19E-04 & $1.97 \mathrm{E}-02$ & $4.68 \mathrm{E}+00$ & $1.18 \mathrm{E}-01$ & $9.48 \mathrm{E}-02$ & $1.08 \mathrm{E}-03$ & $1.04 \mathrm{E}-04$ & $4.89 \mathrm{E}+00$ \\
\hline
\end{tabular}


TABLE C.26 Peak DSR Percentiles (mrem/yr per pCi/g) for Different Exposure Pathways for Ba-133 in Onsite Resident Scenario

\begin{tabular}{|c|c|c|c|c|c|c|c|c|c|c|c|c|}
\hline Percentile & External & $\begin{array}{c}\text { Inhalation } \\
\text { w/o Rn }\end{array}$ & $\begin{array}{c}\text { Plant } \\
\text { Ingestion } \\
(\mathrm{WI})\end{array}$ & $\begin{array}{c}\text { Meat } \\
\text { Ingestion } \\
(\mathrm{WI})\end{array}$ & $\begin{array}{c}\text { Milk } \\
\text { Ingestion } \\
(\mathrm{WI})\end{array}$ & $\begin{array}{c}\text { Soil } \\
\text { Ingestion }\end{array}$ & $\begin{array}{c}\text { Water } \\
\text { Ingestion }\end{array}$ & $\begin{array}{l}\text { Aquatic } \\
\text { Foods }\end{array}$ & $\begin{array}{c}\text { Plant } \\
\text { Ingestion } \\
\text { (WD) }\end{array}$ & $\begin{array}{c}\text { Meat } \\
\text { Ingestion } \\
\text { (WD) }\end{array}$ & $\begin{array}{c}\text { Milk } \\
\text { Ingestion } \\
\text { (WD) }\end{array}$ & $\begin{array}{c}\text { All } \\
\text { Pathways }\end{array}$ \\
\hline $5 \%$ & $.72 \mathrm{E}-06$ & $8.51 \mathrm{E}-13$ & $1.52 \mathrm{E}-08$ & $1.46 \mathrm{E}-10$ & $4.88 \mathrm{E}-10$ & $2.74 \mathrm{E}-10$ & $0.00 \mathrm{E}+00$ & $0.00 \mathrm{E}+00$ & $0.00 \mathrm{E}+00$ & $0.00 \mathrm{E}+00$ & $0.00 \mathrm{E}+00$ & $1.95 \mathrm{E}-06$ \\
\hline $10 \%$ & $9.61 \mathrm{E}-05$ & $4.34 \mathrm{E}-11$ & $4.90 \mathrm{E}-07$ & $5.21 \mathrm{E}-09$ & $2.25 \mathrm{E}-08$ & $1.21 \mathrm{E}-08$ & $0.00 \mathrm{E}+00$ & $0.00 \mathrm{E}+00$ & $0.00 \mathrm{E}+00$ & $0.00 \mathrm{E}+00$ & $0.00 \mathrm{E}+00$ & $9.88 \mathrm{E}-05$ \\
\hline $15 \%$ & $2.63 \mathrm{E}-04$ & $9.94 \mathrm{E}-11$ & $1.05 \mathrm{E}-06$ & $1.02 \mathrm{E}-08$ & $4.50 \mathrm{E}-08$ & $2.63 \mathrm{E}-08$ & $0.00 \mathrm{E}+00$ & $0.00 \mathrm{E}+00$ & $0.00 \mathrm{E}+00$ & $0.00 \mathrm{E}+00$ & $0.00 \mathrm{E}+00$ & $2.71 \mathrm{E}-04$ \\
\hline $20 \%$ & $3.61 \mathrm{E}-04$ & $1.45 \mathrm{E}-10$ & $1.56 \mathrm{E}-06$ & $1.52 \mathrm{E}-08$ & $6.20 \mathrm{E}-08$ & $3.90 \mathrm{E}-08$ & $0.00 \mathrm{E}+00$ & $0.00 \mathrm{E}+00$ & $0.00 \mathrm{E}+00$ & $0.00 \mathrm{E}+00$ & $0.00 \mathrm{E}+00$ & $3.68 \mathrm{E}-04$ \\
\hline $30 \%$ & $4.46 \mathrm{E}-04$ & $2.05 \mathrm{E}-10$ & $2.64 \mathrm{E}-06$ & $2.53 \mathrm{E}-08$ & $9.47 \mathrm{E}-08$ & $6.11 \mathrm{E}-08$ & $0.00 \mathrm{E}+00$ & $0.00 \mathrm{E}+00$ & $0.00 \mathrm{E}+00$ & $0.00 \mathrm{E}+00$ & $0.00 \mathrm{E}+00$ & $4.60 \mathrm{E}-04$ \\
\hline $35 \%$ & $4.82 \mathrm{E}-04$ & $2.35 \mathrm{E}-10$ & $3.21 \mathrm{E}-06$ & $3.08 \mathrm{E}-08$ & $1.11 \mathrm{E}-07$ & $7.10 \mathrm{E}-08$ & $0.00 \mathrm{E}+00$ & $0.00 \mathrm{E}+00$ & $0.00 \mathrm{E}+00$ & $0.00 \mathrm{E}+00$ & $0.00 \mathrm{E}+00$ & $4.92 \mathrm{E}-04$ \\
\hline $40 \%$ & $5.15 \mathrm{E}-04$ & $2.69 \mathrm{E}-10$ & $3.90 \mathrm{E}-06$ & $3.59 \mathrm{E}-08$ & $1.28 \mathrm{E}-07$ & $8.14 \mathrm{E}-08$ & $0.00 \mathrm{E}+00$ & $0.00 \mathrm{E}+00$ & $0.00 \mathrm{E}+00$ & $0.00 \mathrm{E}+00$ & $0.00 \mathrm{E}+00$ & $5.27 \mathrm{E}-04$ \\
\hline $45 \%$ & $5.53 \mathrm{E}-04$ & $3.02 \mathrm{E}-10$ & $4.59 \mathrm{E}-06$ & $4.36 \mathrm{E}-08$ & $1.47 \mathrm{E}-07$ & $8.88 \mathrm{E}-08$ & $0.00 \mathrm{E}+00$ & $0.00 \mathrm{E}+00$ & $0.00 \mathrm{E}+00$ & $0.00 \mathrm{E}+00$ & $0.00 \mathrm{E}+00$ & $5.65 \mathrm{E}-04$ \\
\hline $50 \%$ & $5.92 \mathrm{E}-04$ & $3.32 \mathrm{E}-10$ & $5.42 \mathrm{E}-06$ & $5.20 \mathrm{E}-08$ & $1.67 \mathrm{E}-07$ & $9.73 \mathrm{E}-08$ & $0.00 \mathrm{E}+00$ & $0.00 \mathrm{E}+00$ & $0.00 \mathrm{E}+00$ & $0.00 \mathrm{E}+00$ & $0.00 \mathrm{E}+00$ & $6.05 \mathrm{E}-04$ \\
\hline $55 \%$ & $6.28 \mathrm{E}-04$ & $3.66 \mathrm{E}-10$ & $6.30 \mathrm{E}-06$ & $6.11 \mathrm{E}-08$ & $1.91 \mathrm{E}-07$ & $1.05 \mathrm{E}-07$ & $0.00 \mathrm{E}+00$ & $0.00 \mathrm{E}+00$ & $0.00 \mathrm{E}+00$ & $0.00 \mathrm{E}+00$ & $0.00 \mathrm{E}+00$ & $6.43 \mathrm{E}-04$ \\
\hline $60 \%$ & $6.70 \mathrm{E}-04$ & $4.01 \mathrm{E}-10$ & 7.43E-06 & $7.01 \mathrm{E}-08$ & $2.22 \mathrm{E}-07$ & $1.12 \mathrm{E}-07$ & $0.00 \mathrm{E}+00$ & $0.00 \mathrm{E}+00$ & $0.00 \mathrm{E}+00$ & $0.00 \mathrm{E}+00$ & $0.00 \mathrm{E}+00$ & $6.79 \mathrm{E}-04$ \\
\hline $65 \%$ & $7.06 \mathrm{E}-04$ & $4.38 \mathrm{E}-10$ & $8.59 \mathrm{E}-06$ & $8.32 \mathrm{E}-08$ & $2.51 \mathrm{E}-07$ & $1.19 \mathrm{E}-07$ & $0.00 \mathrm{E}+00$ & $0.00 \mathrm{E}+00$ & $0.00 \mathrm{E}+00$ & $0.00 \mathrm{E}+00$ & $0.00 \mathrm{E}+00$ & $7.21 \mathrm{E}-04$ \\
\hline $80 \%$ & $8.88 \mathrm{E}-04$ & $5.94 \mathrm{E}-10$ & $1.48 \mathrm{E}-05$ & $1.46 \mathrm{E}-07$ & $4.04 \mathrm{E}-07$ & $1.45 \mathrm{E}-07$ & $0.00 \mathrm{E}+00$ & $0.00 \mathrm{E}+00$ & $0.00 \mathrm{E}+00$ & $0.00 \mathrm{E}+00$ & $0.00 \mathrm{E}+00$ & $9.02 \mathrm{E}-04$ \\
\hline $85 \%$ & $9.78 \mathrm{E}-04$ & $6.69 \mathrm{E}-10$ & $1.84 \mathrm{E}-05$ & $1.84 \mathrm{E}-07$ & $4.89 \mathrm{E}-07$ & $1.56 \mathrm{E}-07$ & $0.00 \mathrm{E}+00$ & $0.00 \mathrm{E}+00$ & $0.00 \mathrm{E}+00$ & $0.00 \mathrm{E}+00$ & $0.00 \mathrm{E}+00$ & $9.97 \mathrm{E}-04$ \\
\hline $90 \%$ & $1.10 \mathrm{E}-03$ & $7.99 \mathrm{E}-10$ & $2.44 \mathrm{E}-05$ & $2.49 \mathrm{E}-07$ & $6.34 \mathrm{E}-07$ & $1.69 \mathrm{E}-07$ & $0.00 \mathrm{E}+00$ & $0.00 \mathrm{E}+00$ & $0.00 \mathrm{E}+00$ & $0.00 \mathrm{E}+00$ & $0.00 \mathrm{E}+00$ & $1.11 \mathrm{E}-03$ \\
\hline $95 \%$ & $1.29 \mathrm{E}-03$ & $9.99 \mathrm{E}-10$ & $3.83 \mathrm{E}-05$ & $3.96 \mathrm{E}-07$ & $9.55 \mathrm{E}-07$ & $1.85 \mathrm{E}-07$ & $0.00 \mathrm{E}+00$ & $0.00 \mathrm{E}+00$ & $0.00 \mathrm{E}+00$ & $0.00 \mathrm{E}+00$ & $0.00 \mathrm{E}+00$ & $1.31 \mathrm{E}-03$ \\
\hline Mean & $6.21 \mathrm{E}-04$ & $3.94 \mathrm{E}-10$ & $1.08 \mathrm{E}-05$ & $1.10 \mathrm{E}-07$ & $3.00 \mathrm{E}-07$ & $9.45 \mathrm{E}-08$ & $5.76 \mathrm{E}-08$ & $2.98 \mathrm{E}-10$ & $2.22 \mathrm{E}-09$ & $2.03 \mathrm{E}-10$ & $8.31 \mathrm{E}-10$ & $6.32 \mathrm{E}-04$ \\
\hline Min & $0.00 \mathrm{E}+00$ & $0.00 \mathrm{E}+00$ & $0.00 \mathrm{E}+00$ & $0.00 \mathrm{E}+00$ & $0.00 \mathrm{E}+00$ & $0.00 \mathrm{E}+00$ & $0.00 \mathrm{E}+00$ & $0.00 \mathrm{E}+00$ & $0.00 \mathrm{E}+00$ & $0.00 \mathrm{E}+00$ & $0.00 \mathrm{E}+00$ & $0.00 \mathrm{E}+00$ \\
\hline Max & $2.00 \mathrm{E}-03$ & $3.58 \mathrm{E}-09$ & $5.15 \mathrm{E}-04$ & $8.98 \mathrm{E}-06$ & $1.36 \mathrm{E}-05$ & $2.32 \mathrm{E}-07$ & $5.56 \mathrm{E}-05$ & $3.98 \mathrm{E}-07$ & $2.15 \mathrm{E}-06$ & $3.51 \mathrm{E}-07$ & $1.05 \mathrm{E}-06$ & $2.04 \mathrm{E}-03$ \\
\hline
\end{tabular}




\section{TABLE C.27 Peak DSR Percentiles (mrem/yr per pCi/g) for Different Exposure Pathways for C-14 in Onsite Resident Scenario}

\begin{tabular}{|c|c|c|c|c|c|c|c|c|c|c|c|c|}
\hline Percentile & External & $\begin{array}{c}\text { Inhalation } \\
\text { w/o Rn }\end{array}$ & $\begin{array}{c}\text { Plant } \\
\text { Ingestion } \\
\text { (WI) }\end{array}$ & $\begin{array}{c}\text { Meat } \\
\text { Ingestion } \\
(\mathrm{WI})\end{array}$ & $\begin{array}{c}\text { Milk } \\
\text { Ingestion } \\
\text { (WI) }\end{array}$ & $\begin{array}{c}\text { Soil } \\
\text { Ingestion }\end{array}$ & $\begin{array}{c}\text { Water } \\
\text { Ingestion }\end{array}$ & $\begin{array}{l}\text { Aquatic } \\
\text { Foods }\end{array}$ & $\begin{array}{c}\text { Plant } \\
\text { Ingestion } \\
\text { (WD) }\end{array}$ & $\begin{array}{c}\text { Meat } \\
\text { Ingestion } \\
\text { (WD) }\end{array}$ & $\begin{array}{c}\text { Milk } \\
\text { Ingestion } \\
\text { (WD) }\end{array}$ & $\begin{array}{c}\text { All } \\
\text { Pathways }\end{array}$ \\
\hline $5 \%$ & $0.00 \mathrm{E}+00$ & $0.00 \mathrm{E}+00$ & $0.00 \mathrm{E}+00$ & $0.00 \mathrm{E}+00$ & $0.00 \mathrm{E}+00$ & $0.00 \mathrm{E}+00$ & $0.00 \mathrm{E}+00$ & $0.00 \mathrm{E}+00$ & $0.00 \mathrm{E}+00$ & $0.00 \mathrm{E}+00$ & $0.00 \mathrm{E}+00$ & $0.00 \mathrm{E}+00$ \\
\hline $10 \%$ & $0.00 \mathrm{E}+00$ & $0.00 \mathrm{E}+00$ & $0.00 \mathrm{E}+00$ & $0.00 \mathrm{E}+00$ & $0.00 \mathrm{E}+00$ & $0.00 \mathrm{E}+00$ & $0.00 \mathrm{E}+00$ & $0.00 \mathrm{E}+00$ & $0.00 \mathrm{E}+00$ & $0.00 \mathrm{E}+00$ & $0.00 \mathrm{E}+00$ & $0.00 \mathrm{E}+00$ \\
\hline $15 \%$ & $0.00 \mathrm{E}+00$ & $0.00 \mathrm{E}+00$ & $0.00 \mathrm{E}+00$ & $0.00 \mathrm{E}+00$ & $0.00 \mathrm{E}+00$ & $0.00 \mathrm{E}+00$ & $0.00 \mathrm{E}+00$ & $0.00 \mathrm{E}+00$ & $0.00 \mathrm{E}+00$ & $0.00 \mathrm{E}+00$ & $0.00 \mathrm{E}+00$ & $0.00 \mathrm{E}+00$ \\
\hline $20 \%$ & $0.00 \mathrm{E}+00$ & $0.00 \mathrm{E}+00$ & $0.00 \mathrm{E}+00$ & $0.00 \mathrm{E}+00$ & $0.00 \mathrm{E}+00$ & $0.00 \mathrm{E}+00$ & $0.00 \mathrm{E}+00$ & $0.00 \mathrm{E}+00$ & $0.00 \mathrm{E}+00$ & $0.00 \mathrm{E}+00$ & $0.00 \mathrm{E}+00$ & $0.00 \mathrm{E}+00$ \\
\hline $30 \%$ & $0.00 \mathrm{E}+00$ & $0.00 \mathrm{E}+00$ & $0.00 \mathrm{E}+00$ & $0.00 \mathrm{E}+00$ & $0.00 \mathrm{E}+00$ & $0.00 \mathrm{E}+00$ & $0.00 \mathrm{E}+00$ & $0.00 \mathrm{E}+00$ & $0.00 \mathrm{E}+00$ & $0.00 \mathrm{E}+00$ & $0.00 \mathrm{E}+00$ & $0.00 \mathrm{E}+00$ \\
\hline $35 \%$ & $0.00 \mathrm{E}+00$ & $0.00 \mathrm{E}+00$ & $0.00 \mathrm{E}+00$ & $0.00 \mathrm{E}+00$ & $0.00 \mathrm{E}+00$ & $0.00 \mathrm{E}+00$ & $0.00 \mathrm{E}+00$ & $0.00 \mathrm{E}+00$ & $0.00 \mathrm{E}+00$ & $0.00 \mathrm{E}+00$ & $0.00 \mathrm{E}+00$ & $0.00 \mathrm{E}+00$ \\
\hline $40 \%$ & $0.00 \mathrm{E}+00$ & $0.00 \mathrm{E}+00$ & $0.00 \mathrm{E}+00$ & $0.00 \mathrm{E}+00$ & $0.00 \mathrm{E}+00$ & $0.00 \mathrm{E}+00$ & $0.00 \mathrm{E}+00$ & $0.00 \mathrm{E}+00$ & $0.00 \mathrm{E}+00$ & $0.00 \mathrm{E}+00$ & $0.00 \mathrm{E}+00$ & $0.00 \mathrm{E}+00$ \\
\hline $45 \%$ & $0.00 \mathrm{E}+00$ & $0.00 \mathrm{E}+00$ & $0.00 \mathrm{E}+00$ & $0.00 \mathrm{E}+00$ & $0.00 \mathrm{E}+00$ & $0.00 \mathrm{E}+00$ & $0.00 \mathrm{E}+00$ & $0.00 \mathrm{E}+00$ & $0.00 \mathrm{E}+00$ & $0.00 \mathrm{E}+00$ & $0.00 \mathrm{E}+00$ & $0.00 \mathrm{E}+00$ \\
\hline $50 \%$ & $0.00 \mathrm{E}+00$ & $0.00 \mathrm{E}+00$ & $0.00 \mathrm{E}+00$ & $0.00 \mathrm{E}+00$ & $0.00 \mathrm{E}+00$ & $0.00 \mathrm{E}+00$ & $0.00 \mathrm{E}+00$ & $0.00 \mathrm{E}+00$ & $0.00 \mathrm{E}+00$ & $0.00 \mathrm{E}+00$ & $0.00 \mathrm{E}+00$ & $0.00 \mathrm{E}+00$ \\
\hline $55 \%$ & $0.00 \mathrm{E}+00$ & $0.00 \mathrm{E}+00$ & $0.00 \mathrm{E}+00$ & $0.00 \mathrm{E}+00$ & $0.00 \mathrm{E}+00$ & $0.00 \mathrm{E}+00$ & $0.00 \mathrm{E}+00$ & $0.00 \mathrm{E}+00$ & $0.00 \mathrm{E}+00$ & $0.00 \mathrm{E}+00$ & $0.00 \mathrm{E}+00$ & $0.00 \mathrm{E}+00$ \\
\hline $60 \%$ & $0.00 \mathrm{E}+00$ & $0.00 \mathrm{E}+00$ & $0.00 \mathrm{E}+00$ & $0.00 \mathrm{E}+00$ & $0.00 \mathrm{E}+00$ & $0.00 \mathrm{E}+00$ & $0.00 \mathrm{E}+00$ & $0.00 \mathrm{E}+00$ & $0.00 \mathrm{E}+00$ & $0.00 \mathrm{E}+00$ & $0.00 \mathrm{E}+00$ & $0.00 \mathrm{E}+00$ \\
\hline $65 \%$ & $0.00 \mathrm{E}+00$ & $0.00 \mathrm{E}+00$ & $0.00 \mathrm{E}+00$ & $0.00 \mathrm{E}+00$ & $0.00 \mathrm{E}+00$ & $0.00 \mathrm{E}+00$ & $0.00 \mathrm{E}+00$ & $0.00 \mathrm{E}+00$ & $0.00 \mathrm{E}+00$ & $0.00 \mathrm{E}+00$ & $0.00 \mathrm{E}+00$ & $0.00 \mathrm{E}+00$ \\
\hline $80 \%$ & $0.00 \mathrm{E}+00$ & $0.00 \mathrm{E}+00$ & $0.00 \mathrm{E}+00$ & $0.00 \mathrm{E}+00$ & $0.00 \mathrm{E}+00$ & $0.00 \mathrm{E}+00$ & $0.00 \mathrm{E}+00$ & $0.00 \mathrm{E}+00$ & $0.00 \mathrm{E}+00$ & $0.00 \mathrm{E}+00$ & $0.00 \mathrm{E}+00$ & $0.00 \mathrm{E}+00$ \\
\hline $85 \%$ & $0.00 \mathrm{E}+00$ & $0.00 \mathrm{E}+00$ & $0.00 \mathrm{E}+00$ & $0.00 \mathrm{E}+00$ & $0.00 \mathrm{E}+00$ & $0.00 \mathrm{E}+00$ & $1.77 \mathrm{E}-04$ & $5.95 \mathrm{E}-04$ & $1.94 \mathrm{E}-05$ & $8.11 \mathrm{E}-06$ & $2.26 \mathrm{E}-05$ & $1.01 \mathrm{E}-03$ \\
\hline $90 \%$ & $0.00 \mathrm{E}+00$ & $0.00 \mathrm{E}+00$ & $0.00 \mathrm{E}+00$ & $0.00 \mathrm{E}+00$ & $0.00 \mathrm{E}+00$ & $0.00 \mathrm{E}+00$ & $5.70 \mathrm{E}-04$ & $2.46 \mathrm{E}-03$ & $6.22 \mathrm{E}-05$ & $2.52 \mathrm{E}-05$ & $7.21 \mathrm{E}-05$ & $3.45 \mathrm{E}-03$ \\
\hline $95 \%$ & $0.00 \mathrm{E}+00$ & $0.00 \mathrm{E}+00$ & $0.00 \mathrm{E}+00$ & $0.00 \mathrm{E}+00$ & $0.00 \mathrm{E}+00$ & $0.00 \mathrm{E}+00$ & $1.21 \mathrm{E}-03$ & $7.98 \mathrm{E}-03$ & $1.35 \mathrm{E}-04$ & $5.33 \mathrm{E}-05$ & $1.51 \mathrm{E}-04$ & $1.05 \mathrm{E}-02$ \\
\hline Mean & $7.48 \mathrm{E}-10$ & $5.07 \mathrm{E}-08$ & $3.01 \mathrm{E}-04$ & $1.17 \mathrm{E}-04$ & $4.00 \mathrm{E}-05$ & $5.17 \mathrm{E}-09$ & $1.91 \mathrm{E}-04$ & $3.11 \mathrm{E}-03$ & $2.19 \mathrm{E}-05$ & $8.52 \mathrm{E}-06$ & $2.40 \mathrm{E}-05$ & $3.36 \mathrm{E}-03$ \\
\hline Min & $0.00 \mathrm{E}+00$ & $0.00 \mathrm{E}+00$ & $0.00 \mathrm{E}+00$ & $0.00 \mathrm{E}+00$ & $0.00 \mathrm{E}+00$ & $0.00 \mathrm{E}+00$ & $0.00 \mathrm{E}+00$ & $0.00 \mathrm{E}+00$ & $0.00 \mathrm{E}+00$ & $0.00 \mathrm{E}+00$ & $0.00 \mathrm{E}+00$ & $0.00 \mathrm{E}+00$ \\
\hline Max & $1.02 \mathrm{E}-06$ & $5.13 \mathrm{E}-05$ & $3.02 \mathrm{E}-01$ & $1.17 \mathrm{E}-01$ & 4.01E-02 & 7.72E-06 & $1.88 \mathrm{E}-02$ & $3.00 \mathrm{E}+00$ & $1.78 \mathrm{E}-03$ & 7.99E-04 & $2.35 \mathrm{E}-03$ & $3.00 \mathrm{E}+00$ \\
\hline
\end{tabular}


TABLE C.28 Peak DSR Percentiles (mrem/yr per pCi/g) for Different Exposure Pathways for Cs-137 in Onsite Resident Scenario

\begin{tabular}{|c|c|c|c|c|c|c|c|c|c|c|c|c|}
\hline Percentile & External & $\begin{array}{c}\text { Inhalation } \\
\text { w/o Rn }\end{array}$ & $\begin{array}{c}\text { Plant } \\
\text { Ingestion } \\
(\mathrm{WI})\end{array}$ & $\begin{array}{c}\text { Meat } \\
\text { Ingestion } \\
\text { (WI) }\end{array}$ & $\begin{array}{c}\text { Milk } \\
\text { Ingestion } \\
(\mathrm{WI})\end{array}$ & $\begin{array}{c}\text { Soil } \\
\text { Ingestion }\end{array}$ & $\begin{array}{c}\text { Water } \\
\text { Ingestion }\end{array}$ & $\begin{array}{c}\text { Aquatic } \\
\text { Foods }\end{array}$ & $\begin{array}{c}\text { Plant } \\
\text { Ingestion } \\
\text { (WD) }\end{array}$ & $\begin{array}{c}\text { Meat } \\
\text { Ingestion } \\
\text { (WD) }\end{array}$ & $\begin{array}{c}\text { Milk } \\
\text { Ingestion } \\
\text { (WD) }\end{array}$ & $\begin{array}{c}\text { All } \\
\text { Pathways }\end{array}$ \\
\hline $5 \%$ & $4.31 \mathrm{E}-02$ & $3.22 \mathrm{E}-08$ & $3.03 \mathrm{E}-03$ & $6.08 \mathrm{E}-03$ & $1.47 \mathrm{E}-03$ & $2.00 \mathrm{E}-05$ & $0.00 \mathrm{E}+00$ & $0.00 \mathrm{E}+00$ & $0.00 \mathrm{E}+00$ & $0.00 \mathrm{E}+00$ & $0.00 \mathrm{E}+00$ & 7.02E-02 \\
\hline $10 \%$ & $4.80 \mathrm{E}-02$ & $4.36 \mathrm{E}-08$ & $4.37 \mathrm{E}-03$ & 7.97E-03 & $1.93 \mathrm{E}-03$ & $2.81 \mathrm{E}-05$ & $0.00 \mathrm{E}+00$ & $0.00 \mathrm{E}+00$ & $0.00 \mathrm{E}+00$ & $0.00 \mathrm{E}+00$ & $0.00 \mathrm{E}+00$ & 7.93E-02 \\
\hline $15 \%$ & $5.20 \mathrm{E}-02$ & $5.04 \mathrm{E}-08$ & $5.60 \mathrm{E}-03$ & $9.57 \mathrm{E}-03$ & $2.37 \mathrm{E}-03$ & $3.44 \mathrm{E}-05$ & $0.00 \mathrm{E}+00$ & $0.00 \mathrm{E}+00$ & $0.00 \mathrm{E}+00$ & $0.00 \mathrm{E}+00$ & $0.00 \mathrm{E}+00$ & $8.78 \mathrm{E}-02$ \\
\hline $20 \%$ & $5.55 \mathrm{E}-02$ & $5.70 \mathrm{E}-08$ & $6.83 \mathrm{E}-03$ & $1.12 \mathrm{E}-02$ & $2.76 \mathrm{E}-03$ & $3.98 \mathrm{E}-05$ & $0.00 \mathrm{E}+00$ & $0.00 \mathrm{E}+00$ & $0.00 \mathrm{E}+00$ & $0.00 \mathrm{E}+00$ & $0.00 \mathrm{E}+00$ & $9.41 \mathrm{E}-02$ \\
\hline $30 \%$ & $6.23 \mathrm{E}-02$ & $7.29 \mathrm{E}-08$ & $9.35 \mathrm{E}-03$ & $1.45 \mathrm{E}-02$ & $3.61 \mathrm{E}-03$ & $4.89 \mathrm{E}-05$ & $0.00 \mathrm{E}+00$ & $0.00 \mathrm{E}+00$ & $0.00 \mathrm{E}+00$ & $0.00 \mathrm{E}+00$ & $0.00 \mathrm{E}+00$ & $1.07 \mathrm{E}-01$ \\
\hline $35 \%$ & $6.55 \mathrm{E}-02$ & $8.04 \mathrm{E}-08$ & $1.07 \mathrm{E}-02$ & $1.65 \mathrm{E}-02$ & 4.07E-03 & 5.31E-05 & $0.00 \mathrm{E}+00$ & $0.00 \mathrm{E}+00$ & $0.00 \mathrm{E}+00$ & $0.00 \mathrm{E}+00$ & $0.00 \mathrm{E}+00$ & $1.14 \mathrm{E}-01$ \\
\hline $40 \%$ & $6.89 \mathrm{E}-02$ & 8.63E-08 & $1.22 \mathrm{E}-02$ & $1.84 \mathrm{E}-02$ & $4.60 \mathrm{E}-03$ & 5.67E-05 & $0.00 \mathrm{E}+00$ & $0.00 \mathrm{E}+00$ & $0.00 \mathrm{E}+00$ & $0.00 \mathrm{E}+00$ & $0.00 \mathrm{E}+00$ & $1.21 \mathrm{E}-01$ \\
\hline $45 \%$ & 7.22E-02 & $9.40 \mathrm{E}-08$ & $1.40 \mathrm{E}-02$ & 2.07E-02 & $5.12 \mathrm{E}-03$ & $6.05 \mathrm{E}-05$ & $0.00 \mathrm{E}+00$ & $0.00 \mathrm{E}+00$ & $0.00 \mathrm{E}+00$ & $0.00 \mathrm{E}+00$ & $0.00 \mathrm{E}+00$ & $1.28 \mathrm{E}-01$ \\
\hline $50 \%$ & 7.64E-02 & $1.02 \mathrm{E}-07$ & $1.58 \mathrm{E}-02$ & 2.33E-02 & $5.79 \mathrm{E}-03$ & $6.37 \mathrm{E}-05$ & $0.00 \mathrm{E}+00$ & $0.00 \mathrm{E}+00$ & $0.00 \mathrm{E}+00$ & $0.00 \mathrm{E}+00$ & $0.00 \mathrm{E}+00$ & $1.36 \mathrm{E}-01$ \\
\hline $55 \%$ & 8.02E-02 & $1.10 \mathrm{E}-07$ & $1.79 \mathrm{E}-02$ & $2.62 \mathrm{E}-02$ & $6.49 \mathrm{E}-03$ & $6.73 \mathrm{E}-05$ & $0.00 \mathrm{E}+00$ & $0.00 \mathrm{E}+00$ & $0.00 \mathrm{E}+00$ & $0.00 \mathrm{E}+00$ & $0.00 \mathrm{E}+00$ & $1.43 \mathrm{E}-01$ \\
\hline $60 \%$ & $8.46 \mathrm{E}-02$ & $1.19 \mathrm{E}-07$ & $2.03 \mathrm{E}-02$ & 2.94E-02 & 7.34E-03 & $7.06 \mathrm{E}-05$ & $0.00 \mathrm{E}+00$ & $0.00 \mathrm{E}+00$ & $0.00 \mathrm{E}+00$ & $0.00 \mathrm{E}+00$ & $0.00 \mathrm{E}+00$ & $1.52 \mathrm{E}-01$ \\
\hline $65 \%$ & $8.95 \mathrm{E}-02$ & $1.29 \mathrm{E}-07$ & 2.32E-02 & 3.39E-02 & 8.31E-03 & $7.47 \mathrm{E}-05$ & $0.00 \mathrm{E}+00$ & $0.00 \mathrm{E}+00$ & $0.00 \mathrm{E}+00$ & $0.00 \mathrm{E}+00$ & $0.00 \mathrm{E}+00$ & $1.61 \mathrm{E}-01$ \\
\hline $75 \%$ & $1.01 \mathrm{E}-01$ & $1.51 \mathrm{E}-07$ & $3.11 \mathrm{E}-02$ & $4.40 \mathrm{E}-02$ & $1.09 \mathrm{E}-02$ & $8.29 \mathrm{E}-05$ & $0.00 \mathrm{E}+00$ & $0.00 \mathrm{E}+00$ & $0.00 \mathrm{E}+00$ & $0.00 \mathrm{E}+00$ & $0.00 \mathrm{E}+00$ & $1.83 \mathrm{E}-01$ \\
\hline $80 \%$ & $1.09 \mathrm{E}-01$ & $1.66 \mathrm{E}-07$ & $3.66 \mathrm{E}-02$ & $5.17 \mathrm{E}-02$ & $1.31 \mathrm{E}-02$ & $8.79 \mathrm{E}-05$ & $0.00 \mathrm{E}+00$ & $0.00 \mathrm{E}+00$ & $0.00 \mathrm{E}+00$ & $0.00 \mathrm{E}+00$ & $0.00 \mathrm{E}+00$ & $1.99 \mathrm{E}-01$ \\
\hline $85 \%$ & $1.19 \mathrm{E}-01$ & $1.89 \mathrm{E}-07$ & $4.45 \mathrm{E}-02$ & $6.23 \mathrm{E}-02$ & $1.58 \mathrm{E}-02$ & $9.33 \mathrm{E}-05$ & $0.00 \mathrm{E}+00$ & $0.00 \mathrm{E}+00$ & $0.00 \mathrm{E}+00$ & $0.00 \mathrm{E}+00$ & $0.00 \mathrm{E}+00$ & $2.23 \mathrm{E}-01$ \\
\hline $90 \%$ & $1.33 \mathrm{E}-01$ & $2.21 \mathrm{E}-07$ & $5.65 \mathrm{E}-02$ & $8.16 \mathrm{E}-02$ & $2.01 \mathrm{E}-02$ & $9.98 \mathrm{E}-05$ & $0.00 \mathrm{E}+00$ & $0.00 \mathrm{E}+00$ & $0.00 \mathrm{E}+00$ & $0.00 \mathrm{E}+00$ & $0.00 \mathrm{E}+00$ & $2.56 \mathrm{E}-01$ \\
\hline $95 \%$ & $1.55 \mathrm{E}-01$ & $2.73 \mathrm{E}-07$ & $8.11 \mathrm{E}-02$ & $1.19 \mathrm{E}-01$ & $2.90 \mathrm{E}-02$ & $1.09 \mathrm{E}-04$ & $0.00 \mathrm{E}+00$ & $0.00 \mathrm{E}+00$ & $0.00 \mathrm{E}+00$ & $0.00 \mathrm{E}+00$ & $0.00 \mathrm{E}+00$ & $3.17 \mathrm{E}-01$ \\
\hline Mean & $8.42 \mathrm{E}-02$ & $1.20 \mathrm{E}-07$ & $2.60 \mathrm{E}-02$ & $3.87 \mathrm{E}-02$ & $9.63 \mathrm{E}-03$ & $6.39 \mathrm{E}-05$ & $0.00 \mathrm{E}+00$ & $0.00 \mathrm{E}+00$ & $0.00 \mathrm{E}+00$ & $0.00 \mathrm{E}+00$ & $0.00 \mathrm{E}+00$ & $1.59 \mathrm{E}-01$ \\
\hline Min & $2.59 \mathrm{E}-03$ & $2.95 \mathrm{E}-10$ & $5.07 \mathrm{E}-04$ & $1.41 \mathrm{E}-03$ & $2.54 \mathrm{E}-04$ & $2.00 \mathrm{E}-06$ & $0.00 \mathrm{E}+00$ & $0.00 \mathrm{E}+00$ & $0.00 \mathrm{E}+00$ & $0.00 \mathrm{E}+00$ & $0.00 \mathrm{E}+00$ & $5.56 \mathrm{E}-03$ \\
\hline Max & $2.23 \mathrm{E}-01$ & $9.13 \mathrm{E}-07$ & 7.17E-01 & $1.21 \mathrm{E}+00$ & $4.52 \mathrm{E}-01$ & $1.29 \mathrm{E}-04$ & $0.00 \mathrm{E}+00$ & $0.00 \mathrm{E}+00$ & $0.00 \mathrm{E}+00$ & $0.00 \mathrm{E}+00$ & $0.00 \mathrm{E}+00$ & $2.45 \mathrm{E}+00$ \\
\hline
\end{tabular}


TABLE C.29 Peak DSR Percentiles (mrem/yr per pCi/g) for Different Exposure Pathways for Pu-238 in Onsite Resident Scenario

\begin{tabular}{|c|c|c|c|c|c|c|c|c|c|c|c|c|c|c|}
\hline Percentile & External & $\begin{array}{c}\text { Inhalation } \\
\text { w/o Rn }\end{array}$ & $\begin{array}{l}\text { Radon } \\
\text { (WI) }\end{array}$ & $\begin{array}{c}\text { Plant } \\
\text { Ingestion } \\
\text { (WI) }\end{array}$ & $\begin{array}{c}\text { Meat } \\
\text { Ingestion } \\
\text { (WI) }\end{array}$ & $\begin{array}{c}\text { Milk } \\
\text { Ingestion } \\
\text { (WI) }\end{array}$ & $\begin{array}{c}\text { Soil } \\
\text { Ingestion }\end{array}$ & $\begin{array}{c}\text { Water } \\
\text { Ingestion }\end{array}$ & $\begin{array}{l}\text { Aquatic } \\
\text { Foods }\end{array}$ & $\begin{array}{l}\text { Radon } \\
\text { (WD) }\end{array}$ & $\begin{array}{c}\text { Plant } \\
\text { Ingestion } \\
\text { (WD) }\end{array}$ & $\begin{array}{c}\text { Meat } \\
\text { Ingestion } \\
\text { (WD) }\end{array}$ & $\begin{array}{c}\text { Milk } \\
\text { Ingestion } \\
\text { (WD) }\end{array}$ & $\begin{array}{c}\text { All } \\
\text { Pathways }\end{array}$ \\
\hline $5 \%$ & 7.67E-06 & 4.19E-04 & $8.08 \mathrm{E}-08$ & $7.18 \mathrm{E}-03$ & 4.69E-04 & $4.78 \mathrm{E}-06$ & $1.61 \mathrm{E}-03$ & $0.00 \mathrm{E}+00$ & $0.00 \mathrm{E}+00$ & $0.00 \mathrm{E}+00$ & $0.00 \mathrm{E}+00$ & $0.00 \mathrm{E}+00$ & $0.00 \mathrm{E}+00$ & $1.42 \mathrm{E}-02$ \\
\hline $10 \%$ & $8.58 \mathrm{E}-06$ & $5.69 \mathrm{E}-04$ & $9.58 \mathrm{E}-08$ & $9.90 \mathrm{E}-03$ & $5.13 \mathrm{E}-04$ & $5.71 \mathrm{E}-06$ & $2.28 \mathrm{E}-03$ & $0.00 \mathrm{E}+00$ & $0.00 \mathrm{E}+00$ & $0.00 \mathrm{E}+00$ & $0.00 \mathrm{E}+00$ & $0.00 \mathrm{E}+00$ & $0.00 \mathrm{E}+00$ & $1.74 \mathrm{E}-02$ \\
\hline $15 \%$ & $9.34 \mathrm{E}-06$ & $6.59 \mathrm{E}-04$ & $1.05 \mathrm{E}-07$ & $1.25 \mathrm{E}-02$ & $5.41 \mathrm{E}-04$ & $6.46 \mathrm{E}-06$ & $2.82 \mathrm{E}-03$ & $0.00 \mathrm{E}+00$ & $0.00 \mathrm{E}+00$ & $0.00 \mathrm{E}+00$ & $0.00 \mathrm{E}+00$ & $0.00 \mathrm{E}+00$ & $0.00 \mathrm{E}+00$ & $2.00 \mathrm{E}-02$ \\
\hline $20 \%$ & $9.94 \mathrm{E}-06$ & $7.47 \mathrm{E}-04$ & $1.11 \mathrm{E}-07$ & $1.49 \mathrm{E}-02$ & $5.68 \mathrm{E}-04$ & $7.05 \mathrm{E}-06$ & $3.29 \mathrm{E}-03$ & $0.00 \mathrm{E}+00$ & $0.00 \mathrm{E}+00$ & $0.00 \mathrm{E}+00$ & $0.00 \mathrm{E}+00$ & $0.00 \mathrm{E}+00$ & $0.00 \mathrm{E}+00$ & $2.22 \mathrm{E}-02$ \\
\hline $25 \%$ & $1.05 \mathrm{E}-05$ & $8.42 \mathrm{E}-04$ & $1.16 \mathrm{E}-07$ & $1.74 \mathrm{E}-02$ & $5.93 \mathrm{E}-04$ & $7.63 \mathrm{E}-06$ & $3.67 \mathrm{E}-03$ & $0.00 \mathrm{E}+00$ & $0.00 \mathrm{E}+00$ & $0.00 \mathrm{E}+00$ & $0.00 \mathrm{E}+00$ & $0.00 \mathrm{E}+00$ & $0.00 \mathrm{E}+00$ & $2.48 \mathrm{E}-02$ \\
\hline $30 \%$ & $1.11 \mathrm{E}-05$ & $9.55 \mathrm{E}-04$ & $1.21 \mathrm{E}-07$ & $2.01 \mathrm{E}-02$ & $6.13 \mathrm{E}-04$ & $8.10 \mathrm{E}-06$ & $4.03 \mathrm{E}-03$ & $0.00 \mathrm{E}+00$ & $0.00 \mathrm{E}+00$ & $0.00 \mathrm{E}+00$ & $0.00 \mathrm{E}+00$ & $0.00 \mathrm{E}+00$ & $0.00 \mathrm{E}+00$ & $2.75 \mathrm{E}-02$ \\
\hline $35 \%$ & $1.18 \mathrm{E}-05$ & $1.04 \mathrm{E}-03$ & $1.27 \mathrm{E}-07$ & $2.28 \mathrm{E}-02$ & $6.33 \mathrm{E}-04$ & $8.62 \mathrm{E}-06$ & $4.37 \mathrm{E}-03$ & $0.00 \mathrm{E}+00$ & $0.00 \mathrm{E}+00$ & $0.00 \mathrm{E}+00$ & $0.00 \mathrm{E}+00$ & $0.00 \mathrm{E}+00$ & $0.00 \mathrm{E}+00$ & $3.03 \mathrm{E}-02$ \\
\hline $40 \%$ & $1.24 \mathrm{E}-05$ & $1.13 \mathrm{E}-03$ & $1.31 \mathrm{E}-07$ & $2.57 \mathrm{E}-02$ & $6.54 \mathrm{E}-04$ & $9.17 \mathrm{E}-06$ & $4.68 \mathrm{E}-03$ & $0.00 \mathrm{E}+00$ & $0.00 \mathrm{E}+00$ & $0.00 \mathrm{E}+00$ & $0.00 \mathrm{E}+00$ & $0.00 \mathrm{E}+00$ & $0.00 \mathrm{E}+00$ & $3.33 \mathrm{E}-02$ \\
\hline $45 \%$ & $1.30 \mathrm{E}-05$ & $1.23 \mathrm{E}-03$ & $1.37 \mathrm{E}-07$ & $2.89 \mathrm{E}-02$ & $6.77 \mathrm{E}-04$ & $9.76 \mathrm{E}-06$ & $4.98 \mathrm{E}-03$ & $0.00 \mathrm{E}+00$ & $0.00 \mathrm{E}+00$ & $0.00 \mathrm{E}+00$ & $0.00 \mathrm{E}+00$ & $0.00 \mathrm{E}+00$ & $0.00 \mathrm{E}+00$ & $3.65 \mathrm{E}-02$ \\
\hline $50 \%$ & $1.36 \mathrm{E}-05$ & $1.33 \mathrm{E}-03$ & $1.41 \mathrm{E}-07$ & $3.25 \mathrm{E}-02$ & $6.97 \mathrm{E}-04$ & $1.03 \mathrm{E}-05$ & $5.26 \mathrm{E}-03$ & $0.00 \mathrm{E}+00$ & $0.00 \mathrm{E}+00$ & $0.00 \mathrm{E}+00$ & $0.00 \mathrm{E}+00$ & $0.00 \mathrm{E}+00$ & $0.00 \mathrm{E}+00$ & $4.05 \mathrm{E}-02$ \\
\hline $55 \%$ & $1.44 \mathrm{E}-05$ & $1.44 \mathrm{E}-03$ & $1.45 \mathrm{E}-07$ & $3.65 \mathrm{E}-02$ & $7.18 \mathrm{E}-04$ & $1.10 \mathrm{E}-05$ & $5.52 \mathrm{E}-03$ & $0.00 \mathrm{E}+00$ & $0.00 \mathrm{E}+00$ & $0.00 \mathrm{E}+00$ & $0.00 \mathrm{E}+00$ & $0.00 \mathrm{E}+00$ & $0.00 \mathrm{E}+00$ & 4.43E-02 \\
\hline $60 \%$ & $1.52 \mathrm{E}-05$ & $1.56 \mathrm{E}-03$ & $1.50 \mathrm{E}-07$ & 4.09E-02 & 7.39E-04 & $1.16 \mathrm{E}-05$ & $5.82 \mathrm{E}-03$ & $0.00 \mathrm{E}+00$ & $0.00 \mathrm{E}+00$ & $0.00 \mathrm{E}+00$ & $0.00 \mathrm{E}+00$ & $0.00 \mathrm{E}+00$ & $0.00 \mathrm{E}+00$ & $4.90 \mathrm{E}-02$ \\
\hline $65 \%$ & $1.60 \mathrm{E}-05$ & $1.69 \mathrm{E}-03$ & $1.56 \mathrm{E}-07$ & $4.60 \mathrm{E}-02$ & $7.65 \mathrm{E}-04$ & $1.24 \mathrm{E}-05$ & $6.12 \mathrm{E}-03$ & $0.00 \mathrm{E}+00$ & $0.00 \mathrm{E}+00$ & $0.00 \mathrm{E}+00$ & $0.00 \mathrm{E}+00$ & $0.00 \mathrm{E}+00$ & $0.00 \mathrm{E}+00$ & $5.36 \mathrm{E}-02$ \\
\hline $70 \%$ & $1.70 \mathrm{E}-05$ & $1.83 \mathrm{E}-03$ & $1.62 \mathrm{E}-07$ & $5.23 \mathrm{E}-02$ & 7.93E-04 & $1.32 \mathrm{E}-05$ & $6.47 \mathrm{E}-03$ & $0.00 \mathrm{E}+00$ & $0.00 \mathrm{E}+00$ & $0.00 \mathrm{E}+00$ & $0.00 \mathrm{E}+00$ & $0.00 \mathrm{E}+00$ & $0.00 \mathrm{E}+00$ & $5.97 \mathrm{E}-02$ \\
\hline $75 \%$ & $1.82 \mathrm{E}-05$ & $1.99 \mathrm{E}-03$ & $1.70 \mathrm{E}-07$ & $6.03 \mathrm{E}-02$ & $8.22 \mathrm{E}-04$ & $1.42 \mathrm{E}-05$ & $6.81 \mathrm{E}-03$ & $0.00 \mathrm{E}+00$ & $0.00 \mathrm{E}+00$ & $0.00 \mathrm{E}+00$ & $0.00 \mathrm{E}+00$ & $0.00 \mathrm{E}+00$ & $0.00 \mathrm{E}+00$ & $6.82 \mathrm{E}-02$ \\
\hline $80 \%$ & $1.96 \mathrm{E}-05$ & $2.19 \mathrm{E}-03$ & $1.77 \mathrm{E}-07$ & $7.03 \mathrm{E}-02$ & 8.63E-04 & $1.55 \mathrm{E}-05$ & $7.21 \mathrm{E}-03$ & $0.00 \mathrm{E}+00$ & $0.00 \mathrm{E}+00$ & $0.00 \mathrm{E}+00$ & $0.00 \mathrm{E}+00$ & $0.00 \mathrm{E}+00$ & $0.00 \mathrm{E}+00$ & $7.85 \mathrm{E}-02$ \\
\hline $85 \%$ & $2.14 \mathrm{E}-05$ & $2.49 \mathrm{E}-03$ & $1.87 \mathrm{E}-07$ & 8.47E-02 & $9.10 \mathrm{E}-04$ & $1.70 \mathrm{E}-05$ & $7.66 \mathrm{E}-03$ & $0.00 \mathrm{E}+00$ & $0.00 \mathrm{E}+00$ & $0.00 \mathrm{E}+00$ & $0.00 \mathrm{E}+00$ & $0.00 \mathrm{E}+00$ & $0.00 \mathrm{E}+00$ & $9.26 \mathrm{E}-02$ \\
\hline $90 \%$ & $2.37 \mathrm{E}-05$ & $2.90 \mathrm{E}-03$ & $1.99 \mathrm{E}-07$ & $1.05 \mathrm{E}-01$ & $9.80 \mathrm{E}-04$ & $1.94 \mathrm{E}-05$ & $8.21 \mathrm{E}-03$ & $0.00 \mathrm{E}+00$ & $0.00 \mathrm{E}+00$ & $0.00 \mathrm{E}+00$ & $0.00 \mathrm{E}+00$ & $0.00 \mathrm{E}+00$ & $0.00 \mathrm{E}+00$ & $1.13 \mathrm{E}-01$ \\
\hline $95 \%$ & $2.76 \mathrm{E}-05$ & $3.60 \mathrm{E}-03$ & $2.21 \mathrm{E}-07$ & $1.48 \mathrm{E}-01$ & $1.08 \mathrm{E}-03$ & $2.31 \mathrm{E}-05$ & $8.92 \mathrm{E}-03$ & $0.00 \mathrm{E}+00$ & $0.00 \mathrm{E}+00$ & $0.00 \mathrm{E}+00$ & $0.00 \mathrm{E}+00$ & $0.00 \mathrm{E}+00$ & $0.00 \mathrm{E}+00$ & $1.56 \mathrm{E}-01$ \\
\hline Mean & $1.51 \mathrm{E}-05$ & $1.58 \mathrm{E}-03$ & $1.44 \mathrm{E}-07$ & $4.95 \mathrm{E}-02$ & $7.28 \mathrm{E}-04$ & $1.17 \mathrm{E}-05$ & $5.25 \mathrm{E}-03$ & $1.36 \mathrm{E}-07$ & $9.78 \mathrm{E}-10$ & $9.88 \mathrm{E}-17$ & $5.22 \mathrm{E}-09$ & $1.12 \mathrm{E}-09$ & $1.41 \mathrm{E}-09$ & $5.71 \mathrm{E}-02$ \\
\hline Min & $1.73 \mathrm{E}-09$ & 4.82E-09 & 4.67E-09 & $1.46 \mathrm{E}-06$ & $8.59 \mathrm{E}-08$ & $3.59 \mathrm{E}-08$ & $3.21 \mathrm{E}-08$ & $0.00 \mathrm{E}+00$ & $0.00 \mathrm{E}+00$ & $0.00 \mathrm{E}+00$ & $0.00 \mathrm{E}+00$ & $0.00 \mathrm{E}+00$ & $0.00 \mathrm{E}+00$ & $1.65 \mathrm{E}-06$ \\
\hline Max & $3.97 \mathrm{E}-05$ & $1.19 \mathrm{E}-02$ & $3.51 \mathrm{E}-07$ & $8.99 \mathrm{E}-01$ & $3.63 \mathrm{E}-03$ & $8.14 \mathrm{E}-05$ & $1.07 \mathrm{E}-02$ & $2.67 \mathrm{E}-04$ & $3.52 \mathrm{E}-06$ & $2.86 \mathrm{E}-13$ & $1.03 \mathrm{E}-05$ & $3.01 \mathrm{E}-06$ & $3.26 \mathrm{E}-06$ & $9.12 \mathrm{E}-01$ \\
\hline
\end{tabular}


TABLE C.30 Peak DSR Percentiles (mrem/yr per pCi/g) for Different Exposure Pathways for Pu-239 in Onsite Resident Scenario

\begin{tabular}{|c|c|c|c|c|c|c|c|c|c|c|c|c|}
\hline Percentile & External & $\begin{array}{c}\text { Inhalation } \\
\text { w/o Rn }\end{array}$ & $\begin{array}{c}\text { Plant } \\
\text { Ingestion } \\
(\mathrm{WI})\end{array}$ & $\begin{array}{c}\text { Meat } \\
\text { Ingestion } \\
(\mathrm{WI})\end{array}$ & $\begin{array}{c}\text { Milk } \\
\text { Ingestion } \\
(\mathrm{WI})\end{array}$ & $\begin{array}{c}\text { Soil } \\
\text { Ingestion }\end{array}$ & $\begin{array}{c}\text { Water } \\
\text { Ingestion }\end{array}$ & $\begin{array}{l}\text { Aquatic } \\
\text { Foods }\end{array}$ & $\begin{array}{c}\text { Plant } \\
\text { Ingestion } \\
\text { (WD) }\end{array}$ & $\begin{array}{c}\text { Meat } \\
\text { Ingestion } \\
\text { (WD) }\end{array}$ & $\begin{array}{c}\text { Milk } \\
\text { Ingestion } \\
\text { (WD) }\end{array}$ & $\begin{array}{c}\text { All } \\
\text { Pathways } \\
\end{array}$ \\
\hline $5 \%$ & $3.69 \mathrm{E}-05$ & $1.00 \mathrm{E}-03$ & $1.68 \mathrm{E}-02$ & $1.12 \mathrm{E}-03$ & $1.05 \mathrm{E}-05$ & $3.86 \mathrm{E}-03$ & $0.00 \mathrm{E}+00$ & $0.00 \mathrm{E}+00$ & $0.00 \mathrm{E}+00$ & $0.00 \mathrm{E}+00$ & $0.00 \mathrm{E}+00$ & 3.39E-02 \\
\hline $10 \%$ & 4.13E-05 & $1.37 \mathrm{E}-03$ & $2.38 \mathrm{E}-02$ & $1.23 \mathrm{E}-03$ & $1.27 \mathrm{E}-05$ & $5.50 \mathrm{E}-03$ & $0.00 \mathrm{E}+00$ & $0.00 \mathrm{E}+00$ & $0.00 \mathrm{E}+00$ & $0.00 \mathrm{E}+00$ & $0.00 \mathrm{E}+00$ & 4.18E-02 \\
\hline $15 \%$ & $4.47 \mathrm{E}-05$ & $1.59 \mathrm{E}-03$ & $3.00 \mathrm{E}-02$ & $1.30 \mathrm{E}-03$ & $1.44 \mathrm{E}-05$ & $6.80 \mathrm{E}-03$ & $0.00 \mathrm{E}+00$ & $0.00 \mathrm{E}+00$ & $0.00 \mathrm{E}+00$ & $0.00 \mathrm{E}+00$ & $0.00 \mathrm{E}+00$ & $4.76 \mathrm{E}-02$ \\
\hline $20 \%$ & 4.78E-05 & $1.80 \mathrm{E}-03$ & $3.57 \mathrm{E}-02$ & $1.36 \mathrm{E}-03$ & $1.58 \mathrm{E}-05$ & 7.91E-03 & $0.00 \mathrm{E}+00$ & $0.00 \mathrm{E}+00$ & $0.00 \mathrm{E}+00$ & $0.00 \mathrm{E}+00$ & $0.00 \mathrm{E}+00$ & 5.33E-02 \\
\hline $25 \%$ & $5.07 \mathrm{E}-05$ & $2.03 \mathrm{E}-03$ & $4.18 \mathrm{E}-02$ & $1.42 \mathrm{E}-03$ & $1.71 \mathrm{E}-05$ & $8.80 \mathrm{E}-03$ & $0.00 \mathrm{E}+00$ & $0.00 \mathrm{E}+00$ & $0.00 \mathrm{E}+00$ & $0.00 \mathrm{E}+00$ & $0.00 \mathrm{E}+00$ & $5.98 \mathrm{E}-02$ \\
\hline $30 \%$ & $5.34 \mathrm{E}-05$ & $2.30 \mathrm{E}-03$ & $4.80 \mathrm{E}-02$ & $1.47 \mathrm{E}-03$ & $1.83 \mathrm{E}-05$ & $9.74 \mathrm{E}-03$ & $0.00 \mathrm{E}+00$ & $0.00 \mathrm{E}+00$ & $0.00 \mathrm{E}+00$ & $0.00 \mathrm{E}+00$ & $0.00 \mathrm{E}+00$ & $6.62 \mathrm{E}-02$ \\
\hline $35 \%$ & $5.65 \mathrm{E}-05$ & $2.51 \mathrm{E}-03$ & $5.44 \mathrm{E}-02$ & $1.52 \mathrm{E}-03$ & $1.96 \mathrm{E}-05$ & $1.05 \mathrm{E}-02$ & $0.00 \mathrm{E}+00$ & $0.00 \mathrm{E}+00$ & $0.00 \mathrm{E}+00$ & $0.00 \mathrm{E}+00$ & $0.00 \mathrm{E}+00$ & $7.26 \mathrm{E}-02$ \\
\hline $40 \%$ & 5.93E-05 & $2.74 \mathrm{E}-03$ & $6.15 \mathrm{E}-02$ & $1.57 \mathrm{E}-03$ & $2.08 \mathrm{E}-05$ & $1.12 \mathrm{E}-02$ & $0.00 \mathrm{E}+00$ & $0.00 \mathrm{E}+00$ & $0.00 \mathrm{E}+00$ & $0.00 \mathrm{E}+00$ & $0.00 \mathrm{E}+00$ & 7.99E-02 \\
\hline $45 \%$ & $6.24 \mathrm{E}-05$ & $2.97 \mathrm{E}-03$ & $7.00 \mathrm{E}-02$ & $1.62 \mathrm{E}-03$ & $2.23 \mathrm{E}-05$ & $1.19 \mathrm{E}-02$ & $0.00 \mathrm{E}+00$ & $0.00 \mathrm{E}+00$ & $0.00 \mathrm{E}+00$ & $0.00 \mathrm{E}+00$ & $0.00 \mathrm{E}+00$ & 8.80E-02 \\
\hline $50 \%$ & $6.55 \mathrm{E}-05$ & $3.20 \mathrm{E}-03$ & $7.83 \mathrm{E}-02$ & $1.67 \mathrm{E}-03$ & $2.37 \mathrm{E}-05$ & $1.26 \mathrm{E}-02$ & $0.00 \mathrm{E}+00$ & $0.00 \mathrm{E}+00$ & $0.00 \mathrm{E}+00$ & $0.00 \mathrm{E}+00$ & $0.00 \mathrm{E}+00$ & $9.69 \mathrm{E}-02$ \\
\hline $55 \%$ & $6.89 \mathrm{E}-05$ & $3.46 \mathrm{E}-03$ & $8.76 \mathrm{E}-02$ & $1.72 \mathrm{E}-03$ & $2.51 \mathrm{E}-05$ & $1.32 \mathrm{E}-02$ & $0.00 \mathrm{E}+00$ & $0.00 \mathrm{E}+00$ & $0.00 \mathrm{E}+00$ & $0.00 \mathrm{E}+00$ & $0.00 \mathrm{E}+00$ & $1.07 \mathrm{E}-01$ \\
\hline $60 \%$ & $7.26 \mathrm{E}-05$ & $3.75 \mathrm{E}-03$ & $9.92 \mathrm{E}-02$ & $1.77 \mathrm{E}-03$ & $2.66 \mathrm{E}-05$ & $1.39 \mathrm{E}-02$ & $0.00 \mathrm{E}+00$ & $0.00 \mathrm{E}+00$ & $0.00 \mathrm{E}+00$ & $0.00 \mathrm{E}+00$ & $0.00 \mathrm{E}+00$ & $1.18 \mathrm{E}-01$ \\
\hline $65 \%$ & 7.67E-05 & $4.06 \mathrm{E}-03$ & $1.11 \mathrm{E}-01$ & $1.84 \mathrm{E}-03$ & $2.85 \mathrm{E}-05$ & $1.47 \mathrm{E}-02$ & $0.00 \mathrm{E}+00$ & $0.00 \mathrm{E}+00$ & $0.00 \mathrm{E}+00$ & $0.00 \mathrm{E}+00$ & $0.00 \mathrm{E}+00$ & $1.30 \mathrm{E}-01$ \\
\hline $70 \%$ & $8.16 \mathrm{E}-05$ & 4.39E-03 & $1.26 \mathrm{E}-01$ & $1.91 \mathrm{E}-03$ & $3.05 \mathrm{E}-05$ & $1.55 \mathrm{E}-02$ & $0.00 \mathrm{E}+00$ & $0.00 \mathrm{E}+00$ & $0.00 \mathrm{E}+00$ & $0.00 \mathrm{E}+00$ & $0.00 \mathrm{E}+00$ & $1.44 \mathrm{E}-01$ \\
\hline $75 \%$ & $8.70 \mathrm{E}-05$ & $4.78 \mathrm{E}-03$ & $1.45 \mathrm{E}-01$ & $1.98 \mathrm{E}-03$ & $3.28 \mathrm{E}-05$ & $1.63 \mathrm{E}-02$ & $0.00 \mathrm{E}+00$ & $0.00 \mathrm{E}+00$ & $0.00 \mathrm{E}+00$ & $0.00 \mathrm{E}+00$ & $0.00 \mathrm{E}+00$ & $1.64 \mathrm{E}-01$ \\
\hline $80 \%$ & $9.35 \mathrm{E}-05$ & $5.26 \mathrm{E}-03$ & $1.69 \mathrm{E}-01$ & $2.06 \mathrm{E}-03$ & $3.57 \mathrm{E}-05$ & $1.73 \mathrm{E}-02$ & $0.00 \mathrm{E}+00$ & $0.00 \mathrm{E}+00$ & $0.00 \mathrm{E}+00$ & $0.00 \mathrm{E}+00$ & $0.00 \mathrm{E}+00$ & $1.88 \mathrm{E}-01$ \\
\hline $85 \%$ & $1.02 \mathrm{E}-04$ & $6.00 \mathrm{E}-03$ & $2.03 \mathrm{E}-01$ & $2.18 \mathrm{E}-03$ & $3.96 \mathrm{E}-05$ & $1.84 \mathrm{E}-02$ & $0.00 \mathrm{E}+00$ & $0.00 \mathrm{E}+00$ & $0.00 \mathrm{E}+00$ & $0.00 \mathrm{E}+00$ & $0.00 \mathrm{E}+00$ & $2.23 \mathrm{E}-01$ \\
\hline $90 \%$ & $1.14 \mathrm{E}-04$ & $6.97 \mathrm{E}-03$ & $2.51 \mathrm{E}-01$ & $2.35 \mathrm{E}-03$ & $4.52 \mathrm{E}-05$ & $1.97 \mathrm{E}-02$ & $0.00 \mathrm{E}+00$ & $0.00 \mathrm{E}+00$ & $0.00 \mathrm{E}+00$ & $0.00 \mathrm{E}+00$ & $0.00 \mathrm{E}+00$ & $2.70 \mathrm{E}-01$ \\
\hline $95 \%$ & $1.33 \mathrm{E}-04$ & 8.61E-03 & $3.52 \mathrm{E}-01$ & $2.59 \mathrm{E}-03$ & $5.44 \mathrm{E}-05$ & 2.13E-02 & $0.00 \mathrm{E}+00$ & $0.00 \mathrm{E}+00$ & $0.00 \mathrm{E}+00$ & $0.00 \mathrm{E}+00$ & $0.00 \mathrm{E}+00$ & $3.73 \mathrm{E}-01$ \\
\hline Mean & $7.23 \mathrm{E}-05$ & $3.79 \mathrm{E}-03$ & $1.19 \mathrm{E}-01$ & $1.74 \mathrm{E}-03$ & $2.69 \mathrm{E}-05$ & $1.26 \mathrm{E}-02$ & $6.47 \mathrm{E}-11$ & $1.72 \mathrm{E}-13$ & $2.49 \mathrm{E}-12$ & $2.39 \mathrm{E}-13$ & $3.84 \mathrm{E}-13$ & $1.37 \mathrm{E}-01$ \\
\hline Min & $7.59 \mathrm{E}-07$ & $9.27 \mathrm{E}-06$ & $2.85 \mathrm{E}-04$ & $1.19 \mathrm{E}-05$ & $4.10 \mathrm{E}-07$ & $1.46 \mathrm{E}-04$ & $0.00 \mathrm{E}+00$ & $0.00 \mathrm{E}+00$ & $0.00 \mathrm{E}+00$ & $0.00 \mathrm{E}+00$ & $0.00 \mathrm{E}+00$ & $4.63 \mathrm{E}-04$ \\
\hline Max & $1.91 \mathrm{E}-04$ & $2.87 \mathrm{E}-02$ & $2.15 \mathrm{E}+00$ & $8.69 \mathrm{E}-03$ & $1.91 \mathrm{E}-04$ & $2.57 \mathrm{E}-02$ & $1.61 \mathrm{E}-07$ & $3.34 \mathrm{E}-10$ & $6.19 \mathrm{E}-09$ & $5.22 \mathrm{E}-10$ & $5.42 \mathrm{E}-10$ & $2.18 \mathrm{E}+00$ \\
\hline
\end{tabular}




\section{TABLE C.31 Peak DSR Percentiles (mrem/yr per pCi/g) for Different Exposure Pathways for Sr-90 in Onsite Resident Scenario}

\begin{tabular}{|c|c|c|c|c|c|c|c|c|c|c|c|c|}
\hline Percentile & External & $\begin{array}{c}\text { Inhalation } \\
\text { w/o Rn }\end{array}$ & $\begin{array}{c}\text { Plant } \\
\text { Ingestion } \\
\text { (WI) }\end{array}$ & $\begin{array}{c}\text { Meat } \\
\text { Ingestion } \\
(\mathrm{WI})\end{array}$ & $\begin{array}{c}\text { Milk } \\
\text { Ingestion } \\
\text { (WI) }\end{array}$ & $\begin{array}{c}\text { Soil } \\
\text { Ingestion }\end{array}$ & $\begin{array}{c}\text { Water } \\
\text { Ingestion }\end{array}$ & $\begin{array}{l}\text { Aquatic } \\
\text { Foods }\end{array}$ & $\begin{array}{c}\text { Plant } \\
\text { Ingestion } \\
\text { (WD) }\end{array}$ & $\begin{array}{c}\text { Meat } \\
\text { Ingestion } \\
\text { (WD) }\end{array}$ & $\begin{array}{c}\text { Milk } \\
\text { Ingestion } \\
\text { (WD) }\end{array}$ & $\begin{array}{c}\text { All } \\
\text { Pathways }\end{array}$ \\
\hline $5 \%$ & $1.35 \mathrm{E}-05$ & $5.27 \mathrm{E}-09$ & $3.13 \mathrm{E}-03$ & 8.29E-04 & $1.96 \mathrm{E}-04$ & $1.79 \mathrm{E}-06$ & $0.00 \mathrm{E}+00$ & $0.00 \mathrm{E}+00$ & $0.00 \mathrm{E}+00$ & $0.00 \mathrm{E}+00$ & $0.00 \mathrm{E}+00$ & $4.42 \mathrm{E}-03$ \\
\hline $10 \%$ & $1.05 \mathrm{E}-04$ & $3.67 \mathrm{E}-08$ & $2.06 \mathrm{E}-02$ & $5.19 \mathrm{E}-03$ & $1.17 \mathrm{E}-03$ & $1.27 \mathrm{E}-05$ & $0.00 \mathrm{E}+00$ & $0.00 \mathrm{E}+00$ & $0.00 \mathrm{E}+00$ & $0.00 \mathrm{E}+00$ & $0.00 \mathrm{E}+00$ & $2.97 \mathrm{E}-02$ \\
\hline $15 \%$ & $2.37 \mathrm{E}-04$ & $7.46 \mathrm{E}-08$ & $3.82 \mathrm{E}-02$ & $9.20 \mathrm{E}-03$ & $2.26 \mathrm{E}-03$ & $2.57 \mathrm{E}-05$ & $0.00 \mathrm{E}+00$ & $0.00 \mathrm{E}+00$ & $0.00 \mathrm{E}+00$ & $0.00 \mathrm{E}+00$ & $0.00 \mathrm{E}+00$ & $5.30 \mathrm{E}-02$ \\
\hline $20 \%$ & $3.58 \mathrm{E}-04$ & $1.06 \mathrm{E}-07$ & 5.33E-02 & $1.34 \mathrm{E}-02$ & $3.15 \mathrm{E}-03$ & $3.85 \mathrm{E}-05$ & $0.00 \mathrm{E}+00$ & $0.00 \mathrm{E}+00$ & $0.00 \mathrm{E}+00$ & $0.00 \mathrm{E}+00$ & $0.00 \mathrm{E}+00$ & $7.31 \mathrm{E}-02$ \\
\hline $30 \%$ & $5.01 \mathrm{E}-04$ & $1.69 \mathrm{E}-07$ & $8.81 \mathrm{E}-02$ & $2.17 \mathrm{E}-02$ & $5.06 \mathrm{E}-03$ & $6.22 \mathrm{E}-05$ & $0.00 \mathrm{E}+00$ & $0.00 \mathrm{E}+00$ & $0.00 \mathrm{E}+00$ & $0.00 \mathrm{E}+00$ & $0.00 \mathrm{E}+00$ & $1.18 \mathrm{E}-01$ \\
\hline $35 \%$ & $5.53 \mathrm{E}-04$ & $1.91 \mathrm{E}-07$ & $1.06 \mathrm{E}-01$ & $2.65 \mathrm{E}-02$ & $6.18 \mathrm{E}-03$ & $7.26 \mathrm{E}-05$ & $0.00 \mathrm{E}+00$ & $0.00 \mathrm{E}+00$ & $0.00 \mathrm{E}+00$ & $0.00 \mathrm{E}+00$ & $0.00 \mathrm{E}+00$ & $1.44 \mathrm{E}-01$ \\
\hline $40 \%$ & $6.02 \mathrm{E}-04$ & $2.17 \mathrm{E}-07$ & $1.27 \mathrm{E}-01$ & $3.17 \mathrm{E}-02$ & $7.39 \mathrm{E}-03$ & 8.34E-05 & $0.00 \mathrm{E}+00$ & $0.00 \mathrm{E}+00$ & $0.00 \mathrm{E}+00$ & $0.00 \mathrm{E}+00$ & $0.00 \mathrm{E}+00$ & $1.70 \mathrm{E}-01$ \\
\hline $45 \%$ & $6.48 \mathrm{E}-04$ & $2.45 \mathrm{E}-07$ & $1.51 \mathrm{E}-01$ & $3.79 \mathrm{E}-02$ & $8.78 \mathrm{E}-03$ & $9.28 \mathrm{E}-05$ & $0.00 \mathrm{E}+00$ & $0.00 \mathrm{E}+00$ & $0.00 \mathrm{E}+00$ & $0.00 \mathrm{E}+00$ & $0.00 \mathrm{E}+00$ & $2.04 \mathrm{E}-01$ \\
\hline $50 \%$ & $6.92 \mathrm{E}-04$ & $2.77 \mathrm{E}-07$ & $1.74 \mathrm{E}-01$ & $4.39 \mathrm{E}-02$ & $1.04 \mathrm{E}-02$ & $1.02 \mathrm{E}-04$ & $0.00 \mathrm{E}+00$ & $0.00 \mathrm{E}+00$ & $0.00 \mathrm{E}+00$ & $0.00 \mathrm{E}+00$ & $0.00 \mathrm{E}+00$ & $2.35 \mathrm{E}-01$ \\
\hline $55 \%$ & $7.48 \mathrm{E}-04$ & $3.07 \mathrm{E}-07$ & $2.07 \mathrm{E}-01$ & $5.06 \mathrm{E}-02$ & $1.24 \mathrm{E}-02$ & $1.10 \mathrm{E}-04$ & $0.00 \mathrm{E}+00$ & $0.00 \mathrm{E}+00$ & $0.00 \mathrm{E}+00$ & $0.00 \mathrm{E}+00$ & $0.00 \mathrm{E}+00$ & $2.79 \mathrm{E}-01$ \\
\hline $60 \%$ & $7.99 \mathrm{E}-04$ & $3.40 \mathrm{E}-07$ & $2.37 \mathrm{E}-01$ & $5.92 \mathrm{E}-02$ & $1.46 \mathrm{E}-02$ & $1.21 \mathrm{E}-04$ & $0.00 \mathrm{E}+00$ & $0.00 \mathrm{E}+00$ & $0.00 \mathrm{E}+00$ & $0.00 \mathrm{E}+00$ & $0.00 \mathrm{E}+00$ & $3.21 \mathrm{E}-01$ \\
\hline $65 \%$ & $8.54 \mathrm{E}-04$ & $3.74 \mathrm{E}-07$ & $2.77 \mathrm{E}-01$ & $6.97 \mathrm{E}-02$ & $1.69 \mathrm{E}-02$ & $1.30 \mathrm{E}-04$ & $0.00 \mathrm{E}+00$ & $0.00 \mathrm{E}+00$ & $0.00 \mathrm{E}+00$ & $0.00 \mathrm{E}+00$ & $0.00 \mathrm{E}+00$ & $3.72 \mathrm{E}-01$ \\
\hline $80 \%$ & $1.08 \mathrm{E}-03$ & $5.15 \mathrm{E}-07$ & $4.55 \mathrm{E}-01$ & $1.19 \mathrm{E}-01$ & $2.92 \mathrm{E}-02$ & $1.61 \mathrm{E}-04$ & $0.00 \mathrm{E}+00$ & $0.00 \mathrm{E}+00$ & $0.00 \mathrm{E}+00$ & $0.00 \mathrm{E}+00$ & $0.00 \mathrm{E}+00$ & $6.07 \mathrm{E}-01$ \\
\hline $85 \%$ & $1.18 \mathrm{E}-03$ & $5.83 \mathrm{E}-07$ & $5.69 \mathrm{E}-01$ & $1.50 \mathrm{E}-01$ & $3.67 \mathrm{E}-02$ & $1.76 \mathrm{E}-04$ & $0.00 \mathrm{E}+00$ & $0.00 \mathrm{E}+00$ & $0.00 \mathrm{E}+00$ & $0.00 \mathrm{E}+00$ & $0.00 \mathrm{E}+00$ & $7.50 \mathrm{E}-01$ \\
\hline $90 \%$ & $1.33 \mathrm{E}-03$ & $6.92 \mathrm{E}-07$ & $7.29 \mathrm{E}-01$ & $1.94 \mathrm{E}-01$ & $4.76 \mathrm{E}-02$ & $1.93 \mathrm{E}-04$ & $0.00 \mathrm{E}+00$ & $0.00 \mathrm{E}+00$ & $0.00 \mathrm{E}+00$ & $0.00 \mathrm{E}+00$ & $0.00 \mathrm{E}+00$ & $9.71 \mathrm{E}-01$ \\
\hline $95 \%$ & $1.59 \mathrm{E}-03$ & $8.96 \mathrm{E}-07$ & $1.06 \mathrm{E}+00$ & $2.99 \mathrm{E}-01$ & $7.58 \mathrm{E}-02$ & $2.15 \mathrm{E}-04$ & $0.00 \mathrm{E}+00$ & $0.00 \mathrm{E}+00$ & $0.00 \mathrm{E}+00$ & $0.00 \mathrm{E}+00$ & $0.00 \mathrm{E}+00$ & $1.44 \mathrm{E}+00$ \\
\hline Mean & 7.37E-04 & $3.39 \mathrm{E}-07$ & $3.11 \mathrm{E}-01$ & $8.42 \mathrm{E}-02$ & $2.08 \mathrm{E}-02$ & $1.03 \mathrm{E}-04$ & $2.75 \mathrm{E}-04$ & $3.89 \mathrm{E}-06$ & $1.15 \mathrm{E}-05$ & $2.19 \mathrm{E}-05$ & $1.15 \mathrm{E}-05$ & $4.17 \mathrm{E}-01$ \\
\hline Min & $0.00 \mathrm{E}+00$ & $0.00 \mathrm{E}+00$ & $3.30 \mathrm{E}-29$ & $8.02 \mathrm{E}-30$ & $2.42 \mathrm{E}-30$ & $0.00 \mathrm{E}+00$ & $0.00 \mathrm{E}+00$ & $0.00 \mathrm{E}+00$ & $0.00 \mathrm{E}+00$ & $0.00 \mathrm{E}+00$ & $0.00 \mathrm{E}+00$ & $4.34 \mathrm{E}-29$ \\
\hline Max & $2.57 \mathrm{E}-03$ & $2.91 \mathrm{E}-06$ & $5.45 \mathrm{E}+00$ & $1.95 \mathrm{E}+00$ & $4.71 \mathrm{E}-01$ & $2.85 \mathrm{E}-04$ & $1.93 \mathrm{E}-01$ & $3.40 \mathrm{E}-03$ & $7.74 \mathrm{E}-03$ & $1.77 \mathrm{E}-02$ & $8.91 \mathrm{E}-03$ & $7.38 \mathrm{E}+00$ \\
\hline
\end{tabular}




\section{TABLE C.32 Peak DSR Percentiles (mrem/yr per pCi/g) for Different Exposure Pathways for Tc-99 in Onsite Resident Scenario}

\begin{tabular}{|c|c|c|c|c|c|c|c|c|c|c|c|c|}
\hline Percentile & External & $\begin{array}{c}\text { Inhalation } \\
\text { w/o Rn }\end{array}$ & $\begin{array}{c}\text { Plant } \\
\text { Ingestion } \\
(\mathrm{WI})\end{array}$ & $\begin{array}{c}\text { Meat } \\
\text { Ingestion } \\
\text { (WI) }\end{array}$ & $\begin{array}{c}\text { Milk } \\
\text { Ingestion } \\
(\mathrm{WI})\end{array}$ & $\begin{array}{c}\text { Soil } \\
\text { Ingestion }\end{array}$ & $\begin{array}{c}\text { Water } \\
\text { Ingestion }\end{array}$ & $\begin{array}{l}\text { Aquatic } \\
\text { Foods }\end{array}$ & $\begin{array}{c}\text { Plant } \\
\text { Ingestion } \\
\text { (WD) }\end{array}$ & $\begin{array}{c}\text { Meat } \\
\text { Ingestion } \\
\text { (WD) }\end{array}$ & $\begin{array}{c}\text { Milk } \\
\text { Ingestion } \\
\text { (WD) }\end{array}$ & $\begin{array}{c}\text { All } \\
\text { Pathways }\end{array}$ \\
\hline $5 \%$ & $6.58 \mathrm{E}-13$ & $7.02 \mathrm{E}-15$ & $2.00 \mathrm{E}-08$ & $4.44 \mathrm{E}-11$ & $5.21 \mathrm{E}-10$ & $6.19 \mathrm{E}-13$ & $0.00 \mathrm{E}+00$ & $0.00 \mathrm{E}+00$ & $0.00 \mathrm{E}+00$ & $0.00 \mathrm{E}+00$ & $0.00 \mathrm{E}+00$ & $1.60 \mathrm{E}-05$ \\
\hline $10 \%$ & $1.56 \mathrm{E}-12$ & $1.75 \mathrm{E}-14$ & 4.79E-08 & $1.12 \mathrm{E}-10$ & $1.26 \mathrm{E}-09$ & $1.56 \mathrm{E}-12$ & $0.00 \mathrm{E}+00$ & $0.00 \mathrm{E}+00$ & $0.00 \mathrm{E}+00$ & $0.00 \mathrm{E}+00$ & $0.00 \mathrm{E}+00$ & $4.02 \mathrm{E}-05$ \\
\hline $15 \%$ & $2.89 \mathrm{E}-12$ & $3.48 \mathrm{E}-14$ & $9.25 \mathrm{E}-08$ & $2.25 \mathrm{E}-10$ & $2.57 \mathrm{E}-09$ & $3.09 \mathrm{E}-12$ & $0.00 \mathrm{E}+00$ & $0.00 \mathrm{E}+00$ & $0.00 \mathrm{E}+00$ & $0.00 \mathrm{E}+00$ & $0.00 \mathrm{E}+00$ & $8.08 \mathrm{E}-05$ \\
\hline $20 \%$ & $5.07 \mathrm{E}-12$ & $5.84 \mathrm{E}-14$ & $1.58 \mathrm{E}-07$ & $3.97 \mathrm{E}-10$ & 4.45E-09 & $5.25 \mathrm{E}-12$ & $0.00 \mathrm{E}+00$ & $0.00 \mathrm{E}+00$ & $0.00 \mathrm{E}+00$ & $0.00 \mathrm{E}+00$ & $0.00 \mathrm{E}+00$ & $1.58 \mathrm{E}-04$ \\
\hline $25 \%$ & $7.82 \mathrm{E}-12$ & $9.38 \mathrm{E}-14$ & $2.69 \mathrm{E}-07$ & $6.72 \mathrm{E}-10$ & 7.29E-09 & $8.72 \mathrm{E}-12$ & $0.00 \mathrm{E}+00$ & $0.00 \mathrm{E}+00$ & $0.00 \mathrm{E}+00$ & $0.00 \mathrm{E}+00$ & $0.00 \mathrm{E}+00$ & $5.40 \mathrm{E}-04$ \\
\hline $30 \%$ & $1.26 \mathrm{E}-11$ & $1.47 \mathrm{E}-13$ & $4.40 \mathrm{E}-07$ & $1.10 \mathrm{E}-09$ & $1.26 \mathrm{E}-08$ & $1.30 \mathrm{E}-11$ & $1.41 \mathrm{E}-03$ & $1.85 \mathrm{E}-06$ & $1.27 \mathrm{E}-04$ & $8.90 \mathrm{E}-07$ & $2.34 \mathrm{E}-05$ & $1.68 \mathrm{E}-03$ \\
\hline $35 \%$ & $1.88 \mathrm{E}-11$ & $2.36 \mathrm{E}-13$ & 7.17E-07 & $1.71 \mathrm{E}-09$ & $2.06 \mathrm{E}-08$ & $2.02 \mathrm{E}-11$ & $2.91 \mathrm{E}-03$ & 5.19E-06 & 2.69E-04 & $2.00 \mathrm{E}-06$ & $5.30 \mathrm{E}-05$ & $3.40 \mathrm{E}-03$ \\
\hline $40 \%$ & $2.97 \mathrm{E}-11$ & $3.68 \mathrm{E}-13$ & $1.13 \mathrm{E}-06$ & $2.62 \mathrm{E}-09$ & $3.40 \mathrm{E}-08$ & $3.07 \mathrm{E}-11$ & $5.03 \mathrm{E}-03$ & $9.42 \mathrm{E}-06$ & 4.57E-04 & $3.57 \mathrm{E}-06$ & $8.96 \mathrm{E}-05$ & $5.71 \mathrm{E}-03$ \\
\hline $45 \%$ & $4.53 \mathrm{E}-11$ & $5.64 \mathrm{E}-13$ & $1.71 \mathrm{E}-06$ & 4.10E-09 & $5.20 \mathrm{E}-08$ & $4.63 \mathrm{E}-11$ & $7.51 \mathrm{E}-03$ & $1.48 \mathrm{E}-05$ & $7.23 \mathrm{E}-04$ & $5.83 \mathrm{E}-06$ & $1.41 \mathrm{E}-04$ & $8.61 \mathrm{E}-03$ \\
\hline $50 \%$ & $7.16 \mathrm{E}-11$ & 8.30E-13 & $2.66 \mathrm{E}-06$ & $6.57 \mathrm{E}-09$ & 7.82E-08 & $7.10 \mathrm{E}-11$ & $1.13 \mathrm{E}-02$ & 2.29E-05 & $1.11 \mathrm{E}-03$ & $9.07 \mathrm{E}-06$ & $2.11 \mathrm{E}-04$ & $1.29 \mathrm{E}-02$ \\
\hline $55 \%$ & $1.11 \mathrm{E}-10$ & $1.32 \mathrm{E}-12$ & 4.27E-06 & $1.07 \mathrm{E}-08$ & $1.26 \mathrm{E}-07$ & $1.13 \mathrm{E}-10$ & $1.70 \mathrm{E}-02$ & $3.39 \mathrm{E}-05$ & $1.59 \mathrm{E}-03$ & $1.32 \mathrm{E}-05$ & $3.23 \mathrm{E}-04$ & $1.95 \mathrm{E}-02$ \\
\hline $60 \%$ & $1.70 \mathrm{E}-10$ & $2.01 \mathrm{E}-12$ & $6.58 \mathrm{E}-06$ & $1.75 \mathrm{E}-08$ & $2.02 \mathrm{E}-07$ & $1.71 \mathrm{E}-10$ & $2.43 \mathrm{E}-02$ & $5.10 \mathrm{E}-05$ & $2.33 \mathrm{E}-03$ & $1.86 \mathrm{E}-05$ & $4.81 \mathrm{E}-04$ & $2.79 \mathrm{E}-02$ \\
\hline $65 \%$ & $2.69 \mathrm{E}-10$ & $3.34 \mathrm{E}-12$ & $1.04 \mathrm{E}-05$ & $2.68 \mathrm{E}-08$ & $3.16 \mathrm{E}-07$ & $2.71 \mathrm{E}-10$ & $3.52 \mathrm{E}-02$ & 7.30E-05 & $3.34 \mathrm{E}-03$ & $2.56 \mathrm{E}-05$ & 7.03E-04 & $3.98 \mathrm{E}-02$ \\
\hline $70 \%$ & $4.23 \mathrm{E}-10$ & $5.28 \mathrm{E}-12$ & $1.64 \mathrm{E}-05$ & $4.20 \mathrm{E}-08$ & $5.09 \mathrm{E}-07$ & 4.32E-10 & 4.95E-02 & $1.04 \mathrm{E}-04$ & $4.72 \mathrm{E}-03$ & $3.60 \mathrm{E}-05$ & $9.62 \mathrm{E}-04$ & $5.62 \mathrm{E}-02$ \\
\hline $75 \%$ & $6.36 \mathrm{E}-10$ & $8.24 \mathrm{E}-12$ & $2.55 \mathrm{E}-05$ & $6.56 \mathrm{E}-08$ & 7.92E-07 & $7.00 \mathrm{E}-10$ & $6.82 \mathrm{E}-02$ & $1.57 \mathrm{E}-04$ & $6.45 \mathrm{E}-03$ & $5.07 \mathrm{E}-05$ & $1.33 \mathrm{E}-03$ & $7.78 \mathrm{E}-02$ \\
\hline $80 \%$ & $1.00 \mathrm{E}-09$ & $1.24 \mathrm{E}-11$ & $4.15 \mathrm{E}-05$ & $1.10 \mathrm{E}-07$ & $1.29 \mathrm{E}-06$ & $1.08 \mathrm{E}-09$ & 8.97E-02 & $2.24 \mathrm{E}-04$ & $8.70 \mathrm{E}-03$ & $6.87 \mathrm{E}-05$ & $1.82 \mathrm{E}-03$ & $1.03 \mathrm{E}-01$ \\
\hline $85 \%$ & $1.58 \mathrm{E}-09$ & $1.97 \mathrm{E}-11$ & $6.62 \mathrm{E}-05$ & $1.78 \mathrm{E}-07$ & $2.17 \mathrm{E}-06$ & $1.71 \mathrm{E}-09$ & $1.20 \mathrm{E}-01$ & $3.31 \mathrm{E}-04$ & $1.15 \mathrm{E}-02$ & $9.34 \mathrm{E}-05$ & $2.52 \mathrm{E}-03$ & $1.36 \mathrm{E}-01$ \\
\hline $90 \%$ & $2.77 \mathrm{E}-09$ & $3.49 \mathrm{E}-11$ & $1.16 \mathrm{E}-04$ & $3.40 \mathrm{E}-07$ & $3.80 \mathrm{E}-06$ & 2.95E-09 & $1.61 \mathrm{E}-01$ & $5.07 \mathrm{E}-04$ & $1.55 \mathrm{E}-02$ & $1.36 \mathrm{E}-04$ & $3.72 \mathrm{E}-03$ & $1.83 \mathrm{E}-01$ \\
\hline $95 \%$ & $5.48 \mathrm{E}-09$ & $7.28 \mathrm{E}-11$ & $2.89 \mathrm{E}-04$ & $8.67 \mathrm{E}-07$ & $1.01 \mathrm{E}-05$ & $6.14 \mathrm{E}-09$ & $2.25 \mathrm{E}-01$ & $9.41 \mathrm{E}-04$ & $2.35 \mathrm{E}-02$ & $2.22 \mathrm{E}-04$ & $6.02 \mathrm{E}-03$ & $2.54 \mathrm{E}-01$ \\
\hline Mean & $5.13 \mathrm{E}-07$ & 7.46E-09 & $3.53 \mathrm{E}-03$ & $1.11 \mathrm{E}-05$ & $1.20 \mathrm{E}-04$ & $5.76 \mathrm{E}-07$ & 4.98E-02 & $2.08 \mathrm{E}-04$ & 5.39E-03 & 4.77E-05 & $1.25 \mathrm{E}-03$ & $5.68 \mathrm{E}-02$ \\
\hline Min & $1.08 \mathrm{E}-14$ & $1.67 \mathrm{E}-16$ & $3.36 \mathrm{E}-10$ & $6.82 \mathrm{E}-13$ & $1.34 \mathrm{E}-11$ & $5.25 \mathrm{E}-15$ & $0.00 \mathrm{E}+00$ & $0.00 \mathrm{E}+00$ & $0.00 \mathrm{E}+00$ & $0.00 \mathrm{E}+00$ & $0.00 \mathrm{E}+00$ & $2.07 \mathrm{E}-07$ \\
\hline Max & $5.64 \mathrm{E}-05$ & $1.82 \mathrm{E}-06$ & 4.67E-01 & $3.90 \mathrm{E}-03$ & $2.44 \mathrm{E}-02$ & $5.69 \mathrm{E}-05$ & $5.53 \mathrm{E}-01$ & $1.38 \mathrm{E}-02$ & $1.60 \mathrm{E}-01$ & $1.58 \mathrm{E}-03$ & $3.84 \mathrm{E}-02$ & $6.38 \mathrm{E}-01$ \\
\hline
\end{tabular}





\section{Argonne}

\section{Environmental Science Division}

Argonne National Laboratory

9700 South Cass Avenue, Bldg. 240

Argonne, IL 60439-4847

www.anl.gov

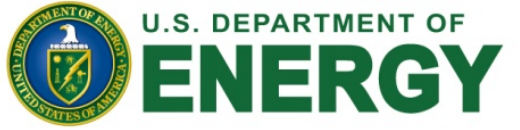

Argonne National Laboratory is a U.S. Department of Energy laboratory managed by UChicago Argonne, LLC 